\title{
"A Language of Many Hats": The Rise of Sheng and other Linguistic Styles among Urban Youth in Kenya
}

\author{
Michael Gathonjia Wairungu \\ Charlottesville, Virginia
}

M.A. Anthropology, University of Virginia, 2010

B.Ed. Swahili and Geography, Moi University, 2004

A Dissertation presented to the Graduate Faculty of the University of Virginia in Candidacy for the Degree of Doctor of Philosophy

\author{
Department of Anthropology \\ University of Virginia \\ May 2014
}

Dr. Ellen Contini-Morava (Chair)

Dr. Adria LaViolette

Dr. Daniel Lefkowitz

Dr. George Mentore

Dr. Diane Hoffman (Outside Reader) 


\begin{abstract}
In Kenya today, there is a disconnect between the national language policy and peoples' language use in actual practice. While the government promotes Standard Swahili as a national language to facilitate interethnic communication and alongside English for use in official functions, my research findings indicate that many young Kenyans do not adhere to the said national language policy. Instead, they have embraced unauthorized nonstandard language varieties such as Sheng for distinctiveness among other reasons. I argue that these young Kenyans find the other readily available languages insufficient for their communicative needs, and for negotiating desired distinct social identities. However, while embracing these alternative linguistic forms, speakers engage in social practices that suggest both resistance to and conformity with the official language ideology and local cultural frameworks.
\end{abstract}

The research study was inspired by three common claims about Sheng which had not been researched before: 1) that use of Sheng among students interferes with their mastery of the officially sanctioned Standard Swahili and English; 2) Sheng unites speakers from diverse ethnic backgrounds; and 3) Sheng has spread beyond its perceived origins, Nairobi. In order to investigate these claims, I conducted an intensive ethnographic study in two co-ed schools in Nakuru and Mombasa, two major towns in Kenya with underlying regional and ethnic tensions. Mombasa provided an additional context for investigating how Sheng is used and perceived alongside competing varieties of Swahili. My study therefore introduces a new point 
of departure in the study of Sheng by focusing on social and geographical contexts that had not been explored before.

My research found that previous scholarly attempts to define Sheng are inadequate because they did not account for social factors such as speakers' attitudes, which influence how they perceive and employ Sheng. This finding also challenges the common association of Sheng with urban youth since not all youth speak Sheng, and not all youth language varieties are called Sheng. Instead, what speakers call Sheng is socially defined. I therefore propose that Sheng is best understood via Irvine's (2001) concept of style, i.e. "social semiosis of distinctiveness."

Additional findings address the relationship between language and national identity, and also question the viability of the notions of "standard language ideology" (Lippi-Green 2012) and "legitimate language" (Bourdieu 1977). The two notions do not fit well in Kenya, especially in the case of Standard Swahili. Though Standard Swahili is both a national and official language, it is not associated with prestige and power. Instead, it is English, the former colonial language, which generates social rewards among adults, and nonlinguistic varieties such as Sheng among young Kenyans. Overall, my dissertation will contribute to the study of Sheng and other urban youth language varieties in Kenya, and the rest of multilingual post-colonial Africa. Also, my findings could inform language planning and formulation of educational policies in these multilingual settings. 


\section{Dedication}

To my parents, Wairungu wa Murebi and Wanjiru wa Wairungu. 


\section{Acknowledgments}

This dissertation project is a culmination of many years of hard work.

However, I would never have been able to walk the long academic journey without the guidance of my committee members, help from friends, and support from my family.

I would like to thank my academic mentor and chair of my dissertation committee, Professor Ellen Contini-Morava, for her excellent guidance and creating a conducive learning atmosphere throughout the period I have been at the University of Virginia. I would also like to appreciate other committee members for their support and guidance: Professors Adria LaViolette, Daniel Lefkowitz, George Mentore and Diane Hoffman. Your patience, care and kindness have brought me this far. Besides my committee members, I was lucky to have professors who offered magnificent moral support. Thanks to Susan Mackinon, Ira Bashkow, Sasha Newell, Peter Metcalf, Eve Danziger, Fred Damon, Lise Dobrin and Wende Marshall. Outside the Department of Anthropology, I would like to thank Jack Morava, Deborah McDowell, Cynthia Hoehler-Fatton, Carol Anne Spreen, Salim Valley, Sangai Mohochi, James and Miriam Ngundi, Besi Muhonja, Gitile Naituli, Njoki Osotsi, Justus Mbae, Nathaniel Ogechi, Shuaib Lwasa, and Gatua Wa'Mbugwa. You are great sources of inspiration to me.

To my friends within the University of Virginia; thanks to Ann Githinji, Lydia Rodriguez, Sergio Lopez, Chrissie Monaghan, Pamela Kimeto, Erin Nourse, Jack Stoetzel, Abby Holeman, Yuchien Huang, and all other graduate students at the Department of Anthropology. Outside the University of Virginia, I would like to 
appreciate Anne Karanja, Jean Siler, Marigail Wynne, Margaret Gacheru, Eddah Waitara, Anthony Nyakweya, Elvis Sing'oei, Alexina Karanja, Sharon Muita and Paul Ndolo. Also, the families of John Wachori, Shadrack Nthusi and Francis Agola. So many friends helped me in many ways that it is impossible to thank everyone individually. You are all part of my success.

Special thanks to teachers, students and other members of staff at Someni and Vikapuni high schools. It is through your cooperation that I was able to conduct my research. Finally, I would like to appreciate the endless love and support from my immediate family members, the Wairungus. I love you all. 


\section{Contents}

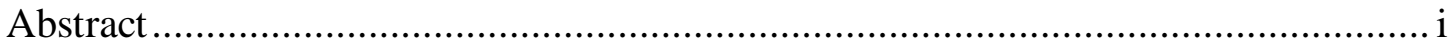

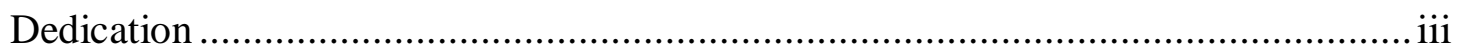

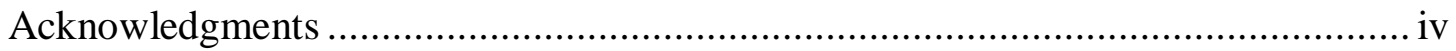

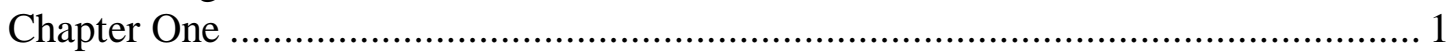

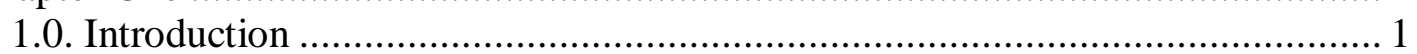

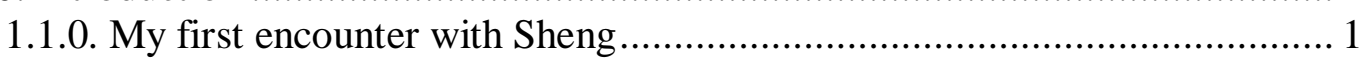

1.2.0. Language policy vs. language use in Kenya ......................................... 3

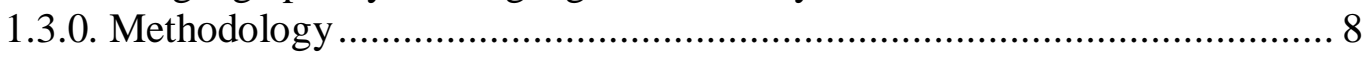

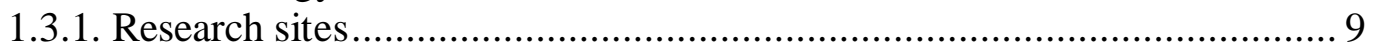

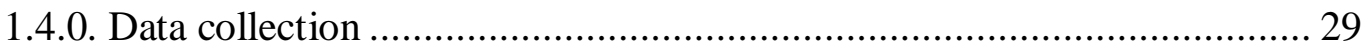

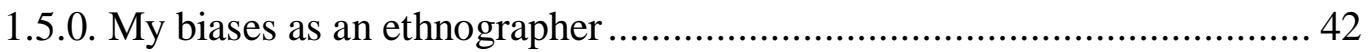

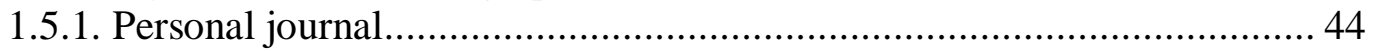

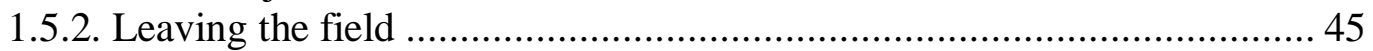

1.5.3. Follow-up interviews via Facebook and E-mail ................................... 45

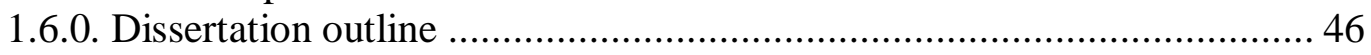

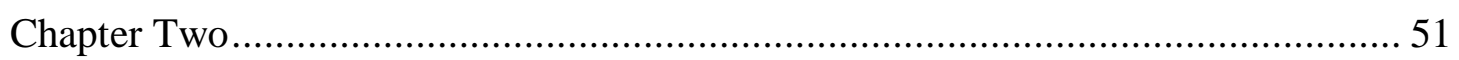

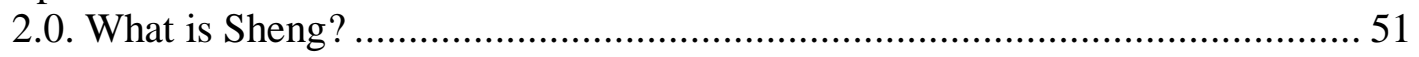

2.1.0. Previous scholarly definitions of Sheng ........................................... 53

2.2.0. Sheng vs. Nairobi Swahili: how different are they? ............................ 55

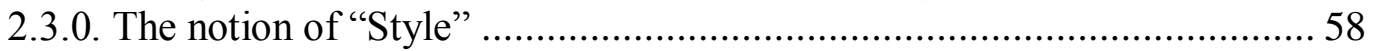

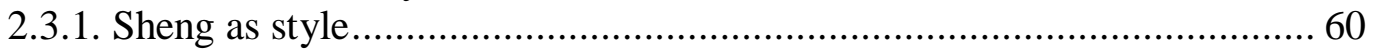

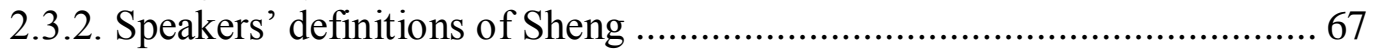

2.4.0. Sheng and other urban youth vernaculars in post-colonial Africa............ 88

2.4.1. Main social uses of urban youth vernaculars......................................... 90

2.5.0. Attitudes expressed toward African urban youth vernaculars ................. 94

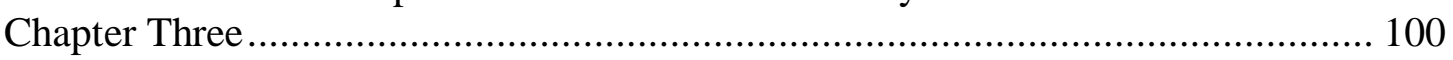

3.0. The National Language Policy in Kenya: Theory vs. Practice .................... 100

3.1.0. Kenya's formal education and associated language policy during the

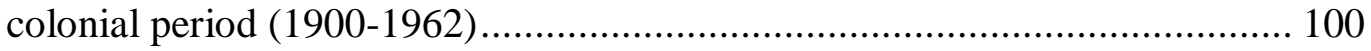

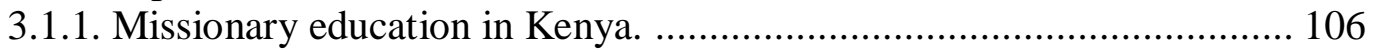

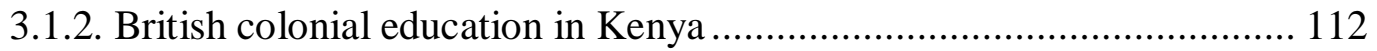

3.2.0. Language policy during the colonial period ..................................... 115

3.2.1. Colonial language policy between 1900-1929.................................. 115

3.2.2. Colonial language policy between $1930-1949$..................................... 121

3.2.3. Colonial language policy between 1950-1962 .................................... 126

3.3.0. Kenya's language policy from 1963 to date .................................... 130

3.3.1. Educational commissions of inquiry from 1963 to the present ............. 131

3.3.2. "Roadside" or impromptu declarations by politicians ........................ 137

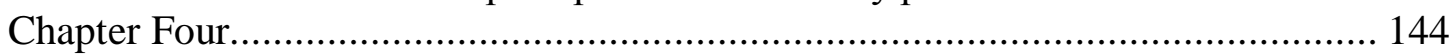

4.0. Sheng, Ethnicity and Politics of National Identity in Kenya ...................... 144

4.1.0. Perceived "ethnic neutrality" of Standard Swahili as a national language

4.2.1. A comparison of Standard Swahili and Kimvita ................................ 154

4.2.2. The rivalry between Kimvita and Standard Swahili ........................... 156 
4.3.0. English versus Standard Swahili in Kenya.

4.4.0. Comparison between the status of Standard Swahili as a national and official language in Kenya and neighboring Tanzania

4.5.0. Standard Swahili versus urban youth vernaculars such as Sheng: theory versus practice in the national language policy ......................................... 184

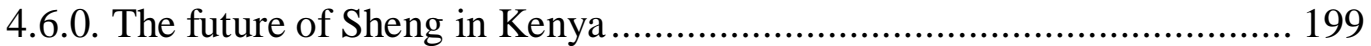

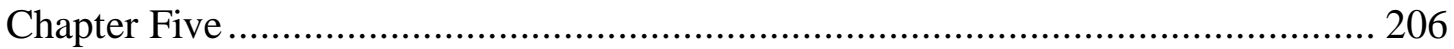

5.0. Nonstandard Language Varieties, Education and the Paradoxical Official

Language Ideology in Kenya ........................................................................... 206

5.1.0. "Standard language ideology" in Kenya and associated identities ......... 208

5.1.1. "Legitimate language" and the politics of linguistic "correctness" in Kenya

5.1.2. "Inequality between two equals": English vs. Standard Swahili in Kenya 215

5.1.3. Language vs. Body hexis: the dialectics of uniformity in Kenyan schools

226

5.1.4. Does fighting the "usual suspects" eradicate them really? The question of nonstandard language varieties in schools ................................................... 235

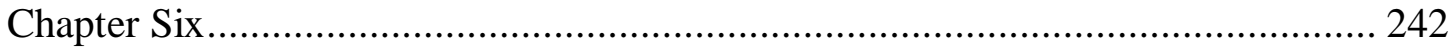

6.0. Style, Fashion and Identity: Sheng and lugha ya mtaa ............................. 242

6.1.0. Sheng and lugha ya mtaa: styling to rebel ..................................... 243

6.1.1. Speakers' language choices and associated attitudes.......................... 248

6.1.2. Sheng and academic performance: is it a resource or an impediment?... 252

6.1.3. Use of Sheng to criticize and mock authority .................................... 255

6.1.4. "Streets" and "Ghetto" as sites of ingeniousness ................................. 256

6.1.5. Nonstandard vs. standard grammar: stretching the limits .................... 261

6.1.6. Formality vs. informality among speakers of Sheng and lugha ya mtaa 262

6.1.7. Non-linguistic dimensions of Sheng and lugha ya mtaa....................... 279

6.2.0. Speakers' compliance to the "Standard Language Ideology" ............... 282

6.3.0. The role of gender in speakers' use of Sheng and lugha ya mtaa .......... 286

Chapter Seven ............................................................................................... 305

7.0. "Bringing Them Down": "Shrubbing” Discourse among Young Kenyans ... 305

7.1.0. The notion of shrubbing vs. Mother tongue interference: a question of

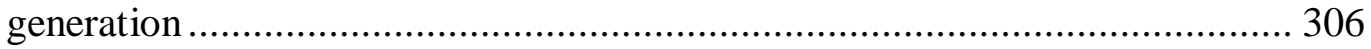

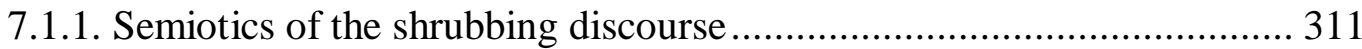

7.1.2. Conformity in the shrubbing discourse ............................................... 318

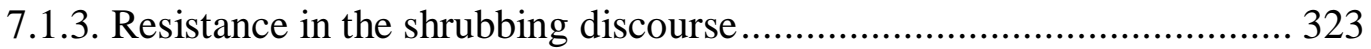

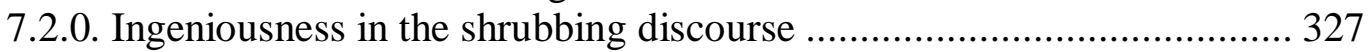

7.2.1. The shrubbing discourse in Nakuru ................................................. 327

7.2.2. The notion of shrubbing among speakers of lugha ya mtaa in Mombasa

7.3.0. Shrubbing as symbolic capital

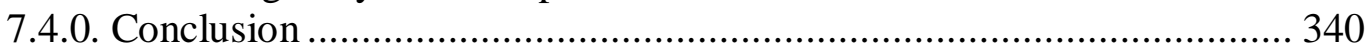

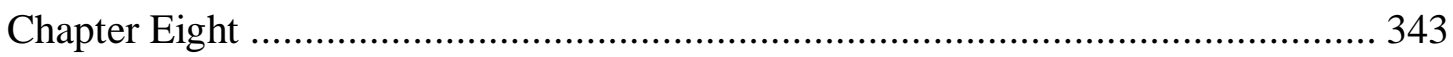

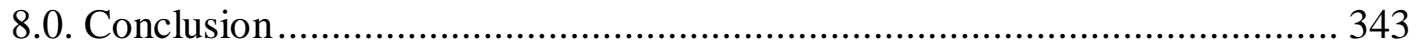

8.1.0. Summary of the main arguments and conclusions............................. 343

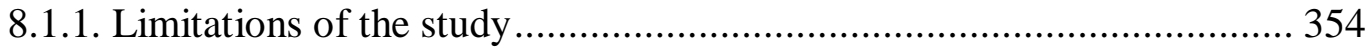




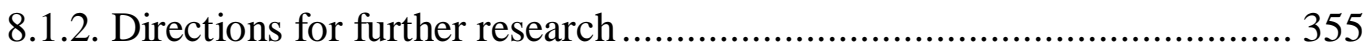

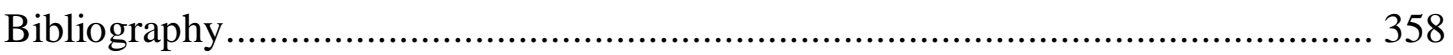

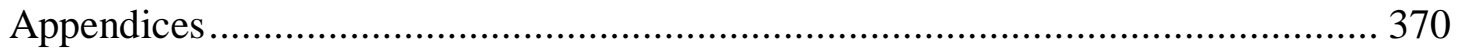

Appendix A: Sample print material used for eliciting comments ..................... 370

Appendix B: Sample print material used for eliciting comments. ..................... 371

Appendix C: Modified school uniform to negotiate swag ............................... 371

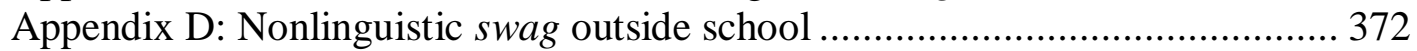

Appendix E: The revised Vikapuni School language policy ............................ 373

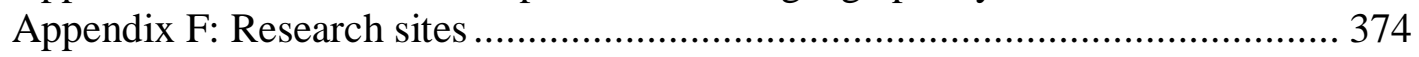




\section{Chapter One}

\subsection{Introduction}

\subsubsection{My first encounter with Sheng}

When I entered high school in 1995, I met and socialized with fellow students from both rural and urban areas. My high school was located in a rural setting in central Kenya. Peers from major cities such as Nairobi, Nakuru and Kisumu spoke a language variety that really fascinated me. It sounded familiar and different at the same time: speakers used English and Swahili words but there were times, when referring to common items such as shoes, tea or food, they would introduce vocabulary that I had not heard before. These speakers also rarely referred to people and places using their full names. For example, if they were talking about their residential neighborhoods such as Umoja, Kawangware or Kaloleni in Nairobi, they would say Umoo, Ungwaroo and Ololoo respectively. Such linguistic modifications and their urban accent made their way of speaking attractive to me, creating a strong desire to learn it.

However, these speakers would talk about Nairobi city and "street" life in an exaggerated manner, and engaged in issues which made me fear ever visiting Nairobi. Specifically, they bluffed about their mastery of the city and how they engaged in criminal activities such as confronting or dodging police in the streets, or how they would con their parents of money "to go out for fun." They would also laugh at us rural peers whenever we tried speaking like them because we spoke English and Swahili with a mother tongue accent. I loved the language variety but feared its speakers. 
Interestingly, some of these speakers came to our school because their rural roots were in the immediate neighborhoods, and their parents wanted to remove them from the corrupting influence of the city, but they rarely identified with these roots. Instead, they defined themselves as majamaa wa Nai, 'guys from Nairobi', while referring to rural peers like me as majamaa wa shagz or ushagoo, 'country guys'. While such aspects of differentiation discouraged me from associating with these urban peers, there were some rural peers who admired the "street" life of these speakers and made efforts to learn their way of speaking, eventually embracing their street-wise identity. One such peer who was my friend told me that the language variety in question was called Sheng and was spoken in Nairobi.

I also encountered Sheng when I entered Moi University in Eldoret among colleagues from different parts of the country many of whom claimed were from Nairobi. The question that lingered in my mind was why most speakers of Sheng that I met associated themselves with Nairobi even when I knew they were not from there. At the time, I did not attach a scholarly concern to Sheng because I had already labeled it a "language of bad people." Also, I was trained as a teacher of Standard Swahili, and I had absorbed the negative attitudes toward Sheng that were common among teachers of Standard Swahili. However, my negative perception changed after I entered the University of Virginia where I was introduced to literatures focusing on language varieties such as Sheng. I became aware of the ongoing debate among scholars of Sheng about its origin and social uses. Most significantly, I gathered anecdotal evidence that though many Kenyans associated Sheng with Nairobi, it had spread to other major towns and rural areas as well (Abdul Aziz and 
Osinde 1997; Bosire 2008; Githiora 2002; Spyropoulos 1987). I became interested in finding out to what extent this was true.

Despite this hope of finding Sheng outside Nairobi, the friends I met when I went to conduct my fieldwork in 2011 kept steering me toward Nairobi, arguing that I would not find Sheng elsewhere. It was partly the controversy surrounding the existence of Sheng beyond Nairobi, and whether it was called Sheng in these other settings, that inspired this research. The study was also inspired by the common claim among educators and other figures of authority, that students' use of Sheng interferes with their mastery of the officially sanctioned Standard Swahili and English, which further leads to their poor performance in national exams (Momanyi 2009; Standard Newspaper, December $29^{\text {th }} 2011$ ). In order to address these concerns, my main research questions were, first, whether Sheng has indeed spread beyond Nairobi, and if so how? Second, in settings where Sheng is used, how is it used and perceived in relation to the officially sanctioned Standard Swahili and English and other languages spoken in high schools in Kenya? (I will discuss the choice of my research settings towards the end of the section below.)

\subsubsection{Language policy vs. language use in Kenya}

Kenya is a multilingual country with approximately 42 officially recognized “ethnic languages," in addition to Standard Swahili and English. ${ }^{1}$ However, as mentioned earlier, there is a stark disconnect between the official/national language

\footnotetext{
${ }^{1}$ In the Kenyan context, the term "ethnic languages" is used to refer to the languages associated with the approximately 42 named ethnic groups in Kenya. For example, Kikuyu language for Kikuyu ethnic community, Dholuo for Luo ethnic community (Samper 2002; The National Constitution 2010). However, the notion of "ethnic language" is problematic colonial construct introduced by the early European missionaries for the purposes of codifying African languages to be used in spreading Christianity (Irvine and Gal 1995).
} 
policy and peoples' actual language use. According to the National Constitution (2010), the government recognizes Standard Swahili as the national language in order to facilitate inter-ethnic communication, hence to promote national unity (see Nabea 2009). Both Standard Swahili and English are the official languages and are supposed to be used in conducting formal government transactions such as court proceedings, parliamentary debates and educational instruction. The Constitution is not explicit about the role of the "ethnic languages" even though it acknowledges the will of the government to promote and develop them. Specifically, the National Constitution (2010: 14) states that, "[t]he State shall promote and protect the diversity of language of the people of Kenya... and promote the development and use of indigenous languages...."

However, Kenya's national language policy mainly exists in theory, as both government officials and ordinary Kenyans often use the various language varieties in undesignated domains, causing linguistic overlaps and mismatches (Mugane 2005). For example, although Standard Swahili is the national language and one of the official languages, the 2013 presidential and vice-presidential debates were conducted in English, despite ostensibly being aimed at the general population, many of whom have limited knowledge of English. ${ }^{2}$ Many Kenyans do not use the officially designated languages in the ways prescribed by the government. For example, Standard Swahili is rarely used except when taught in the classroom as a subject and some media functions such as news reporting. Police officers are known to use a distinct variety of Swahili that is not standard. Further, many Kenyans use more than

\footnotetext{
${ }^{2}$ The full video of the presidential debate can be accessed from this site: http://www.youtube.com/user/standardgroupkenya?feature=watch
} 
one language variety in a single conversation, making language switching and mixing the norm in Kenya, especially in urban areas (Myers-Scotton 1993).

Similar language practices that contravene the official language policy have been reported in schools. Official education policy on language states that the medium of classroom instruction in elementary schools should be the dominant "ethnic language" in ethnically homogenous areas and Standard Swahili in the cosmopolitan areas for the first three years of education (Bunyi 2005; Eshiwani 1993). Elementary schools are then supposed to switch to English-medium instruction from grade four onwards in all other subjects apart from Swahili. However, Bunyi (2005) reports that it is common for teachers in elementary schools in Kenya to use more than one language in the same lesson. Similarly, my field observations indicate that both teachers and students in high schools do not adhere to the schools' language policy all the time. Instead, they employ language practices that most informants claimed were neither Standard Swahili nor English.

My study found that many students prefer speaking non-sanctioned varieties such as Sheng because they index linguistic ingeniousness among peers. They also find nonstandard varieties more appropriate for their communicative needs and negotiating desired distinct social identities than the other readily available language varieties. I argue that use of nonstandard varieties among peers generates more social rewards than the officially sanctioned Standard Swahili and English. In Nakuru, for example, I asked students in one of the mixed-gender focus groups about their understanding of Sheng and how peers regarded a student who did not know it. They reported that such students were often under intense pressure to learn Sheng or they 
will always be quiet. One of them, Kadogo, ${ }^{3}$ whose family lives in the rural areas but lives with her relatives in Nakuru town, shared her personal experience about how she went through a hard time transitioning from "country" to urban life because she did not know Sheng. Also, she feared speaking the Swahili she knew because she had mother-tongue influence. Speaking Swahili or English with the influence of ethnic language is stigmatized among young Kenyans, especially in urban areas (See chapter seven for detail). In the long run, Kadogo reported that she had to learn Sheng with the help of her friend in order to fit in (Praise and Worship focus group, 11/2011-Nakuru).

While Kadogo's experience demonstrates that knowledge of Sheng is highly regarded among young Kenyans, various figures of authority such as teachers and parents attribute students' poor performance in Standard Swahili and English to their use of Sheng. Schools have therefore intensified the fight against Sheng, claiming that its use among students should be abolished. However, since use of Sheng generates more social rewards among peers than the officially sanctioned Standard Swahili and English, the more schools fight Sheng, the more it flourishes. Some scholars such as Migunda-Attyang (2007:21) claim that, "Sheng is gradually usurping the place of Kiswahili as a lingua franca in Nairobi," but I seek to challenge such assumptions because Standard Swahili has never been a lingua franca for everyday communication in actual practice. Instead, it is contact varieties of Swahili among adults and emergent urban vernaculars such as Sheng among many young Kenyans, which have historically played that role (see Chapter Four).

\footnotetext{
${ }^{3}$ All names of informants and schools used in this dissertation are pseudonyms for purposes of confidentiality. Also, please note that I do not state my informants' ethnicity, especially students because many of them were from mixed ethnic backgrounds and this made it hard to establish their ethnicity during fieldwork.
} 
Since previous research on Sheng was limited to Nairobi, I sought to focus on other major urban areas, specifically Nakuru and Mombasa, to find out how Sheng is used and perceived in relation to Standard Swahili, English and other languages spoken in contexts where countervailing factors may work against the common identity that Sheng promises. I chose these two towns because, despite the anecdotal evidence that Sheng had spread to various major urban areas in Kenya, no in-depth study of how Sheng is used and perceived in its new settings had been done prior to mine. Specifically, Nakuru and Mombasa were appropriate for the present research because they had experienced periodic ethnic conflict surrounding general elections. Additionally, Mombasa provided a context where Sheng competes with Standard Swahili and a local coastal variety of Swahili called Kimvita, which is highly regarded by its speakers. Therefore, my research sought to find out how Sheng, which arose in Nairobi as a lingua franca among many young Kenyans from diverse ethnic backgrounds (Abdul Aziz and Osinde 1997; Spyropoulos 1987), responds to ethnic and regional polarization on the one hand, and how it is used and evaluated in an environment where it competes with both local and "standard" ${ }^{4}$ varieties of Swahili on the other.

I focused on schools because of the association of Sheng with youth, and because schools are settings where Sheng is highly discouraged. Also, despite numerous claims by various figures of authority such as educators and parents that students' use of Sheng interferes with their mastery of the officially sanctioned Standard Swahili and English, there has been no prior research based on direct observation of how Sheng is used and perceived in schools. My research therefore

\footnotetext{
${ }^{4}$ Note that speakers of the Kimvita variety of Swahili contest that Swahili should be standardized based on Kimvita (see Chapter Four).
} 
sought to know whether Sheng is spoken in schools, who speaks it, when and why they do it.

My research was timely given that the current Minister of Education recently called for in-depth research to establish what ails language teaching in Kenya, rather than relying on the unsubstantiated claim that Sheng is the cause. This call was echoed by renowned newspaper columnist Philip Ochieng, who argued in the Daily Nation Newspaper (September $21^{\text {st }} 2013$ ) that:

Every time our children perform poorly in language examinations, every 'intellectual' readily names Sheng as the demon. No, I will not deny this allegation because even I have not studied the problem scientifically. But that is precisely why I cannot just accept the claim either. I will take it seriously only when I have read a cogent public statement detailing the conditions in which the findings were reached. Is Sheng the culprit in the plummeting standard of English in this country? Why is it impossible to know both Sheng and good English simultaneously? Since my mind is always open to new knowledge, I promise to swallow your statement as soon as I have seen the facts and figures - as soon as you convince me that Sheng is intrinsically so powerful that, in its presence, even a language superpower like English must cower and give way... Do not, then, fob off your educational failings to our youth in their linguistic inventiveness. Say NO to what are but useless commonsensical opinions on Sheng. Instead, prevail on Prof Jacob Kaimenyi [the Minister of Education] to discover the truth by conducting a proper inquiry into what is ailing language teaching in Kenya.

Given that my research coincides with the Minister's call, my findings have the potential to inform policy decisions on language and education in Kenya (I discuss these findings in detail later in the dissertation.) Next, I discuss the two research sites and the various methods I employed to collect the data necessary to answer my research questions.

\subsubsection{Methodology}

I collected the data used in this dissertation in two phases. The first phase was an intensive ethnographic study for ten months in two ethno-linguistically diverse co-ed high schools, one in Nakuru and the other in Mombasa. The second phase involved 
post-fieldwork follow-up interviews with a few teachers and students, via facebook and e-mail, for a period of seven months.

I arrived in Kenya from the U.S. on August $12^{\text {th }} 2011$. I was housed temporarily by one of my high school friends called Shemeji who lives and works in Nairobi. I sought to reorient myself to life in Kenya by visiting my immediate family members and close friends since the country had changed significantly since my previous visit in 2008. I also spent this time gathering basic information about the security situation in Nakuru and Mombasa. This was a major concern because of my ethnicity as Kikuyu, since the research coincided with a time when Kenya was preparing for a general election which was as ethnically charged as the previous one. During that election in 2007, the Kikuyu was one of the targeted ethnic groups in the violence that ensued. Therefore, it was necessary to have some reassurance that I would be safe doing my research in the two research sites. By the end of the second week, I had gathered the necessary information and was ready to start my fieldwork. I applied and received authorization to conduct research in both Nakuru and Mombasa from the government headquarters in Nairobi, then headed to Nakuru, my first research site. In the remainder of this chapter, I describe the two research sites, my field experience and data collection methods.

\subsubsection{Research sites}

\subsubsection{Nakuru}

Nakuru is the fourth largest urban center in Kenya after Nairobi, Kisumu and Mombasa. It is the transport and administrative headquarters of Rift Valley province (Foeken 2006; Owuor 2006). Just like Nairobi and Kisumu, Nakuru emerged from the 
construction of the Kenya-Uganda railway in 1904. It soon developed into an important regional trading and market center. It is located approximately $160 \mathrm{kms}$. Northwest of Nairobi, and is accessible from Mombasa and Nairobi by rail and road. According to Foeken (2006), the area where Nakuru town is situated used to be grazing land for pastoral communities such as the Maasai prior to the arrival of the Kenya-Uganda railway. He reports that the town was named Nakuru, a Maasai name that refers to "a place of winds," because it is located on the floor of the Great Rift Valley where it is usually engulfed by whirling winds of dust, especially during dry seasons.

Demographically, Nakuru draws from the majority of the 42 officially recognized ethnic communities in Kenya. The population size keeps on changing due to ruralurban migration (Mwangi 2002; Owuor 2006). The Kikuyu are the majority, followed by the Kalenjin. However, the population of other ethnic communities such as Luhya and Luo has grown significantly in the recent past (National Housing and Population Census Report 2009). Some researchers report that Nakuru's residential patterns are segregated along socio-economic lines. The high-income neighborhoods located to the north of the Kenya-Uganda Railway while the low-income neighborhoods are to the south (Foeken 2006). ${ }^{5}$ Both high and low-neighborhoods have people from diverse ethnolinguistic backgrounds but neither is occupied predominantly by particular ethnic communities (De Meulder 1998; Foeken 2006).

Several other researchers report that Nakuru has been experiencing periodic ethnic violence, especially during the period immediately before and after general elections (Kagwanja 2003; Klopp 2001; Waki 2008). This violence escalated after the 2007

\footnotetext{
${ }^{5}$ The Kenya-Uganda railway and Mombasa-Kisumu highway cut across Nakuru town.
} 
contested presidential election, which fueled preexisting interethnic tensions and led to displacement of people from targeted ethnic communities. These people either relocated to residential neighborhoods where they felt safe or to the rural areas, which are mainly ethnically homogenous. According to Waki (2008), the ethnic diversity in Nakuru area has been a source of tension and (ethnic) conflict, pitting the Kalenjin and Maasai communities who regard themselves as "insiders" against other communities such as Kikuyu and Kisii that they perceive as "outsiders." In this regard, Waki (2008: 82) reports that Kalenjin and Maasai usually refer to these “outsider" communities as madoadoa (Swahili for 'stains'), arguing that they can only be removed through violence (also see Daily Nation Newspaper, October $17^{\text {th }} 2013$ ).

Many Kenyans believe that the main driving forces behind this violence have usually been to "repossess" the land owned by the so-called "outsiders," and also to enhance ethnic homogeneity for the benefit of the political aspirants from the communities that perceive themselves as "insiders." According to Waki (2008), these "insiders" usually pursue the two motives alongside a desire for a federal system of government commonly referred to as Majimbo, 'federalism'. The proponents of Majimbo usually claim that their main aim is to devolve power to a level where there would be equitable distribution of resources for the benefit of the common citizen.

However, Waki claims that Majimbo in actual practice is a political tool for reducing political competition, especially in the ethnically heterogeneous areas in favor of the so-called "insiders." Consequently, violence has become a means of winning elections and securing political power in Kenya, a situation that perpetuates ethnic animosity between the many ethnic communities in the country. In 2007 , for example, Waki observes that the Majimbo debate in the period surrounding the 
general election was ethnically divisive. References to madoadoa were used frequently even in normal discourses within Nakuru and neighboring districts. Also, some of my informants told me that linguistic features such as accent and shibboleths were used to identify members of targeted ethnic communities during the 2007 postelection violence. In Nakuru, for example, Hora, a student in a focus group, reported how he and his friends witnessed members of a certain ethnic militia asking perceived outsiders to pronounce words with shibboleths in order to ascertain their ethnicity. According to Hora, members of this militia told their targets to pronounce the word

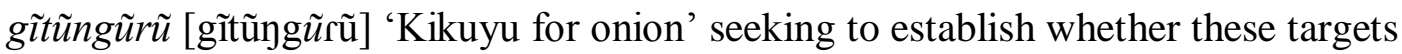
would pronounce the trilled /r/, failing which these individuals were lynched (Hora and Bakari, 10/2011, focus group-- Nakuru).

In addition, the Standard Newspaper (October $24^{\text {th }} 2013$ ) reports that one of the witnesses in the ongoing case about the Kenya's 2007 post-election violence at the International Criminal Court (ICC) told the judges that, "the attackers would ask their perceived victims questions using a code and if they could not answer, they would be hacked to death." Many of my informants in Nakuru reported that these ethnic conflicts affected their social relations with immediate neighbors in their residential neighborhoods and colleagues at workplaces. Mombasa, the second site for my study, had similar experiences in terms of ethnic animosity as the discussion below demonstrates.

\subsubsection{Mombasa}

Mombasa is an island along Kenya's "Swahili" coast, and is the administrative headquarters of Coast Province. It is one of the oldest cities in Kenya and is located approximately 485 kilometers south of Nairobi. It is easily accessible from other 
major towns such as Nairobi, Kisumu and Nakuru by road, railway and air transport. Mombasa is also an important port facilitating trade and transport networks between East African region and the rest of the world.

According to Abdul-Aziz (1979: 11), early historical accounts indicate that Mombasa was divided into two main parts, which are partially visible today: "the ancient city of Mji wa Kale, and the Portuguese-built quarter of Gavana, which constituted the administrative and commercial part of the town. A thick wall which has now disappeared separated the two quarters." Abdul-Aziz elaborates that Gavana was occupied by Arabs and was comprised of stone-walled houses with a huge street called Ndia Kии that cut halfway across. Gavana was significant from commercial, military and administrative points of view. Mji wa Kale was comprised of shanties and was occupied by the "Swahilis" (see discussion of this ethnonym in Chapter Four). Mji wa Kale was therefore significant because of the rich history and traditions of the "Swahilis." For example, Abdul-Aziz reports that the "Swahilis" claim that it was the home of their ancestors and regard it as a place of heroes where "real" and “authentic" Swahili called Kimvita is spoken. Consequently, speakers of Kimvita have often resented speakers of other Swahili varieties, both local and foreign. They have also consistently contested the choice of the Unguja dialect of Tanzania as the basis of the current standard as the national language of Kenya (Abdul-Aziz 1979; Mbaabu 1985; Nurse and Hinnebusch 1993; Wald 1973, 1985).

The above scholars report that Mombasa has also been in contact with the outside world and the upcountry communities such as Kikuyu and Kamba for many centuries. Due to its close proximity to the mainland, the $19^{\text {th }}$-century visitors such as the Arabs, Portuguese and British found it a strategic and important place for transport, 
commercial and administrative purposes. The Arab merchants, for example, traded with coastal communities and often intermarried and established new settlements alongside local communities including the Swahilis. Over time, new forms of Swahili language and culture emerged, especially among Swahili speakers of mixed racial and ethnic backgrounds, adding to the diversity of the Swahili-speaking community in Mombasa.

However, given that speakers of Kimvita claim to be the "real" Swahili, the question of who is an Mswahili has always been contested, creating fluid notions of insider-outsider categories (Eastman 1971; Wald 1979, 1985). On the one hand, the question manifests itself among speakers of various coastal varieties of Swahili. In this context, speakers of Kimvita claim to speak "insider Swahili" while referring to other varieties as "outsider Swahili" including the standard. On the other hand, there is also tension between speakers of coastal varieties of Swahili such as Kimvita and speakers of the upcountry varieties. ${ }^{6}$ In this coastal-upcountry opposition, speakers of the coastal varieties of Swahili claim to be the Waswahili and "legitimate" occupants of the coast, while they pejoratively refer to speakers of upcountry varieties as watokabara, 'people from upcountry'.

I found that similar tensions existed among young Kenyans at the coast; those from ethnic communities which regard themselves as coastal claimed to speak lugha ya mtaa, 'street language' while associating Sheng with speakers that they perceived as from upcountry, especially Nairobi. Even though many Kenyans, especially adults, use the label lugha ya mtaa to mean street language in general, young Kenyans who associated themselves with Mombasa employed the label specifically when referring

\footnotetext{
${ }^{6}$ These are Swahili varieties whose pronunciation, word choice and meanings are influenced by speakers' ethnic languages.
} 
to the nonstandard language variety that they spoke. They told me that it was different from Sheng and gave vocabulary words as examples. Thus, while there are contexts where some Kenyans would regard Sheng as lugha ya mtaa, the latter should not be misconstrued to be be another name for Sheng.

Just like Nakuru, Mombasa also experienced the 2007 post-election violence, which awakened underlying ethnic tensions and significantly affected peoples' relationships at home and in workplaces. These tensions were relevant to my research study since it coincided with a time when Kenya was in an electioneering mood. Many of the issues discussed in political rallies, and subsequent conversations among ordinary Kenyans were ethnically charged. My research sought to know how Sheng responds to such ethnically and regionally polarized environments.

In summary, Nakuru and Mombasa were appropriate sites for the present research because both were linguistically and ethnically diverse. They also provided social contexts with underlying tensions revolving around the questions of language, identity and nationalism, though in different ways. In addition, since many Kenyans associated Sheng with Nairobi, it was important to find out: 1) whether Sheng existed in other urban areas; and 2) whether speakers in the two research sites saw it as different or similar to that of Nairobi. In that regard, I sought to know whether they called it Sheng or if they employed different labels and why. I now focus on my actual fieldwork experience starting with Nakuru.

\subsubsection{Research in Nakuru (August to December 2011)}

I applied and received permission to conduct research in high schools within Nakuru from the local government authorities. I then rented a room in a hotel to allow myself time to shop for an affordable apartment in a safe and accessible residential 
neighborhood for my convenience during research. In choosing an appropriate school, I consulted with a personal contact that I will callSammy. He is a speaker of Sheng who attended high school in Nakuru and graduated in 2010. We started with a general tour of Nakuru town and various residential neighborhoods so that I could inform myself about local means of transport such as matatu, boda-boda and tuk-tuk. ${ }^{7}$

During this time, I engaged Sammy in Sheng conversations, seeking to update my own Sheng and learn about interests of young people in Nakuru. Given the poor state of the roads, riding in matatu, boda-boda and tuk-tuk was not comfortable. However, these rides were an important research experience since these forms of transporatation are some of the social spaces associated with the use, production and reproduction of Sheng and other nonstandard language varieties such as lugha ya mtaa in Mombasa. Also, the tours helped me learn about local schools and marketplaces frequented by young Kenyans, especially high school students. These places included cybercafés, shopping malls, sports fields, music stores and public parks. By the beginning of the first week of September, I had identified three schools whose general description and location seemed to fit my research project. Top on the list was Someni High School which later became my actual research site for various reasons outlined below.

\footnotetext{
${ }^{7}$ Samper (2002) defines matatu as privately owned vans that provide public transport in Kenya. They are usually known for the flashy decorations and graffiti on their body in addition to playing loud music that appeals to the youth. The operators of these matatus (touts) are called manamba (Swahili word for number but with $m a$ - prefix to mark plural). Manamba call out the route numbers, inviting passengers to board based on the routes and destinations where a given matatu is plying its trade. Many manamba are school dropouts and they are reported to be the main innovators and cultural brokers of Sheng together with hip-hop artists (Githinji 2006; Samper 2002). Boda-boda is a Swahilized term derived from English "border-border" and is used in East Africa to refer to a bicycle or motorcycle taxi (see Kisaalita and Sentongo-Kibalama 2007). Tuk-tuk refers to a mechanized three-wheeled taxi mainly found in major cities in East Africa and India.
} 


\subsubsection{Location of Someni School}

The school is located within Nakuru town on the eastern end along the busy Nakuru-Kisumu road. It has a perimeter wall separating it from the neighboring marketplaces. Its main gate is manned by a watchman who controls and documents the flow of students and visitors in and out of school. The school borders the main farmers' open air market on the northern side while on the western side there is a mitumba, 'secondhand clothes' market. There are also music stalls within the mitumba market, which sell and play different sorts of music. The mitumba market separates the school from some busy malls and one of the main matatu terminuses in Nakuru. On the southern side is a tarmac road along which the main school gate is situated. The tarmac road separates Someni School from privately-owned residential quarters. Along this road are small-scale businesses such as food kiosks, boda-boda operators, newspaper vendors and shoe-shiners. The road is also served by several matatu, ferrying passengers to and from different residential neighborhoods within Nakuru town. On the eastern side is an open grass field. This field separates the school from a small shopping center that serves the residential neighborhoods to the east and south sides of the school. It is common to find street boys in this field seated in small groups chatting, playing cards, sniffing glue or just lying on the grass.

Despite having a perimeter wall and a main gate demarcating the school from the neighboring marketplaces, students, teachers and other members of staff are in constant contact with the highly multilingual environment of Nakuru town. They interact with speakers of Sheng and other nonstandard language varieties not authorized for use within school settings. For example, I observed that teachers and students often use matatu, tuk-tuk and boda-boda to and from school. They also visit 
nearby markets to buy various needs such as food. Some of these traders are friends and relatives of students and teachers of Someni, indicating that students and teachers belong to other social networks outside the school. Next, I focus on the population and diversity of the school.

\subsubsection{Population and diversity of Someni}

The school is ethno-linguistically diverse and co-ed. It has more than 900 students, slightly more boys than girls. The students are drawn from different socioeconomic backgrounds. However, the majority is from the low-income residential neighborhoods that some of my informants described as ghetto or slums. There is also a small population that comes from the neighboring rural areas, approximately ten kilometers from Nakuru town. From an academic point of view, the government categorizes Someni as a provincial school, meaning that it admits students who excelled in Standard Eight (grade eight) national exams, but did not qualify to be admitted to a national school. The term "National School" in Kenya refers to schools which admit students with the highest grades in Standard Eight exams nationwide. However, some teachers told me that there were students who had qualified for national schools but their parents brought them to Someni for financial reasons.

The school staff and student population is also diverse from a religious point of view: it has Muslims, Hindus, and a Christian majority. Just like the student population, Someni has a big staff of approximately 70 teachers and 40 nonteaching and subordinate staff who are ethno-linguistically diverse. However, Kikuyu are the majority. Some informants told me that a big fraction of the Kikuyu relocated from other parts of the Rift-Valley Province following the 2007 post-election violence. 
Apart from two or three teachers who live in the school compound, others live in various residential neighborhoods in the outskirts of Nakuru town. There is also a small fraction of teachers who commute from the rural areas. These aspects of diversity and mobility were relevant to my research since the study sought to compare language use patterns between school and out-of-school settings. This comparison aimed at establishing whether respondents associate certain languages with schools and others with out-of-school settings, and illuminating the root cause of the disconnect between the teaching of the officially sanctioned Standard Swahili and English in schools and their use in actual practice. I now discuss how I undertook the actual research at Someni High School.

\subsubsection{Commencement of my research at Someni}

I visited Someni School on a Friday morning around mid-September. I went to the Principal's office and introduced myself, described my research and sought his permission to conduct research there. I also presented the authorization documents from the various government agencies and a letter of introduction from my dissertation advisor. The Principal engaged me in a general conversation seeking to know more about me and why I chose his school. Considering that use of Sheng is discouraged in schools, I used the information I had gathered from my contact person and some of my friends who were former students there to explain why I chose Someni. I kept the focus of my research in this conversation on language and student life in general. At the end of our conversation, the Principal granted me permission, then asked me to come back the following Monday to be introduced officially to the whole school during morning assembly. 
I returned on Monday morning as we had agreed and found the Principal in his office. When the bell for morning assembly rang, we both proceeded to the assembly ground where we met students and other teachers. The Principal asked me to stand next to other teachers as he proceeded to the podium. He started his speech addressing various issues affecting the school such as the national teachers' strike that was occurring at that time. The teachers' strike became relevant to my research because though teachers reported to the school, there was no teaching going on. Regardless, students were still expected to report to school and remained in classrooms. Teachers showed solidarity with strikers by not teaching. However, since Someni performs well in national exams teachers felt a responsibility to their students, so they showed up and stayed in their offices. It is in this context that the Principal saw the need to provide guidance to the students on how to behave and carry on with their studies since the national exams were a month away.

When my moment to be introduced came, the Principal did a magnificent introduction, emphasizing every aspect of my identity in a way that left me wondering whether it would negatively affect my research. Part of the introduction was that I was young Ph.D. student of anthropology in the U.S. who had come to Someni to do his doctoral research on language. He also told them that I used to teach Swahili in high school before I left for the U.S. He concluded by asking teachers and students to embrace me and accord the necessary support during my research. While the Principal aimed at inspiring his students, I worried that his introduction projected an image of a person of high status that students would not feel comfortable interacting with closely. Interestingly, I observed that students expressed different reactions to the various aspects of my identity. Specifically, they seemed amazed when the Principal 
mentioned that I was a young Ph.D. student in the U.S. However, they chuckled when they heard that I used to be a teacher of Swahili in high school. As later discussion of my findings will show, the contrast in their reactions alluded to the global-local tension in their negotiation of identity. It also alluded to their differential evaluation of English and Standard Swahili, despite the fact that both are official languages in Kenya.

After the assembly, the Principal introduced me to one middle-aged teacher, Mr. Gutz, and asked him to be my cooperating teacher. ${ }^{8}$ Mr. Gutz taught Swahili and English and was also the patron of the school Drama Club. This marked the beginning of my preliminary observations. In the following section, I outline the main initial activities of my research at Someni School.

\subsubsection{Familiarization tour of the school}

Mr. Gutz took up his role as my cooperating teacher immediately after the assembly. He took me to different social spaces such as the teachers' lounge, dining hall, playing ground, library, laboratories and departmental offices. He also reintroduced me to various subject teachers that we met in these spaces. In addition, he took me to various classes where he taught Swahili and introduced me to his students. Recall from my discussion above that no teaching was taking place at this time because of the ongoing national teachers' strike. Instead, teachers stayed in their offices while students studied on their own. Interestingly, Mr. Gutz told these teachers and students that I was a Ph.D. student studying and teaching Swahili at a university in the U.S. Sometimes he would get flamboyant, emphasizing that I taught Swahili to

\footnotetext{
${ }^{8}$ In Kenya, the term "cooperating teacher" is used to refer to a member of the teaching staff who voluntarily or is officially asked by the school administration to help newly-posted teachers or those on teaching practice settle and learn the school environment.
} 
Americans and other times saying wazungu, 'whites'. ${ }^{9}$ He also mentioned that there were Kenyans in the U.S. pursuing Swahili. He would conclude the introduction by asking the students whether they would like me to visit them again and to tell them the benefits of studying Swahili. Students often applauded the idea and sometimes encouraged Mr. Gutz to leave so that they could have time with me. This became the routine for the first few days.

A critical look at Mr. Gutz's introduction indicates that he seized the moment to popularize and enhance the status of Swahili, an effort which could have affected students' and teachers' perceptions about me as a researcher. From the negative end, mentioning that I was a teacher of Swahili could have created unnecessary social distance with my potential informants because it automatically invoked the unequal teacher-student power relations. Also, emphasizing that I taught Americans or wazungu could have created a very superficial sense of who I was, making my potential informants uncomfortable when interacting with me. These worries became evident later in the research when some students confessed openly that they feared talking to me in the early days of my research because of how the Principal introduced me on the first day. Others reported that they feared talking to me during the days that I put on a suit because they associated such clothing with teachers or people in authority.

Despite these potential drawbacks, Mr. Gutz's introduction still yielded some benefits for my research. Some students who believed that I was an expert of Swahili asked me some questions which turned out to be nice "hooks" for initiating

\footnotetext{
${ }^{9}$ Teaching Swahili in the U.S and to wazungu should be understood in the context of being an expert in the language, because many Kenyans regard Swahili as a language that does not promise career prospects beyond East Africa.
} 
discussions in the focus groups. For example, one student, Kadonye, in a Form Two class, asked me: “Mbona Kiswahili ni kitamu lakini kigumu?”, 'Why is Swahili sweet but difficult?'

Given that students in Kenya are socialized to believe that a teacher knows a lot and is always ready to teach, I must confess that there were awkward moments for me at the initial stages of my research in Nakuru. However, since Mr. Gutz would leave me with the students immediately after the introduction, I came up with a strategy to save face and that would not jeopardize my research. Instead, I would steer their focus away from my identity as a Swahili teacher by asking them general and open-ended questions such as the languages they studied in school, their knowledge about the U.S. and what they did outside class. The discussions that followed helped me socialize and build rapport with these students.

In summary, Mr. Gutz's tours and class visits helped me familiarize myself with various social spaces within the school compound and also gain legitimacy as a researcher among teachers and students. In the long run, I was able to interact with my informants in the absence of Mr. Gutz and to easily get their consent during various research activities such as focus groups, interviews and classroom observations. Since I employed the same research procedures and data collection methods in both Nakuru and Mombasa but had different research experiences, I will switch to a discussion of my research experience in Mombasa, and then return to the research procedures thereafter.

\subsubsection{Research in Mombasa (January to April 2012)}

I left Nakuru for Mombasa in January, 2012. I applied and got permission from the local government authorities to conduct research in local schools. I also linked up 
with one of my contact persons, Gathiari, whose family is from the upcountry but who had lived in Mombasa for many years. He helped me learn how to navigate the city using local means of transport, tuk-tuk and matatu. He also gave me a tour of Mombasa, especially marketplaces usually frequented by young people. In addition, he introduced me to some of his male friends who lived and operated taxi businesses in Mombasa. As I socialized with these men, I asked them about the languages spoken in Mombasa and whether there was a language associated with young people. Interestingly, many of them told me that people in Mombasa, including young Kenyans, spoke Swahili. At some point, I specifically asked them whether there were people who spoke Sheng in Mombasa. They responded that there was no Sheng there and if I was really interested in it, I should have gone to Nairobi where I would find plenty of it. These responses were at first discouraging since I had come from Nakuru where I had identified Sheng easily. Nevertheless, I kept reminding myself that the absence of Sheng would still be interesting for my research.

By the end of the first week, I had rented an apartment and informed myself about the local schools. There was only one school on Mombasa Island, Rohoni, which matched my previous research site in Nakuru. All the others were public single-gender or private co-ed, day and boarding high schools. Some of my Muslim informants told me that the small number of co-ed public schools in Mombasa was due to Islam, which discourages young boys and girls from interacting freely (Mr. Shehe 01/2012, interview). To get a good match with Someni School in Nakuru and one that had a student population representative of Mombasa's population, I decided to start with two co-ed schools-- one private and one public ${ }^{-}$- which were ethnolinguistically and religiously diverse. I will call the private one Vikapuni while the 
public one is the Rohoni mentioned above. The choice of the two schools was also motivated by the desire to find out whether there were significant differences between private and public schools in Kenya, which would affect how speakers perceive and employ language in their day-to-day interactions. Next, I focus on the location of the two schools.

\subsubsection{Location and diversity of Rohoni and Vikapuni High Schools}

Rohoni is located on the western side of Mombasa while Vikapuni is on the eastern side. Both are on Mombasa Island and are served by matatu and tuk-tuk plying their trade across the town and along the main Tudor-Docks road. Just like the Someni School in Nakuru, despite having a perimeter wall and a manned gate, both schools are in close contact with the outside communities. I was therefore able to investigate how language use in school settings articulates with speakers' language use patterns outside the school. Both schools have an ethno-linguistically and religiously diverse population of more than 600 students and more than 25 teachers. These students are drawn from communities which are perceived as coastal and from those perceived as from upcountry. As mentioned earlier, this distinction between coastal and upcountry communities is relevant to my research because it plays a significant role in peoples' definition of who is an Mswahili, which also comes up among young Kenyans in Mombasa. From an academic point of view, while Rohoni is a District Day High School that performs poorly in national exams, Vikapuni is a private Day and Boarding High School whose students excel in national exams. It would fit well in the provincial category if it were public. I now turn to the actual research experience in both schools. 


\subsubsection{Commencement of my research at Rohoni and Vikapuni High Schools}

I started my research in the second week of January, immediately after school resumed from the December holidays. I visited the Principal of Rohoni and followed the same introductory procedures as I did in Nakuru. However, she was hesitant to grant me permission to conduct my research there. Instead, suggested that I go to a different school, with the excuse that many of her students would not be available since she was going to send them home for school fees the following day. Even after I explained to her that Rohoni was the only school in Mombasa that matched my previous research site in Nakuru, she was still not willing to allow me. Instead, she asked me to come back after a week to see whether her students would have settled down for the term/semester. I complied and promised to come back. I then left to see the Principal of Vikapuni High School, which was my alternative. I met the Principal of Vikapuni in her office; she was warm and friendly but said that she needed to consult with the school Board of Governors since Vikapuni was a private school. She advised me to leave my contact information with her secretary and promised to contact me as soon as she talked to the Chairman of the Board. I spent this waiting period visiting different marketplaces and collecting materials such as magazines, street graffiti, advertisements, local newspapers and comics that I would use to initiate discussions in my interviews and focus groups once I started my actual research.

I went to check with the Principals of both schools after a week and they granted me permission to conduct research. In each of the two schools, I was given a "cooperating teacher," who then introduced me to other members of the staff and gave me a tour of the school. As mentioned above, the reason for selecting Rohoni was to get a good match with Someni School in Nakuru. I also wanted to find out whether 
the private-public distinction had any significant influence on how people employ language. Therefore, I decided to visit both schools daily, one in the morning and the other in the afternoon, for preliminary observations. I planned to later settle on the more appropriate one. I conducted preliminary observations in both schools for the whole of January but focusing more on Rohoni because it was a perfect match with Someni School. I started interviewing teachers, sat in select classes and began forming focus groups with students. To gain access to students, I assisted the volleyball coach in training the team. Volleyball became very popular, and many students started to show up at the training.

On the first Monday of February, I arrived at Rohoni only to find the situation had changed drastically. As soon as I greeted staff members and sat down, the secretary came and informed me that the Principal wanted to see me. I was not aware of any mishap from the previous week but I could tell from the faces of the other members of staff that all was not well. So, I walked to the Principal's office and found her with her deputy. Immediately after I greeted them and was offered a seat by the Principal, she informed me that she hadn't known that my research would involve coming to school daily and interacting with teachers and students that much. The Principal also denied having granted me permission to do research at her school. In addition, she claimed that the kind of students she had seen me interacting with were those with discipline problems, especially volleyballers. ${ }^{10}$ She then asked me to reapply for her permission to do research in her school in writing and she set very

\footnotetext{
${ }^{10}$ Many school heads and teachers in Kenya associate extracurricular activities with students who do not perform well academically and sometimes those with discipline problems. This may be partly why the Principal of Rohoni High School could not approve of my presence there. In fact, my dismissal may also have been fueled by the fact that she had asked me to teach Swahili but declined since the identity of a teacher could have affected students' perceptions toward me as a researcher.
} 
stringent conditions for me. These included: I should only be coming to school on specific days and during break times, that is class recess; I should avoid interacting "too much" with her teachers and instead I needed to identify a place outside the teachers' lounge from where I should be doing my observations; if possible I should administer questionnaires to minimize interaction with the teachers; finally, she also told me not to be eating the food and tea provided to the teachers since I was not teaching any lessons for them.

It took four days for her to grant permission, even with these conditions. Once I resumed, I was told by one of the teachers that the school was going through serious administrative challenges, hence the Principal was not comfortable with outsiders seeking to interact closely with teachers and students for fear of leaking information to the government. Whatever the reasons, I had at this point established that the private-public distinction did not have any observable influence on language-use patterns. I had also foundthat students' friendship networks were based on grade level and were organized in social groups just like in Nakuru. So, after two weeks I dropped Rohoni and concentrated on Vikapuni High School for the rest of my research since it provided a more conducive environment and the same quality of data as I stood to glean from Rohoni.

As mentioned earlier, the administration of Vikapuni was welcoming. As such, I embarked on building more rapport with teachers and students. This became even easier after I started going to the school regularly and participating in extra-curricular activities such as volleyball. In addition, I started attending Wednesday church services where I was able to interact more with teachers and students while doing my observations. Some teachers even invited me to their homes for lunch and Sunday 
services in churches where they served as pastors. Within a very short time, I was able to gain required acceptance among teachers and students which enabled me to start forming focus groups, observing selected classes as well as conducting individual interviews. As mentioned earlier, I employed the same research procedures and data collection methods in both Nakuru and Mombasa. I discuss each of them below in detail.

\subsubsection{Data collection}

I originally planned to give recorders to students to audi-record one another without my presence in order to collect near-natural speech. However, my preliminary observations and interactions with students indicated a possibility of either damage, loss of equipment or unethical or surreptitious recording of peers. As such, the ethnographic examples in this dissertation are mostly conversations where I was the interlocutor, as opposed to conversations that I observed without being a participant myself, or observations of what were happening in classrooms.

\subsubsection{Identifying/recruiting informants}

For the teachers, I applied a combination of strategies in the identification and recruitment of participants. I started by spending time in their lounge observing and noting down the languages they spoke in their day-to-day interactions. I also engaged them in casual conversations about general issues in an attempt to build rapport with them (see below). I would then seek to have a follow-up interview with the individual teachers who made statements or comments relevant to my research. For example, I used to position myself at a strategic place in the teachers' lounge reading one of the daily newspapers to draw the attention of those entering the lounge. It was almost impossible for such teachers to pass me without asking what news was featured that 
day. Over time, I discovered that the Swahili language newspaper called Taifa Leo (as opposed to the English language ones called the Standard and Daily Nation) used to draw attention from many teachers who would make interesting comments relevant to my research. I would note down such comments and later seek to interview these teachers.

Another strategy was to directly approach teachers of specific subjects such as Swahili or English and request to sit in their classes to observe how they and their students employed language in their interactions. I would later seek a follow-up interview with them for more information about the observations I noted. Also, some teachers would refer me to their colleagues whenever I raised a question that they thought would be better answered by a particular teacher.

In summary, I was always on the lookout for potential teacher informants, hence I applied multiple strategies to identify and recruit them. However, there were some teachers in Nakuru who were suspicious of my presence in the school, especially because my arrival coincided with the national teachers' strike. Therefore, some teachers thought that I was a government spy, investigating what they were doing or saying about the government and therefore interacted with me cautiously. Our interactions improved, however, after the strike was called off and they started honoring my requests to observe their classes and for follow-up interviews.

In the case of students, I started by inviting individuals who made interesting comments in the classes that I observed, for casual conversations about what they said in class or general issues about the school. I would also ask them about their friends, aiming at establishing how students formed friendship networks. Where an individual 
student mentioned that s/he belonged to a particular social group, I employed snowball sampling. This is a research method where identified respondents help in recruiting more subjects from among their acquaintances (Biernacki and Waldorf 1981). I would ask the identified student to describe his or her social group, seeking to know what their common interests were and how they defined themselves. I would then request him/her to invite other group members for a casual conversation at a later date. At first, it was hard to get access to these students because they feared that I might betray them to the school administration. However, the more I interacted with different categories of students, the more students in general became comfortable with me.

There were also times that I would find a group of students in the playing field during tea or lunch break and introduce myself. I would greet them and initiate a casual conversation seeking to build rapport. Whenever I succeeded in doing so, I would invite them for a later conversation about some observations related to my research. Given that many students were interested in knowing about the U.S., I succeeded in arranging many such meetings. In some other cases, I would ask a teacher whom I established was popular among students to take me to the school canteen with the pretext of buying him/her a soda, while actually I wanted to gain access to a group of students I had seen at the canteen. Once we got to the canteen, I would greet these students, especially if they were in a small group, and buy them sodas together with the teacher. In such cases, it was almost automatic that these students would want to talk to me, seeking to appreciate my kind gesture. I would then seize the moment to engage them in a casual conversation about general issues, aiming to find out whether this was a social group of friends that I would invite for a 
conversation about my research. Further, there were situations where some teachers referred me to particular students, especially classroom monitors and leaders of particular student clubs and associations such as the Praise and Worship. (I found ways of locating other students besides the ones approved of by the teachers.)

Just as in the case of teachers, not all students agreed to participate in my research. In such situations, I would steer away from them and focus on others. So, the aim was to talk to as many students and teachers as possible in the initial period of my research, to create a large and diverse pool of informants. This helped in enhancing acceptance among teachers and students, which further made my presence inconspicuous especially during class observations and in the teachers' lounge. In addition, the strategy ensured that I got informants to participate in the interviews and focus groups even when the school learning-and-teaching schedule got busier. I now turn to the data collection methods.

\subsubsection{Casual conversations}

This strategy helped to simultaneously identify informants and to gather "hooks" for use in the interviews and focus groups. Besides that, I used casual conversations mainly as ice-breakers and building rapport with informants. As such, many of these conversations focused on general issues such as those captured in the daily newspapers, teaching subjects and how each school performed in national exams. My informants, both teachers and students, would in turn ask me about various issues such as life in the U.S. how to get educational scholarships or about individuals such as president Obama. Some teachers would ask me about my research 
and degree of study, especially because many of them did not know much about Anthropology.

Since casual conversations occurred in unstructured settings, they produced the most natural speech and relevant rich points. For example, it was common for teachers to praise their school's general academic performance, but immediately point out that their students' performance in English and Standard Swahili has been affected by Sheng. Others would employ statements in their conversations that would qualify as Sheng. Depending on the prevailing conditions, I would employ different strategies to note down such spectacular moments. There are times I openly told my interactants that I would like to write down what they said. However, if I realized that they were uncomfortable, I would excuse myself after a short while to go and make notes in private. After sometime, my informants got used to my note-taking and were no longer bothered when they saw me writing something. I would later seek to interview the teachers I talked to and where possible, audio-record these interviews for later analysis. Another data collection method I employed was direct observations, which I discuss below.

\subsubsection{Direct observations}

I conducted direct observations in various spaces within and outside the school, from the beginning to the end of my research. Within the school, I visited the teachers' lounge, sat in selected classes such as Swahili and English, attended club meetings, and participated in student training sessions in games and sports. I also toured various blocks and classrooms where there were notice boards, and took note of the languages used in the posted material. Sometimes, I would request one or two 
students to accompany me on such tours and ask them to comment on the languages used. Whenever I found notices, memos or posters with notable linguistic forms, I would request the concerned teachers to give me copies of the same, assuring them that I would make good use of them.

Outside the school, I could not interact with students since the school authorities prohibited me from doing so. Nevertheless, I still made observations that were relevant to my research. I used to spend time in the streets, and in marketplaces such as malls and music stores, observing the languages that people employed in their conversations, noting them in my note book. I would also note down the words or expressions used in advertisements and commercial posters displayed on the walls of buildings. I would then use these observations to elicit comments from my informants once I went to school.

In sum, direct observations provided "hooks" for initiating discussions during casual conversations, interviews and focus groups. These discussions would then reveal speakers' attitudes toward various languages, and whether they associated particular languages with certain spaces. For example, it emerged from the research in both Nakuru and Mombasa that students associated Standard Swahili and English with school and exams, rather than media for day-to-day communication and/or socialization. Even within the school, students associated various language varieties with certain people and spaces but not others. For instance, they told me that they did not expect the Principal to address the school assembly in Swahili or to speak Sheng. However, I later found out that there was variation in peoples' language use in these contexts associated with exclusive use of particular languges. This variation suggests that there was a disconnect between what informants reported or believed to be doing 
and their language use patterns in actuality. I attribute this disconnect to the fact that speakers' language choices were ideologically-mediated.

\subsubsection{Audio-recorded individual interviews}

I conducted individual interviews with both teachers and students. However, this method worked better for teachers because of their busy teaching schedules, which made it difficult to interview more than one teacher at a time. Also, some teachers were not comfortable discussing some issues about the school in the presence of their colleagues, for fear of being implicated or perceived as saboteurs by the school administration. Others were so opinionated that I thought it would be hard for them to accommodate other peoples' opinions. In addition, individual interviews provided more time and space to make in-depth follow-ups with particular teachers about some interesting observations or claims they had made in group settings such as in the teachers' lounge.

However, not all teachers agreed to be interviewed. Some either referred me to their colleagues while others agreed but never committed themselves to an appointment. The issue of honoring appointments is relevant to my research. I came to the realization that as a cultural trait, many Kenyans are generally uncomfortable with formal arrangements. This potentially affected my research since it was hard to follow a systematic, predictable research plan. There were teachers who agreed to be interviewed but got uncomfortable and even rude along the way, prompting me to terminate the interview. Even though such reactions were discouraging, I later learned that such teachers had had nasty experiences with previous researchers, and were therefore not willing to be the subjects of research again. The reactions also revealed that such teachers had a limited understanding of ethnographic research. Some told 
me that they were used to researchers who would come to school and distribute written questionnaires to be collected at a later date. Recall the Principal from Rohoni, who expected me to distribute questionnaires rather than interacting with teachers and students. In fact, some teachers in Nakuru seemed to expect my research to be concluded in a very short time, and on seeing me would ask "Hujamaliza bado?", "Haven't you finished yet?” Further, some teachers were generally nervous and could not sustain a conversation, possibly because they felt inadequate or insecure addressing some of the issues I raised. In such situations, I had to terminate the interview tactfully to save them more embarrassment. Given these conditions, I managed to interview and audio-record 11 teachers in Nakuru and ten in Mombasa for approximately 23 hours total.

In the case of students, individual interviews did not work well because many felt like they were being interrogated. As a result, they often became self-conscious about why I singled them out. Some were also concerned about how other students would think of them, fearing being misconstrued as traitors. As was with some teachers, some students felt inadequate or insecure commenting on some issues. Thus, they preferred speaking in a group setting where they could seek approval from peers, or have other people to share the burden of providing the information sought.

However, I still managed to conduct a few individual interviews, some of which were very productive. These were mainly follow-up interviews with particular students of interest that I had identified either in casual conversations or in focus groups. In such a case, the student felt comfortable because s/he was already familiar with the topic and because we had established some rapport prior to the interview. I conducted two audio-recorded individual interviews with students in Nakuru and four in Mombasa 
for an approximate 5.25 hours in total. The difference in the number of interviews was due to availability of time and informants.

\subsubsection{Audio-recorded focus groups}

I employed ethno-linguistically diverse focus groups in an attempt to make my Kikuyu ethnicity less conspicuous. Also, it was my considered view that the presence of peers, especially among students, could create a more relaxed atmosphere than oneon-one interviews with an outsider, such as a stranger or an older researcher as Labov (1972) found. Like Labov, I would provide snacks and drinks to make the setting less formal, and we would often sit or even lie in the grass while talking. However, this method did not work well for the teachers because of the reasons outlined in the subsection above. Therefore, I employed focus groups among students only.

At the beginning of my research in Nakuru, I sought to find out the factors around which students formed their friendship networks. I also sought to know these factors influenced the way informants used and perceived the various language varieties they spoke. I established that grade level and gender were significant factors while ethnicity was not. With regard to grade level, there were tensions between students from different grades since the student organizational structure ranked Grade 9 [Form One] lowest and Grade 12 [Form Four] at the top in terms of seniority. It was therefore rare to find students from different grades spending time together. So, gender became my center of focus in each of my focus groups, drawing members from a given grade level. I formed two focus groups that I convened numerous times: one was boys-only and drew members from Form Three, while the other was mixedgender and drew students from Form Two. I also had several focus groups drawing participants from Form Four. Some of these foucs groupswere boys-only, while others 
were mixed-gender. However, these Form Four focus groups met only once because participants were busy doing national exams.

Even though different informants, especially in casual conversations, had claimed that boys and girls spoke different varieties of Sheng and that boys spoke Sheng more than girls, data from these focus groups did not reveal such differences. Instead, the data indicated that both male and female speakers' stances (Jaffe 2009) toward Sheng were contextual. ${ }^{11}$ These stances were influenced by the individual speaker's membership in multiple social groups with competing values and interests.

In Mombasa, it was not possible to convene the same focus groups numerous times because the school had a very busy schedule. However, I recruited several boysonly and mixed-gender focus groups. I drew participants from the same grade since I had already established that grade level was one of the main factors around which students formed friendship networks. Therefore, I aimed at speaking to as many informants as possible, seeking to know their social groups.

Overall, I conducted ten focus groups in Nakuru and 11 in Mombasa, approximately 21.5 hours total. For reference purposes, I have named these focus groups in two main ways. For those with two participants only, I have put the following details in parentheses: names, date and research site. For example, a focus group with students called Hora and Bakari in Nakuru would be referenced as (Hora and Bakari 10/2011, focus group--Nakuru). For the focus groups with more than two students, I have indicated in parentheses the grade, date and research site. For example, a focus group drawing students from Form Four would be referenced as (Form Four focus group, 11/2011--Nakuru). In situations where I had more than one

\footnotetext{
${ }^{11}$ Jaffe (2009: 1) defines stance as the orientation achieved by "taking a position in relation to the form or the content of one's utterance."
} 
focus group from the same grade, I have indicated the number of the focus group with a numeral, e.g., (Form Four focus group 2, 11/2011-Mombasa). The only focus group that has been named differently is the Praise and Worship in Nakuru. It comprises students from Form Four who were also members of the students' Christian Union.

I initiated group discussions by providing newspaper cuttings or posters from notice boards and asked participants to comment on the language(s) used. I would also ask them general questions such as the languages spoken in their school. I would then narrow down to specific questions, such as whether there was a particular language variety associated with young people in that area. In addition, I would seek comments about observations that I had made from other social activities within and outside the school such as sports, church services and dormitory weekly meetings.

I found focus groups more productive than casual conversations and individual interviews among students, because the attention and burden of providing information was shared among peers. The focus group setting also provided individual students with time to think about the questions I posed while others contributed. So, coparticipation and co-construction of meaning were central to students' participation in my research. The focus group setting also helped in recollecting memories about past events because students would piece together parts of what each one of them remembered about a particular event, and make it a meaningful whole. For example, whenever I asked about well-known social groups in the school or a past event, participants of the focus group would throw in single sentence descriptions which would then add up to a full description of the group or event in focus. Participants also kept seeking each other's approval. This was unlike in individual interviews where the interviewee would omit bits of relevant information either because $\mathrm{s} / \mathrm{he}$ was 
nervous, forgot some information or did not witness the incident in its totality. Focus groups also encouraged students to challenge each other's opinion, thus revealing how different categories of speakers defined, perceived and employed the various language varieties spoken within and outside school. This shared environment also helped in drawing participants' attention away from the audio-recorder.

\subsubsection{Students' written exercises}

I requested several science and language teachers to provide a few exam papers and class assignments that they had graded in order to see what "errors" they routinely corrected. For the sciences, I observed that teachers focused more on the appropriate use of technical terms than grammaticality of the language used. In the case of Swahili and English, I observed that many of the corrections were grammatical such as inappropriate use of verb tenses. There were also "errors" resulting from mother-tongue influence influence such as Kikuyu in Nakuru and Kimvita in Mombasa. Another correction that was common was the use of the nonstandard habitual suffix - anga to Swahili verb stems. Interestingly, very few teachers attributed any of these "errors" such as the suffix - anga to Sheng but the majority of them often blamed Sheng for their students' poor performance in Swahili and English exams. This suggests that Sheng is a social construct rather than a clearly defined linguistic form (see Chapter Two).

\subsubsection{Electronic and print media}

I collected comics, school magazines, newspaper cuttings, business posters, memos, printed school language policies, copies of school rules and regulations and and morphed photographs with Sheng captions. I also took photos of some research 
informants with notable features associated with Sheng speakers such as a modified uniform. I used some of these materials to initiate discussions or seek comments from informants during interviews and focus groups and to develop my arguments for this dissertation (see Appendix A and B for samples).

\subsubsection{Data management and analysis}

To identify follow-up questions, I went through my hand-written scratch notes daily or in a few cases after a few days. I then generated descriptive notes and save them in my external storage drive for later analysis. There were also moments when I wrote notes from memory, especially if something that I had observed only made sense after a few days. For audio-recorded interviews and focus groups, I would download and save them in my external hard drive at the end of each day. This was very important to avoid deleting files from the recorder before I had made copies. I would then play and listen to them and note down the emerging issues in my notebook for follow-up. This mode of operation helped me seek different peoples' points of view about the same issues. Sometimes, these opinions would form a pattern of themes in my descriptive notes from which I later developed my dissertation chapters.

I did not employ a systematic data coding system. Instead, I sorted my data manually where I would listen to several recordings per day and identify commonly occurring themes. I would then narrow down and transcribe these audio files, then write analytical notes. It is from these analytical notes that I figured out the main arguments to make and develop them into a chapter. Once I got a roadmap for a chapter, I would listen to more audios for additional material to reinforce my arguments. Even though it was a tedious process, I did not have a better option given 
the time pressure and financial and time constraints under which I conducted the research. However, I acknowledge that a more systematic data coding system could have been better in terms of data management and final analysis.

\subsubsection{Communication with my dissertation committee}

I periodically emailed detailed updates to my committee members about my experience and progress while in the field. This communication helped me remain focused. This orientation was especially helpful given the alienating nature of fieldwork experience, more so when working in an unfriendly community like the one I found in Mombasa. Some committee members would comment on my findings and encourage me to pursue certain observations by seeking more comments from different informants. This communication with the committee motivated me to keep asking questions and pay more attention to some observations that might otherwise have gone unnoticed.

\subsubsection{My biases as an ethnographer}

Despite my desire to be objective duringfiedwork, documenting, analyzing and representing the views of my informants, I struggled with my own biases. I echo Singh (2011: 19; also Haraway 1995) who argues that,

\footnotetext{
When working with other human beings, every researcher has a particular position in relation to the human beings from whom he or she learns whether it be with or against them (or both) — and whether he or she calls them informants, participants, interlocutors, friends, enemies, or something else.
}

I take this subjectivity and the ability to acknowledge it as strength; any researcher's work is not value-neutral, primarily because every method in which knowledge is produced and situated has its own biases (e.g., Singh 2011, Haraway 1995). In this 
regard, despite the rigorous training on how to conduct ethnographic research that I received at the University of Virginia, my experience during fieldwork brought out some of the identities that I have acquired in my life with their competing values and interests. These identities affected the way I interacted with my informants and perceived their responses to my questions. I analyze some of these identities and associated biases below.

Since I am a former high school teacher, I sometimes became conscious of the teacher-student relationship when interacting with student informants. This consciousness affected my choice of language and the way I employed that language in our conversations with regard to word choice, tone, and "correctness." It also affected how I judged students' feedback to my research questions. In addition, I became conscious of how I carried my body in our interactions, such as personal distance, sitting position, dressing, and emotions. It was therefore impossible to remain objective throughout my research. More specifically, given that I have been teaching Standard Swahili for the last ten years, it was sometimes hard to speak Sheng to my informants. Similarly, it was hard to accept Sheng speakers' arguments that Sheng is communicatively better than Standard Swahili. This was perhaps because I had an economic stake in protecting Standard Swahili by speaking it "correctly." That is, despite the fact that I study Sheng, I have earned my living through speaking Standard Swahili and English.

My regional and ethnic identity also affected the way I interacted and perceived my informants. Some of their narratives about the 2007 post-election violence projected Kikuyu as a threat to other ethnic communities in Kenya, hence potential targets during ethnic clashes. This identity threat was true in Nakuru as was 
in Mombasa, where people from upcountry, especially Kikuyu, were targeted for elimination by the communities that perceive themselves as coastal. Some of these narratives were very frightening because they were about how informants experienced the 2007 post-election violence as perpetrators or as victims. Such narratives hurt my emotions and affected the way I perceived the narrator.

Another bias was triggered by Sheng speakers' pejorative reference to Kenyans from the countryside as washamba, a term that had many contextual meanings in Nakuru and Mombasa. These included "country bumpkins," naïve, oldfashioned, backward and idiotic or dim-witted (some of these meanings will come up later in the dissertation). Since I was born, raised and schooled in the countryside, I felt like these speakers were attacking me and my people. Sometimes I felt defensive when speakers of Sheng claimed to be culturally sophisticated because rural societies, like where I was born, regarded the urban ones as culturally eroded for embracing western ways of life. However, I was able to overcome this cultural bias easily since I had also lived in major cities and could align with both sides of the urban-rural divide.

In order to strike a balance between these personal biases and a fair representation of my informants' views, I tried as much as possible to ask open-ended questions. I also audio-recorded our conversations rather than using pen and paper. In addition, I kept a personal journal, which was mainly a record of daily challenges and successes when interacting with my informants. I discuss this journal below.

\subsubsection{Personal journal}

This was a daily record of the challenges and successes that I experienced during fieldwork. However, it was not an easy or enjoyable exercise since many of the 
entries were about the frustrations I faced from my informants. Sometimes I would look at the journal and feel guilty because it looked like a record of wrongs. In such situations, I would feel like throwing it away in order to be able to let go of such frustrations. I thought that by clinging to my optimism, all would be well. Despite the intense struggle with my inner voices, I managed to make useful entries, some of which turned out to be very important when writing my dissertation. They reminded me about the mood I was in and potential biases when I made certain observations. Other entries helped me make sense of what was not said verbally, especially if I had noted down that the interview was conducted in a tense mood or the question/topic we were discussing was sensitive. Therefore, these entries sometimes provided consolation; others helped me check my behavior as I interacted with informants, while others helped me remain in touch with reality. Overall, the journal was a mirror of the journey that I walked during my stay in Nakuru and Mombasa.

\subsubsection{Leaving the field}

I concluded my research in Mombasa in early April and spent that month packing, clearing my apartment, and bidding goodbye to friends. I left for Nairobi at the beginning of May, 2012 and was housed again by my friend Shemeji. During this time, I processed my travel documents, including my U.S visa, before leaving for the U.S on June $4^{\text {th }} 2012$.

\subsubsection{Follow-up interviews via Facebook and E-mail}

Before leaving the field, I took email and Facebook contact information from some key informants, both teachers and students, from Nakuru and Mombasa. I did several follow-up conversations with them about some observations captured in my 
field notes which required clarification or elaboration. I have utilized some of them in developing arguments in the various dissertation chapters.

\subsubsection{Dissertation outline}

In Chapter Two, I focus on speakers' definition of Sheng. I challenge previous scholarly attempts to define it, arguing that they are inadequate since they attempt to fit Sheng into the existing linguistic criteria, which do not account for social factors such as speakers' attitudes and identities. These factors influence the way speakers perceive, employ and define Sheng. Instead, I argue that Sheng is socially defined; there is therefore need to seek how speakers themselves define it. The chapter demonstrates that not all young Kenyans speak Sheng, and neither are all language varieties associated with young Kenyans called Sheng. Instead, speakers employ different labels to refer to the language varieties they speak and in fluid ways. The label Sheng was the most commonly used in Nakuru while the situation in Mombasa was complex because there were additional insider-outsider distinctions among speakers. The majority identified with lugha ya mtaa, others with Sheng and the rest with both. The chapter ends with the argument that the Sheng phenomenon is not peculiar to Kenya. Instead, there are similar urban youth vernaculars in other major urban centers in post-colonial Africa, where speakers embrace them as alternatives to the officially sanctioned languages.

Chapter Three traces the development of the national language policy from the colonial period to date. The chapter seeks to establish the causes of the disconnect between these policies and peoples' language use in actual practice. I argue that Kenya has had an inconsistent language policy since the colonial period, because 
policy decisions are usually driven by political interests of the ruling class rather than the communicative needs of the masses. Thus, the state responds to the issues of language only when it feels threatened. The political nature of the policy formulation process in Kenya therefore explains why there have been many policy recommendations since the colonial period, but very of few of them have been implemented to date. This disconnect has partly been the reason why English, the language of the former colonial masters, has continued to enjoy its historical hegemonic status in Kenya.

Chapter Four examines the politics of national identity in relation to language in Kenya. I begin with a discussion of the problematic notion of "ethnic language,"arguing that it is a colonial construct that does not fit well in all cases. I then focus on the contested status of Standard Swahili, arguing that very few Kenyans use it in actual practice. Instead, many young Kenyans from different ethno-linguistic backgrounds prefer nonstandard varieties such as Sheng mainly in the upcountry and lugha ya mtaa in the case of Mombasa. With regard to adults, those from the upcountry speak contact varieties of Swahili which are closer to Sheng than Standard Swahili, while those from the coast speak local coastal varieties such as Kimvita, which is associated with Mombasa. Speakers of these language varieties in both Nakuru and Mombasa usually have a low regard for Standard Swahili. Therefore, I argue that there is a need for a review of the national language policy since the government has been investing in Standard Swahili as a national language, but the language does not have purchase among intended speakers. Further, I compare the status of Standard Swahili in Kenya and neighboring Tanzania, the only other country in the world where Standard Swahili has the status of a national and official language. 
I argue that the latter has succeeded in popularizing the language because of political and institutional support. However, I later argue that many people in both countries have a higher regard for English than Standard Swahili for various reasons that I discuss later in the chapter.

Chapter Five focuses on the paradoxical official language policy in Kenya. I argue that though both English and Standard Swahili are official languages, many Kenyans regard English as more official and important than Standard Swahili. As a result, these Kenyans are usually more worried about speaking English "correctly" than Standard Swahili. I attribute these perceptions to the colonial language ideology, which socially constructed foreign languages such as English and French as superior to African languages. This inequality was then naturalized through formal education which promotes "standard language ideology" (Lippi-Greene 2012) and related notion of "legitimate language" (Bourdieu 1977). I will discuss the two notions and question their viability in Kenya later in the chapter. The chapter ends with the argument that school authorities need to adopt alternative approaches of addressing students' use of nonstandard language varieties such as Sheng and lugha ya mtaa because the current measures are counterproductive.

Chapter Six discusses the motivations behind many young Kenyans' preference for the unauthorized varieties such as Sheng and lugha ya mtaa, while turning away from the officially sanctioned Standard Swahili and English. I argue that speakers embrace these nonstandard language varieties as alternatives to the other readily available languages. While school authorities argue that use of Sheng and lugha ya mtaa leads to poor performance in national exams, I demonstrate using my research findings that there are some speakers who employ the two language varieties 
productively such as during academic discussion groups and peer teaching. The chapter also discusses speakers' attitudes toward the officially sanctioned Standard Swahili and English, arguing that speakers exhibit resistance and conformity at the same time. I attribute this paradoxical demeanor to speakers' membership in different social groups which have competing values and interests. The chapter also challenges the common association of women with the use of standard language forms, while associating men with the nonstandard forms like Sheng. Instead, I demonstrate using research data that even women are active speakers and innovators of nonstandard forms too. Some women also use vulgar or taboo terms in their speech, further challenging the notion of femininity as socially constructed in the local cultural frameworks.

Chapter Seven focuses on the discourse of "shrubbing." Speakers used the term "shrubbing" basically to mean mispronouncing a word when speaking Swahili and English, due to various reasons such as mother tongue influence. Speakers attached multiple meanings to the act of shrubbing which were contextual. In my analysis, I use the term "shrubbing discourse" to mean the act of pointing out or highlighting that a speaker has shrubbed. I observed that shrubbing discourse occured among young Kenyans in Nakuru and Mombasa, but it is more conventionalized in Nakuru. Participants in both research sites required peers who spoke English and Swahili to do so without "errors." However, I argue that the discourse is paradoxical in various ways: despite participants' obsession with linguistic "correctness," they did not reward those who speak the two languages "correctly." Also, the discourse suggests both resistance and conformity to the officially sanctioned languages and associated identities. 
Finally, Chapter Eight is a recapitulation of the major research findings. It also summarizes the main arguments and conclusions made throughout the dissertation. The chapter ends with a brief discussion of the challenges I faced during fieldwork and suggests areas for further research on Sheng and related language varieties such as lugha ya mtaa. 


\title{
Chapter Two
}

\subsection{What is Sheng?}

\begin{abstract}
Sheng is a language of many hats. (Ms. Kamau 09/2011, casual conversation) ${ }^{12}$ Sheng is a moving target. (Ms. Githinji 09/2011, casual conversation) Sheng is about the question "are you one of us?" (Pastor Ng'ang'a 02/2012, casual conversation)

Sheng is a virus because it has no standard. (Mr. Maragwa 03/2011, casual conversation)
\end{abstract}

This chapter focuses on the contested definition of Sheng among speakers. Sheng is a term used by most Kenyans to describe a way of speaking that they associate with urban youth and matatu operators (Samper 2002). Previous researchers on Sheng report that it emerged in the early 1960s and 1970s among young Kenyans from diverse ethno-lingusitic backgrounds in the low-income, highly crowded neighborhoods of Nairobi, following the advent of the colonial economy (Abdul Aziz and Osinde 1997; Githinji 2006; Githiora 2002; Rudd 2008; Samper 2002; Spyropoulos 1987). Given the prevailing conditions, these scholars argue that young Kenyans needed a common medium of communication and a code for communicating in-group secrets. However, Sheng has spread to other major towns outside Nairobi and to the rural areas, where it has been embraced by other social groups such as young adults for different social functions (see Chapters Four, Six and Seven).

From a linguistic point of view, speakers of Sheng draw materials from various languages spoken in cosmopolitan neighborhoods such as English, Swahili and local "ethnic languages." They then fit these materials into the grammatical framework of the local variety of Swahili. ${ }^{13}$ In addition, Sheng speakers constantly

\footnotetext{
${ }^{12}$ All the quotations in this dissertation are in the original language used by the informants unless I indicate otherwise.

${ }^{13}$ The notion of "Swahili grammar" is itself problematic in the context of Kenya because there are many varieties of Swahili. See section 2.2.0 for more detail.
} 
revitalize it through ingenious manipulation of form and meaning to both existing and newly acquired linguistic materials. Speakers do so for different reasons discussed later in the dissertation. Consequently, Sheng exhibits overlapping characteristics of various linguistic categories such as pidgin, language mixing and dialect, but does not fit well into any of them. ${ }^{14}$ As a result, different scholars have defined and categorized Sheng in a range of ways, but I find these categorizations problematic because they do not take into account the role that social factors such as speakers' attitudes and their identities play in their definition of Sheng. Instead, I argue that the notion of style as a "social semiosis of distinctiveness" (Irvine 2001: 23) is the most appropriate analytical tool, because it recognizes the role of both linguistic and social factors in speakers' definitions of Sheng.

In the following section, I examine some of the existing scholarly definitions of Sheng, seeking to demonstrate that they are problematic. Also, I discuss my proposed notion of style and demonstrate, with the support of my research findings, that it is the most appropriate characterization of Sheng. In addition, I demonstrate that what speakers call Sheng is contextual and not all language varieties associated with young Kenyans are called Sheng. Instead, Nakuru and Mombasa have competing linguistic variants, which are embraced by speakers as tools for negotiating distinctiveness among other reasons. However, there are speakers who are able to weave in and out of various social categories for various reasons discussed further below. I start with previous scholarly attempts to define Sheng.

\footnotetext{
14 "A pidgin is a compromise language variety that arises in a new contact situation involving more than two linguistic groups that lack a shared language for communication. The contact groups develop a pidgin, with vocabulary drawn typically (though not always) from one of the languages in contact. The new pidgin's grammar doesn't come from any one language; instead, it is a kind of cross-language compromise of the grammars of the languages in contact" (Thomason 2001:159).
} 


\subsubsection{Previous scholarly definitions of Sheng}

One of the scholars who sought to define Sheng linguistically is Githiora (2002). He argues that even though Sheng exhibits pidgin-like characteristics such as lexical borrowing from the various languages in contact and reduction of form (e.g., reduction of the Swahili word sasa 'now' to the "heavy" syllable saa, p. 166), these features are insufficient to qualify Sheng as a pidgin. Also, he observes that the grammar of Sheng closely resembles that of Standard Swahili, hence he argues that Sheng should be described as an urban dialect of Swahili. However, Githiora's (2002) proposal is problematic because it fails to acknowledge that though Sheng and Swahili may resemble each other linguistically, many Kenyans (both speakers and non-speakers of Sheng) perceive Sheng and Swahili as distinct language varieties. Also, the notion of dialect does not capture the conscious role played by speakers in selectively borrowing and manipulating Sheng to enhance unintelligibility to outsiders and make it appropriate for serving other social functions. Further, Githiora's (2002) proposal has been faulted by other scholars. For example, Bosire (2006:190-191) argues that,

Unlike a regional dialect, Sheng is not localized in a particular geographical area and Sheng speakers span the many urban centers of Kenya unlike being congregated in Nairobi, the cradle. If we call it a sociolect, then we must define the social class that speaks Sheng.

In addition, Bosire (2006), Deen (2002) and Samper (2002) report that a Nairobi Swahili dialect exists, and is different from Sheng and Standard Swahili. (This distinction between Sheng and Nairobi Swahili is problematic, but I will return to it further below.) 
Samper (2002) and Bosire (2006) also sought to define Sheng linguistically. They argue that even though Sheng appears to be mainly code-switching between Swahili, English and Kenyan "ethnic languages,"defining or categorizing Sheng as code-switching fails to acknowledge that not all instances of code-switching in Kenya would be called Sheng. The two scholars also claim that such classification fails to capture the inherent cultural hybridity of Sheng and its speakers. Instead, they argue that Sheng would best be described as a hybrid language. According to Bosire (2006: 192), "a description of Sheng as a hybrid language captures the inherent duality of the product as both a linguistic and a cultural mixture as opposed to terms like mixed language, which may denote linguistic mixing without a reference to the intense cultural heterogeneity that is involved in such a project."

However, just like Githiora's (2002) notion of dialect, I find the notion of hybridity problematic. From a historical point of view, hybridity has colonial and racist connotations. In fact, Samper (2002: 35, citing Papastergiadis (1997), acknowledges that the notion was developed from $19^{\text {th }}$-century "scientific" racism (social Darwinism), which regarded some races as "superior" to others. In this context, cross-racial products were perceived as "impure", "degenerative", and "dangerous" to the "superior" race. From another point of view, even though hybridity recognizes non-linguistic factors such as manner of dressing that speakers employ to define Sheng, the notion does not capture why speakers do not agree on what they call Sheng. Specifically, hybridity as an analytical tool does not account for social factors such as speakers' attitudes, which would explain why when some Kenyans, especially those in Nairobi, are asked if they speak Sheng, they respond that 
they speak only "pure" Swahili or English. Interestingly, their response is in what the researchers identify immediately as Sheng (Githinji 2008).

Similarly, though Bosire (2008) and Samper (2002) claimed that Sheng and Nairobi Swahili are distinct language varieties, the ethnographic data contained in the various literatures about Sheng does not point to any clear-cut difference between them from a linguistic point of view. So, is Sheng really different from Nairobi Swahili? If so, how? To illustrate this problem, I first contrast Nairobi Swahili with Standard Swahili and then with Sheng.

\subsubsection{Sheng vs. Nairobi Swahili: how different are they?}

The term "Nairobi Swahili" is not widely used in Kenya. Indeed, there is some resistance to this term even though the type of Swahili most commonly used as a lingua franca in Nairobi differs from Standard Swahili in various ways. However, many Kenyans including linguists such as Deen (2002) acknowledge that the variety of Swahili spoken in Nairobi is not standard. ${ }^{15}$ According to Deen (2002: 17), Standard Swahili and Nairobi Swahili differ in their number of noun classes, grammatical agreements and the amount of lexical borrowing. ${ }^{16}$ With regard to noun classes, while Standard Swahili has 15, Nairobi Swahili has nine. For grammatical agreements, Standard Swahili has a more differentiated agreement morphology (e.g., subject and object agreements) than Nairobi Swahili. Specifically, apart from noun classes 1 and 2 where both language varieties have similar subject and object

\footnotetext{
${ }^{15}$ I avoid the term "non-Standard Swahili" because it would be confusing in the context of Kenya, where there are varieties that originated in situations of contact between speakers of other languages which could be described as "nonstandard." Also, there are coastal varieties such as Kimvita which have been spoken as mother tongues for centuries but also differ from what is called "standard."

${ }^{16}$ The numbers used stand for the Noun Classes. According to the traditional Bantu numbering system (Bleek 1869), singular and plural forms of nouns are given different, adjacent class numbers. For example ' 8 ' indicates that the noun in question carries the plural prefix $v i$ - which corresponds to the singular prefix $k i$ - of class 7 (Contini-Morava 2000).
} 
agreements, Standard Swahili has a different agreement form for each noun class. Nairobi Swahili marks the rest of its noun agreements with $i$ - in singular and $z i$ - in plural. The last difference is that Nairobi Swahili borrows a lot more than Standard Swahili especially from English, Kikuyu and Dholuo, which are the three other main languages spoken in Nairobi. The example in table (1) below illustrates some of these differences.

Table (1): A Comparison of Standard Swahili and Nairobi Swahili

\begin{tabular}{|l|l|l|}
\hline & Standard Swahili & Nairobi Swahili \\
\hline (i) & Vi-tabu vi - me - anguk-a & Vi-tabu zi -me - anguk-a \\
& 8-book SApl8-pr.prf.-fall-IND ${ }^{17}$ & 8-book SApl-pr.prf.-fall-IND \\
& 'The books have fallen' & 'The books have fallen' \\
\hline
\end{tabular}

(Adapted from Deen 2002: 22)

While it is possible to differentiate between standard and Nairobi Swahili linguistically as demonstrated in Table (1) above, the example in Table (2) below shows that it is hard to differentiate between Nairobi Swahili and Sheng because of the similarity in their grammatical agreements. The example in the left column was identified as "Nairobi Swahili" by Deen (2002: 22) while the example on the right was identified as Sheng in the Sheng Online Bible. ${ }^{18}$ The two examples do not differ from a grammatical point of view but they do in vocabulary. However, this in itself is not a reliable basis for distinguishing them, because both Nairobi Swahili and Sheng borrow extensively from other languages in contact.

\footnotetext{
17 The abbreviates used below the examples are glosses as used by Deen (2002) for: "SApl"- Subject Agreement plural, "pr.pfr"-present perfect tense, "fv"- final vowel, "ftm" future tense marker, "dem" demonstrative.

${ }^{18}$ Sheng online Bible is a blog run by a group of young Kenyans who call themselves Wasee wa Sir Godi or Mavijanaa wa J.C 'People of God or Youth for Jesus Christ in Sheng'. The blog says that the main goal of these young Kenyans is to translate the Bible from English to Sheng in order to reach more youth spiritually, especially those who speak Sheng. The link to the Sheng online bible is: http://shengilia.blogspot.com/2009/06/matthew-24.html
} 
Table (2): A Comparison of Nairobi Swahili and Sheng

\begin{tabular}{|l|l|l|}
\hline & Nairobi Swahili & Sheng \\
\hline (i) & Vi-tabu $\mathbf{z i}-$ me - anguk - a & Vi-tu zi -ta - go down \\
& 8-book SAp19-pr.prf.-fall-fv & 8-things SAp19-ftm. Go down \\
& The books have fallen & Things will this happen \\
\hline
\end{tabular}

Several other researchers such as Abdul-Aziz and Osinde (1997) and Rudd (2008) who sought to define Sheng fell into the same trap of fitting it into the available linguistic criteria, rather than adopting multiple approaches or seeking to know how speakers of Sheng define it. In contrast, my dissertation introduces a new point of departure in the definition of Sheng. It employs a theoretical approach that recognizes that social factors such as speakers' attitudes, their identities and language ideology influence how they perceive, define and employ Sheng in their conversations. ${ }^{19}$ Also, my dissertation sheds light on how Sheng is defined, used and perceived in different settings. I draw data from speakers living in previously unexplored geographic and social contexts outside Nairobi, the perceived origin of Sheng. My approach therefore acknowledges that not all youth in Kenya speak Sheng on the one hand, and all youth languages in Kenya are not called Sheng on the other. Instead, speakers in different geographical and social environments have their own categories that they use to define the language variety that they speak. In order to account for speakers' variations in what they regard as Sheng in relation to the language variety they speak, I argue that Irvine's (2001: 23) notion of styleis the most appropriate analytical tool. I discuss this notion in detail here below.

\footnotetext{
${ }^{19}$ Language ideology has been defined differently by different scholars, but Silverstein (1979: 193) argues that it can basically be understood as "sets of beliefs about language articulated by users as a rationalization or justification of perceived language structure and use." According to Silverstein (1979), this notion involves people's beliefs or socio-political constructs about the relationship between languages, their speakers and the social world. These beliefs are usually subjective, hence they can affect how speakers interpret linguistic practice.
} 


\title{
2.3.0. The notion of "Style"
}

The meaning of "style" is broad and varies across contexts; hence different scholars have defined and used it differently. In the case of Sheng, I find Irvine's (2001) conceptualization of style as a "social semiosis of distinctiveness" the most appropriate analytical tool. By this, Irvine (2001: 31) refers to the various linguistic and nonlinguistic ways that "individuals navigate among available varieties and try to perform a coherent representation of self - a self that may in turn be subdivided into a differentiated system of aspects-of-self." However, she cautions that even though style may characterize an individual, there has to be a social framework of witnesses who pay attention and provide social evaluation. More specifically, Irvine (2001: 2324) asserts that:

\begin{abstract}
Styles in speaking involve the way speakers, as agents in social (and sociolinguistic) space, negotiate their positions and goals within a system of distinctions and possibilities. Their acts of speaking are ideologically mediated since those acts necessarily involve the speaker's understanding of salient social groups, activities and practices, including forms of talk. Such understandings incorporate evaluations and are weighted by the speaker's social position and interest. They are also affected by the differences in speaker's access to relevant practices. Social acts, including acts of speaking, are informed by an ideologized system of representations, and no matter how instrumental they may be to some particular social goal, they also participate in the work of representation.
\end{abstract}

Irvine's conceptualization of style builds upon Peirce's (1931) theory of iconic signs that explains the underlying meanings of the links between objects (linguistic signs in this case) and their referents (social meanings). According to Peirce (1931; see also Ahearn 2012), a sign is said to be iconic if the signal involved resembles the meaning that is being signaled or expressed. In the case of distinctiveness, if the meaning being signaled is social difference, then linguistic difference can serve as an icon of social difference. Building on this knowledge, Irvine (2001) looks at the use of a language variety not as an object in itself but as a continuous semiotic process of 
differentiation. As such, she extends the linguistic sign-referent relationship to include "the relationships between the sign and its conditions of production, i.e., the speakers or the activities or scenes in which it is characteristically uttered" (Irvine 2001: 34).

As an analytical tool, Irvine's notion of "style" is useful in understanding Sheng because style manifests itself in every aspect of language and extends to associated aesthetics such as mode of dressing, hairstyle, speakers' language ideology, their principles of differentiation and the social meanings attached to their differential use of language. Also, style recognizes the multiple levels of meanings of individual variables and their fluidity, and that the links between those variables and their social meanings are ideologically mediated (Coupland 2007; Eckert 2008; Irvine 2001).

Due to the wide range of phenomena assignable to the notion of style (which has the potential to create analytical chaos), and in order to explain how speakers selectively and creatively utilize available semiotic resources to make meaning, Irvine's (2001), style employs a three-pronged model comprised of "iconization", "recursivity" and "erasure." According to Irvine (2001:33), "iconization entails the attribution of cause and necessity to a connection between linguistic behaviors and social categories - of people or activities." For example, Sheng is often associated with low-status groups such as manambas, and this association contributes to both its rejection by school authorities and its embrace by young people as a language of resistance to authority. Ethnic languages in contrast are associated with illiteracy and stereotypes about particular ethnic groups (Samper 2002). For recursivity, Irvine (2001: 33) says that it "involves the projection of an opposition, salient at one level of relationship, onto some other level..." For example, the perception of Sheng as a 
lingua franca with no particular ethnic associations, in contrast with "ethnic languages," manifests itself both at the level of language choice and within the vocabulary of Sheng, which is drawn from several different languages (Githinji 2006; Ogechi 2005; Samper 2002). Lastly, Irvine (2001: 331) defines erasure as the "process in which an ideology simplifies the sociolinguistic field; attending to one dimension of sociolinguistic phenomena (or persons or activities) while rendering others invisible." For example, the common association of Sheng with males and students (Githiora 2002; Samper 2002) renders the use of Sheng among females and teachers invisible.

Unlike the previous scholars who employed fixed linguistic categories such as dialect, pidgin and mixed language to define Sheng, my preferred notion of style acknowledges among other factors; the fluidity and variation in Sheng, speakers' contradictions in their definition of Sheng and nonlinguistic factors such as speakers' attitudes and their identities. So, how is Sheng a "social semiosis of distinctiveness?"

\subsubsection{Sheng as style}

Sheng, like style, means different things to different people in different contexts. As such, individual speakers and social groups in both Nakuru and Mombasa had different understandings of what Sheng is and their definitions were usually contextual. Also, their language attitudes were sometimes contradictory. For example, teachers in Kenya have multiple identities with competing values and interests; hence their attitudes toward Sheng are usually contextual. In Nakuru, for example, Mrs. Kiboko who taught Home Science, and was also the head of guidance, expressed ambivalence towards Sheng. In one context, she criticized it arguing that it 
was detrimental to the students' use of Standard Swahili and English (Mrs. Kiboko 11/2011, interview). She claimed that many students spoke Sheng most of the time, both within and outside the school, hence they found it difficult to switch to English or Standard Swahili when required to do so. She elaborated that students were so comfortable with Sheng that when they came for guidance, what they said in Standard Swahili or English was usually overly direct translation of what was in their minds. As a result, Mrs. Kiboko claimed that students end up giving responses that she did not understand or that she found "crazy."

Consequently, she said that she spent a significant amount of time during counseling, teaching her clients "good" English. For example, she told me that it was common for students who went for counseling to ask the teacher to "borrow water," meaning a request for some water. Teachers criticized this as inappropriate use of the English verb borrow, since the student will consume the water rather than giving it back. This seems to be a mother-tongue influence, a direct translation of the respectful expression borrow that might be used in various Kenyan languages, but the teachers attributed the "error" to Sheng influence. In the same interview, Mrs. Kiboko claimed that students who spoke Sheng performed poorly in exams, since their embrace of Sheng had interfered with their reading culture. She elaborated that the lack of a reading culture was why these students could not communicate effectively in either Standard Swahili or English. Since excelling in national exams is associated with good career prospects in Kenya, Mrs. Kiboko found students' use of Sheng socially and geographically limiting. This is because students would be required to speak English and sometimes Standard Swahili when applying for jobs both within and outside Kenya. 
However, despite Mrs. Kiboko’s high regard for the use of English and Standard Swahili, she evaluated Sheng positively in some other contexts. For instance, she sometimes allowed some of her student clients who could not express themselves adequately in either Standard Swahili or English to switch to a language in which they were comfortable. In such situations, if a client used Sheng words or expressions that Mrs. Kiboko did not comprehend, she asked them to provide meanings in a language that both she and the client could understand. I present these sentiments in the following extract of an interview segment that was conducted in English:

Mrs. Kiboko: For me personally, I do speak a lot in English but like they say, you have to be sensitive, and in counseling you listen with more than just your ears. Sometimes you listen with your heart and you see what this child is not saying and so sometimes there are those who will get stuck in language and I tell them, Ok if you are comfortable, can you just switch as long as you tell me what you want.

\section{MW: Do you specify?}

Mrs. Kiboko: I tell them, "Can you speak in Kiswahili?"

MW: Ooh Ok, Ok

Mrs. Kiboko: And they say, "Yes teacher." And if you are more comfortable in that, it is ok, you speak in that. And they go ahead and do that.

\section{MW: And what Kiswahili do they speak?}

Mrs. Kiboko: They speak good Swahili when they come to my office because they know I am not very good at Sheng [both laugh]. So they know they might lose me. And if they do, I do find them speaking in Sheng, I'll ask for a clarification.

(Mrs. Kiboko 11/2011, interview)

While this language use strategy helped Mrs. Kiboko to reduce social distance and upgrade her own Sheng, it also suggests that communication was not really the main problem in these encounters. Instead, it was Mrs. Kiboko 's language ideology which prescribed ability to communicate in certain ways such as use of English rather than other languages, as appropriate for certain social contexts. In two other related contexts, Mrs. Kiboko confessed that even though she did not know much Sheng, she 
sometimes useds a few words and expressions during counseling, or when teaching in order to enhance students' attentiveness among other reasons. For example, when I asked her whether she spoke Sheng when interacting with students, she responded that,

Ok, like if am doing my own small in-house thing and am talking with these children [students], I may occasionally throw in a word or two, yah, to break the ice, to have them relax, so that we listen to one another and to also let them know am with them. (Mrs. Kiboko 11/2011, interview)

At the end of the interview, Mrs. Kiboko expressed the view that authorities should not negate or fight Sheng. Instead, Sheng should be left to grow on its own provided that it does not grow at the detriment of Standard Swahili and English.

There were also students in Nakuru and Mombasa who expressed ambivalent views of Sheng like Mrs. Kiboko, because they had multiple and competing identities. These were mainly those with leadership positions or who performed well academically, because they had to negotiate a balance between pleasing the school administration on the one hand, and associating with peers on the other. For example, I asked students in a focus group in Nakuru to comment about the future of Sheng. Among them was the school captain/head boy, Mwelusi, who responded by discussing his perceptions toward Sheng in relation to English and Standard Swahili (Form Four focus group 3, 11/2011--Nakuru). While his language attitudes seemed contradictory from an outsider's point of view, I argue that he is performing what Samper (2002) calls "walking the borderline," i.e., balancing between competing identities.

Mwelusi started by saying that he did not like speaking English because of its colonial legacy. Specifically, he claimed that former colonial masters spoke English 
when planning how to take over his grandfather's land, since they knew that he did not understand the language. For these reasons, Mwelusi said that colonialists were of no good to Kenyans; hence Kenyans would want to forget them and move on.

However, he wondered how that would be possible when Kenyans were still using the same colonial language for communication. Despite criticizing former colonial masters, he claimed that there would be no formal schools in Kenya were it not for them.

With regard to Standard Swahili, Mwelusi said that it is a very difficult language at the level of vocabulary and pronunciation. He compared it with English, arguing that unlike the latter which has silent sounds, each and every sound in Standard Swahili has to be pronounced, which is a big challenge to many speakers. Consequently, he said that speakers resort to Sheng, claiming that it provides them with alternative lexical items, which have sounds that are easier to pronounce. Therefore, Mwelusi claimed that though Sheng is already widespread, it would continue spreading in future and even at a faster rate (Form Four focus group 3, 11/2011--Nakuru).This claim suggests that his response to my question was that Sheng has a bright future in Kenya. However, Mwelusi later claimed that Sheng should be abolished since it interfered with students' mastery of Standard Swahili and English further leading to poor performance in their exams. He associated Sheng with students who often broke school rules and who did not take education seriously. These students were called Marui within the student community. ${ }^{20}$

\footnotetext{
${ }^{20}$ Marui is a label used among students in Nakuru to mean peers who have lost academic focus and who often break school rules. The term is derived by prefixing the Swahli plural marker $m a$ - to the trunctaed root of the English verb "ruin."
} 
In Mombasa, student monitors exhibited similar ambivalent attitudes toward Sheng. For example, one of the senior male prefecrts, Myumba, claimed in an individual interview that he was a bona fide speaker of Sheng, but later associated it with those who performed poorly academically. He told me that such students were called "Choka mbaya" among peers (Myumba 03/2012, interview). ${ }^{21}$ Interestingly, I later learned from some teachers who saw me walking and conversing with Myumba that he was an academic non-performer, and one of the students whom teachers were watching closely because of discipline problems. So, why did the various informants like Myumba in Mombasa and Mwelusi in Nakuru express ambivalent attitudes toward Sheng?

I argue that uses and perceptions of Sheng are ideologically mediated. Student Mwelusi's and Myumba's positive attitudes toward Sheng indicated their desire for solidarity with other speakers of Sheng. Their association of Sheng with Marui and Choka mbaya on the other hand demonstrated that they recognized other people's (especially teachers') ideology about Sheng. Similarly, they were conscious about their identities as senior prefects, which require them to conform to the school's language policy. Possibly, since Mwelusi and Myumba knew that I used to interact with teachers and the school administration, they enacted conformant social personae in our interactions, to impress me. However, Myumba's claim that he was a bona fide speaker of Sheng demonstrates that he recognized the oppositional language ideology common among young people that it is desirable to speak and associate with Sheng. So, while the various stances (Jaffe 2009) toward Sheng may seem contradictory to an outsider, speakers do not perceive them that way. Instead, they regard them as

\footnotetext{
${ }^{21}$ Choka mbaya is a label used by students in Mombasa to refer to the category of students who are generally negative toward school.
} 
different parts of the same "self." In other words, speakers are conscious of these multiple identities and they perform them as survival tactics.

Given that social factors play a significant role in speakers' use and perceptions of Sheng, there are speakers who, as mentioned earlier, deny that they speak Sheng but their response is in what the researchers identify as Sheng. In Nakuru for example, I asked one teacher whom I will call Mrs. Kizito whether she spoke Sheng, but she denied this and attributed the use of Sheng to young Kenyans, especially the "dodgy fellows in the streets and ghetto" (Mrs. Kizito 10/2011, casual conversation).

However, I observed on several occasions that Mrs. Kizito often employed linguistic constructions that some speakers would identify as Sheng. In one prayer meeting involving students, teachers and parents, she called upon the congregation to be more active in singing and dancing to warm up for the event. She asked:

1. Si tuko na imani tutabarikiwa? Tumdansie Mungu basi! Hii si funeral! Tumshangilie. Na tutadance. Vyenye utaingia mbele ya bwana ni wewe utadetermine.
1. Don't we have faith that we will be blessed? Then, let's dance for the Lord. This is not a funeral! Let's praise and celebrate Him. And we will dance. How you will get to heaven, you are the one to determine.

(Mrs. Kizito 10/2011, direct observation)

From a linguistic point of view, the passage above appears to be Standard Swahili with the addition of a few English words (dance, funeral, determine). However later, I presented a written transcript to Mrs. Kizito and explained that I recorded those words when she was addressing the congregation. I asked her to comment about the language(s) used and she described it as Sheng. She further explained that it has become necessary for adults nowadays to speak Sheng in order to reach the youth. The two conversations with Mrs. Kizito indicate that speakers' identity and language ideology play a significant role in their definition and 
employment of Sheng. Possibly, Mrs. Kizito first denied that she spoke Sheng since associating herself with it would put her identity as a teacher at stake. That is, the school administration expects teachers to promote the official language policy.

From another perspective, claiming that she spoke Sheng in order to reach the youth suggests that she was aware of the language ideology that Sheng is more appealing to the youth than Standard Swahili or English. Such social factors cannot be captured using the existing linguistic criteria such as dialect, hybridity, slang and language mixing. Similarly, these linguistic categories do not explain speakers' lack of consensus about what Sheng is. In this vein, previous scholarly attempts to define Sheng do not acknowledge that it is a contested sociolinguistic phenomenon among speakers. Instead, I argue that the broad range in speakers' understandings of Sheng and contributing social factors would best be captured using Irvine's (2001) notion of style because it manifests itself in every aspect of language. Next, I discuss how various categories of speakers in Nakuru and Mombasa defined Sheng, and demonstrate that Sheng is a contested sociolinguistic phenomenon for various reasons.

\subsubsection{Speakers' definitions of Sheng}

My field observations indicate that speakers' definitions of Sheng in both Nakuru and Mombasa were very diverse. That is, different categories of speakers emphasized different aspects in their definitions such as what languages must be there for a given linguistic construction to be called Sheng. There were also individual speakers with multiple definitions. For example, I asked a student, Mpenda, in a casual conversation in Nakuru to comment about the language(s) that students in her 
school spoke most of the time. She responded that they spoke mamixture, ${ }^{22}$ which she defined as a mixture of English, Swahili and Sheng (Mpenda 9/2011, casual conversation). I then asked Mpenda to describe what she meant by the term Sheng. She responded that, it is a language spoken by the youth and gave the following words as examples of Sheng: mamixture, bwaku ${ }^{23}$ and beshte. ${ }^{24}$ A critical look at Mpenda's response reveals that she had different conceptualizations of Sheng namely: 1) Sheng as single lexical items; 2) Sheng as a mixture of several language varieties; 3) Sheng as a distinct language variety from Swahili and English; and 4) Sheng is a language variety associated with particular social groups such as youth. I build upon these four definitions but drawing examples from other categories of speakers here below.

\subsubsection{Definition of Sheng at the lexical level}

I asked students in a focus group in Nakuru about what they called Sheng. One of them, Bakari, responded that Sheng is "unique" vocabulary from various languages such as mother tongue (ethnic languages), or coined ones that are introduced to Swahili or English conversations (Form Three focus group, 11/2011--Nakuru). He mentioned the word kadete, as an example of Sheng that has widely been embraced by peers. According to Bakari, kadete was coined by one of his close friends to refer to the act of talking too much. In the same focus group, Bakari told me that one of the participants, Wandera, was a well-known speaker of Sheng and this prompted me to ask him to say something in Sheng. He responded by mentioning individual lexical items as reflected in the following extract:

\footnotetext{
${ }^{22}$ The word mamixture is generated by prefixing Swahili plural marker $m a$ - to the borrowed English word "mixture."

${ }^{23}$ Bwaku is generated by reversing the syllables of the Swahili word kubwa 'big'.

${ }^{24}$ One of the informants told me that the term beshte means friend in Sheng. Some informants used the term 'bestie', which is teenage English for best friend.
} 
1. Bakari: Huyu ndiye nilikuwa nakwambia [...], amebombea katika Sheng.

2. MW: Eeh, umebombea?

3. Wandera: Eeeh

4. MW: Hebu tuongeleshe Sheng kidogo. Pick tu kitu utwambie.

5. Wandera: Aah kitu kama cheda. Cheda, hiyo ni doo; wengine wajua ni doo, wengine wajua ni pesa, wengine wajua ni gwara, yaani vitu mingi hivo.

6. MW: Na how do you feel ukiambiwa na wengine eti wewe ndiye umebombea kwa Sheng? How does it feel?

7. Wandera: Eeeh, si unabambabika tu.
1. Bakari: This is the one I was telling you [...] He is a champion of Sheng.

2. MW: Eeh, so you are good in Sheng?

3. Wandera: Eeh

4. MW: Please speak a little Sheng to us. Just pick something and tell us about it.

5. Wandera: Aah something like cheda; that is money. Some people call it doo while others call it gwara. So, things like those.

6. MW: Now, how do you feel when others regard you as a champion of Sheng? How does it feel?

7. Wandera: Eeeh it really makes me feel good!

(Form Three focus group, 10/2011--Nakuru)

In Mombasa, I also observed that there were speakers who defined Sheng at the lexical level. For instance, I asked students in a focus group whether there was a difference between what speakers in Mombasa called lugha ya mtaa and Sheng, which they associated with Nairobi. One of the students, Zawadi, responded that the two language varieties were different, and gave examples of individual lexical items as shown in the following extract:

1. Mimi nafikiri ni tofauti, kwa sababu ukiangalia Sheng sana huwa Kizungu kama ambacho kimeteguliwateguliwa [...]. Wamekitohoa kidogo kama grandie sasa ndio iyo grandparents, kama rodi $n i$ barabara, kama maparoo ni parents, masistie ni sisters, mabroo ni mabrothers.
1. I think they are different because if you look at Sheng critically, it is usually English that has been manipulated [...] They [speakers] have Swahilized English words like grandie for grandparents, rodi for road, maparoo for parents, masistie for sisters and mabroo for brothers.

Form Two focus group 2, 03/2012--Mombasa)

The definitions above agree with the existing literature such as Githinji (2006), who report that the basic definition of Sheng is at the lexical level. Another category of speakers like student Mpenda mentioned further above defined Sheng as a mixture of various languages. However, they differed on the combination of language varieties 
that make up Sheng, indicating that Sheng is a contested social fabric among

speakers. I discuss a few examples here below.

\subsubsection{Sheng as a mixture of various languages}

I presented a poster that had two expressions written in bold to a teacher in

Nakuru, Mrs. Mob, and asked her to comment about the language(s) in which they

were written and their meanings. The two expressions were Chips Funga and Wacha

Mpango wa Kando. Mrs. Mob's response was as follows:

1. MW: Here I have some materials mwalimu, mmh, I got them from the guidance and counseling notice board. I just want you to read these, these two road signs. This one and this one.

2. Mrs. Mob: Wacha mpango wa kando, chips...what?

3. MW: Chips funga

4. Mrs. Mob: Eeh

5. MW: Eeh? What, in what language are those words?

6. Mrs. Mob:[...] This one is not standard Kiswahili. Chips funga .

7. MW: That one is not Standard Swahili?

8. Mrs. Mob: Ehe, hiyo ni Sheng,

9. MW: Ok, [laughter]. And the other one, wacha mpango wa kando?

10. Mrs. Mob: Sasa, iyo ni ya mtaa [laughter]. But it is giving [...] It is giving the message

11. MW: Ehe

12. Mrs. Mob: Eeh. It is giving the message

13. MW: Why have we said Chips funga is Sheng and Wacha Mpango wa Kando ni lugha ya mtaa?

14. Mrs. Mob: Wacha Mpango wa Kando. There is no, this one has English, Kiswahili. This is one is Kiswahili, lakini si ile standard

15. MW: Eeh

16. All: Laugh.
1. MW: Teacher, here I have some materials that I got from the guidance and counseling notice board. I just want you to read these, these two road signs. This one and this one.

2. Mrs. Mob: Stop side plans, chips ... what?

3. MW: Wrap Chips

4. Mrs. Mob: Eeh

5. MW: Eeh? In what language are those words?

6. Mrs. Mob: [...] This one, is not standard Kiswahili "Wrap chips"

7. MW: That one is not Standard Swahili?

8. Mrs. Mob: Ehe, that is Sheng,

9. MW: Ok, [laughter]. And the other one, "stop side plans"?

10. Mrs. Mob: Now, that is of the street [laughter]. But it is giving $[\ldots]$ It is giving the message

11. MW: Ehe

12. Mrs. Mob: Eeh. It is giving the message

13. MW: Why have we said "Chips Funga" is Sheng and "Wacha Mpango wa Kando" is street language?

14. Mrs. Mob: Wacha Mpango wa Kando. There is no, this one has English, Kiswahili. This is one is Kiswahili, but not standard.

15. MW: Eeh

16. All: Laugh.

(Mrs. Mob 10/2011, interview)

Mrs. Mob categorized Chips Funga as Sheng and Wacha Mpango wa Kando as

Kiswahili ya Mtaa 'street Swahili'. She categorized Chips Funga as Sheng, saying

that the expression draws the word Chips 'French fries' from English and Funga

'wrap' from Swahili. This characterization of Sheng as a mixture of English and 
Swahili is consistent with the existing literature. For example, Mazrui (1995) observes that Sheng is basically "Swahili+English," hence the acronym "Sheng" (also see Githinji 2006: 5; Migunda-Attyang 2007). This definition is also found in several blogs and online forums run by young Kenyans. ${ }^{25}$

Despite the fact that the above definition of Sheng was common in both Nakuru and Mombasa, some speakers contested that mere use of English and Swahili in a single conversation does not necessarily amount to Sheng. Instead, they told me that for such a mixture to be called Sheng, it must incorporate coined elements, draw from ethnic languages, or use expressions that have undergone ingenious linguistic alterations such as syllabic reversal. For example, I asked students in a mixed-gender focus group in Nakuru to comment on the language(s) that most students used in writing Autobio, apersonalized book that highlights memorable moments with friends. One of them, Faridzeh, responded that it depended on the individual writer. She then elaborated that the majority wrote in English, some used Swahili, others Sheng and the rest mixed Swahili and English. I then asked her whether there was a difference between Sheng and that mixture of English and Swahili. She said that they were different and described them as follows:

1. Faridzeh: As in, Tichaa tulikwambia, as in, English na Kiswahili ni pure English na pure Kiswahili. But Sheng utamix the languages. Utamix that English, that Kiswahili and then some other borrowed languages from other things and may be venye umetwist hiyo Kiswahili yako ama English yako.

2. MW: Ooh, so nikiongea like Kiswahili sanifu unaona kama venye mimi huwa nachanganya, sasa siwezi dai ati naongea Sheng?

3. Faridzeh: Sasa hiyo umesema dai ni Sheng

4. MW: Aah, si dai ni la Kiswahili?
1. Faridzeh: As in, we told you teacher, as in, English and Kiswahili are pure English and pure Kiswahili. But for Sheng, you mix the languages. You mix that English, that Kiswahili and then some other borrowed languages from other things and may be some that you have twisted your Kiswahili or English.

2. MW: Ooh, so If I speak let's say Standard Swahili. You see like the way I usually mix, now I cannot claim that I am speaking Sheng?

3. Faridzeh: Now that dai that you have said is Sheng.

4. MW: Aah, isn't dai a Swahili word?

${ }^{25}$ http://www.kenyanlist.com/kls-listing-show.php?id=38090 
5. Faridzeh: Inatumika kama Sheng bado.

6. $\mathrm{MW}: \boldsymbol{O k}$.

5. Faridzeh: It is still used as Sheng.

6. MW: Ok.

Form Two mixed-gender focus group 10/2011--Nakuru)

While the various examples given above define Sheng as a mixture of various languages, speakers in both Mombasa and Nakuru told me that a mixture of ethnic languages without elements of Swahili and/or English does not amount to Sheng. Instead, they dismissed it as individual speaker's "own thing" or "stuff," since no one would understand what the speaker is saying. They said that such a mixture would only become Sheng if it was swahilized, i.e., fitted into the overarching grammatical framework of Swahili. These sentiments are encapsulated in the following extract:

1. MW: Is there a mixture of languages that we cannot call Sheng?

2. Otis: Eeeh,

3. MW: Kwa mfano?

4. Otis: Kwa sababu saa huwezi mix lugha za mama na uiite Sheng saana. Kwa mfano mimi nikimix saa lugha kwa mfano Kiluhya na Kijaluo, hapo hapo hivo hakuna Sheng.

5. MW: Hiyo ni nini?

6. Otis: Hapo, hapo, hivo, aaah hizo tu ni vitu tu zako.

7. All [laugh]

8. Odusoh: Halafu unajua Sheng, unajua Sheng sasa, mimi naweza sema Sheng sana sana imetoa kwa Kiswahili. Ndio maana kila kitu imekuwa Sheng imeswahilishwa kutoka kwa lugha tofauti. Unaona?

9. MW: Mmh.
1. MW: Is there a mixture of languages that we cannot call Sheng?

2. Otis: Eeeh,

3. MW: For example?

4. Otis: Because now you cannot mix ethnic languages and call them Sheng. For example, if I mix languages such as Luhya and Dholuo, right there, there is no Sheng.

5. MW: What is that?

6. Otis: There, there, aaah those are just your own stuff.

7. All [laugh]

8. Odusoh: Then you know Sheng, you now Sheng now, I can say Sheng draws a lot from Swahili. That is why everything that has become Sheng has been Swahilized from different languages. You see?

9. MW: Mmh.

(Form Four focus group 4, 11/2011--Nakuru)

Despite the strong emphasis that speakers put on the role of Swahili in the production of Sheng, some speakers were categorical that Sheng and Swahili are two different language varieties. Others claimed that Sheng is a nonstandard variety of Swahili. But why would some speakers claim that Sheng is a variety of Swahili, while others perceived it as a distinct language variety? Along the same vein, what does it mean for a linguistic variety to be called a language? I engage these questions here below, starting with the 
claims that Swahili and Sheng are distinct language varieties. Speakers in this category based their arguments on whether the language in question has a dictionary and its own, stable grammar.

\subsubsection{Definition of Sheng on the basis of dictionary or lack of it}

Student Odusoh from the focus group introduced above claimed that though words drawn from ethnic languages are Swahilized to make them Sheng, they cannot be regarded as Swahili since they have not been added to the Swahili dictionary. He gave an example of the Kikuyu word tenee, 'early or long time ago', which has taken the place of the Swahili word zamani in peoples' conversations. He, however, claime that tenee was still Sheng since it has not been accepted (added to the Swahili dictionary). These claims are reflected in the following extract:

1. Odusoh: Saa kama may be zamani sasa watu wameachanga kusema ati "mimi nilikuwa huko zamani." Sasa wanasema "mimi nilikuwa huko tenee." Sasa kaa tenee unacheki, haiko kwa kamusi lakini sasa inatajwa kaa Kiswahili.

2. MW: Mmh, so unapotumia neno tenee wakati huo haulitumii ati ni la Kikuyu?

3. Odusoh: Eeh.

4. MW: Limeshaingia kwa Kiswahili?

5. Odusoh: Eeh, lakini kwa vile haliko kwa kamusi, ndio linaitwa Sheng sababu halijakubalika. Unaona? May be hata msee anaweza akasema, yaani unaweza ukabadilisha, yaani kuswahilisha sana sana lakini haijakubalika. Unaona?
1. Odusoh: Now like in the case of the word Zamani ['early or in the past'], people have stopped saying, "I was there early or in the past." Now they say "I was there tenee." Now you see like tenee is not in the Swahili dictionary but it is pronounced as Kiswahili.

2. MW: Mmh, so when you use the word tenee you don't use it as a Kikuyu word?

3. Odusoh: Eeh.

4. MW: The word has already entered into Swahili?

5. Odusoh: Eeh, but since it is not in the Swahili dictionary, that is why it is called Sheng. Because it has not been accepted. You see? May be even a person can say, that is, you can change, you can Swahilize but it has not been accepted. You see?

(Form Four focus group 4, 11/2011--Nakuru)

The presence of a dictionary gives a language some legitimacy because dominant institutions such as schools value the written language more than the spoken. Also, the value is placed on conventionalization and homogeneity in 
conformity with "standard language ideology" (Lippi-Green 2012, see further discussion in the following section). The case of Sheng is interesting because some speakers claimed that there existed a dictionary of Sheng while others claimed that there was not. I observed that those who claimed that Sheng had a dictionary perceived it positively and saw a bright future for Sheng in Kenya (see Chapter Four). Those who claimed that Sheng did not have a dictionary were opposed to it. They claimed that Sheng is highly volatile; hence it would be difficult to produce a reliable dictionary. For example, one teacher, $\mathrm{Mr}$. Maragwa, from Nakuru claimed that "Sheng is a virus," which he elaborated as follows:

\begin{abstract}
Sheng is a virus because it has no standard. When you have no standards, things have no store. By the end of the day, you have nothing. This is because standards help us maintain what we have. The Sheng we have is a street language that has no custodian. Because of the lack of standards, the generation we have today will lack a language of communication because it is only a language of the day/today not for tomorrow. Sheng is a language that cannot be controlled. Sheng is wild because one word in Sheng has many meanings. As such, Sheng lacks limits. Sheng is like a computer virus which destroys all the stored information in a computer. It destroys the standard language. (Mr. Maragwa 03/2011, casual conversation)
\end{abstract}

For these reasons, those opposed to Sheng like Mr. Maragwa claimed that it cannot be used as a medium of classroom instruction or as national language in Kenya (see Chapter Five).

\title{
2.3.2.4. Definition of Sheng on the basis of grammar or lack of it
}

Another category of speakers told me that Sheng is different from Swahili since Sheng does not have a grammar of its own. For example, in student Mwelusi's focus group in Nakuru, introduced earlier, I sought to know the meaning of the expression "Kiswahili kimemixiwa na Sheng”, 'Swahili that has been mixed with Sheng', which was used by one of the group members (Form Four focus group 3, 
11/2011--Nakuru). Mwelusi responded that Sheng is a mixture of grammars of

different languages, which do not fit well into one. He contrasted Sheng with Swahili, arguing that Swahili has its own stable grammar but Sheng does not. These claims are captured in the following extract:

1. MW: Na tukisema eti kuna Kiswahili kimemixiwa na Sheng, hapo ni kama Sheng na Kiswahili ni lugha tofauti. Ama inamaanisha nini?

2. Mwelusi: Ehe, kwangu ningesema Kiswahili ni lugha inawezajisimamia pekee, lakini Sheng ni mkusanyiko wa lugha ambao tena huo msanyiko hauko kwenye sarufi inayostahili. Unakuta imechangwanywa hata mtu akitaka anatumia kizungu na Kiswahili bado sarufi ya hizo mbili haingiani. Kwa hivyo Sheng ni mkusanyiko wa lugha nyingi na tena zizizofuata kanuni za lugha moja. Lakini Kiswahili ni lugha inayojisimamia yenyewe kisarufi.

(Form Four focus group 3, 11/2011--Nakuru)
1. MW: And when we say Swahili that has been mixed with Sheng, it is like Sheng and Swahili are different languages. Or what does that mean?

2. Mwelusi: Ehe, I would say that Swahili is a language that can stand on its own, but Sheng is a mixture of languages whose grammar is not appropriate. You will find it is so mixed that even if one uses English or Swahili, you will find the two grammars are not matching. Therefore, Sheng is a mixture of many languages that do not follow grammatical rules of one language. But Kiswahili is a language that is self-reliant or can stand on its own from a grammatical point of view.

Next, I focus on the category of speakers who perceive Sheng as a variety of Swahili.

\subsubsection{Sheng as a nonstandard variety of Swahili}

I asked Mrs. Kasisi, a teacher of English in Nakuru, to comment on the language that students spoke most of the time. She responded that they spoke Swahili that "cannot be called standard" (Mrs. Kasisi 10/2011, interview). Later in the interview, she reported that students mainly spoke Sheng, especially in the classroom corridors and when in the playing field. The fact that Mrs. Kasisi's response to my question progressed from Swahili to non-Standard Swahili then to Sheng, suggests that she perceived Sheng as a variety of Swahili. I posed the same question to Mrs. Tajiriba, head of the languages department in the same school, who also defined Sheng as a nonstandard variety of Swahili as shown in the following extract: 
Kiswahili, and the type of Kiswahili that they speak is the Sheng...the Sheng is mostly spoken in the village...in the estate...so they speak that... when they come to school..., they speak English and Kiswahili...the problem we have is that as much as we want them to speak the Standard Swahili, which we teach and the Standard English..., we have a lot of problems.... (Mrs. Tajiriba 11/2011, interview)

Going by the various examples cited above, I find it important to examine why some speakers regarded Sheng as a variety of Swahili while others perceived it as a distinct language variety. According to Lippi Green (2012) and Silverstein (1979), speakers had different language ideology about the nature of language and its appropriateness. In the case of Sheng, the various categories of speakers have different understandings or perceptions of what Sheng is, and associated social functions. On the one hand, most of those who perceived Sheng as a variety of Swahili define it using pejorative terms such as "broken", "adulterated", “improper", "ungrammatical" or "polluted" Swahili. These negative characterizations are effects of the "standard language ideology" (Lippi-Green 2012). According to Lippi-Green (2012) "standard language ideology" is an idealization that seeks to have one "standard", "uniform/homogenous" language, and is maintained by the dominant societal institutions such as the mainstream media and educational institutions. As such, adherents of the "standard language ideology" perceive any linguistic deviation from the standard as "impure", "deficient" and inappropriate, hence one that should be either corrected or abolished.

On the other hand, respondents who regarded Sheng as a distinct language from Swahili fell into three categories: 1) people who described themselves as Sheng speakers; 2) people who found Sheng unintelligible; and 3) those who identified with other competing language varieties such as lugha ya mtaa or Kiswahili cha Mtaani 'street language' or 'street Swahili'. From analytical point of view, the speakers who 
perceived Sheng as a different vriety from Swahili were promoting in-group solidairy while negotiating distinctiveness from other groups in their host society. It is a way of creating "insider-outsider" or the "us" versus "them" distinctions (Gumperz 1982). Those who found Sheng unintelligible, hence different, were doing the same thing but in reverse: they were defining themselves in opposition to people they saw as Sheng speakers, whom they regarded as an out-group. The same was true of those who identified with competing varieties, whether or not they claimed to understand Sheng. So, all these categories of speakers were using Sheng to define boundaries between social groups.

Despite the various categorizations of speakers above, even those who had similar definitions of Sheng did not all agree on what would count as Sheng. In fact, there was more agreement among speakers on what Sheng is NOT rather than what Sheng is. In this regard, Sheng resembles other urban youth vernaculars such as Nouchi in Abidjan, whose definition is equally problematic. Specifically, Newell (2009: 161) argues that, "Nouchi exists in that all Ivoirians believe it exists; but it has no objective, identifiable existence, in the sense that there is no agreement on precisely what Nouchi is, but rather a continual struggle over the right to define it" (see 2.4.0). In the context of my research, I observed that some expressions would be called Sheng in one social group/context but be rejected in another. For example, I presented a pamphlet with two different phrases written in bold letters to student Faridzeh's focus group introduced earlier. I asked one student called Thomas to read the two samples one at a time. The first one was Tia Zi! Drugs Si Poa 'Say no to drugs! They are not good'. I then sought comments from group members about the 
language variety in which those words are written. All the students who responded, i.e., Jane, Joyce and Faridzeh said that it was Sheng because of the word Zi!

However, these students differed in the language variety used in the second sample which is Wakisay Everyone Is Doing It, Washow I Am Not Everyone I Am Myself, or 'If they say everyone is doing it, show them i am not everyone, i am myself'. While Jane and Joyce defined it as Sheng, Faridzeh claimed that it is Heng and gave reasons. ${ }^{26}$ Their differences are captured in the following extract:

1. MW: Eeh. Hebu soma hiyo nayo.

2. Jane: Wakisay everyone is doing it, washow I am not everyone, I am myself.

3. MW: Hiyo ni lugha gani?

4. Joyce and Jane: $N i$ Sheng

5. MW: Ni Sheng?

6. Jane: Eeh

7. Faridzeh: I think ni Heng

8. MW: Hebu patia Faridzeh. Nasikia ako na maoni tofauti. Umesema ni Heng kwa nini?

9. Faridzeh: Mi nimesema ni Heng coz like for example hii "waki" ni Kiswahili. And then "say" ni English. Ok, wakisay everyone is doing it washow I am not everyone. Like "wa" ni Kiswahili bado. I think this is Heng.

10. MW: Hii si Sheng?

11. Faridzeh: Hii si Sheng.
1. MW: Eeh. Read that one.

2. Jane: If they say everyone is doing it, show them that I am not everyone, I am myself.

3. MW: What language is that?

4. Joyce and Jane: It is Sheng

5. MW: Is it Sheng?

6. Jane: Eeh

7. Faridzeh: I think is it Heng

8. MW: Give it to Faridzeh. I hear she has different views. Why did you say it is Heng?

9. Faridzeh: I have said it is Heng because, like for example this "waki" is Kiswahili. And then "say" is English. Ok, "wakisay everyone is doing it, washow I am not everyone. Like "wa" is Kiswahili still. I think this is Heng.

10. MW: So, this is not Sheng?

11. Faridzeh: This is not Sheng.

(Form Two mixed-gender focus group 10/2011--Nakuru)

While the extract above demonstrates that speakers lack consensus on what counts as Sheng, it also alludes to the fact that speakers use different labels to refer to practices that are hard to distinguish linguistically. This is similar to the case of Sheng and Nairobi Swahili discussed earlier. However, owing to the sociolinguistic differences between Nakuru and Nairobi, it is important to examine why speakers in Nakuru employ such labels. For example, I asked student Mpenda, introduced earlier, whether there are other language-use practices included in the mamixture besides

\footnotetext{
${ }^{26}$ The term Heng was not in common use, but I inferred from Faridzeh's explanation that she was referring to a mixture of English and Swahili without linguisistic manipulations other than affixation.
} 
Kiswahili, English and Sheng. She claimed that some students speak "Kiswahilikizungu, which is different from Sheng" (Mpenda 9/2011, casual conversation). According to Mpenda, Kiswahili-kizungu involved drawing words directly from English and attaching Swahili affixes to them as in the word kubeatiwa, which means to be beaten. But how is Kiswahili-kizungu different from Sheng? Table (3) below contrasts some of the Sheng and Kiswahili-kizungu examples that Mpenda gave.

Table 3: A comparison of Kiswahili-kizungu and Sheng

\begin{tabular}{|l|l|}
\hline Kiswahili-kizungu & Sheng \\
\hline Ku- beat- iw- a & Ma-mixture \\
Inf. -beat-Passive-fv & 6- Mixture \\
To be beaten. & Label for different languages used in a single conversation. \\
\hline \multicolumn{2}{|c|}{ (Mpenda 9/2011, casual conversation) } \\
\hline
\end{tabular}

A closer look at the two examples in Table (3) above reveals that it is hard to differentiate between Kiswahili-kizungu and Sheng linguistically. However, the fact that Mpenda, a speaker who identified herself with Sheng, claimed to be able to differentiate the two closely-related linguistic practices suggests that there are social factors involved in the definition of Sheng. Possibly, speakers such as Mpenda belonged to several social groups with different or opposing language ideology. Therefore, her use of different labels to refer to similar linguistic practices may be an attempt to negotiate a balance between the competing desires to belong or associate with the various groups. Hereafter we will use the term "communities of practice" to refer to this type of social group. According to Eckert and McConnell-Ginet (1992:464):

Community of practice is an aggregate of people who come together around mutual engagement in an endeavor. Ways of doing things, ways of talking, beliefs, values, power relations - in short, practices - emerge in the course of this mutual endeavor. As a social construct, a community of practice is different from the traditional community, primarily because it is defined simultaneously by its membership and by the practice in which that membership engages. 


\subsubsection{Sheng as a language variety of particular social groups}

Several informants like Mr. Mpoa and student Kadete from Nakuru described

Sheng as a language of the youth. However, their definitions emphasized or

foregrounded different aspects of youth identity. I discuss these definitions starting

with Mr. Mpoa. I had asked him to comment on the language that students spoke

when they did not have a teacher in class and he responded that they spoke

Swangenglish, which he described as a mixture of English and Swahili. Also, he

referred to Swangenglish as an age-marking language spoken by young people mainly

school-going youths in peer socialization and to express their desire for

“modernity.”In addition, he said that Swangenglish was not standard and gave

specific examples as shown below:

1. MW: When students are not having a teacher in class, is it common to find them speaking Swahili or English? Or what language do they use?

2. Mr. Mpoa: It is common; it is Swahili, English and a bit of their language; the mixture of Swahili and English, Swangenglish. Ile yao wanazungumza katika daraja lao. Wanaitumia sana ile. Utapata mtu anasema "saseni." Sasa hizo ni salamu. Sasa, sijui kama hizo ni salamu rasmi hizo $?^{27}$ Ndio wanatumia sana. Hiyo Swangenglish. [...]Swangenglish, Swangenglish. Inapendwa sana na vijana wa kileo sana hivi.

3. MW: Eeeh

4. Mr. Mpoa: Mmh. Kingereza kidogo, Kiswahili kidogo ili mradi tu tunawasiliana

5. MW: Ooh, very popular among young people?

6. Mr. Mpoa: Mmh, youth. Very popular especially the school-going youths.

1. MW: When students are not having a teacher in class, is it common to find them speaking Swahili or English? Or what language do they use?

2. Mr. Mpoa: It is common; it is Swahili, English and a bit of their language; the mixture of Swahili and English, Swangenglish. Their language; the one they speak at their level. They use that one a lot. You will find a person saying, "saseni" Now those are greetings. Now, I doubt how official those greetings are. That is what they use often. That Swangenglish [...] Swangenglish, Swangenglish. It is really liked by the modern/current generation

3. MW: Eeeh

4. Mr. Mpoa: Mmh. A little English, a little swahili provided that we are communicating.

5. MW: Ooh, very popular among young people?

6. Mr. Mpoa: Mmh, youth. Very popular especially the school-going youths.

(Mr. Mpoa 10/2011, casual conversation)

For student Kadete, I had asked him to describe Sheng and he foregrounded the following aspects of youth identity in his response: 1) Sheng is a language of young people; 2) it is their own innovation through various linguistic manipulations

\footnotetext{
${ }^{27}$ Note that this question is sarcastic.
} 
such as syllabic reversal; 3) it embodies egalitarian language ideology; and 4)

speakers use it to exclude unintended audience. The extract below captures these

sentiments:

1. MW: Nakumbuka tulikuwa tumeongea about "Sheng ni nini?" Lakini leo ningetaka tuone kwa vile Kadete hakuweko, hebu tuambie Sheng ni nini Kadete?

2. Kadete: Sheng naweza sema hii ni lugha tumeunda, vijanaa. Maneno zingine zinageuzwa inakuwa reversed. Inaweza kuwa ni jina kaa gani? Inakaa fatha. Tunasemaga thifa na ni fatha bado. Sasa mimi nikisema thifa mzee labda hatashika nimesema nini. Brother zangu na sister zangu wanajua nimesema nini. Sasa hii ninaweza sema Sheng ni sisi tumeunda. Mtu anakuja tu na majina zake na inakuwa tu ni hivo.
1. MW: I remember we had discussed about what Sheng is but since Kadete was not there, let us hear his views. Kadete, please tell us what you understand by Sheng

2. Kadete: Sheng I can say is a language that we have made ourselves; the youth. Some words are reversed. Let's say like what? It can be like what name? Like "fatha." We usually say "thifa" and it is still "fatha." Now, if I say "thifa," my dad possibly will not understand what I have said, but my brothers and sisters will do. That way, I can say that it is us who have made Sheng. Someone just comes up with his own words and it becomes Sheng

(Form Three focus grou, 10/2011--Nakuru)

The two transcripts above demonstrate that some speakers associate Sheng with particular social groups. Consequently, the attitudes and associations that people have towards these social groups are carried over to Sheng. Such a situation would best be understood by use of Irvine's (2001: 33) notion of iconization which, as earlier noted "is the attribution of cause and necessity to a connection between linguistic behaviors and social categories-of people or activities.” Associations like these contribute to both the rejection of Sheng by school authorities and its embrace by young people as a language of resistance to authority. For example, on the one hand, when young Kenyans claim that Sheng is their language, they not only distinguish themselves from the rest of the society, especially adults, but also claim to be in control of a linguistic resource and everything positive associated with it. As such, the knowledge of Sheng is a form of cultural capital that generates symbolic power among speakers. In this context, speakers of Sheng regard nonspeakers as their 
opposite and label them perjoratively using labels such as washamba, meaning "country."

On the other hand, associating Sheng with certain social groups, especially among non-speakers, is not always positive. For instance, it is common among adults and figures of authority such as teachers, school monitors and parents to disparage Sheng. Recall how some senior prefects in Nakuru and Mombasa associated Sheng with specifici social groups such as Marui and Choka mbaya. Also, some generalized that Sheng is a language of the youth. However, such associations do not acknowledge the following facts: 1) not every speaker of Sheng has discipline problems or performs bad academically; 2) not all young people in Kenya speak Sheng; 3) there are some adults and certain figures of authority such as teachers and parents who speak Sheng; and 4) not all youth languages are called Sheng. Instead, there are other competing variants. In the context of style, Irvine (2001) employs the notion of erasure to describe attending to one dimension of sociolinguistic phenomena (or persons or activities) while rendering others invisible. In this context I ask, what language do young people in Mombasa speak? Do they speak Sheng, a different language variety or both? I engage these questions in the following section.

\subsubsection{Definition of Sheng along geographical lines}

One other aspect along which speakers define Sheng is geographical location, which is closely tied to speakers' negotiation of local identity. For example, while the majority of students in Nakuru claimed to be speakers of Sheng, they told me that people in Mombasa do not speak it. Instead, they claimed that the majority of Mombasa residents, especially adults, speak a coastal variety of Swahili that the 
Nakuru students call kikostoo. ${ }^{28}$ They also claimed that most young Kenyans in Mombasa speak kisheng. As mentioned earlier, some of my friends in Nakuru and Mombasa had tried steering me away from Mombasa and suggested that I consider doing my research in Nairobi, because that is where I would find Sheng. This association of Sheng with the urban areas in the upcountry, like Nakuru and Nairobi and KiSheng with Mombasa was also echoed by Mr. Mpoa. He is originally from the coast but resides in Nakuru. According to Mr. Mpoa, Sheng is different from $K i$ Sheng, in that, while KiSheng is a "mixture of perfect, unadulterated Standard Swahili and English, Sheng is Swahili but with vocabulary coined from other sources only known by speakers" ${ }^{29}$ (Mr. Mpoa 10/2011, casual conversation). Mr. Mpoa added that while Sheng was spoken in Nakuru, KiSheng was spoken in urban areas along the Kenyan coast and Tanzania.

Despite associating Sheng with Nakuru, Mr. Mpoa told me that speakers of Sheng in Nakuru mostly copy the behaviors of Nairobi youth, especially from media entertainment programs such as Beba beba, which is produced in Nairobi but airs on one of the National TV stations. While it is true that media contribute to the diffusion of Sheng to social groups outside Nairobi such us through Hip Hop music (Samper 2002), some speakers of Sheng in Nakuru would dispute Mr. Mpoa's claim that they usually copy Nairobi youth. For example, recall that students in Bakari's focus group in Nakuru claimed that the word kadete 'talking too much' was invented by one of their friends. Later on, they told me that they called him Kadete in order to

\footnotetext{
${ }^{28}$ The prefix $k i$ - in this case indicates that Kikostoo is a language. Use of the prefix $k i$ - is the Swahili convention for marking languages or manners of speaking associated with social groups (Hinnebusch and Mirza 1998). As such, Kikostoo in my research is a language variety that speakers of Sheng associated with people from coast. The English word "coast" has undergone some phonological alterations before being used in Sheng, hence Kostoo. Prefix $k i$ - is also used to mark diminutives in Swahili (Hinnebusch and Mirza 1998).

${ }^{29} \mathrm{Mr}$. Mpoa reported that KiSheng is also called Swang or Swanglish in Mombasa and Tanzania.
} 
acknowledge his innovativeness. Similarly, a male student in a mixed-gender focus group claimed that the word tintole 'darkness, e.g., at night') is only used in his residential neighborhood. Therefore, Mr. Mpoa's claim would not hold among such speakers of Sheng in Nakuru. However, regardless of whether Sheng in Nakuru comes from Nairobi, there was a general agreement between informants in Nakuru and Mombasa that the language variety spoken by many young Kenyans in Nairobi is Sheng, while many young Kenyans in Mombasa speak either KiSheng or lugha ya mtaa.

In Mombasa, there were ethnic differences in the way speakers employed these labels: while speakers from the communities that regard themselves as coastal called it lugha ya mtaa, those from the communities perceived as belonging to the upcountry called it KiSheng. But of what relevance is this Nairobi-Mombasa dichotomy in the context of Sheng? How is Sheng different from the lugha ya mtaa associated with Mombasa? The Nairobi-Mombasa dichotomy is important in the context of defining Sheng because it points to the underlying tensions and stereotypes between coastal and upcountry people in Kenya. One student called Maunga from student Zawadi's focus group identified himself as a speaker of lugha ya mtaa. He reported that he initially thought that Sheng and lugha ya mtaa were the same. He, however, learned that the two language varieties were different when he visited his relatives who resided in Nairobi (Form Two focus group 2, 03/2012--Mombasa). He told me that he went to a road-side kiosk but experienced miscommunication with the vendors when he ordered French fries worth dala 'five shillings'. He said that the vendors put on faces of confusion that suggested they were not only wondering what he meant, but also where he came from (origin). Interestingly, when Maunga 
explained that dala meant five shillings, one of the vendors then asked him, "Ooh! You mean five bob?" Maunga responded in the affirmative and another question followed, “Umetoka coast wewe?”, 'Do you come from the coast?’ Maunga responded in the affirmative and the vendor said, "Sasa tumekuelewa", "we now understand you'.

The vendor's statement "sasa tumekuelewa" immediately after Maunga confirmed he is from the coast is multivocal: at one level, the vendor learned that dala meant five shillings. At another level, the vendor acknowledges that Maunga spoke a different language variety. Lastly, and this is the most relevant interpretation in the context of Sheng, the vendor must have considered both the vocabulary and Maunga's pronunciation (accent) to stereotype or put him in a particular social category, i.e., "somebody from the coast." So, how do these linguistic differences between Sheng and lugha ya mtaa and the geographical associations that speakers make to the two language varieties play into the underlying tensions and stereotypes between coastal and upcountry people?

In Kenya as in many parts of the world, youth as a social category is perceived as if it is a confined, homogenous segment of the social fabric defined by biological age. However, youth in actual practice is a highly fluid and heterogeneous social category, and biological age only applies in very limited contexts. In the case of Sheng, most Kenyans, especially non-speakers, define it as a language of the youth. Such a definition as noted earlier erases (in the sense of Irvine 2001) several factors that are relevant to the definition of youth. For example, when young Kenyans in Mombasa claimed to speak lugha ya mtaa while associating Sheng with Nairobi, they were claiming to be a distinct social category from the youth in Nairobi. I inferred 
from our conversations with student informants in Mombasa that language was not the only distinguishing factor. For example, student Zawadi, mentioned earlier, outlined some of these differences as follows: coastal youth like being free and independent; they generally dislike being accompanied by their parents especially mothers, and do not rely on their parents to wash their clothes. Also, coastal youth are "tough" in that, "Kwao mchana huanza usiku”, 'for them, the day begins when night falls'. They usually go to the beaches late in the evening when everybody else including adults, is leaving for home. In addition, Zawadi reported that many young people at the Coast used hard drugs. On the contrary, Zawadi equated youth in Nairobi with women's purses, because they were usually accompanied by their mothers and relied on them to do their laundry. As such, Zawadi said that Mombasa youth generally refer to Nairobi youth as "Mama's boys" (Form Two focus group 2, 03/2012--Mombasa).

The various contrasts that Zawadi draws between coastal and Nairobi youth points to the fact that speakers of Sheng and of competing variants define themselves in opposition to each other using "acts of alterity" (Newell 2009). This is similar to what happens among speakers of other urban youth vernaculars in Africa such as Nouchi. For example Newell (2009: 164) observes that:

Nouchis are defined not only by reference to stereotypical positive models, but also in contradistinction to stereotypical others, such as the loubards, a divergent form of youth culture related to but not identical to the nouchi...Although nouchi and loubards interacted continually, often as part of the same groups and networks, they distinguished themselves from one another.

In sum, this section has focused on the problematic associated with the definition of Sheng. It has clearly pointed out that what speakers call Sheng cannot be adequately defined or distinguished from other closely-related varieties such as 
Nairobi Swahili, Heng, KiSheng or lugha ya mtaa solely on a linguistic basis.

Therefore, I find previous scholarly attempts to define Sheng inadequate because they sought to fit it into the available linguistic categories such as dialect, code-switching and pidgin rather than employ multiple approaches or seek to know how speakers themselves define Sheng. Instead, I have argued that Sheng is socially defined, hence the need to consider social factors such as speakers' attitudes, their identities and language ideology.

In order to account for both linguistic and social factors that speakers employ when defining Sheng, I have argued that the notion of style specifically as a "social semiosis of distinctiveness" (Irvine 2001: 23), is the most appropriate theoretical approach. By use of style, I have demonstrated that what speakers call Sheng is contextual. Consequently, an individual speaker may give different and sometimes conflicting definitions of Sheng. I have attributed these contradictions to speakers' language ideology, which is mostly influenced by an individual speaker's membership in multiple communities of practice (Eckert and McConnell-Ginet 1992). Such co-membership suggests that individual speakers may have multiple and sometimes competing social identities which affect their stance towards Sheng at any given moment.

Even though this chapter primarily focuses on the definition of Sheng, the Sheng phenomenon and the contradictions inherent in its definition are not peculiar to Kenya. Instead, there are other African urban youth vernaculars such as Nouchi in Abidjan (Newell 2009) that resemble Sheng in terms of structure, social functions, and pervasiveness in peoples' conversations, language ideology and contradictory 
norms. These similarities are well captured in the following extract from a conversation between speakers of Nouchi, in Abidjan:

Henri: People here grow up hearing so much Nouchi, they can never even arrive at mastering French. Only those who get to Troisieme [University Level] even get close. We just can't help mixing Nouchi in.

Olivier: Don't other countries have Nouchi too?

Henri: All countries have some kind of Nouchi, but not as strong as ours. Ours is a real language. It is part of what makes Ivorians different from other Africans. (Newell 2009: 178)

I outline a few of these African urban youth vernaculars and how they relate to the Sheng phenomenon in Kenya in the following section.

\subsubsection{Sheng and other urban youth vernaculars in post-colonial Africa}

Contemporary urban Africa is ethnically and linguistically heterogeneous (Kiessling and Mous 2004; Makoni et al., 2007; McLaughlin 2009; Newell 2009). However, these scholars observe that most young people find the available language varieties unsuitable for their communication purposes, and for expressing desired distinct social identities. For example, ethnic languages may be loaded with connotations of ethnic antagonism, which might be offensive to some members of their social networks. Also, the youth associate ethnic languages with older generations and traditional way of life from which they seek to distance themselves. Also, young Kenyans find the former colonial language varieties such as English in Kenya and French in Abidjan unsuitable, because they are dominated by the elite whose values and practices stand in opposition to the youth. Therefore, youths in urban Africa have been innovating their own language varieties from the existing linguistic resources such as Sheng in Kenya, Nouchi in Abidjan etc., as a response to 
globalization and the challenges of post-colonial city life (McLaughlin 2009; Samper 2002).

Some of these challenges include youths' desire to have a common medium of communication among speakers from diverse ethnic backgrounds, and independence from parents and other figures of authority. These challenges are perpetuated by youths' liminal status since they are neither children nor adults from a societal point of view. Due to youths' quest for independence, the society constructs them socially as a threat to the established social order. However, there are contexts where the youths' desire for recognition makes them a formidable force in nation building, and driving social change such as during political elections. Ironically, political aspirants see them in terms of numbers or votes. Other interested parties like the advertisement and entertainment industries perceive youth as a potential market for their finished products. Consequently, it has become very common for such interested parties to strategically use the language variety associated with the youth to woo them, a situation that renders the youth even more liminal. This is well articulated by Argenti (2007: 9) who says that:

\footnotetext{
What youth have in common is not their age but their exclusion from power and their dependence on the 'men', 'fathers', or 'elders' in their societies. The category of youth was and is .... movable feast, a derogatory term masquerading as flattery and used by those in positions of power to define ever-shifting groups of subordinate people.
}

Different scholars observe that African urban youth vernaculars have a lot in common in form and function (Kiessling and Mous 2004; Makoni et al., 2007; Spitulnik 1999). Speakers employ various innovative linguistic strategies at the morpho-phonological, semantic and syntactic levels to generate new forms from their 
linguistic repertoire. At the morpho-phonological level, these language varieties like Sheng, usually employ processes such as affixation, reversal of syllables, borrowing, truncation and coining new vocabulary. At the semantic level, common strategies include semantic reversal which is assigning the borrowed word a meaning that is opposite to its original. There is also semantic expansion, which refers to expanding the meaning of a borrowed word to include nuances that were not included in its source language. At the syntactic level, the youth employ ingenious code-switching and mixing. The innovated linguistic material is later embedded in one of the dominant languages, which serves as the matrix language (Myers-Scotton 1993). ${ }^{30}$ The various linguistic manipulations make these youth vernaculars appropriate for serving different social functions such as the ones discussed below.

\subsubsection{Main social uses of urban youth vernaculars}

\subsubsection{Negotiating speakers' desired social identities}

Speakers of these language varieties employ them primarily to distinguish themselves from the rest of the society and simultaneously promote in-group solidarity. For example, Spitulnik (1999:32) argues that Town Bemba has multiple variants and each of them indexes a different social identity. That is:

There are in a sense several registers of Town Bemba: a Street Town Bemba, an Elite Town Bemba, a Smooth Town Bemba, and a Common or Everyday Town Bemba. All of these varieties have strong connections with notions of 'modem urban life' in contrast to 'traditional rural life', but they also can have widely differing social connotations: a rough, economically harsh, and even criminal subculture; a trendy and playful youth subculture; a sophisticated, cosmopolitan lifestyle; or simply, the generic urban orientation.

\footnotetext{
${ }^{30}$ Myers-Scotton (1993) defines matrix language as the language that provides the grammatical framework within which innovated linguistic material is embedded.
} 
Besides linguistic materials, urban youth in Africa appropriate other social metasigns to negotiate distinctiveness. These metasigns include music, dance, clothing, hairstyle, comic books and other popular literature, ways of walking and preoccupation with international culture, especially African American "pop" culture (Kiessling and Mous 2004:316-317; Spitulnik 1999:47). However, since these speakers are not socioeconomically and politically powerful, they usually modify the locally available materials to make them desirable, or buy second hand clothes that have been imported from foreign countries. They then give these foreign materials new local meanings. As a result, various scholars such as Newell (2009) and Samper (2002) argue that identities negotiated using urban youth vernaculars such as Nouchi and Sheng are simultaneously global and local.

According to Kiessling and Mous (2004), speakers' desired distinctiveness involves reversal of norms and develops from a resistance identity, which is associated with subordinated social groups to a "project identity," which is aimed at reforming the society. Consequently, these urban youth vernaculars are in an antagonistic relationship with the state-sanctioned languages. For example, Newell (2009: 158) reports that:

\footnotetext{
Nouchi is thus a case of a language moving against the current of social hierarchy, travelling from the 'corrupted' French of Ivoirian society's most marginal members to a form of discourse associated with Ivoirian national pride, a journey filled with contradiction, ambivalence, and conflicting evaluations. The Ivorian state has encouraged a worldview of modernity centered on French language and culture, and the evaluation of speakers in terms of the purity of their French has been an instrument of class reproduction. Nouchi provides an alternative to this state-imposed modernity of externality, locating modernity within urban Ivorian speech.
}

From a linguistic point of view, speakers of these urban youth vernaculars such as

Nouchi reverse the norms by mixing and switching between the various language varieties in their repertoire, and innovatively manipulating the form and meaning of 
borrowed lexicon (Abdul-Aziz and Osinde 1997; Kiessling and Mous 2004; Makoni et al. 2007; Newell 2009; Spitulnik 1999). The various scholars argue that these linguistic practices enable speakers to simultaneously transcend ethnicity, mark different social identities such as urbanity and modernity, and make their conversations unintelligible to non-speakers. In Zambia for example, Spitulnik (1999: 47) says that, "the innovations and experimentations within Town Bemba are both iconic and indexical of 'newness' and a flaunting of 'tradition.' This newness and deviation have symbolic value in contemporary Zambia, as part of the meaning of modernity." I now turn to the claim that use of urban youth vernaculars enable speakers transcend ethnicity.

\subsubsection{Bridging interethnic differences among speakers}

As mentioned earlier, contemporary urban Africa is ethnolinguistically heterogeneous. The various urban youth vernaculars bridge ethnic differences among their speakers, but there is not a consensus among scholars about how they do so. Some scholars such as Githiora (2002) and Makoni et al. (2007) argue that these language varieties bridge ethnic differences by incorporating words from the various ethnic languages spoken in the city. Other scholars such as Kiessling and Mous (2004: 315-316) argue that "it is the modernity and the urban status of youth languages that dissociate them from ethnic associations." Both of these factors may be playing a role.

\subsubsection{Challenging established social norms}

Different scholars have used different terms to refer to these urban youth vernaculars due to their antagonistic relationship with the dominant languages and 
established authority. For example, Kiessling and Mous (2004) classify them as "antilanguages," a term that was coined by Halliday (1978) to refer to language varieties that arise and function in opposition to the established linguistic norms, thus creating a counter reality. According to Halliday, speakers of an antilanguage constitute an antisociety because its values are usually in opposition to those of the mainstream society. Kiessling and Mous (2004) refer to African urban youth languages as antilanguages for various reasons related to their form and functions. From a functional point of view, the two scholars claim that the majority of these language varieties such as Nouchi, Sheng, Indoubil, Iscamto and Tsotsitaal emerged among street gangs or among operators of the underworld who needed a coded language for their secretive deals. A good example that reveals the close connection between these language varieties and the occupation of their speakers is Tsotsitaal: it is coined from tsotsi, 'criminal' and taal 'language', hence Tsositaal 'criminal language' (Kiessling and Mous 2004: 310).

Two other attributes that characterize African urban youth vernaculars as antilanguages are: the social position of their speakers in relation to the mainstream society; and 2) the parasitic relationship between each of these language varieties and the dominant language within which it is embedded. As a result, Kiessling and Mous (2004: 314) argue that speakers simultaneously associate and dissociate themselves with the dominant group, hence operating in a "paradox of norms."

Speakers also employ these language varieties to challenge political authority as is the case with Town Bemba (Spultinik 1999). According to Spultinik (1999:40), "from its beginnings, the linguistic makeup and sociolinguistic valency of Town Bemba has 
reflected and responded to the complex politics of African urbanization, identity formation, and colonial contact." Similarly, Richardson (1964:190, cited in Spitulnik 1999:40) describes Town Bemba as a political symbol that speakers use to consciously react to traditional authority by abandoning traditional modes of speech and adopting a new intertribal language, which unites town Africans against all newcomers. But what happens when speakers of these urban youth vernaculars grow up?

There are different possibilities given the heterogeneous nature of speakers and the multiplicity of functions that these urban vernaculars play. That is, some speakers either abandon or adopt them for wider communication since the upcoming generation will innovate their own variety to set them apart from the older generation (Kiessling and Mous 2004; McLaughlin 2009). However, there are some speakers such as Manambas and music artists in the case of Sheng, who continue using these language varieties because they are the main media of communication in their occupations. Therefore, different sections of the host society evaluate these language varieties differently. I discuss these attitudes below.

\subsubsection{Attitudes expressed toward African urban youth vernaculars}

There is a similarity in the kind of attitudes expressed toward Sheng and other urban youth vernaculars in different parts of urban Africa. These attitudes range from negative, positive to ambivalent. Speakers of these language varieties evaluate them positively because they are both sources of covert prestige ${ }^{31}$ (Labov 1972). Also,

\footnotetext{
${ }^{31}$ Covert prestige is associated with an in-group that does not enjoy prestige in the wider society and its use conveys symbolic meanings such as rebelliousness, which is valued by that in-group.
} 
speakers find them suitable for serving different social functions such as those outlined in subsection 2.4.1. Different scholars such as Newell (2009) have reported that some speakers are optimistic about these language varieties becoming their countries' future national languages, e.g., Nouchi in Abidjan. There are also nonspeakers, especially linguists, who evaluate these youth vernaculars positively because they understand their heteroglossic nature (Githinji 2006; Samper 2002). ${ }^{32}$

Some sections of the society evaluate these language varieties negatively. Such evaluations are usually rooted in the hidden power struggle between speakers of these language varieties who usually occupy subordinate positions in their societies and the adherents of of the dominant "standard language ideology" (Lippi-Green 2012). As mentioned earlier, "standard language ideology" is usually promoted by dominant institutions such as schools and figures of authority such as parents and government officials. Consequently, the effects of "standard language ideology" have led some people to associate the standard language with good quality, "correctness" and communicative adequacy while defining the nonstandard ones as the opposite. For example, some teachers and parents in contemporary urban Africa describe nonstandard language varieties spoken by the youth using demeaning terms such as slang, "corrupted" and "lazy" way of speaking (Kiessling and Mous 2004; McLaughlin 2009). Such characterizations not only depict these language varieties as "impure" and inappropriate, but also deny them the status of a language, which is associated with socio-political power (Lippi-Green 2012). A good example is Town

\footnotetext{
${ }^{32}$ Bakhtin (1982) defines heteroglossia as the multilayered nature of language in which there are opposing centripetal and centrifugal forces. In actual practice, centripetal forces refer to the efforts put by governments and education systems to promote the use of a unified, standardized language while centrifugal forces refer to the pressures of social differentiation within a language, which are manifested in its speakers' desire to negotiate different forms of social identity such as class, gender and urban sophistication.
} 
Bemba in Zambia, which has been historically mischaracterized as a reduced or simplified language despite its linguistic complexity (Spitulnik 1999). According to Spitulnik (1999: 34), such mischaracterization could be informed by "the stereotypes and misconceptions that inform Western thinking about both multilingualism in general and colonial and post-colonial speech communities in particular.”

The last category of speakers assumes contextual stances toward these urban youth vernaculars. While the ambivalence in speakers' attitudes may be attributed to co-membership in multiple communities of practice, non-speakers' ambivalence as is the case with teachers in charge of guidance and counseling in Kenya, may be attributed to how Sheng enables them to reduce social distance when interacting with student clients. From a different point of view, I argue that non-speakers' ambivalence may be an indicator that society's attitudes toward these language varieties are changing with time. For example, in the case of Nouchi, Newell (2009: 158) reports that, "today Nouchi is not only spoken with pride and imitated widely by youth of all classes and from all over the country as well as the Ivorian media aimed at them, but more and more often it is the language spoken at home and thus the first language of many Ivorians.” Sheng in Kenya has taken a similar trend; some national media stations such as Citizen TV, have introduced programs produced in Sheng such as Beba beba and Kubamba mainly targeting young people. The new Swahili curriculum has also introduced a section called Isimu Jamii, Swahili for 'sociolinguistics', aiming to enhance linguistic awareness about how people make language choices in their day-to-day interactions among other issues. I asked some teachers to provide me with samples of national exams, and I saw questions asking students about Sheng and how 
it relates to other languages such as Standard Swahili. These developments together with the increased strategic use of Sheng among politicians and Christian religious organizations may indicate that the society in general has expanded social space for the youths and their language varieties as well (see Chapter Four).

In sum, this chapter has examined the contested definition of Sheng among its speakers. I have demonstrated that Sheng is a moving target given that it was originally produced and continues to be reproduced by speakers seeking to exclude unintended audiences from in-group communication and to promote in-group solidarity. In this context, each category of speakers defines Sheng in a way that distinguishes it from other competing varieties, hence create and maintain the "us" vs. "them" boundaries. Consequently, each of these categories claim to be speakers of the "real" Sheng while demeaning other varieties, using labels such as KiSheng, Heng, lugha ya mtaa and Swangenglish. However, the idea of solidarity vs. exclusion in Sheng does not assume fixed social groups. Instead, Sheng is a kind of border-work, which positions speakers in groups that shift to enhance its impermeability to unintended observers. Therefore, speakers' stances toward Sheng are contextual. Some people would claim to be speakers of Sheng and evaluate it positively in one context, but deny and evaluate it negatively in another. The same applies to speakers who would identify with competing language varieties. This was the case in Mombasa where some speakers who had earlier claimed that they spoke Sheng later identified themselves with lugha ya mtaa. In this context, they associated Sheng with Nairobi youth whom they regarded as "mama's boys", to construct them socially as "soft" and “dependent." This distinction was motivated by speakers of lugha ya mtaa's desire to 
negotiate local identity, especially because there are tensions and stereotypes between coastal and upcountry Kenyans. I have attributed these contextual stances toward Sheng to the fact that social factors such as speakers' desired identities and language ideology influence how they perceive and employ Sheng in their daily interactions. Therefore, I have argued that Irvine's notion of style is a more appropriate analytical tool than the existing linguistic criteria, since it is broad and flexible enough to accommodate the wide range of speakers' understandings of Sheng.

From another point of view, I have demonstrated that the Sheng phenomenon is not peculiar to Kenya. Instead, there are similar language varieties such as Nouchi in Abidjan and Town Bemba in Zambia which serve multiple social functions among speakers. Some of these functions contravene the state-sanctioned national language policy and associated identities. In this regard, authorities in dominant institutions such as schools and government do not approve of the use of these urban youth vernaculars. For example, they claim that use of these nonstandard language varieties interferes with students' mastery of the officially sanctioned languages, further affecting their overall academic performance in national exams. Authorities also associate these linguistic forms with street gangs and other categories of law breakers. Such negative attitudes from the agents of the state is a clear sign that the state feels threatened when speakers embrace these unauthorized linguistic forms such as Sheng in Kenya and Nouchi in Abidjan, while turning away from the officially-sanctioned languages.

Despite the contextual nature of speakers' attitudes toward Sheng, there are some who think that it has a bright future in Kenya. Also, the spread of Sheng to domains 
which were previously associated with Standard Swahili and English such as the mainstream media suggests that the Kenyan society in general is gradually accepting Sheng. These developments call to attention the claim at the beginning of this chapter that Sheng has existed since the early 1960s. This was a time when the postindependence government was seeking to break away from its former colonial master by adopting its own educational structures and national language policy. However, the continued flourishing of Sheng indicates that there is a disconnect between the government's efforts in schools to promote the use of English and Standard Swahili and peoples' language use in actual practice. In fact, there are some scholars of Sheng such as Mugane (2005), who attribute the emergence of Sheng to the inconsistencies in the national language policy and linguistic mismatches between theory and practice. I engage these questions in the next chapter, which traces the development of formal education and the national language policy in Kenya from the colonial period to the present. 


\title{
Chapter Three
}

\subsection{The National Language Policy in Kenya: Theory vs. Practice}

\begin{abstract}
Why does Kenya handle the issues of language the way it does?...Our behavior is politically motivated. It seems that we only appeal to a language of wider communication when we feel threatened. During the colonial period, the same was true. English was only taught to Africans when it became obvious that Europeans could no longer be secure without African 'partners'. Before this realization, Africans were to be denied English language due to its political utility... Looking at the history of official language use, it is clear that we only emphasize the need and the use of the national language when the political system is threatened... as soon as the dust settles, our leaders always forget the language issue. (Mutahi 1979: 9-10)
\end{abstract}

The present chapter focuses on the disconnect between the national language policy and peoples' language use in actual practice. It traces the introduction and development of Kenya's formal education and associated language policy from the colonial period to the present. It is important to focus my analysis from the colonial period because both formal education and the national language policy are parts of the colonial legacy that Kenya inherited from the British at independence in 1963.

\subsubsection{Kenya's formal education and associated language policy during the colonial period (1900-1962)}

Formal education was introduced in the $19^{\text {th }}$ century by European missionaries who had come to Africa to spread Christianity and western values (Eshiwani 1993; Mutahi 1979; Sifuna 1990). They were later joined and supported by British colonial authorities who had come to Africa for econo-political interests. I use the term formal education as used by Eshiwani (1993: 15) to mean, "[w]estern education, where the learning and teaching activities are formalized into a classroom situation.” This definition is significant because Kenyans had their own forms of traditional education and sociopolitical structures before the arrival of the Europeans, but these practices 
and organizational structures varied from one community to the other (Eshiwani 1993; Marah 2006; Ociti 1973). From a general point of view, the various scholars report that African traditional education was organized around social institutions such as the family, where the elder members informally educated the younger ones about the social norms and cultural practices that would make them both acceptable and active participants in the wider society. Traditional education on the heavily Muslim Kenyan coast, however, was centered on the teachings of the Koran, which was conducted in Arabic and in centers of learning called madrassas located within and around mosques (Insoll 2003; Sifuna 1990).

From a sociolinguistic point of view, early missionaries found the complex ethno-linguistic situation in Africa unfavorable for their religious and educational interests. This complexity necessitated creation of convenient categories of identity to facilitate the codification of African languages to be used in translating the Bible and spreading Christianity among Africans. Various scholars argue that the early missionaries had their own ideas against which they framed their understanding of the linguistic varieties they encountered in Africa. They then mapped these ideas onto the Africans and ended up lumping together people with perceived linguistic similarities, creating a very artificial category of identity that they called "tribe" (Gal and Irvine 1995; Hymes 1968; Makalela 2005; Nadel 1947). In this context, each "tribe" was associated with one "ethnic language." A good example of this artificiality is provided by Nadel (1947:13) saying that:

We shall meet with groups which, though they are close neighbors and posses an almost identical language and culture, do not regard themselves as one tribe...; and we shall also meet with tribes which claim this unity regardless of internal cultural differentiation. Cultural and linguistic uniformity, then, does not imply, and cultural 
and linguistic diversity-- at least within certain limits- not preclude, the recognition of tribal unity.

I argue that the notions of "tribe" and "ethnic language" are problematic constructs whose relevance has been contested by various scholars, since they introduced rigid and artificial social and physical boundaries between African communities, which had earlier lived and interacted in fluid ways (Hymes 1968; Gal and Irvine 1995). According to Gal and Irvine (1995: 969), the correlation between "one language" and one "ethnic group" is grounded on the misinformed assumption that "linguistic differentiation-- formation of languages and dialects--is a consequence of the physical isolation of populations that supposedly produces self-evident linguistic boundaries." The two scholars criticize this correlation, arguing that it "erases" other relevant attributes which define social groups besides language. By “erasure," Gal and Irvine (1995: 974) refer to a process through which:

A social group, or a language, may be imagined as homogenous, its internal variation disregarded. Because a linguistic ideology is a totalizing vision, elements that do not fit its interpretive structure - that cannot be seen to fit - must either be ignored or be transformed.

Closely-related to the above problematic, the early missionaries in Africa also employed criterion of mutual intelligibility in which language varieties with perceived linguistic closeness were regarded as "dialects" of the same "language" (Hymes 1968). According to Hymes (1968: 9), the criterion of mutual intelligibility is informed by the false assumption that, "when the units are demarcated in space (mapped), the demarcation implies a break in mutual intelligibility in virtue of the language, and hence in communication in virtue of the culture." However, Hymes argues that this criterion is 
problematic because there are cases where there may be mutual intelligibility despite difference of language and mutual non-intelligibility despite oneness of language. For example, in the Niger Delta, Nigeria, there are several languages which are reported to have diverged from a common ancestor in a contiguous territory. However, when these languages are categorized on the basis of mutual intelligibility, moving from west to east, some are intelligible to their neighbors but not to speakers of varieties that are farther away.

In the Kenyan context, Ogechi (2002) and Githinji (2006) observe that the criterion of mutual intelligibility is inadequate because it admits degrees of intelligibility which can vary greatly, especially because the distinction between a "language" and a "dialect" is political. Consequently, some languages may be called "dialects" in one context but be labeled "languages" in another, and vice versa. For example, Githinji (2006: 16) reports that:

The Bukusu, Nyole and Idakho are regarded as Luhya dialects, yet in Ethnologue map, they are coded as separate languages. Similarly, Tharaka and Mwimbi are normally classified under Meru, while in the map they are usually represented as separate languages. The Sabaot, Pokot and Tugen, normally classified under Kalenjin, are coded as autonomous.

Due to the political nature of the distinction between "language" and "dialect," it is not clear how many ethnic languages are spoken in Kenya. As a result, it is common to find scholars or Kenyans in general reporting different figures; some say there are 42 ethnic languages (Samper 2002), others 70 (Think Africa Press, June $12^{\text {th }} 2012$ ) and so on.

My study also found that there are cases where speakers employ these ethnic labels in ways that do not conform to the correlation between "one language" and "ethnic group." For example, a teacher in Mombasa, Mr. Fujo, 
told me that coastal people use the term "Kikuyu" in protest, when referring to the upcountry communities who own land and other economic resources at the coast (Mr. Fujo 01/2012, interview). According to him, there was a time the first president of Kenya visited the coast after independence, and found coastal communities living in deplorable conditions while sitting on huge tracts of idle land. When the president sought to know why they were not utilizing their land, they told him that they needed tractors to till it. Mr. Fujo told me that the president may have taken their response as an insult, prompting him to sarcastically promise that he would bring tractors. However, he said that the President instead gathered members from his Kikuyu community and allocated them that land. This incident, according to Mr. Fujo, created tension between coastal and upcountry communities, which has continued to the present. It is in this context of protesting the presence of outsiders who own resources at the coast that coastal people employ the label "Kikuyu" to mean both "outsiders" and "exploiters" (Mr. Fujo 01/2012, interview).

In summary, the foregoing discussion has demonstrated that the notions of "ethnic language" and "tribe" used by the early missionaries to codify African languages both in Kenya and beyond are problematic. However, the missionaries found them convenient for pursuing their own religious interests among Africans. Similarly, racial categories such as “Asians", "Europeans", “Arabs", "Africans" and "Waswahili" used during the colonial period were problematic constructs, because the people to whom these labels apply do not perceive or define themselves that way (Eastman 1971; Wald 1985). Also, these labels were categories of exclusion in line with 
the British divide-and-rule policy (Mutahi 1979). In this context, though the main categories of white people in Kenya were the British colonial administrators, settlers and church missionaries, the term "Europeans" was extended to all people regarded as racially white. Also, despite the fact that the label "Asians" could in principle refer to all people from Asia, it was used exclusively to refer to the immigrants from India and Pakistan. Most of these “Asians" had come to Kenya to do business, or were brought by the British colonial administrators as laborers to facilitate the construction of the KenyaUganda railway. The term "Arabs" was used when referring to both business merchants from the Middle East and also to the Waswahili, the inhabitants of the coast who had been there for more than a millennium. The inclusion of Waswahili under the label "Arabs" contributes to their contested ethnicity (see Chapter Four).

The various colonial categories of identity are relevant in understanding the history and nature of Kenya's formal education and associated language policy. Prior to the arrival of the European missionaries, Africans regarded their traditional education highly. However, when the European missionaries came, they socially constructed African education as "heathen" and "retrogressive," because they saw it as an impediment to their efforts to spread Christianity (Eshiwani 1993). Consequently, the Europeans started pressuring Africans to abandon their traditional education and embrace the "better" and "progressive" Western education. However, since the missionaries and colonial authorities had different educational goals and interests, they could not agree on the appropriate medium of instruction, owing to the complex ethnolinguistic situation in Kenya. Language policy therefore became a 
constant site of struggle between the colonial authorities and the missionaries, a situation that led to the formulation of many inconsistent and sometimes contradictory policies throughout the colonial period.

According to Zuengler (1985: 241), such struggles related to language planning are characteristic of multilingual societies because, "[d]ecisions to be made concerning language policy, and in particular the choice of educational language, are neither simple nor universally acceptable." The issue of acceptance is significant because language and education are also intertwined with other aspects of peoples' lives such as politics, identity, economy and traditions. Therefore, language planning in any given society usually reflects competing socio-political values and interests between social groups, and such competition often leads to disconnects between theory and practice. In the next section, I focus on the nature of education for Africans proposed by the European Missionaries on the one hand, and by the colonial authorities on the other. I examine how the competing interests of each of the two categories of Europeans influenced their choice of a language policy, starting with missionary education.

\subsubsection{Missionary education in Kenya.}

The early missionaries in Kenya arrived in three major waves, and all of them aimed at educating Africans primarily in their native (in the sense of first acquired language) languages to help spread Christianity and western values to fellow Africans (Sifuna 1990). In order to achieve these goals, the missionaries had to train Africans how to read and write so that they could communicate easily with missionaries and colonial administrators, and to serve as interpreters (Eshiwani 1993). According to 
Sifuna (1990), the first group was Roman Catholics from Portugal. They came during the Portuguese rule along the coast of East Africa in the $16^{\text {th }}$ and $17^{\text {th }}$ centuries. They first established a monastery in Mombasa in 1557 then worked northwards towards Lamu within the areas controlled by the Portuguese. However, this group of missionaries stayed in Kenya for a very short time, hence they only managed to convert a handful of local people.

The second group of missionaries was Lutherans led by Dr. Krapf who had worked as a missionary among the Galla community in Ethiopia under the auspices of the Church Missionary Society (CMS). His missionary work in Ethiopia was unsuccessful and therefore sought permission from the CMS to follow the same community from an Eastern base at Mombasa (Sifuna 1990). Dr. Krapf was later joined by Johann Rebmann in 1848 and Jacob Erhardt 1849, but they relocated to the friendlier town of Rabai because Mombasa was dominated by Muslims, whom they perceived to be their religious antagonists. According to Sifuna (1990), Dr. Krapf's group established a mission station and a school at Rabai where they embarked on translating parts of the Bible into Kiswahili and Kirabai, and provided the translated reading materials to their pupils to help them prepare for baptism. However, Sifuna reports that the educational progress of Dr. Krapf's group was slow, and their efforts to persuade parents to send their children to school were generally unsuccessful.

The third wave of missionaries was greatly influenced by the early European explorers who actively participated in the anti-slavery movement of the $19^{\text {th }}$ century. For example, Dr. Livingstone's writings, specifically his attacks against the Arab slave trade, initiated conversations in most missionaries' circles about how African slaves were being mishandled by Arab slave traders (Sifuna 1990). Also, Sifuna 
reports that these writings influenced the British government to intensify its anti-slave activities by sending warships to fight slave traders and pressure them to free slaves. The anti-slavery activists of the $19^{\text {th }}$ century sought to abolish slave trade and settle the freed slaves for two main goals specifically geared to serve their own socioeconomic and political interests.

The British government on the one hand sought to establish "legitimate" commerce such as agriculture, to generate raw materials for their industries in Europe and also to start new trading posts for their finished goods. The missionaries on the other hand sought to spread Christianity and western education. Since the British government was mainly interested in economic activities, it only freed the slaves but left the burden of resettling them to the missionaries. The missionaries' engagement with freed slaves was driven by their desire to Christianize and "civilize" them. However, Sifuna (1990) reports that the missionaries pursued these goals in the guise that they wanted to "redeem and rehabilitate" freed slaves in order to recover the humanity that they had lost in the hands of the slave traders. Also, the missionaries guised that it was necessary that they "educate" freed slaves on how to survive in the comparatively alien conditions of the coast, because they could be recaptured and turned into slaves by their former oppressors if left on their own. In order to create a conducive environment within which they would be able to pursue their main goal of converting the freed slaves into Christianity, the missionaries started by establishing a station at Freretown. They embarked on a program of educating freed slaves on how to survive in their new environments. Also, they opened a school in the station where they taught freed slaves how to read and write in African languages to prepare them for later evangelical activities among fellow Africans. At the same time, the 
missionaries reached out to the local communities and encouraged them to embrace western education and Christianity.

The missionaries' efforts to spread Christianity and Western education were further boosted by the official establishment of British colonial rule in the $19^{\text {th }}$ century. Specifically, the colonial government provided funds and other indirect support that enabled the missionaries to expand their education facilities, especially opening new schools. Further, the construction of the Kenya-Uganda railway by the colonial government from 1895 helped the missionaries to establish new mission centers in the interior, and also attracted new missionary groups in Kenya (Eshiwani 1993).

Overall, the main goal of the missionaries was to train Africans how to read and write in their native languages so that they could understand the Bible and help in spreading Christianity to other Africans (Eshiwani 1993; Mutahi 1979; Sifuna 1990). However, these scholars report that since Africans had their own religious systems some of them resisted Christianization, prompting the missionaries to entice Africans using material gifts and privileges that were rewarding socioeconomically. Consequently, Africans started associating formal education with social mobility; hence many embraced it enthusiastically. According to Sifuna (1990), Africans even set up their own "bush" schools around the mission centers to meet the increasing demand for education among fellow Africans.

The missionaries on their part took advantage of the Africans' enthusiasm toward formal education to Christianize them. However, the missionaries were illprepared to meet such a high demand for formal education, especially because they lacked enough teachers, suitable curriculum, teaching materials and funds for use both 
in the central and African "bush" schools. Therefore, they hurriedly trained new teachers and dispatched them to the newly opened schools without a definite educational policy. As a result, the education provided to Africans during the early colonial period was very rudimentary. It was characterized by rote learning mainly emphasizing the acquisition of basic literacy and Christian values.

In spite of the poor quality of education, Sifuna observes that the missionaries continued opening more schools in the interior for various reasons. First, they wished to take advantage of the increased demand for education among Africans. Second, there was emerging fierce rivalry among the missionaries, especially the Catholics and Protestants, for supremacy in different areas of Kenya (Eshiwani 1993). Further, Eshiwani reports that the missionaries opened more schools in order to forestall the colonial government's efforts to play an active role in the education for Africans, mainly because the two had competing goals. According to Eshiwani (1993), the colonial government in turn argued that the fierce competition between the missionaries was negatively affecting the African in ways that undermined the main purposes for educating Africans. On the one hand, some missionaries were deliberately training Africans to mistrust rival missionary groups, hence setting a bad moral example to Africans. On the other hand, Africans had started embracing formal education for social mobility but the colonial government feared that this situation would lead to political agitation. As a result, the colonial government sought more and direct involvement in administering education for Africans to ensure that their own social and economic interests were being served (elaborated further below).

In addition to spreading Christianity, the missionaries had a further goal of “civilizing” Africans, i.e., inculcating western values. However, they argued that 
effective "civilization" would happen only if Africans stopped practicing their "heathen" cultural and religious practices (Sifuna 1990). Further, such civilization would best be achieved by removing the potential converts from their "pagan" environment and taking them to secluded areas. The two principles motivated the setting up of boarding schools in the mission stations. According to Eshiwani (1993: 17), "the missionaries used these schools and mission stations as centers for indoctrinating Africans into devaluing their culture and adopting the Western (European) way of life.”

The missionaries dubbed Christianization and Westernization as “enlightenment" and "civilization," two processes that facilitated the colonization of Africans by making them humble and faithful servants of the Europeans (Mwiria 1991). In this context, Mwiria (1991) regards early formal education in Kenya as “education for subordination." The subordination aspect, however, was not limited to Kenya but was part of the European colonial enterprise in Africa and beyond (Given 2004; Gosden 2004; Loren 2008). For example, Loren (2008: 79-80) reports that "control of the body, by self and others, was an inherent part of colonial rule...European crowns and their colonial officials sought to reestablish social order for their subjects through the creation of laws, edicts, and mandates when the structure of colonial order was threatened."

Even though the missionaries wanted to maintain full control of the education for Africans, Sifuna (1990) and Eshiwani (1993) report that they faced increased financial strains to keep up with the fierce competition among themselves on the one hand, and also to meet the increased demand for formal education among Africans on the other. Also, recall that colonial authorities had expressed their fears that the 
scramble for supremacy among the various missionary groups had negatively affected the initial goals for educating Africans. Therefore, the colonial government intervened in 1910 and urged the various missionary groups to cooperate and also partner in the administration of African education. However, Eshiwani (1993) reports that the colonial government only participated indirectly by providing funds and sometimes land until 1929, when it sought to be involved in the direct administration of "African" schools, i.e., schools for Africans. Next, I discuss British colonial education and associated goals.

\subsubsection{British colonial education in Kenya}

Initially, the colonial authorities were neither interested in spreading religion nor "civilizing" Africans. Instead, they specifically wanted Africans to gain basic education and literacy in order to provide cheap labor on the farms of white settlers and to work in junior positions to facilitate colonial administration (Eshiwani 1993). According to Eshiwani (1993: 15), "[b]oth the colonial administrators and the white settlers had a paternalistic attitude toward Africans and were therefore in agreement with the policy of rapid advance for the European and gradual advance for the African in education." The colonial administrative and educational policies were therefore aimed at producing Africans who would be of service to Europeans. In this regard, the colonial administrators employed indirect rule policy, which referred to the system of running the colonies by utilizing already existing socio-political systems to minimize administrative costs but maximize profits (Eshiwani 1993; Sifuna 1990).

In the context of education, indirect rule was christened "adaptation." According to Sifuna (1990: 47), it was called so because it emphasized "the 
adjustment of European institutions to local political and social organizations and the creation of a group of educated Africans, who at the same time would be rooted in their own culture." The main emphasis of the adaptation system was industrial training or technical education. The colonial authorities preferred technical education over the academic curriculum that was in use in Britain, to avoid empowering Africans, or equipping them with knowledge that would enable them to question colonial authority (Mwiria 1991). Specifically, the strong emphasis on technical and industrial education in Africa was motivated by the desire to combat political agitation as witnessed in British India, which would otherwise destabilize the British Empire (Gorman 1974; Mutahi 1979; Sifuna 1990).

Unlike the missionaries who used formal education as a bait to facilitate Christianization and "civilization" of Africans, the British colonial authorities aimed at adapting education to the local situation. According to Sifuna (1990: 48), colonial authorities stated that:

The school should be conducted in accordance with native customs in matters of dress and etiquette, in order that the pupils may not become denationalized or consider themselves a class apart...it was to train a generation able to achieve ideals of its own, without slavish imitation of Europeans....

The various restrictions led to formation of a racially segregated Kenyan society as follows: "Europeans" occupied the fertile rural lands and affluent neighborhoods in cities and "Asians" settled around the trade centers, especially along the KenyaUganda railway since many of them were engaged in business. Most "Africans," however, were restricted to the rural areas and the few who managed to come to the urban areas in search of manual jobs were segregated in low-income neighborhoods. The same racial segregation characterized education during the colonial period. This 
is well articulated by the Ominde Commission (1964, cited in Kang'ethe-Iraki

2010:1):

Education, like society, was stratified along racial lines, there existed an 'African education', a 'European Education', and an 'Asian Education'; three separate systems divided by rigid boundaries. This stratification was based on the colonialist's assertion that the mental development of the average African adult was equivalent to that of the average 7-8 year old European boy...AAfrican education therefore tended to be a hybrid, precariously hovering between a European model with a European subject matter, and an education deemed suitable to the place in colonial life considered 'appropriate' to the African population.

According to Mwiria (1991), the colonial authorities employed three main strategies to deny Africans opportunities to advance in formal education. These were: 1) unequal allocation of educational resources between the schools for Europeans, Asians and Africans in that order; 2) use of restrictive or punitive examinations to eliminate most Africans aiming at higher education; and 3) an oppressive ideology of the African school curriculum that emphasized technical education specifically designed to produce Africans of service to the Europeans. The curriculum was therefore designed to condition the minds of Africans to despise themselves, while praising the superiority of the religious and other cultural values of the colonizer. The same restrictions applied to the colonial language policy in which the colonial authorities deliberately denied Africans an opportunity to learn English, because they feared that Africans would become too educated to accept wage labor (Nabea 2009) ( I return to this further below.)

In summary, a critical look at the structure of formal education during the colonial period reveals that it was hegemonic; it aimed at subordinating and exploiting Africans and members of the other non-white races culturally and socioeconomically for the benefit of Europeans (Eshiwani 1993; Mwiria 1991; Sifuna 1990). Ultimately, the various educational practices socially constructed Kenyans and Africans in 
general as inferior physical and social beings in dire need of "help" and "redemption," hence justifying European religious and colonial activities (Mwiria 1991). But in what language(s) was formal education conducted during the colonial period owing to the competing sociopolitical and economic interests of the Europeans on the one hand, and the complex ethnolinguistic situation in Kenya on the other? I engage this question in the next section.

\subsubsection{Language policy during the colonial period}

According to Mutahi (1979), Kenya did not have a clear-cut language policy during the colonial period. Instead, there were multiple, inconsistent and sometimes contradictory language policies, reflecting the divergent educational goals and sociopolitical interests of the missionaries on the one hand, and the British colonial authorities on the other (also see Nabea 2009). I will only highlight some of the significant periods and associated historical events. I argue that the Kenya's colonial language policy was a site of struggle between groups with competing sociopolitical interests, leading to disconnects between theory (formulation of policies) and practice (implementation), a situation that has continued to the present.

\subsubsection{Colonial language policy between 1900-1929}

The period between 1900-1929 is significant because the British colonial authorities in Kenya, as noted earlier, were not directly involved in the administration of education. Instead, they concentrated on pursuing their administrative and economic interests. Therefore, their language policy required European colonial administrative officers to learn local languages so as to facilitate administrative efficiency, but Africans were not required to learn English (Eshiwani 1993; Gorman 
1974). The missionaries, on the other hand, assumed the entire burden of providing education to the Africans but could not agree among themselves on the appropriate language of instruction. For instance, since early missionary education was introduced along the Indian Ocean coast where Swahili was spoken natively and had already spread widely as a trade lingua franca, one group of missionaries comprised of early foreign scholars of Swahili such as Bishop Steere, Reverend Dr. Krapf and Father Sacleux, enthusiastically advocated for the use of Swahili in education and spreading Christianity (Gorman 1974).

However, Gorman (1974) observes that their successors did not share that enthusiasm but instead opposed the use of Swahili for proselytizing, because of its perceived association with the spirit of Islamization and the Arab slave trade. Instead, they favored the use of ethnic vernaculars for several reasons. First, they subscribed to the so-called Livingstonian principle that Africa would only be converted by Africans. In addition, these missionaries favored the use of ethnic vernaculars over Swahili, because they believed that it were speakers' first language rather than a lingua franca, which was best for reaching their innermost thoughts. Consequently, the missionaries embarked on translating parts of the Bible to be provided to the children in the mission schools.

From an educational point of view, Gorman (1974: 404) reports that these missionaries regarded "the home language as the best medium of instruction in a child's entry into school life and also for preserving whatever is good in native customs, hence preserving self respect." The various attitudes of the missionaries toward English, Swahili and ethnic languages were significant because they led to the 
initial steps in the formulation of the colonial language policy for African education (Gorman 1974). Specifically:

It was in the mission schools in all parts of Africa that the problem was posed whether the first steps in education should be taken in the local vernacular and it was the missionaries who were responsible for the earliest efforts at reducing the vernacular languages to written form. (Gorman 1974: 404-05)

In the context of this dissertation project, the missionaries' attitudes toward English, Swahili and ethnic languages are important because they reveal how differences in peoples' language ideology may affect both the formulation and implementation of language policies. In the following section, I will highlight some of the significant conferences or meetings where issues of the colonial language policy were discussed. I seek to demonstrate how the preference for one language variety over the others for a particular function such as education was driven by the sociopolitical and economic interests of the social group(s) in power rather than the educational desires or needs of the masses.

\subsubsection{The United Missionary Conference in Kenya, 1909}

According to Gorman (1974), owing to the complex ethnolinguistic situation in Kenya and the associated challenges of using multiple languages in education, the missionaries held a conference in Nairobi in 1909. The conference discussed at length questions about the use of English, Swahili and ethnic languages as media of education. However, different groups took different positions; hence the conference did not produce any particular language policy. Due to the lack of consensus, the various opposing groups of missionaries at the conference sought guidance from the colonial government about the language that was to be the lingua franca of the colony, to help them decide on the language they should use in African education. 
According to Mutahi (1979), the government did not provide an answer immediately. ${ }^{33}$ Instead, it formed a commission of inquiry in 1919 to consider educational policy in the protectorate. The various views discussed in the 1909 missionaries' conference were reiterated by this commission and discussed alongside those of other interest groups. I highlight some of the views discussed by the commission of 1919 below.

\subsubsection{The Commission on Education in the East Africa Protectorate, 1919}

According to Gorman (1974), the most telling arguments with regard to the teaching of English in African education came from the Headmaster of the Arab High School in Mombasa, who argued that Kenyans should be taught English to enable them take administrative jobs. Even though Gorman does not identify the ethnic/racial identity of said headmaster, and does not state explicitly that the headmaster was voicing the educational desires of Africans in this commission, it is clear that his language ideology resembled that of many Africans, who associated English with social mobility.

However, the views reiterated from the missionaries' conference favored the teaching of Swahili (Gorman 1974). First, the Allied Missionary Societies, an associated body of Protestant missionary societies, strongly supported the teaching of Swahili as opposed to English in the upper schools. Also, even though Allied Missionary Societies acknowledged the value of English in the higher training of the "natives," they strongly opposed the displacement of Swahili as the lingua franca of East Africa. Second, Bishop Willis from Uganda argued that Swahili should be the

\footnotetext{
${ }^{33}$ The government provided an answer 20 years later, in 1929.
} 
lingua franca of East Africa and early education should be done in Swahili with later transition to English. ${ }^{34}$ Third, the Roman Catholics, through Fr. J. Bergmans, opposed the teaching of English in elementary schools, arguing that Swahili should be used instead. Fourth, representatives from the colonial administration favored the teaching of Swahili and English but argued against the use of ethnic languages. The Provincial Commissioner for example, argued that the teaching of the "country" (ethnic) languages impedes trade, court work and retards advancement as a whole (Gorman 1974). But why were the various missionary groups and the colonial administration in favor of Swahili over the ethnic language varieties in elementary education, while emphasizing that English be introduced in the upper schools? ${ }^{35}$

According to Eshiwani (1993), those missionary groups and the colonial authorities who supported Swahili over the ethnic language varieties did so for economic reasons. Since Swahili was widely spread as a lingua franca, the cost of producing reading materials would be cheaper. Also, the administrative costs would be low because both the missionaries and colonial authorities would only be required to learn one language variety rather than the many ethnic vernaculars. With regard to English, I argue that it was unrealistic to say that it would be introduced in the upper schools because very few and sometimes none of the Africans advanced beyond grade three, where English-medium learning was to be introduced (Mutahi 1979; Mwiria 1991; Nabea 2009). Thus, the delayed teaching of English to Africans was a deliberate strategy of denying them the tool for social mobility.

\footnotetext{
${ }^{34}$ Gorman (1974) does not specify the missionary group that Bishop Willis represented.

${ }^{35}$ In this context, Swahili was NOT regarded as an "ethnic language." Instead, it was perceived as "foreign" because the variety that had spread into the Kenyan interior as a trade lingua franca was from Zanzibar, Tanzania rather than the local varieties spoken along the Kenyan coast (the politics of standardization of Swahili will be discussed in Chapter Four).
} 
Nabea (2009: 124) reports that English during this time "was to be taught to the Africans guardedly in order to ensure that the majority of them never acquired secondary and university education." In this regard, Mwiria (1991) reports that the missionaries subjected Africans to very selective examinations. Also, missionaries constantly encouraged Africans to start spreading Christianity to fellow Africans after grade three rather than pursuing further education for academic careers. This was in line with the missionaries' main goal of equipping Africans with basic literacy; just enough to enable them to read and understand the Bible, then embark on spreading Christianity further to fellow Africans. Accoding to Gorman (1974), the views of the said missionary groups and the colonial authorities were, however, contradicted by the Director of Education who argued that the teaching of ethnic languages was absolutely necessary while dismissing the teaching of Swahili as a waste of time. The Director's views were echoed in the conclusions of the commission, which advocated for the use of ethnic vernaculars in the initial stages of education with transition to English after the children had had considerable instruction in their ethnic languages. Also, the commission opposed the teaching of Swahili in favor of English, arguingthat "Swahili is a language that is foreign both to the employer and the employee. As a rule it is equally badly spoken by both...." (Gorman (1974: 406).

The Director's views, however, did not carry the day. Instead, Gorman (1974: 406) reports that "a minority report was appended, which was agreed to by four of the ten members of the commission, and which argued that the 'wholesale teaching of English...at present is impracticable.” The appended report instead favored Swahili on the grounds that it was the most easily acquired since it had already spread as a lingua franca. In spite of this, the minority report was in turn contradicted by the 
Director of Education who instructed that all the government-assisted schools should teach English where possible. This directive did not resonate well with all the missionary groups. As a result, the overall response was gradual until 1929 when the colonial government responded to the earlier question asked by the missionaries about the language that was to be the lingua franca of the colony, to help them decide on the language they should use in the education for Africans.

In summary, both the 1909 Missionary Conference and the Commission on Education in The East Africa Protectorate of 1919 demonstrate that language planning is a very political process; every stake holder advocates for a language policy that serves his/her interests. Such struggles greatly affect both the formulation and implementation of the affected society's language policies. In many cases, governments only implement parts of the proposed policies or shelve them altogether. It is also common for governments to revisit the shelved policies whenever they feel politically threatened but abandon them once the looming political agitation has been contained. Next, I focus on the colonial language policy between 1930-1949.

\subsubsection{Colonial language policy between 1930-1949}

This period is relevant because, though the colonial government declared that the lingua franca for East Africa was to be English in 1929, which was then to become the language of instruction in the schools for Africans, the policy was not implemented until 20 years later in 1949. Specifically, Mutahi (1979: 2), reports that the governor, Sir Macleay Grigg said, "every encouragement will be given to English, which must be the lingua franca of this colony. There shall be no bilingualism in our institutions or courts" (Mutahi 1979). Instead, the schools for Africans that had been 
using Swahili as the medium of instruction continued to use it. But why couldn't the colonial government implement its own policy statements? According to Mutahi (1979: 2), the settlers (who worked hand in hand with the colonial administers) felt that "English was a political tool that Africans had to be denied. To teach English to Africans [the settlers felt] would make them politically aware of the injustices done to them. For this reason, English was to be ruled out of African schools."

Similar contradictions were witnessed in subsequent policy statements during this period. For example, in an Educational Conference in Tanganyika Territory held in 1929, which brought together Directors of Education from the East and Central African Dependencies, the delegates recommended that they adopt Swahili as the lingua franca of East Africa (Gorman 1974). According to Gorman (1974), the conference resolved that Swahili be standardized on the basis of the Kiunguja (Zanzibar) variety and an Inter-Territorial Language Committee be formed to implement that resolution. The proposal to use Swahili sparked some controversy in Kenya and was further discussed in the Legislative Council in 1929. The Director of Education explained that the colonial government really wanted to make English the lingua franca of East Africa because it was aware that Africans had a strong desire to learn it. However, he regretted that the government could not fulfill the Africans' desire primarily because of lack of qualified teachers of English.

While it is possible that there were such practical challenges facing the teaching of English to Africans, I find the Director's characterizations of Swahili cited here below more relevant to this chapter because of their ideological richness. They further illuminate the political nature of the language planning process. The director of education described Swahili enthusiastically saying that: 
Swahili is a flexible language, an admirable educational medium through its flexibility. It is a rich language, enriched where it is itself deficient by one of the greatest of the oriental languages..., it is a literary language and... a common language already in common use in East Africa..., the majority of the missionary bodies in Kenya were in general agreement with the terms of the resolution [to use Swahili]. (Gorman 1974: 416-17)

Although it is not clear what the Director meant by "flexible,"or why Swahili would be more "flexible" than any other language (including English), my research findings indicate that many Kenyans do not perceive Standard Swahili as a flexible language. In fact, many of my informants (both teachers and students, in Nakuru and Mombasa) described it as a "rigid" and "boring" language because of its complex grammar, especially verb derivation and noun agreements, even though similar or even more complex grammatical forms exist both in other Bantu languages and in the Mombasa variety of Swahili. ${ }^{36}$ Also, the informants who claimed that Swahili is admirable referred to the variety of Swahili spoken in Mombasa but not the Standard.

From a political point of view, it should be noted that the proposal to standardize Swahili and make it the lingua franca of East Africa coincided with the plans of the British high colonial office in London to consolidate the whole of East Africa into one sphere of influence (Sifuna 1990). The choice of Swahili over English was therefore politically and economically motivated. Specifically, the colonial government preferred Swahili because it was more widespread as a trade lingua franca, hence its use would be more economical than English or local ethnic languages. Also, the British as noted earlier did not want to teach English to Africans in order to subordinate them. Nabea (2009: 124, quoting Mazrui and Mazrui (1996: 272) reports that, "[m]any European settlers regarded the teaching of the English

\footnotetext{
${ }^{36}$ Even though there are many varieties of Swahili spoken in Mombasa today, there is a particular variety called Kimvita, which is historically associated with Mombasa, and is also highly regarded by the locals, especially older Kenyans (Wald 1985).
} 
language to the "natives" as potentially a subversive force. Social distance between master and subject had to be maintained partly through linguistic distance."

The delayed teaching of English in African schools continued up to the end of the Second World War, which was a significant political turning point in Africa. According to Sifuna (1990: 150), "[t]he war aroused nationalist consciousness and increased demands for the expansion of educational opportunities at all levels of the school system" (also see Eshiwani 1993). Two main categories of Africans were involved. The first group was comprised of the few educated Africans who had gone to secondary school and beyond. The harsh and frustrating experience they went through while pursuing education had made them aware of the poor quality of education provided to most Africans and other socioeconomic injustices characteristic of racially segregated Kenya. They therefore sought to provide a new strategy to political leadership, which not only aimed at rectifying the said socioeconomic and political injustices but also to overthrow the colonial system (Mazrui and Mazrui 1995). The coming together of these educated Africans marked the birth of the black elite in Kenya. For instance, Sifuna (1990: 144) explains that, since these educated Africans were able to speak, read and write in English:

They became the spokesmen of their tribal societies through a process of nationalism whose main unifying force was the presence of a colonial power. They were often approached by the elders to stand for election in local administration. The elite believed that their representation at the national level was a right and not an ordained duty.

These educated Africans formed political associations such as the Kikuyu Central Association and Young Kavirondo Association. They also engaged the colonial authorities in round table negotiations, specifically pushing them to relinquish land 
and power in order to pave way for Kenya's socioeconomic and political independence.

The other group of Africans involved in nationalist activism was comprised of the young men who had gone to fight alongside the British army in the Second World War. According to Sifuna (1990: 58), "[t]he collective wartime experience of the Africans proved to be of great significance during the post-war period. They discovered European vulnerability, and their return after the war contributed greatly to the 'African Awakening." These Africans used the same Swahili (regional lingua franca) that had been used to facilitate colonial administration to unify and mobilize fellow Africans to revolt against the colonial government. In other words, a new wave of Kenyan nationalism that the British had all along tried to suppress was gathering momentum and was solidifying around African languages, especially Swahili (Nabea 2009).

The British colonial government in turn hurriedly initiated the replacement of Swahili with English as the medium of educational instruction. It formed a commission of inquiry in 1943, led by Bishop. L. J. Beecher, to audit and report on the status of African education. According to Mutahi (1979: 4), the Beecher report of 1943 emphatically recommended the teaching of ethnic languages and that "English should take the place of Swahili as the colony's lingua franca in as short time as practicable." While the colonial authorities argued that the teaching of Swahili as a lingua franca impeded the learning of both ethnic languages and English, it is clear from the foregoing discussion that this recommendation was informed by the colonial divide-and-rule policy. 
However, Mutahi (1979) and Gorman (1974) report that the teaching of English in African schools was not implemented. Instead, very stringent conditions were put in place with regard to the teaching of English for non-European schools. These conditions are well outlined by Gorman (974: 433) as follows:

For any primary school to teach English there had to be suitably qualified staff. Second, syllabuses and the schemes of work must be submitted to the Education department for approval. Third, children who had been taught the groundwork of English at the primary stage must be able to continue from that stage to the intermediate level. Five, English should be taught in a primary school only with the express permission of the Education Department on the recommendation of the Provincial Education Officer, and that authority should be given for the withdrawal of the grant-in-aid to primary schools where English was taught without permission.

As a result, African schools continued using Swahili and vernaculars until 1949 when the colonial government ordered the Beecher commission to do another audit report. The Beecher report of 1949 recommended that more intermediate schools (grade 5-8) be built and should use English as the medium of instruction while the mother tongues should be used in the lower classes. The colonial government adopted the Beecher report in 1950 and the teaching of English in African schools started immediately.

The fact that it took the government two decades to implement the teaching of English in African schools further points to its general lack of political obligation to implement the recommended language policies in Kenya unless they served its political interests. But what was unique about the Beecher report of 1949? That is, if the colonial government had previously attributed the delay in the teaching of English in African schools to a lack of qualified teachers, what changed in the year 1950? I engage these questions in the following section.

\subsubsection{Colonial language policy between 1950-1962}

The period around 1950 witnessed increased pressure across Africa for political independence; hence the British colonial authorities started realizing that 
colonialism in Kenya was coming to an end. However, since the British government still wanted to maintain its political and economic influence in Kenya after independence, it had to adjust its policies though in a strategic way. For example, Mutahi (1979: 4) observes that "[t]he colonial policies and attitudes had to change so as to make it possible for the imperialists to recruit the future leaders who would safeguard their interests after Uhuru. ${ }^{37}$ In other words, the British colonial authorities wanted to promote the teaching of English in order to lay the foundations of neocolonialism, before Kenya gained its independence in 1963. This is what makes the Beecher report of 1949 stand out over previous educational reports.

The adoption of the 1949 Beecher report led to very drastic changes in the education structure and also language policy for African education. First, the education system was restructured from 6-2-4 (six years of primary, two for intermediate and four years for secondary education) to 4-4-4 (four years each for the three levels). Second, there were deliberate efforts to discourage the use of Kiswahili on the one hand, while promoting the teaching of English on the other. For example, from 1953 onwards, English was made the compulsory medium of instruction in the examination that was done at the eighth (final) year of primary education (Gorman 1974; Mutahi 1979). Further, Mutahi (1979) reports that the stringent conditions that were to be met earlier before any school for non-Europeans could be allowed to teach English no longer applied from 1953 onwards.

As noted above, the wave of political independence that was looming in Africa had prompted the colonial authorities to change their policies and attitudes toward Africans. According to Mutahi (1979), the system was

\footnotetext{
${ }^{37}$ Uhuru means independence/freedom in Swahili
} 
changing from when the colonial masters regarded Africans as sources of cheap labor to future political and economic "partners." What is most important in the context of language policy, however, is that the British were now contradicting their previous language ideology. For example, the colonial authorities were initially against teaching English to Africans and were also opposed to the idea of Africans imitating the Europeans. Surprisingly, the British now wanted Africans to learn and embrace English and associated identities from 1950. Specifically, Mutahi (1979: 5) reports that the governor said, "[w]hat we have set out our hands to here is the establishment of a civilized state in which the values and the standards are to be the values and the standards of Britain, in which everyone, whatever his origins, has an interest and a part...." The new British civilization policy further cemented the emerging cadre of black elite who were by then warming up for power and other positions occupied by the colonial authorities.

Unlike in the period between 1900-1949, the colonial government was very consistent in implementing its policies between 1950-1963. The Beecher report of 1949 was implemented comprehensively. For instance, it led to the introduction of the English Medium Programme in 1958, in which English was adopted as the official medium of instruction right from the first class of the primary education. At the same time, the value of English was emphasized over Swahili and ethnic vernaculars. According to Eshiwani (1993), the English Medium Approach was later renamed "The New Primary Approach" to conceal the fact that it was intended to privilege the teaching of English over the other language varieties (also see Nabea 2009). 
Despite renaming the program, the education goals never changed. In fact, Mutahi (1979) argues that The New Primary Approach aimed at entrenching cultural imperialism, whereby the values and the standards of Britain were to be made the values and the standards of independent Kenya. By 1962, the New Primary Approach was in full operation; hence Kenya inherited colonial educational structures including the language policy at independence in 1963. The black elite took over the positions and neighborhoods formerly occupied by the colonial authorities and from there on, Kenya transitioned from a racially to socioeconomically segregated society.

In summary, the discussion so far has traced the trajectory of Kenya's formal education and associated language policy throughout the colonial period (1900-1963). I have demonstrated that language and education are intrinsically intertwined and also related to other aspects of peoples' lives such as identity, history and traditions. Thus, decisions made about language and education are political. The colonial authorities' language ideology with regard to English in Kenya is a perfect example of the political nature of the language planning process. In the initial years of the colonial rule, the government deliberately denied or delayed the teaching of English to Africans, citing practical challenges. However, it intensively promoted the teaching of English to Africans in the period surrounding independence. While it may appear on the surface as if the colonial authorities had given in to the demands of the Africans, a critical look at the change of policy from withholding English from the Africans (to suppress political agitation) to bestowing it (as 
an instrument of civilization)--was a survival strategy for the British colonial authorities, to remain politically and economically influential even after Kenya gained political independence.

Further, the discriminate teaching of English and its association with better job opportunities during the colonial period endowed it with some social value over Swahili and other ethnic languages. Over time, Africans started associating English with social mobility; hence they enthusiastically pursued it while despising Swahili and the ethnic languages. But what happened to Kenya's language policy after independence? What attitudes do Kenyans have toward the various languages spoken in Kenya today including Standard Swahili and English? I engage these questions in the next section by tracing the Kenya's national language policy from 1963 to the present.

\subsubsection{Kenya's language policy from 1963 to date}

Despite the fact that Kenya inherited colonial structures in 1963, it had its own aspirations to meet as an independent state. These included national unity, restoration and reinforcement of African cultural identity and reduction of foreign economic assistance (Sifuna 1990). Also, the government faced myriad challenges from the massive rural-urban migration of Africans in search of employment and better life opportunities. The government therefore had to expand its basic infrastructural facilities in education, health and housing. Further, the fluid movement of people created complex ethnolinguistic situations mainly in the urban areas. Therefore, there was a need for the government to rethink the country's educational and language 
policies. The main sources of language policies in independent Kenya are: 1) educational commissions of inquiry; 2) "roadside" or impromptu declarations by the presidents and other politicians; and 3) constitutional amendments. For the purposes of this chapter, I will discuss only a few of these sources starting with the educational commissions of inquiry.

\subsubsection{Educational commissions of inquiry from 1963 to the present}

\subsubsection{The Ominde Commission}

In December 1963, the government ordered an audit report to survey the status of education and advise on the formulation and implementation of national policies for education (King'ei 2001; Mutahi 1979; Sifuna 1990). These scholars report that the Ominde Commission sought to reform colonial education. It reported that the prevailing conditions in Kenya were totally different from the colonial period suggesting that Kenya's independence marked the birth of a new nation. Therefore, the Commission advised that education in independent Kenya had the task of uniting the different racial and ethnic groups that make up the nation. With regard to language policy, the Commission recommended that English be adopted universally in education as the viable medium of instruction. Also, Swahili should be introduced as a compulsory subject from the first year in primary education, and a department of Swahili should be started at the University of Nairobi to promote the language. But what informed the Ominde Commission's foregrounding of English in education, a language only spoken by the minority, over the ethnic 
vernaculars and the widespread Swahili? According to King'ei (2001: 39), the commission argued that:

English provides a better medium for learning languages and literacy than the vernaculars. That English would provide a more systematic and quicker development in all other subjects of study. That the foundation laid in the first formative years of schooling would be more scientifically conceived and solidified if offered in English as opposed to the vernaculars. That the transition from vernaculars to English medium was difficult and unnecessary.

While the Ominde Commission was tasked with reforming the colonial education system that Kenya inherited at independence, a critical look at the policy statements above reveals that the Commission's language ideology was closely related to the very colonial ideology it was seeking to reform. Broadly speaking, such language ideology has led to preferential treatment of the former colonial languages in the domains of power such as in courts, or in specialized knowledge such as science, over local African languages in postcolonial Africa (Adegbija 1994; Makalela 2005). In this regard, the Ominde Commission's association of English with science socially constructs it as more sophisticated than African languages. However, it should be noted that despite constructing English that way, it is not true that some language varieties are intrinsically incapable of being used for one function but not in others (Lippi-Green 2012). Instead, Lippi-Green (2012) observes that functional differences between language varieties, e.g., English versus African ethnic vernaculars, arise when one language variety is picked and developed over others for social and political reasons.

From a pedagogical point of view, the following claims made by the Ominde commission are not true: 1) that English is a better medium for 
learning languages and literacy and would make education systematic, and 2) transition from vernacular to English is difficult and unnecessary. In fact, educational research has confirmed that there are many pedagogical advantages of building upon the leaner's home language when learning the standard or authorized language (Powers 1998; Rickford 1999; Siegel 1999).

As noted earlier, such contradictions between official language policies and research into how languages actually function in communities, occur mainly because language planning at the national level is driven by the interests of the ruling class, rather than the communicative needs or socioeconomic desires of the masses. It is therefore common for governments in post-colonial Africa to either implement policy recommendations selectively or shelve them altogether. For example, King'ei (2001) reports that the government adopted the Ominde report report in 1964 and implemented the policy recommendation about English immediately, but delayed those about Swahili. Specifically, the report had recommended that a department of Swahili be set up at the University of Nairobi but this was not done until 1969. Also, the report had recommended that Swahili be introduced as a compulsory subject from the first year in primary education but this was not implemented until 1985. The preferential treatment of English over Swahili in the implementation of the policy recommendations of the Ominde Commission marked the beginning of the disconnect between theory and practice in the post-colonial period. Next, I will focus on the Wamalwa report (1972), which focused on the state of Kenya's human resources but made relevant recommendations related to the national language policy. 


\subsubsection{The Wamalwa Report (1972)}

As noted earlier, the government at independence also aspired to create more job opportunities and enhance national unity. Therefore, it ordered an audit report in 1972 to survey the state of the country's human resources and come up with appropriate policies. According to King'ei (2001), the Wamalwa Report advised that:

Strong emphasis should be placed on programmes teaching foreign languages such as French and German in order to promote international trade, tourism and diplomacy. That since most civil servants did not have a good command of Swahili, the national language, special in-service courses should be mounted at all training colleges for staff.

Even though the two recommendations were fully implemented, there was a disconnect between the policy and people's actual language use at two levels. For example, King'ei (2001) reports that government officials continued addressing the public in English because of the negative attitudes they had toward Kiswahili and ethnic vernaculars. ${ }^{38}$ Also, even though degree programs offering foreign languages such as German and French were introduced, student enrollment rates were generally low as compared to those in Kiswahili and English. From my own observations, I argue that the low enrollments may be attributed to lack of career opportunities associated with these languages in the immediate job markets. However, there is need for indepth research to find out the attitudes that Kenyans have toward foreign languages such as French and German.

\footnotetext{
${ }^{38}$ King'ei (2001) does not state explicitly the negative attitudes that government officials held toward Swahili and ethnic language varieties.
} 
Another report that further reveals the disconnect between theory and practice in Kenya's national language policy was done in 1976 by the Gachathi commission. I outline some of its recommendations here below.

\subsubsection{The Gacathi Report (1976)}

The Gachathi commission of 1976 was tasked with reviewing the educational policies and objectives in Kenya. While it made numerous recommendations aimed at improving education, it contradicted itself in some areas and also contradicted the Ominde Report of 1964. According to King'ei (2001: 40), the Gachathi commission recommended that "[t]he medium of instruction in primary one to three be the dominant language of the school's catchment area." This development reversed an earlier recommendation by the Ominde Report (1964) that English be the universal medium of instruction in all schools, which destabilized learning at the lower levels with regard to production of learning and teaching materials. The recommendation also introduced a problematic notion of "catchment area" because most of the residential neighborhoods in independent Kenya had become more cosmopolitan unlike during the colonial period. It would therefore be hard to pick one dominant ethnic language for use in education without disadvantaging some Kenyans in the process.

The Gachathi report (1976) also recommended that "Kiswahili be introduced as a subject only at Primary Three or whenever English was adopted as the medium of instruction" (King'ei 2001: 40). This recommendation contradicts the Ominde commission, which had earlier recommended that Swahili be introduced as a compulsory subject from grade 
one. Lastly, the Gachathi commission contradicted itself with regard to the use of the dominant language of the school's catchment area from primary one to three when it recommended that "[t]he introduction of English as the medium of instruction to take over from mother tongues as soon as possible" (King'ei 2001: 41). As noted earlier, the recommendation to make Swahili a compulsory subject was not implemented until 1985.

There have been other educational commissions of inquiry such as the Mackay (1981) and Kamunge (2000), most of which echoed the need to use the dominant language in the school's catchment area in the first three years of education. They also recommended that both Swahili and English be taught as compulsory and exam subjects in both primary and secondary schools. All these educational reports culminated to the current national language policy which stipulates that: 1) all elementary schools in the ethnically homogenous areas to use the dominant ethnic language while those in the cosmopolitan areas are to use Standard Swahili for the first three years of education; 2) from grade four onwards, all elementary schools are supposed to then switch to English-medium instruction in all other subjects apart from Swahili (Bunyi 2005; Eshiwani 1993; National Constitution 2010). However, as mentioned at the beginning of this chapter, neither teachers nor students adhere to the said language policy. In the next section, I outline the relevant roadside declarations related to Kenya's national language policy. 


\subsection{2. "Roadside" or impromptu declarations by politicians}

The term "Roadside declarations" in Kenya is used casually to refer to statements about issues of national interest that the president and other senior government officials make in unofficial fora, during political rallies or when addressing the National Assembly. Most of these statements either take many years to be revisited or are never implemented at all. The two most relevant declarations were made by Jomo Kenyatta, the first president of Kenya in 1964 and 1969, about the use of Swahili as the official language of the National Assembly. According to Mutahi (1979), Jomo Kenyatta made a statement casually when addressing the national assembly in 1964, which was to become the future national language policy. The president said in Swahili:

Bwana Spika, mimi nataka kusema maneno kidogo kwa Kiswahili kwa sababu natumaini kwamba wakati si mrefu katika nyumba hii yetu tutaweza kuzungumza Kiswahili ambacho ni lugha yetu. (Mutahi 1979: 7)

Mr. Speaker, I want to say a few words in Swahili because I believe that in this assembly, we will soon be able to use Swahili, which is our language. (Mutahi 1979: 7)

Since the president's statement was not followed by a systematic program of implementation, many Kenyans could have dismissed it as not a policy. However, Mutahi (1979: 7) reports how Dr. Waiyaki, a government minister, surprised the country when he stated in his speech while addressing the National Assembly in 1965 "[t]hat Kiswahili was the national language of Kenya and that no debate was needed on the subject."

Despite making it official, however, the government did not set up a program to facilitate the implementation of the said policy until 1974 when the issue was revisited. According to Mutahi (1979), the governing council of the 
ruling party, KANU (Kenya African National Union) decided to make Swahili the language of the National Assembly. The proposal was discussed and passed in parliament. These developments necessitated constitutional changes to include a clause that parliamentary debates would be conducted in Swahili. However, this was contradicted by another constitutional amendment that required that all government documents, including the bills discussed in parliament, to be written in English. The two new clauses further contradicted another clause about minimum competence in English for parliamentary aspirants. Mutahi (1979: 8) reports that the constitution required that all parliamentary aspirants to "be able to read the English language well enough to take an active part in the proceedings of the National Assembly." This recommendation raises the question why the constitution would require parliamentary aspirants to be competent in English, while parliamentary debates are to be conducted in Swahili.

Owing to the general disregard that Kenyans had toward Swahili and ethnic languages, and the inconsistent implementation of language policies in the schools, very few members of the elite had the required competence in Swahili to be eligible to vie for a parliamentary seat. Therefore, I argue that the requirement to have parliamentary aspirants demonstrate certain competence in English in order to be eligible to vie for a parliamentary election was a strategy that the elite employed in order to reduce political competition. This is because only a very small number of Kenyans could meet the required English competence in 1974 on the one hand, and because many of the parliamentarians strongly opposed the use of Swahili once they 
got to parliament on the other. For example, Charles Njonjo, the then Attorney General argued that, "Kiswahili was an Arabic language and if all foreign languages were to be done away with, then it should be among them. Also, nearly every MP has his own way of speaking Swahili and to use it here would make the House like the Tower of Babel where nobody would understand whatever the other said" (Standard Newspaper, August $30^{\text {th }} 2010$ ).

Njonjo's characterization of Swahili as an Arabic language with an implication that it is foreign is paradoxical because English, his preferred alternative, is also foreign. Similarly, his argument that parliamentarians spoke different varieties of Swahili is also paradoxical because it assumes that English is homogenous, which is not the case. Further, his characterization of Swahili as an Arabic language is contestable-some people claim it is a Bantu language-- but details of this problematic and its relevance to this dissertation will be discussed in the next chapter. What is important at this point is that Njonjo's language ideology contributes to the characteristic disconnect between theory and practice with regard to Kenya's national language policy.

From the foregoing discussion, I argue that the National Constitution has contributed significantly to the pervasive disconnects between the national language policy and peoples' actual language use in Kenya since 1963. Despite the fact that the new Constitution (2010) has brought a wave of sociopolitical reforms in Kenya that had not been witnessed before, it still privileges English over Swahili in significant ways. For instance, even though both languages are now official, the new constitution states that "[i]f there is a conflict between different language versions of this 
Constitution, the English language version prevails" (National Constitution 2010: 159). That statement accords English more legitimacy as an official language than Standard Swahili, a situation that adds to the already ambiguous status of Standard Swahili in Kenya.

In a similar vein, the continued privileging of English over the other language varieties not only helps to maintain the status quo, but also disadvantages the Kenyans with lower levels of education. Consequently, English has retained its colonial, elitist and exclusionist attributes, fifty years after independence. Further, even though the National Constitution promises to promote and protect linguistic diversity in Kenya, the government discourages the use of ethnic languages in certain domains and public spaces. For example, Samper (2002) reports that the government constantly censures the use of ethnic languages such as in the media, arguing that the promotion of ethnic languages amounts to promotion of "tribalism." More recently, a motion seeking to ban the use of ethnic languages in public offices was debated and passed in parliament (Daily Nation Newspaper, June $8^{\text {th }}$ 2011). The proponents argued that the use of ethnic languages in public offices fueled tribalism, which is a threat to national unity and an impediment to both patriotism and economic growth. Also, the proponents added that the use of ethnic languages in public offices excludes some people from communication and also compromises service delivery.

Further, the government discourages and sometimes condemns the use of nonstandard language varieties such as Sheng arguing that they spoil the "pure" Standard Swahili and English (Momanyi 2009). For example, the minister of education attributed the candidates' poor performance in Swahili national exams to their use of Sheng (Standard Newspaper, $29^{\text {th }}$ December 2011). Also, Githiora (2002) 
reports that many figures of authority regard language varieties such as Sheng as "corrupted" forms of Standard Swahili, which are used by "dodgy" people. These attitudes are similar to the ones expressed by the MPs earlier in the chapter that idiosyncratic varieties of Swahili are incomprehensible. These attitudes were an earlier manifestation of the "purist" language ideology surrounding Standard Swahili, which is not true because Standard Swahili draws vocabulary from various languages. This is well demonstrated in the sentence used by Chachage (2004) below. (I use bold to mark borrowed roots): "nilipobatizwa nilienda shuleni kuhesabiwa kisha nikala chakula mezani na kupanda gari kwenda hotelini [When I was baptized, I went to school to be counted then ate food at the table and got into a car]." According to Chachage, the sentence is "made up of words from at least seven languages namely: Baptise (English/Greek), Schule (German), Hisab (Arabic), Meza (Portuguese), Gari (Hindi) and Hotel (English/French)." 39

In conclusion, this chapter has traced the development of formal education and the associated language policy from the colonial period to the present. It has established that both were introduced by the early Christian missionaries who were later joined by the British colonial administrators. The missionaries introduced the notions of "ethnic language" and "tribe" to facilitate the codification of African languages to be used in translating the Bible, and spreading Christianity among Africans. However, I have argued that these two notions are problematic constructs that do not fit well with the reality of language and ethnic identity in multilingual Kenya. Also, some of the examples demonstrated that the distinction between "dialect" and "language" is political. For example, the Bukusu, Nyole and Idakho

${ }^{39}$ Retrieved from: http://www.pambazuka.org/en/category/comment/64058 
which are regarded as Luhya dialects are coded as distinct languages in the Ethnologue map. Therefore, I argued that the various categories of identity generated from the correlation between "one language" and "one culture" or "tribe" do not reflect Kenya's ethnolinguistic diversity in full. This disconnect suggests that any attempt to study social groups should seek to know how they define themselves, rather than fitting them into the already existing criteria. The racial categories used during the colonial period were also problematic because they were used exclusively. However, like the problematic ethnic labels, the racial categories were convenient to the colonial authorities in line with the British divide-and-rule policy.

The chapter also demonstrated that language policies in multilingual societies such as Kenya are usually driven by the sociopolitical and economic interests of the ruling class. Thus, I echo Mutahi's (1979) assertion at the beginning of this chapter that the government only responds to issues of language when the political system is threatened. However, it immediately forgets these issues as soon as the political "dust" settles. During the colonial period in Kenya, for example, the colonial government favored the use of Swahili over English and ethnic languages in order to reduce administrative costs, but replaced Swahili with English immediately after it realized that Swahili was uniting Kenyans against it. Similarly, the colonial government had all along denied Africans the opportunity to study English for fear of political agitation, but it intensively promoted the learning of English in African schools when it realized that Kenya's independence was inevitable. I argued that this was a strategy to remain politically and economically influential in post-colonial Kenya. 
From the recent past, it has become common for the Minister of Education and other figures of authority in Kenya to attribute students' poor performance in Standard Swahili and English to Sheng when announcing results of the national exams. Also, the government constantly censures the use of ethnic languages, especially in the period surrounding elections arguing that they promote ethnic animosity. While it is true that ethnic languages are sometimes used to instigate violence like in the period surrounding the 2007 contested elections, it is striking that such concerns from the government vanish as soon as the general elections are over. The government then reverts to use of English, its "comfort zone," hence language issues that affect the masses or relate to their sociopolitical or economic desires are rarely addressed. Even when the relevant language policies are formulated by the commissions of inquiry, they are rarely implemented. In the long run, the national language policy only exists in theory. In the next chapter, I focus on how the current national language policy in Kenya articulates with peoples' actual language use patterns in their quest for national identity. 


\section{Chapter Four}

\subsection{Sheng, Ethnicity and Politics of National Identity in Kenya}

'Sheng' has rapidly evolved in Kenya into a way of life, a culture, a standpoint, a nation...The 'Queen's English' and 'Kiswahili Sanifu' [Standard Swahili] of Kenya's 'forefathers', have [...] unfortunately failed terribly in forging a just nation in which all and sundry can live, thrive and prosper, regardless of age, religion, tribe, province or culture. It is out of these circumstances that the 'Sheng Nation' can be said to have evolved....The 'Sheng Nation' [...] is the basis on which millions of Kenyans [...] reach out to each other and the wider world, in the quest for identity, recognition and the right to develop our beautiful vast and blessed land, as we deem fit. (The Sheng Nation, 2010) ${ }^{40}$

This chapter focuses on the contested status of Standard Swahili as the national language of Kenya. As noted in the previous chapter, Kenya at independence inherited a racially segregated society, hence the government sought to unite the different racial and ethnic groups into one nation, and in the process cultivate a sense of national identity. The government also aimed at reinforcing African cultural identity by localizing the educational curriculum with regard to content and media of instruction. However, the colonial structures that Kenya inherited at independence socially constructed ethnic languages as inappropriate for educational use, and as impediments to national unity (Harries 1976).

The same language attitudes continued after independence since the Ominde Commission (1964), which was tasked with reviewing the existing educational structures, reported that ethnic languages were ill-adapted for use in education, and developing them would promote sectionalism rather than

\footnotetext{
40 The Sheng Nation is an online blog run by young Kenyans who associate with Sheng. Their attitudes toward Sheng exemplify how young people have embraced nonstandard language varieties as an alternative mode of communication, and as tools for negotiating desired social identities. (See <http://www.kenyanlist.com/kls-listing-show.php?id=38090>)
} 
national integration (Wa'Njoroge 1986). At the same time, the government could not choose English, Arabic, or Gujarati, because they were associated with foreigners, and colonial oppressive and exclusivist ideology. However, the government acknowledged that Swahili had a unifying role in East Africa, and suggested that the language be taught as a compulsory subject in Kenya's schools (Mazrui and Mazrui1995). Even if this policy was not implemented immediately, the government announced in the same year that Standard Swahili would be the national language of Kenya, arguing that it was widespread as a lingua franca, relatively neutral from an ethnic point of view, and had already been standardized (Harries 1976; Wa' Njoroge 1986).

Standard Swahili is based on the Kiunguja variety which is spoken in Zanzibar, Tanzania. However, its elevation to the status of national language in Kenya has always been contested by speakers of the local coastal varieties of Swahili, especially Kimvita, which is associated with Mombasa (Chimerah 1998; Whiteley 1993). Speakers of Kimvita argue that their language is better fitted as Kenya's national language than the current "standard," and have even called for re-standardization of Swahili based on Kimvita (Harris 1976; Khalidi 1977; Mbaabu 1985; Mkude 1984). In addition, some young Kenyans, as indicated in the opening quotation, have embraced nonstandard language varieties like Sheng over Standard Swahili, with some envisioning Sheng as Kenya's future national language (Daily Nation Newspaper, February $21^{\text {st }}$ 2012; Sheng Nation 2010). Further, some scholars have reported that it is Sheng rather than Standard Swahili that serves as a lingua franca among many young Kenyans in the cosmopolitan areas (Migunda-Attyang 2007). That is, 
Sheng seems to be fulfilling the function of the preferred language for interethnic communication that was originally intended for Standard Swahili (see section 4.5.0).

The contested status of Standard Swahili therefore lends itself to the following questions:

1) Why does the government perceive Standard Swahili as "ethnically neutral?"

2) What informs the claim by speakers of Kimvita that their language is better suited to be Kenya's national language than the current standard?

3) What credence should be given to Sheng speakers' claim that it is Kenya's future national language?

4) Owing to the heterogeneous nature of Kenyan youth, what attitudes do speakers of other competing nonstandard language varieties such as lugha ya mtaa in Mombasa express toward Standard Swahili as a national language?

I will address these questions in the remainder of this chapter, beginning with the perceived neutrality of Standard Swahili as a national language in Kenya.

\subsubsection{Perceived "ethnic neutrality" of Standard Swahili as a national language}

The notion of "ethnic languages," as mentioned earlier, is a problematic construct that does not fit well in all cases, even though it is used in Kenya to refer to the languages associated with the approximately 42 officially identified ethnic groups (Ogechi 2002). From a historical point of view, Wa'Njoroge (1986: 346) reports that, “...the post-colonial period brought out in the open rivalries based on the linguistic affiliations, and the development of one or a few local languages was seen as an attempt to dominate the other ethnic groups." Even at the present, Kenya is politically 
and economically dominated by a few major ethnic communities such as Kikuyu, Luo, Kalenjin and Luhya. This dominance raises acute inter-ethnic rivalry in matters of national interest. As a result, national politics such as appointment of government officials and distribution of national resources are often driven by ethnicity. Thus, many Kenyans vote along ethnic lines during general elections, because they expect political rewards from the winning presidential candidate in return.

Such politicization of ethnicity in matters of national interest arouses ethnic consciousness. For example, following the introduction of multi-party politics in 1991, speakers of Suba, previously classified as a dialect of Dholuo sought political recognition as a distinct ethnic group and their wish was granted (Ogechi 2002). ${ }^{41}$ This led to an increase in the officially recognized number of ethnic groups, indicating the power of the discourse of language and culture in claiming position or relevance in sociopolitical matters (Handler 1994). Also, the recognition of Suba as a distinct ethnic group indicates that the government in post-colonial Kenya subscribes to the colonial ideology that every ethnic group has its own language. Such language ideology makes it difficult for the government to adopt one of the local ethnic languages for national use without arousing ethnic antagonism.

In multilingual contexts similar to that of Kenya, policy makers often resort to a compromise language, which is often foreign or a local language

\footnotetext{
${ }^{41}$ There are many sociopolitical advantages of being recognized as an ethnic group in Kenya. Since Kenyans in general usually vote along ethnic lines, distribution of political power and national resources is also done along ethnic lines. As such, a group with ethnic autonomy can demand and lobby the government to address its interests. In this context, the notion of ethnic balance in the government has always been contested since every ethnic community wants to be represented.
} 
not associated with a dominant group or one of the competing social groups (Brock-Utne 2005; Makalela 2005; Wa’Njoroge 1986). As such, despite the fact that choice of foreign languages as official or national languages is often perceived negatively by citizens of the affected countries, they are usually preferred because they are perceived as relatively more neutral than any of the local languages. In Kenya, the variety of Swahili that was adopted as the national language is spoken in Zanzibar, Tanzania, where it is not associated with any single ethnic unit (Harries 1976; Whiteley 1993). Also, the two scholars observe that the total population of Kenyans that speaks a variety of Swahili as mother tongue is relatively small, and this group is not socially or economically powerful.

In addition, there exist historical tensions between speakers of the upcountry and coastal varieties of Swahili, which improved the chances of Standard Swahili being accepted by the rest of the population in Kenya (Mazrui and Mazrui 1995; Whiteley 1993). Specifically, Whiteley (1993: 65) states that, "[t]hough Kenya has a long coastline along which Swahili is spoken as a first language, this coastal form of Swahili has never been accepted up-country; while the various upcountry varieties of Swahili...have never been adopted at the coast." Also, the locus of political power in Kenya is in Nairobi and not along the coast where there are highly regarded local varieties of Swahili (Eastman 1984). ${ }^{42}$ The various factors therefore informed the government's perception of Standard Swahili as more neutral than the

\footnotetext{
${ }^{42}$ However, Nairobi as a political locus of Kenya has recently been perceived as a challenge to the spread of Standard Swahili because it is the perceived origin of Sheng and Nairobi Swahili, which are more popular among many young Kenyans.
} 
local varieties of Swahili, i.e., both coastal varieties that were their speakers' first language and pidginized "upcountry" varieties used as contact languages. Further, the contested ethnicity of the Swahili people in East Africa also contributed to the choice of Standard Swahili as the national language of Kenya (Eastman 1971; Mbaabu 1985; Wald 1973, 1985). Some scholars argue that there is no "ethnic group" called Swahili while others maintain that there is. For example, Senkoro claims that Swahili refers to citizens of East and Central Africa, and this category is not limited to the people who live along the coast (Mazrui and Mazrui 1995). In this context, Mazrui and Mazrui (1995: 86) argue that the "Swahili people are seen as having been subsumed under a more general, trans-national identity on the basis of the long-term cultural impact of Swahili." Closely related to the transnational identity of the Swahili is that the majority of the people to whom the label 'Swahili' applies are from diverse racial and ethnic backgrounds.

From another point of view, the Swahili identity is very fluid. According to Eastman (1971: 229) "[w]hile a Swahili must have knowledge of the Swahili language, not all who know or use Swahili are considered or consider themselves Swahili...The range of people included in the term Swahili is very great and is variable according to the context." For example, although there are many varieties of Swahili spoken in Mombasa (i.e., Mombasa Swahili community), speakers of Kimvita often claim to be the "real" Swahilis. In the process, fluid notions of insider "us" and outsider “them" Swahilis arise (Wald 1985: 133). Wald (ibid: 133) elaborates that inside Swahili refers to Kimvita while outside Swahili refers to "the varieties 
of Swahili spoken by people for whom Swahili is not their first language including Standard Swahili. These Swahili varieties vary according to area and education.” In terms of geographical area, speakers of Kimvita usually identify non-coastal varieties of Swahili according to the accent and first language of the speaker. In this context, speakers of Kimvita refer to these noncoastal varieties of Swahili collectively as Kiswahili cha Bara 'upcountry Swahili', while referring to its speakers pejoratively as watokabara 'people from upcountry', to mark them as "outsiders."

In terms of education, Mombasa people who have experienced formal schooling acknowledge the importance of Standard Swahili as a tool for social mobility (Wald 1973, 1985). However, Wald (1985: 141) reports that:

While use of Kimvita as a vernacular may come under attack in the school system using standard reference points, Kimvita is inviolable. It is always the 'good' traditional speech of Mvita (ancient Mombasa). In this way, Mombasa Swahili speakers' perceptions of what is included in Kimvita may change, but Kimvita as a concept and symbol of local pride cannot be negatively appraised.

Wald elaborates that by regarding their language highly, speakers of Kimvita are using it to distinguish themselves from the other members of the Mombasa Swahili speaking community on the one hand and also to delimit themselves as a distinct ethnic group on the other. By doing so, speakers of Kimvita behave like most other East Africans who consider their ethnic language to be an inalienable part of their ethnic identity and distinctiveness. However, Eastman (1971) argues that the meaning of the term Waswahili is spatiotemporal and its definition cannot be done on linguistic basis alone. Instead, it should include other social factors such as religion, line of descent, place of 
birth and speakers' attitudes around which Swahili speakers negotiate the "us" versus "them" distinctions.

My study confirms Eastman's (1971) assertion in many ways. For example, in the course of identifying my research informants in Mombasa, both teachers and students referred me to twin brothers, Ali and Abdul, whom they identified as Waswahili. However, when I interviewed the two boys, they denied that they were Waswahili. Instead, they claimed that they were Arabs because their parents were Arabs. ${ }^{43}$ Interestingly, they reported that they did not know or speak Arabic. Instead, they spoke Swahili as their first language (Ali and Abdul 03/2011, focus group--Mombasa). This indicates that ethnic labels are sometimes used to refer to people who do not define themselves that way. At the same time, the claim by the two boys that they were Arabs but spoke Swahili as their first language contravenes the common criterion of determining one's ethnicity based on his or her mother tongue.

Since Islamic religious practice is also used in the definition of Waswahili (East man 1971), it is possible that other people at Vikapuni School regarded the two boys as Waswahili because they were Muslims. Interestingly, despite being regarded as Waswahili by others, the two boys claimed that there is no tribe called Waswahili. Instead, they reported that Waswahili is a label used when referring to people who gossip too much (Ali and Abdul 03/2011, focus group--Mombasa). Such derogatory use of the label

\footnotetext{
${ }^{43}$ The label "Arab" was used during the colonial period by many coastal people, even if they did not actually come from the Middle East. Locally, the people known as Waswahili were (and continue to be) often described as ethnically mixed, descended from an "African mother and Arab father."
} 
Waswahiliis also found in the insider-outsider distinction between the communities that perceive themselves as coastal, and those associated with the upcountry. For example, Mr. Mdepa, a teacher who resides at the coast but identifies himself as Kikuyu, told me that people from the upcountry refer to coastal communities as Waswahili meaning "lazy," or "people who always give excuses to avoid taking responsibility” (Mr. Mdepa 01/1012, interview). According to Mr. Mdepa, Waswahili do not regard education seriously. Instead, they walk around shops seeking handouts while others spend time in the beaches soliciting for money from the tourists.

The examples above add to the contested ethnicity of the Swahili people of East Africa, because the people who identify themselves as Waswahili are not defined by Swahili language only. Instead, I echo Eastman's (1984) assertion that their ethnic identity is both fluid and spatiotemporal. The perceived "ethnic neutrality" of Swahili over other languages in the choice of a national language may have been connected to this issue, but also resulted from the fact that Swahili was already widely spoken by people who would not describe themselves as Swahili.

The last factor that favored the choice of Standard Swahili over other varieties is that it was already standardized by the time Kenya gained independence in 1963. The standardization process is closely tied to the spirit of nationalism, in which a nation like any other sociopolitical unit seeks to minimize internal differences while maximizing external ones (Haugen 1972). For instance, Puttenham (1859, in Haugen 1972: 101) claims that, a "form of speech becomes a language only when it is fully fashioned to the common 
understanding, and accepted by consent of a whole country and nation.” By use of this common language, Haugen argues that the state superimposes a national form of identity, which calls upon the people to extend their loyalties beyond their personal and ethnic communities for the love of their "great" nation. The nationalistic ideal at the same time discourages the people from being loyal to any other nation besides their own.

Further, Haugen (1972: 104) argues that "since the encouragement of such loyalty requires free and rather intense communication within the nation, the national ideal demands that there be a single linguistic code by means of which this communication can take place." The national language therefore offers a sense of belonging to a nation, which eventually generates prestige. The desire for this prestige further encourages the people of a nation to learn and embrace their national language. However, Haugen's nationalistic ideal does not fit well in the case of Standard Swahili for various reasons discussed further below.

In summary, the foregoing discussion has demonstrated that there are several factors that led to the choice of Standard Swahili as the national language of Kenya. These include: its perceived ethnic neutrality; it was already standardized by the time Kenya gained independence; speakers of Swahili as a mother tongue were not economically and politically powerful; the contested ethnicity of the Swahili people; and the fact that Kenya is politically and economically dominated by a few major ethnic communities such as Kikuyu, Luo and Kalenjin. The government therefore feared that elevation of one of the "ethic languages" to the status of a national language 
might lead to ethnic antagonism. In addition, the tension between the upcountry and coastal varieties of Swahili enhanced the acceptance of Standard Swahili in Kenya. Further, the choice of Standard Swahili was favored by the fact that it is Nairobi which is the economic and political locus of Kenya rather than Mombasa, where there are highly regarded local varieties of Swahili.

Despite all these factors favoring the choice of Standard Swahili, it is important to reiterate that speakers of Kimvita regard their language highly; hence they have always considered their language a better fit as Kenya's national language. But how do the two varieties of Swahili differ from each other and why do these Kenyans think that a Kimvita-based Standard Swahili would be better than the current standard? I engage the two questions in the following section. Later in the chapter, I will compare the status of Standard Swahili as a national language in Kenya, and Tanzania-- the only other country in the world where Standard Swahili has the status of a national and official language.

\subsubsection{A comparison of Standard Swahili and Kimvita}

From a linguistic point of view, Standard Swahili and Kimvita differ in a number of ways: For example, palatal affricates in Standard Swahili correspond to dental stops in Kimvita (Bakari 1985: 159). The examples in Table (1) below illustrate that difference:

\begin{tabular}{|r|l|l|l|}
\hline & Standard Swahili & Kimvita & Gloss \\
\hline 1. & Mchanga & Mtanga & Sand \\
\hline 2. & Nchi & Nti & Country \\
\hline 3. & Kucheka & Kuteka & To laugh \\
\hline
\end{tabular}


Based on the above linguistic difference, speakers of Kimvita have a common selfstereotype, which they use to distinguish themselves from speakers of the standard, and other varieties of Swahili spoken in Mombasa (Wald (1985). That is, for the imperative form of the word 'come' (infinitive kuja), "Mvita speakers report that they use the pronunciation nDoo 'come!', while other Swahili speakers including of the standard say njoo" (p. 138). In addition, Wald (1985: 132) reports that speakers of Kimvita commonly exemplify it "by means of lexical items (words) which are poetic, archaic, or otherwise not in common use in either the standard or in other Swahili varieties." Table (2) below shows some of these words and their equivalents in Standard Swahili:

Table (2): A comparison between Kimvita and Standard Swahili.

\begin{tabular}{|l|l|l|l|}
\hline & Kimvita & Standard Swahili & Gloss \\
\hline 1. & Kenda & Tisa & Nine \\
\hline 2. & Matembezi & Miguu & Legs \\
\hline
\end{tabular}

Standard Swahili and Kimvita also differ slightly in tense marking. For instance, Wald (1973) reports that, while speakers of the two Swahili varieties acknowledge use of $a$ - and $n a$ - to mark present tense in their speech, some speakers of Kimvita attach different social values to the two tense markers. That is, "they claim that $n a$ - is a marker of Kiswahili cha nde 'outside Swahili', while $a$ - is a marker of Kiswahili cha ndani 'inside Swahili'” (p. 230). Another difference in tense marking is that Kimvita employs the tense marker $n$ - which is not found in Standard Swahili. According to Wald (1973: 231), $n$ - tense is an "overt norm of Mombasa Swahili speech community and its use is admitted to and evident in the speech of all members of the community." Some teachers in Mombasa claimed that the Standard Swahili 
spoken by students in Mombasa was negatively affected by the local varieties (I will return to this point in the next section).

Such linguistic differences between Standard Swahili and Kimvita influence speakers' perceptions toward the two language varieties, and sometimes affect Mombasa students' performance in Swahili national exams. However, some Kenyans when contesting the status and future of Standard Swahili argue that these differences are too subtle to warrant any debate. Instead, they argue that what is needed to popularize Standard Swahili is institutional and government support (Mbaabu 1985). On the contrary, some Kenyans argue that Standard Swahili is an artificial language, a fabrication of the Europeans, hence there is need to re-standardize Swahili based on Kimvita, if the notion of national language is to have its intended meaning to Kenyans (Mkude 1984; Wald 1985). Next, I focus on this rivalry between Kimvita and Standard Swahili.

\subsubsection{The rivalry between Kimvita and Standard Swahili}

Standard Swahili, as noted earlier, is based on a foreign dialect. Also, it did not have native speakers in Kenya when it was adopted as the national language. According to Eastman (1984: 309), many Kenyans contend that "what is being implemented as a national language in Kenya is, in fact, not Kenyan.” Speakers of Kimvita, for example, fear that if they embraced Standard Swahili, they would be dominated culturally. This is because Kimvita is a multifaceted symbol of their ethnic pride, unity, local identity and a carrier of their rich cultural, literary and religious history as reflected, for example, by the work of the poet Muyaka (Abdul-Aziz 1979; Mbaabu 1985; 
Wald 1973, 1985). According to Abdul-Aziz, Muyaka was a renowned nationalist poet who lived in Mombasa in the $18^{\text {th }}$ and $19^{\text {th }}$ centuries, and is regarded as a culture hero by most speakers of coastal Swahili. He wrote a massive amount of poetry and single-verse pieces on almost all subjects within the cultural and political experience of his community. Many of Muyaka's statements have become proverbial among the Swahili and his work is certainly one of the most important single sources for the study of the linguistic and literary development of the Swahili language.

Kimvita is also significant to its speakers from a religious point of view. Most of its early texts, especially Muyaka's poetry, were written in Arabic script which was introduced through Islam. Both Swahili and Islam contributed to the spread of each other in East Africa. For instance, AbdulAziz (1979: 11) observes that:

The availability of the Arabic script made it possible to record a great deal of Swahili literary and historical material, and therefore preserve it for posterity. It also directly influenced the growth of a strong literate tradition among the inhabitants of the coastal settlements.

However, Eastman (1984) reports that the association of Kimvita with the Arabs and also Islamic religion contributed to its rejection by the German and British colonial administrators in East Africa during the standardization process. They instead favored the Kiunguja variety whose literature for use in education and administrative purposes had been translated and written in the Roman script. Thus, the politics of standardization have also contributed to the antagonistic relationship between Kimvita and Standard Swahili because speakers of Kimvita felt short-changed when their variety was not picked for standardization. Eastman (1984: 305) observes that "Kimvita from then on 
seemed to offer little of relevance to the rest of Kenya, which tended to look toward the Swahili of Tanganyika."

According to Mbaabu (1985), the British colonial administrators preferred Kiunguja because in its role as a trade lingua franca, it was more widespread geographically (also see Snoxall 1984). Both Mbaabu and Snoxall report that the British colonial administers found Kiunguja more economical, and politically favorable for use in colonial administration, and the works of Christian missionaries than Kimvita. However, this decision was not well received among the speakers of coastal varieties of Swahili in Kenya especially the older generations. Since then, these older speakers of coastal Swahili have been engaged in a debate contesting the role and the future of Standard Swahili as Kenya's national language. One of their arguments is rooted in the contested origins of the Swahili language, which alludes to the rivalry between Kenya and Tanzania. To date, at least three theories have been advanced (Chiraghdin and Mnyampala 1977; Horton and Middleton 2000).

The first theory claims that Swahili language is a mixture of Arabic and coastal Bantu languages spoken by the Miji-Kenda community. The second theory describes Swahili as a Bantu language that came to Kenya following the migration of the Bantu communities from Congo. The last theory claims that Swahili originated along the Kenyan coast and spread outwards to the north as far as Mogadishu in Somalia and to the south as far as Mozambique. From the point of view of language ideology, it does not matter which of the three theories is correct. (In fact, they are not necessarily mutually incompatible as they could refer to different historical periods.) 
Instead, what matters is what people believe to be correct, because such belief influences their social acceptance of the language in question. Many of the Kenyans involved in the Swahili debate support the last theory of origin and find it pitiful that even though Swahili originated in Kenya, it is Tanzania that has more pride in using and developing the language.

The rivalry between Standard Swahili and Kimvita is also found in educational contexts where the standard is taught both for examination and communication purposes. Despite the fact that many residents of Mombasa are speakers of Swahili as a first language, there have been numerous media reports that Mombasa students' performance in Swahili national exams and job applicants' performance in written Swahili interviews have been poor. For example, Wald (1985: 51) reports how a speaker of Mombasa Swahili, "who was formerly a teacher failed to get a position teaching Swahili in a government school, because his speech was considered 'old-fashioned."' These attitudes could have been informed by the fact that Standard Swahili is perceived as "modern" in some contexts (Eastman 1984).

As mentioned earlier, my research findings indicate that teachers of Swahili in Mombasa perceive students' use of local varieties of Swahili negatively. For example, Mr. Mzuri told me that "spoken Swahili of the students in Mombasa is affected by the original dialects of Swahili. Like there are people who will talk Kimvita; they say ankuja when they want to say amekuja. Also, coastal people speak very fast because they are used to Kiswahili. So, you can end up not even hearing what they are saying in Kiswahili” (Mr. Mzuri 03/2012, interview). 
As mentioned earlier, the nationalistic ideal envisaged by Haugen (1972) does not fit well in the case of Standard Swahili in Kenya. For example, one of my students in a focus group in Mombasa, Mlosi, reported that very few students in his school spoke Standard Swahili among peers. If one spoke Standard Swahili, s/he would be perceived as somebody from afar, i.e., an outsider, because students mostly speak what they call Kiswahili cha Mtaani (street Swahili) (Form Two focus group 2, 03/2012--Mombasa). Also, some scholars such as the late Kitsao of the University of Nairobi have been skeptical about the progress that Standard Swahili has made as Kenya's national language. Kitsao (1977) charges that neither the elite nor ordinary Kenyans have embraced the use of Swahili. He says:

Swahili as Kenya's national language is not making the expected progress due to a combination of factors including two important ones. First, the layman at the 'bottom' and the fruits-of-Uhuru [independence]-eating boss in the office do not know, nor do they care to learn the language since 'there really is not the need' to do so. Second, the elite consider themselves learned in any other language apart from Swahili or, worse, their mother tongue. Clearly, therefore, if we are to realize the full benefits of having Swahili as a tool of national communication a lot still remains to be done...a pathetic case because, although Kiswahili started in Kenya, efforts to develop it have been left to Tanzania alone. (Kitsao 1977, cited in Mbaabu 1985: 188)

But why are Kenyans in general not enthusiastic about the use of Standard Swahili as a national language? There are many challenges facing Standard Swahili in Kenya. However, the main ones are stiff competition from English, especially among the educated adults. The other one is the emergent urban youth vernaculars such as Sheng, mainly associated with upcountry Kenya, and lugha ya mtaani ${ }^{44}$ associated with Mombasa. I start with the dominance of English in Kenya.

\footnotetext{
${ }^{44}$ Informants in my study used the terms lugha ya mtaa and lugha ya mtaani interchangeably. The $-n i$ in lugha ya mtaani is a Swahili locative marker.
} 


\subsubsection{English versus Standard Swahili in Kenya}

The English dominance over Standard Swahili and ethnic languages, especially in the domains associated with prestige and power is historical. According to Ngugi wa Thiong'o (1986), English and its associated attitudes is a colonial legacy that was entrenched during the last decade before independence. During this time, Ngugi Wa Thiong'o (1986: 11) claims that "English was the language, and all the others had to bow before it in deference." This attitude was carried over to postcolonial Kenya where the educated elite clung to English, hence maintaining its elitist and exclusivist status. As a result, despite the promotion of Standard Swahili as Kenya's national language in 1970, and its introduction as an exam subject in primary schools in 1985, the government has been reluctant to use it as a medium for classroom instruction in other subjects, citing practical challenges (Chimerah 1998).

Similarly, though Standard Swahili is the national language and was recently elevated to the status of an official language (National Constitution 2010), it is still common for national functions such as presidential debates to be conducted in English despite the fact that they are aimed at the general populace, the majority of whom do not understand English. Such linguistic mismatch further undermines the status of Standard Swahili as the national language. This paradoxical attitude towards Standard Swahili as a national language is not peculiar to Kenya but it is a common phenomenon in many countries in post-colonial Africa. According to Makalela (2005) and Adegbija (1994), African languages including the standardized ones face unfavorable attitudes which were entrenched during the colonial period. For example, apart from Tanzania, whose case will be discussed later in the chapter, people in many countries in sub-Saharan Africa such as Rwanda, Burundi and Botswana look down 
upon their national languages, while using former colonial languages in official functions such as education and also as tools for social mobility. In this context, Makalela (2005: 15) argues that the ruling class justifies the exclusion of African languages from domains of power using neocolonial myths such as the following:

Many of these languages are not developed, so they cannot be used in education. The costs of developing African languages are very high. Their exclusive use will block the window on the world and result in exclusion from participation in the international community. Some speakers of indigenous languages do not wish to see their languages used in education because they have a total lack of confidence in the language of these domains. European languages are neutral and have a potential for creating national cohesiveness among speakers of competing languages.

However, Makalela (2005) argues that all these claims are false. He finds it unfortunate that most governments in Africa have embraced them and crafted language policies that socially construct local languages as inferior while projecting foreign languages as superior. Also, governments in many countries in Africa have failed to develop local languages, ${ }^{45}$ arguing that their promotion would amount to tribalism, which would undermine the efforts put in place to enhance national unity. This fear further undervalues these local languages in favor of the former colonial languages. Consequently, the majority of Africans devalue local languages including those that have been elevated to national or official status. In Kenya, for example, even though Standard Swahili is the national language and an official language along with English, many schools embrace a language policy that favors English over Standard Swahili. The Mombasa school in my study required students to speak English six days a week and Standard Swahili on Friday only. One of the senior teachers, Mr. Naibu, told me that it was a way of controlling noise and elaborated this approach as follows:

\footnotetext{
${ }^{45}$ By developing languages, Makalela means standardizing and codifying them to be used in conducting official functions such as educational media of instruction.
} 
To the school, when you are speaking mother tongue or Kiswahili, we feel that you are breaking the school rules. It is also a way of controlling noise because most of them can't speak English. So if we allow them to speak mother tongue, they will make a lot of noise because they know the words; they can koroga them. ${ }^{46}$ They can put in any words. But in English, they can only use stable words. So, it is for their own benefit and also a way of raising a cultured society. A cultured society where we are sticking to the norms of civilization because mother tongue and Kiswahili will always promote some violence and use of abusive words. (Mr. Naibu 01/2012, interview)

Some students in this school, especially senior prefects, have bought into the above

language ideology that speaking Swahili is retrogressive, while speaking English is

progressive. For example, in one of the focus groups, a student, Oleceleb, criticized

the use of Swahili harshly in favor of English saying that:

Kiswahili inaonyesha mtu mwenye ako old-fashioned; you are not into change. Kwa sababu the whole world right now tunaenda, as in universe imekuwa kitu moja. And everyone is talking in English. Saa wee peke yako ndiye unabaki ukiongea Kiswahili. Saa inaonekana aah, wee uko na ushamba mwingi ama kitu kaa hio. English on the other hand inakupatia an upper hand. You feel like.. English kitu kwanza itakuonyesha kaa this is a guy who knows what he wants in life, huyu ni mtu focused, a person with a future. Unaona? Kwa sababu English is an official language, when you are talking in English itaonyesha this is someone who knows what he is doing yaani. (Form Four focus group 2, 02/2012--Mombasa)

Kiswahili shows that the speaker is old-fashioned you are not into change. Because the whole world right now, we are going. As in, the universe has become one thing and everyone is talking in English. So, you would be the only one left speaking Kiswahili. So, you appear to be so 'country', something like that. English on the other hand gives you an upper hand. You feel like, English is something that first shows that this is a guy who knows what he wants in life, this is a focused person, a person with a future. You see? Because English is an official language, when you are talking in English it shows that you are someone who knows what he is doing. (Form Four focus group 2, 02/2012--Mombasa)

This language policy of the Mombasa school was revised in the course of the semester to one that required students to speak English at all times. Subsequently, notices reinforcing this change were posted in all the classrooms and on the main notice board (see Appendix E). ${ }^{47}$

The unequal treatment of English and Standard Swahili was also evident in other social spaces within the school. For example, English was allocated eight

\footnotetext{
${ }^{46}$ Koroga means 'mix' in Standard Swahili, though it is used in a demeaning way in colloquial Swahili, especially when expressing displeasure towards someone's way of speaking.

${ }^{47}$ It is possible that this drastic change of policy was due to my presence in the school.
} 
lessons a week while Standard Swahili had five in the main school timetable. Also, English dominated over Standard Swahili in the notice boards located in the classrooms, teachers' lounge and outside departmental offices, where internal memos and official announcements were posted. Even though the Nakuru School did not have such a strict language policy, the majority of the teachers that I interviewed also favored English while disparaging Standard Swahili. English also dominated Standard Swahili in the print materials and announcements posted in the various notice boards within the school. English dominated in that very few printed materials and announcements were written in Standard Swahili. The only other language variety that I observed in these notice boards was Sheng, especially in the guidance and counseling and co-curricular departments such as clubs and sports.

In both schools, the unequal treatment between English and Standard Swahili was also reflected in the newspaper readership. The administration ordered for several copies of the English-language newspapers, The Standard and Daily Nation, from Monday to Friday, but only one copy of the Swahili language paper, Taifa Leo. These newspapers were meant for the whole school though people were expected to read them in turns because the copies were few. However, the English copies in both schools were distributed among teachers while the Swahili one was left at the main gate, where it was picked up by one of the nonteaching or surbodinate staff who then passes it over to others. These are the people who did manual jobs in the school such as trimming hedges. Initially, I thought that the Swahili newspaper was given to them because of their love for Swahili. However, I later discovered that teachers associated Swahili with people with low levels of education. Even teachers of Swahili always sought to read the English newspapers unless there were sample Swahili national 
examination papers included in the Taifa Leo. Mr. Naibu, the teacher mentioned earlier from Mombasa, commented that the only other time one could find a teacher looking for Taifa Leo was when the newspaper had coupons or raffles (Mr. Naibu 01/2012, interview).

I also sought to know the newspaper readership outside the school such as in the streets, to establish the attitudes people in the wider society had towards English and Standard Swahili. According to Mr. Naibu:

People usually go for the Daily Nation. Taifa Leo is popular in coast because people like Kiswahili especially those old wazees. ${ }^{48}$ But on average, people who are learned, you will not see many go for Taifa Leo. What I know is that the few people who will go for Taifa Leo are those wazees or those who are semi-illiterate. (Mr. Naibu $01 / 2012$, interview)

The association of Taifa Leo (the only Swahili national newspaper in Kenya) with old people might have negatively affected young Kenyans' perceptions toward Standard Swahili.

From another point of view, teachers in both Nakuru and Mombasa claimed that careers associated with Standard Swahili were few and most of them were limited to East Africa, the region where Swahili is spoken. One teacher in Nakuru, Mrs.

Kizito, argued that it was important for students to excel in English, because it was an international language and would therefore make them more competitive in the job market after graduation (Mrs. Kizito 11/2011, interview).

Closely related to the claim above - that graduates should excel in English exams and embrace it to be competitive in the job market - is the fact that many adults in Kenya associate English, but not Standard Swahili with being educated. For example, I asked Mr. Mgema, a teacher from Nakuru, to comment on why use of

\footnotetext{
${ }^{48}$ The '-s' at the end of Wazee is the English plural marker that has been suffixed on to the Swahili stem. Though Wazee in Standard Swahili means old men and women, in my own observation, women rarely read Taifa Leo.
} 
English outside school settings seemed more acceptable than Standard Swahili. This is despite the fact that Standard Swahili is the national language and the official language along with English. He responded from a historical point of view that:

English was originally for the learned. So if you have gone to school, you should be able to speak English. If you go out there and try to speak very good Kiswahili, it doesn't communicate you going to school at all. Also, the better English you can speak the more official you are. (Mr. Mgema 11/ 2011, interview)

These kinds of attitudes, especially when expressed by figures of authority, significantly undermine Standard Swahili as a national and official language in Kenya. This is because the same teachers are usually involved in students' career guidance. It is therefore unrealistic to expect such teachers who have a low regard for Standard Swahili to encourage students to embrace it. Instead, such teachers often encourage students to pursue English or science oriented careers, arguing that they would lead to more prestigious and well-paying jobs.

Some speakers also expressed attitudes that indicate that their newspaper readership is influenced by social class aspirations. For instance, Mr. Naibu, the teacher from Mombasa, reported that he could not imagine himself reading Taifa Leo in public spaces such as when riding in a matatu, because people might think that he was not serious or that he was a retired primary school teacher. ${ }^{49}$ He elaborated that high school teachers did not read Taifa Leo unless one was a teacher of Swahili, because reading Taifa Leo would mark the reader as semi-illiterate. Further, Mr. Naibu claimed that many people avoided reading Taifa Leo because it was cheap, costing 25 Kenya shillings only. As such, Mr. Naibu argues that if people saw you buying Taifa Leo, they would think that it was all you could afford (Mr. Naibu 01/2012, interview). I recorded similar sentiments from some teachers and students in

\footnotetext{
${ }^{49} \mathrm{Mr}$. Naibu's use of the expression "not being serious" here suggests that one would be demeaning himself or herself or s/he is associating with a lower social class.
} 
Nakuru, suggesting that speakers' language choices in Kenya, whether during verbal interactions or in newspaper readership, are influenced by social factors such as education, career ranking and class aspirations. All these factors privilege English over Standard Swahili, especially because use of Standard Swahili has limited social rewards outside classroom settings, and associating with it sometimes puts one's identity at stake.

Overall, I argue that the unequal perception between Standard Swahili and English in educational or official settings, i.e., associating English with being educated, formality/authority and prestige, while associating Swahili in general with illiteracy, informality and casualness, has led many Kenyans to perceive speakers of Swahili and speakers of English unequally. As a result, many Kenyans, especially adults, are usually more mindful about speaking "correct" English than Standard Swahili. In this regard, even though the government policy appears to expect teachers and students to promote and embrace the two officially sanctioned languages equally, they do not do so in actual practice. This is in line with my research findings that very few teachers and students spoke Standard Swahili outside the classroom, especially if the situation would allow for other language choices. In both Nakuru and Mombasa, many teachers and students claimed that they avoid speaking Standard Swahili because it is "hard." I examine this claim in the following section starting with teachers. (I will discuss students' attitudes on the antagonistic relationship between Sheng and Standard Swahili later in the chapter.)

So, what do teachers mean when they claim that Standard Swahili is "hard?" Some teachers claimed that the curriculum of Standard Swahili had changed a lot since the time they were students; it was almost as if current students were learning a 
different language altogether. For example, Mrs. Tajiriba, the head of the languages department in Nakuru, expressed her frustration with the kind of Standard Swahili being used by some TV stations nowadays. She argued that some Swahili newscasters and reporters employ vocabulary such as Paruwanja la Mihadarati, 'Drug Syndicate', that ordinary citizens cannot understand (Mrs. Tajiriba 11/2011, interview).

Another teacher of English and Swahili in Nakuru, Mr. Gutz, reported that people feel that when they adhere to the standards, pronunciation of words becomes tedious and a "mouthful." 50 He then summarized that what makes Standard Swahili difficult for use in peoples' daily interactions is the many and complex grammatical rules, lack of enough Swahili vocabulary and lack of familiarity with Standard Swahili because they are not used to speaking it. As such, Mr. Gutz claimed use of Standard Swahili outside the school is left to very few people, mostly academicians (Mr. Gutz 10/2011, interview). Interestingly, even though there are media reporters who employ English vocabulary that many Kenyans do not understand, e.g., the term "promulgation," which was used when launching the new Constitution, both Mrs. Tajiriba and Mr. Gutz did not criticize them. This suggests that the difficulty of vocabulary is not really the main issue but rather the value attached to each of the two languages. The high value attached to English encourages speakers to embrace it while turning away from Standard Swahili.

I recorded similar sentiments from several other teachers in Nakuru and Mombasa who said that the Swahili employed in the Taifa Leo newspaper is "hard" to use in ordinary conversations. One of them, Mr. Mgema, from Nakuru, reported that

\footnotetext{
${ }^{50}$ I didn't get the exact meaning for 'mouthful' but he later insinuated that one of its meanings is being wordy and monotonous.
} 
people in actual practice, e.g., in the marketplace do not use Standard Swahili because many of them do not understand it. Instead, they use:

What I would call street Kiswahili. What I mean by street Kiswahili is Kiswahili for communication; Kiswahili that is spoken that is not standard. That is what I mean...So, if I want, eh, I can say patia yeye maji ['pass him water'], instead of saying mpe maji ['give him water']. You see I am not following the strict rules of Kiswahili. But otherwise it's Kiswahili. It's the Kiswahili that I would meet with anybody and talk. That is what I mean by street Swahili. (Mr. Mgema 11/ 2011, interview)

I sought to know why Mr. Mgema claimed that people did not understand Standard Swahili while it was being taught in schools to be embraced for communication purposes, especially in cosmopolitan areas. As if trying to prove his claim, $\mathrm{Mr}$. Mgema narrated how he organized a seminar and prepared a registration form in Standard Swahili, which had the following column titles: Jina 'name', Anwani 'address', and Makazi 'residence'. He then distributed the forms to the seminar attendees for them to fill out and return them when they were done. To Mr. Mgema's surprise, one lady walked up to him and asked, “Na sisi ambao hatuna kazi?”, 'What about us who are unemployed?' According to Mr. Mgema, this lady thought that the column title Makazi meant the plural of Kazi 'work/job' in Standard Swahili, while it actually meant place of residence. Mr. Mgema then wondered rhetorically how the government could expect the general population to communicate in Standard Swahili in their daily interactions if educated people could not understand such basic Swahili. Another group of teachers in Nakuru said that it is teachers of Standard Swahili who contribute to its unpopularity because they do not use the same kind of Swahili during Swahili lessons, and when interacting with people outside the classroom. For example, I had a casual conversation with Mr. Migingo, a teacher of geography, about the languages spoken in his school. He claimed that he found Swahili language attractive and therefore enjoyed socializing with Swahili teachers 
outside the classroom. However, he said that he did not like the way teachers of Standard Swahili drastically changed their tone, posture, and even vocabulary when teaching Swahili because the switch made them sound too formal, serious and unfriendly (Mr. Migingo 10/2011, casual conversation). Later on, we were joined by Mrs. Kantai, a teacher of Swahili who was heading for a lesson in one of the grade 10 classes, but decided to spend some time with us as she waited for the bell. A few minutes later, the bell rang and Mrs. Kantai left for her Swahili lesson. As time went by, Mr. Migingo and I could hear her voice addressing students in Standard Swahili. Mr. Migingo then gestured to me pointing towards Mrs. Kantai's class and asked sarcastically, "Is that the same Kantai we were laughing with? These are the people making our students dislike Swahili. Why has she become so formal and serious?" (Mr. Migingo 10/ 2011, casual conversation). According to Mr. Migingo, a teacher should be friendly and accessible both within and outside the classroom.

Another teacher, Mr. Mwungwana, expressed similar sentiments. He said that, "even though teachers will try speaking in Standard Swahili, they often 'slide' or revert to other languages or mix them in their conversations. Consequently, a teacher who speaks in Standard Swahili throughout is perceived as anti-social” (Mr. Mwungwana 10/ 2011, casual conversation). As if to justify his claim, Mr. Mwungwana asked me whether I had had a chance to interact with a particular female teacher of Swahili that he personally found antisocial. He reported that this teacher always spoke Standard Swahili even when responding to teachers who spoke to her in English or other languages. Mr. Mwungana suggested that I should interview her and experience it myself. From the point of view of language ideology, what is important from Mr. Mwungwana's sentiments is that Kenyans rarely use standard language in 
their social interactions outside formal situations. Instead, language switching and mixing is usually the norm (Myers-Scotton 1993). However, it is still worth probing further why the teachers I have discussed so far seem to single out Standard Swahili rather than English. Further scrutiny will help to reveal whether there is a difference between teachers' and students' attitudes toward Standard Swahili on the one hand and English on the other.

To some extent, Mr. Migingo's and Mwungwana’s sentiments are accurate. I sat and observed several Swahili lessons of one particular male teacher in Nakuru, Mr.Wamalwa, and noted that students rarely spoke. Even when they responded to Mr.Wamalwa's questions, they gave one word answers or the "No-Yes" or "mhh!" responses. Outside the classroom, I noticed that Mr. Wamalwa spent much of his time in the Swahili office. Whenever he came to the teachers' lounge during tea and lunch breaks, he would greet and briefly chat with his colleagues in Standard Swahili, then withdraw and take his tea quietly. Interestingly, there was another male teacher of Islamic studies, Mr.Mpoa, originally from the coast but who at the time resided in Nakuru. He spoke Swahili most of the time when interacting with teachers and students, but I never heard anybody criticizing him the same way other teachers of Swahili discussed above were criticized. Later on, I learned from the student informants that Mr. Mpoa was their favorite teacher for various reasons that include: 1) they enjoyed listening to his Swahili; 2) he sometimes spoke to them in Sheng; 3) he understood their problems; and 4) he had a unique dressing style--he consistently put on high-waist pants with suspenders and well ironed shirts. Some students claimed that Mr. Mpoa had swag (Hora 09/2011, casual conversation). Next, I 
examine why teachers and students in Nakuru did not criticize Mr.Mpoa for speaking Swahili as they did other teachers.

Just like teachers, some students in Nakuru reported that they enjoyed listening to some variety of Swahili. However, I later found that it was not Standard Swahili but varieties spoken at the Kenyan coast. For instance, recall from Chapter One about a student in Nakuru, Kadonye, who asked me, "Mbona Kiswahili ni kitamu lakini kigumu?", 'Why is Kiswahili sweet but difficult?' (Kadonye 09/2011, casual conversation). I decided to find out what Kadonye meant by engaging him in a discussion on what students in his school said about Swahili in general. I will limit my discussion here to the first part of the claim that Swahili is "sweet." The other part that Swahili is "hard" will be discussed in the section focusing on the antagonistic relationship between Sheng and Standard Swahili because it reveals the language ideology that motivate many young Kenyans to turn away from Standard Swahili in favor of nonstandard language varieties such as Sheng and lugha ya mtaa.

So, what variety of Swahili is "sweet" and why? The student Kadonye told me that he enjoyed listening to Swahili radio commentators of soccer in Kenya and conversations between people from the coast. Coincidentally, I personally developed a love for Swahili language from listening to radio soccer commentaries in the ' $80 \mathrm{~s}$ and ' 90 s, when as a boy I would bring a small radio to listen to, while tending the family cows in the forest. I used to admire their fluency, accent and word choice. Even though the main radio stations in Kenya have had soccer commentators from both coast and upcountry, the ones with the largest number of fans are from the coast or were educated at the coast. These included Ali Salim Manga, Mohammad Juma Njuguna and Mambo Mbotela. The claim that Swahili is "sweet" was not limited to 
Nakuru. I recorded similar sentiments in Mombasa among students from upcountry

Kenya and also among coastal students who had the privilege of visiting upcountry.

For example, the transcript below is from a female student called Zawadi, in a mixedgender focus group, who had visited her relatives in Nairobi and noted that the people she met on different occasions admired her spoken Swahili. She said:

Kama nilivyoenda Nairobi, nilienda kwanza, nilikuwa nimeenda kununua pojo. Sasa nikakuta kule wanaiita ndengu. Halafu vile nilikuwa naongea sasa, kila nilipokuwa naenda dukani, hao watu wa dukani walikuwa wanataka niongee, yaani huyo cousin yangu asiongee kwa sababu wanapenda kile Kiswahili ambacho naongea nacho. Pahali popote ambapo tutakaa watoto wanataka tu mimi niongee kwa sababu wanapenda hicho Kiswahili ambacho nilikuwa naongea. (Form Two focus group 2, 03/2012--Mombasa)

When I went to Nairobi, I went out with my cousin to buy pojo ['green grams']. I found that people in Nairobi call them Kunde. Then, I realized that people there admired my Swahili and whenever I went to the store with my cousin, I realized that people always wanted me to talk because they liked my Swahili. Everywhere we went; kids wanted me to talk because they loved the Swahili that I was speaking. (Form Two focus group 2, 03/2012--Mombasa)

From the way Zawadi constructed herself socially as a speaker whose language amazed everyone she met in Nairobi, I asked her to describe the Swahili that she spoke there and why she thought Nairobi people admired it. She responded that:

Saa unajua nilipofika kule, nilikuwa naongea Kiswahili mufti kwa sababu nilikuwa nawaambia kuwa nimetoka Bongo [mmh, Tanzania?MW], eeh. Halafu pia walikuwa wanaamini. Halafu mtu akishaniambia kitu kidogo nilikuwa nishampaka yaani nampa lake. Namnyamazisha. (Form Two focus group 2, 03/2012--Mombasa)

You know when I arrived in Nairobi, I was speaking pure Swahili. I used to tell them that I come from Bongo [Tanzania? MW] eeh, and they would believe me. So, whenever someone told me something, I would respond in a way that left him/her with no words. That is, I would silence him/her. (Form Two focus group 2, 03/2012-Mombasa)

Zawadi's guise that she spoke such amazing Swahili because she is from Tanzania points to an ongoing debate pitting Tanzanians versus Kenyans mentioned earlier, about the origins of Swahili language and who between them speaks better Swahili. In this regard, when some Kenyans from the upcountry and Tanzanians meet, it is 
common to find them teasing one another using jokes such as, "Kiswahili was born in Zanzibar, grew up in Tanganyika, died in Kenya and was buried in Uganda and its ghost flew to Congo" (Africa Review, April 26 ${ }^{\text {th }} 2013$ ). Another version of this joke is that, "Kiswahili was born in Zanzibar, grew up in Tanzania, was adulterated/fell sick in Kenya, died in Uganda and was buried in Congo" (The East African Magazine, January $\left.17^{\text {th }} 2011\right)$.

While such jokes would not play well among the older generations of Swahili speakers at the Kenyan coast, some Kenyans from the upcountry enjoy such teasing and often buy into the ideology that Tanzanians speak better Swahili. However, what is important in this context is not whether the Swahili spoken in Tanzania is better than the one spoken in Kenya. Instead, it is the ability of young Kenyans to associate and dissociate with the various language ideology to generate social rewards. In this regard, the attitudes that they express towards the various language varieties in their repertoire are contextual and sometimes contradictory. For example, I asked Zawadi, the student from Mombasa, why she falsely told people in Nairobi that she was from Tanzania and she responded that:

Yaani, wanachukulia Wabongo juu. Wakenya wanapenda Kiswahili chao yaani vyenye wanavyoongea. Hata hapa tuna wabongo wengi skuli. Wanapendwa sana vile wanavyoongea. Yaani watu wanataka wajumuike nao waone vile wanavyoongea. ${ }^{51}$ (Form Two focus group 2, 03/2012--Mombasa)

That is, Kenyans regard Wabongo (Tanzanians) highly and love their Swahili. That is, they love listening to the way Tanzanians speak. Like here in our school, we have many students from Tanzania. Kenyan students love the way these Tanzanian students speak and therefore like spending time with them. (Form Two focus group 2, 03/2012--Mombasa)

\footnotetext{
${ }^{51}$ The majority of students in this school were from ethnic communities usually perceived as from upcountry such as Kambas, Luos and Kikuyus.
} 
Zawadi's response reveals that she was aware of the belief that Tanzanians speak better Swahili than Kenyans, which is common in upcountry Kenya. As such, she was able to elevate herself socially over the people she interacted with in Nairobi.

However, Zawadi and other members of her focus group later claimed that they spoke lugha ya mtaa, and criticized Tanzanian youth for speaking "pure" Swahili. This observation conforms with previous research focusing on urban youth vernaculars in post-colonial Africa in that speakers of these language varieties operate within a “paradox of norms" (Kiesling and Mous 2004; McLaughlin 2009).

Based on Zawadi's claim that Kenyan students in her school enjoyed listening to the Swahili spoken by the students from Tanzania, I asked them what they believed Tanzanians say about the Swahili spoken by Kenyans. One male student called Maunga responded that:

Mimi nimegundua kuwa Kiswahili cha Kenya wanakidharau. Wanasema sisi tunawajua wale ndio wenye Kiswahili. Sasa wanasema sisi Wakenya tunamisuse Kiswahili; tunachanganya ndimi ovyo. Hizi lugha za mtaani haziko kwenye Kiswahili mufti. Saa ni kama wanatudharau sisi Wakenya wanasema sisi hatujui Kiswahili tumecopy wao. [...]

Aah, kule huwanga wanatumia lugha rasmi. Yaani hata wakiongea utasikia ni lugha safi, ni lugha mufti huwanga hawakosei. Hiyo ndio kitu iko. Ndio wanasemanga sisi Wakenya tumechanganya, tunatumia Kiswahili cha Mtaa ni.

(Form Two focus group 2, 03/2012-Mombasa).
I have discovered that Tanzanians despise the Swahili that is spoken in Kenya. They say that Kenyans acknowledge that Tanzanians speak the real Swahili. Like now they are saying that Kenyans misuse Kiswahili; we mix languages carelessly. These street languages are not found in the pure Swahili. It is like they despise us Kenyans. They say that we do not know Kiswahili [and that] we have copied from them instead. [...]

Aah, there [in Tanzania], people usually speak official language. That is, even when they speak, you will hear that their language is clean; it is a pure language, they never make mistakes. That is how the situation is. That is why they usually say that we Kenyans usually mix languages; we speak street Swahili.

(Form Two focus group 2, 03/2012--Mombasa)

Even though the various claims in Maunga's response can be contested from an empirical point of view, they are important from the point of view of language ideology. Recall that language switching and mixing is the norm in Kenya, and use of 
Standard Swahili has limited social rewards. In contrast, Maunga's response suggests that "linguistic purity" and "grammatical correctness" are highly regarded in Tanzania. Such language ideology may have contributed significantly to the success of Standard Swahili as a national and official language in Tanzania more than in Kenya. For instance, Billings (2009: 590) reports how contestants in beauty pageants in Tanzania "expressed repeatedly the importance of their speaking during the competitions in a way that is free of kuchanganya 'mixing'- that is speaking Swahili without English, or English without Swahili." According to Billings (ibid: 591), these expectations are for language use in "formal, public speech, as taught in school. However, in less formal settings, speaking Swahili without substantial English borrowings is not only difficult but also undesirable."

A similar scenario exists in the Tanzanian parliamentary 'Bunge' proceedings, which is a formal speech event requiring speakers to use Kiswahili as a symbol of Tanzanian nationalism, but speakers often engage in codeswitching between Swahili and English (Bwenge 2002). The formal-informal distinction with regard to the use of Swahili in Tanzania differs from the Kenyan case in that while Tanzanians may be reprimanded for not using "pure" Swahili in official contexts, many Kenyans including government officials get away with it easily.

In addition to the claim that Tanzanians speak "pure" Swahili, another student called Georgia from the same focus group with Zawadi and Maunga, added that Tanzanians speak more "respectful" language than Kenyans, saying: 
1

Kule wanaongea lugha safi na ya heshima kabisa. Kwa sababu kule si rahisi upate kama askari hivi akuitishe tuseme license hivi kwa nguvu hivi kama huku Kenya. Kule watu wanakwambia kwa upole. Yaani kama wanarequest yaani. Wanakuitisha tu kwa heshima.
There [in Tanzania], people speak clean/pure and very respectful language. For example, it is not common to find police in Tanzania demanding or forcefully asking you to produce a driver's license like here in Kenya. There, people tell you politely. That is, it is like they are requesting you. They ask you courteously.

(Form Two focus group 2, 03/2012--Mombasa).

Even though Maunga's and Georgia's claims that police in Tanzania always speak Standard Swahili is contestable, the contrast that they make between the Swahili varieties associated with Kenyan and Tanzanian police is important from the point of view of language ideology. The contrast makes it clear that people in authority and the identities that they project as they interact with the citizens greatly influence ordinary peoples' language attitudes. In the Kenyan case for example, police occupy a central place in peoples' social life in their role of maintaining law and order. However, since police are known for harassing and abusing people even when they have not committed any crime, it is common to find ordinary Kenyans using the nonstandard variety of Swahili that police speak when interacting with them, rather than the standard. They do so in order to be able to negotiate for freedom in case they are arrested.

The variety of Swahili associated with the police in Kenya has no particular name but it resembles the pidginized Ki-Settla Swahili variety, which was spoken by the European settlers and colonial administrators during the colonial period. According to Whiteley (1993: 65), Ki-Settla is characterized by "limited vocabulary and a highly attenuated grammatical structure. ” It developed among the settlers 
especially because of the paternalistic attitude that they had towards Africans. The settlers thought that speaking Swahili "correctly" would be demeaning themselves, and elevating Swahili to the level of English. As a result, Mkangi (1984: 336) argues that settlers "ended up debasing the language by creating the 'Ki-Settla dialect'."

From a sociolinguistic point of view, colonial administrators and settlers used Ki-Settla to create and also perpetuate social distance between themselves and Africans (Fabian 1991). Also, Ki-Settla was associated with power and authority (Mkangi 1984; Whiteley 1993). Such use of Ki-Settla affected the attitudes that Africans had toward this variety of Swahili. Since Kenya inherited colonial administrative structures, Ki-Settla or a version of it may have been embraced by post-independence police forces, because of its association with authority. Similarly, Swahili in Uganda, where it is associated with an oppressive police force, prostitutes or criminals, is generally perceived negatively (Kawoya 1984). In the case of police, Kawoya (1984: 39) reports that:

During President Amin's rule, Kiswahili moved from the barracks where it was associated with the soldiers to the public. Soldiers intermingled more frequently with the public than ever before and in the course of time, civilians picked some Kiswahili words and phrases from them. Again, roadblocks became a daily feature in the life of Ugandans; and people had in one way or another to learn some kind of Swahili in order to avoid harassment when confronted with soldiers at roadblocks. Some knowledge of Swahili became as it were, a conditio sine qua non because the use of English or any other language other than Kiswahili would be tantamount to an insult as far as those manning those roadblocks were concerned. Even at present one hears people say that they want to learn some Kiswahili to use at roadblocks or not to be completely at a loss when addressed in the same at roadblocks.

The various examples demonstrate that people in authority greatly influence peoples' attitudes toward the lenaguage in question.

In summary, the foregoing discussion has demonstrated that both teachers and students in Mombasa and Nakuru use Standard Swahili in very limited contexts. The 
main difference is that while many teachers prefer English and sometimes their ethnic languages over Standard Swahili, many students prefer Sheng and other related language varieties unless the situation strictly demands that they speak the officially sanctioned languages. The discussion also revealed that there are speakers who find Swahili "sweet," hence they like listening to it. However, the Swahili that they referred to was not the standard but coastal varieties. In this regard, some Kenyans from the coast who are aware of this language ideology often take advantage of it when they visit upcountry, as in the case of the student Zawadi when she visited Nairobi.

In addition, the discussion revealed that the way people in authority such as police employ language, significantly affects ordinary citizens' attitudes toward that language. For example, the association between Swahili and oppressive police force in Uganda negatively affected peoples' attitudes toward Swahili in general. Also, the fact that teachers in Kenya rarely speak Standard Swahili and some do not mind speaking the language "incorrectly" significantly undermine its status as a national language. For such reasons, some scholars have argued that Standard Swahili as a national and official language has been more successful in the neighboring Tanzania, which is more ethnolinguistically diverse than Kenya. As mentioned earlier, the success of Standard Swahili in Tanzania is relevant to my project because it is the only other country in the world where Standard Swahili is both an official and national language. Also, there is a common believe in East Africa that people in Tanzania speak "pure" Swahili including the youth. I discuss these reasons and others below. 


\subsubsection{Comparison between the status of Standard Swahili as a national and official language in Kenya and neighboring Tanzania}

Tanzania is a multilingual country but according to Brock-Utne (2005: 51), there are several factors that favored its choice of Standard Swahili as a national language over other local languages when it gained political independence in 1961. From a historical point of view, Brock-Utne reports that Standard Swahili was the main medium of instruction in African education and colonial administration in Tanzania. The German colonialial administrators had shifted the orthorgraphy of Swahili from the initial Arabic script to the Roman alphabet. This work of translation was motivated by the desire to steer away Tanzanians from Islamic culture, but was also informed by the colonialists' paternalistic attitude that Tanzanians could not learn to speak German sufficiently well. Therefore, the colonialists' efforts to promote the use of Swahili as a lingua franca was meant to deny Tanzanians the privilege of learning German.

However, these efforts turned out to be a blessing in disguise because they enhanced the growth, development and spread of Swahili across the country. In this regard, Tanzania at independence inherited colonial structures where Swahili was already established. So, when Julius Nyerere, the first president of Tanzania, called for the adoption of Standard Swahili as the national language in 1961, he was supported by the majority of politicians (Brock-Utne 2005). In addition to the support from fellow politicians, Nyerere also made significant personal contributions in popularizing Swahili, such as translating plays written by Shakespeare. He also addressed the Parliament and other institutional meetings in Swahili. Further, he encouraged borrowing from ethnic languages both as a way of preserving them and 
enriching Swahili. Interestingly, Tanzanians did not seethis practice as language mixing, indicating the inherent paradoxical nature of homogenous language ideology.

Such political and institutional support contributed to the embrace of Standard Swahili by the majority of Tanzanians not just as one of the languages of Tanzania but as a marker of Tanzanianness, i.e., national identity (Brock-Utne 2005; Eastman 1984). For example, Brock-Utne reports that in 2004 , Swahili was spoken by above $90 \%$ of the country's population. Currently, the population of children acquiring it as their first language is on the rise. Many more are acquiring it with their parents' language simultaneously. Standard Swahili in Tanzania has also continued to enjoy institutional support. For instance, it is the main medium of classroom instruction at the primary school level, and in some teacher training colleges. It is also the main language of communication in Parliament, the Supreme Court, lower courts, and the Kiswahili Research Institute, and there are several newspapers published in Standard Swahili.

From the point of view of national unity, Brock-Utne (2005) says that people in Tanzania are not normally asked about their ethnicity. Even where such questions are asked such as during national population censuses, citizens are at liberty not to answer. According to Brock-Utne, the question about ethnicity is usually avoided in order to combat tribalism in the country. In my own observation, this is unlike Kenya where ethnic identity is one of the key pieces of information sought during the national census. ${ }^{52}$ In educational contexts, Brock-Utne reports that there was a

\footnotetext{
${ }^{52}$ As noted earlier, national politics in Kenya especially presidential elections are driven by ethnicity. People vote along ethnic lines, hence the government is always keen to know which communities have the numbers which would translate as votes during national elections. Most recently, one of the political analysts called Mutahi Ngunyi devised a formula that he called "The Tyranny of Numbers," which he used to explain that the winner of the just concluded presidential election was determined
} 
proposal in Tanzania to introduce a tripartite language policy where learning would be introduced in children's mother tongue and Swahili, then switch to Swahili and English. However, Nyerere did not support it probably to maintain his wish to unite the country and avoid tribalism. It is largely due to this policy that Swahili is spoken by the majority of Tanzanians despite the country's high ethnolinguistic diversity. However, it is worth pointing out that though Tanzania has succeeded in using Standard Swahili, a local language, in national and official functions more than many other countries in Africa, English, the language of the former colonial power, still enjoys a higher status. For example, Billings (2009: 581-82) reports that:

In Tanzanian beauty pageants, where contestants' onstage speech is the focus of explicit and implicit critique...contestants who speak English are far more likely to win than are their Swahili-speaking counterparts... In particular, a contestant's ability to speak English onstage marks her as a member of an educated elite, a desirable quality for moving up through the pageant hierarchy to Miss Tanzania and ultimately to Miss World.

While emphasizing the contrast between Tanzania's commitment to Standard Swahili and how other countries treat African languages, Batibo (1995: 68) says, “[t]he most obvious argument to adopt Kiswahili as the language of law in Tanzania is that it has, in practice, become one." The case of Standard Swahili in Tanzania thus contrasts with Kenya in many ways. For instance, when Jomo Kenyatta called for the use of Kiswahili in Kenya's parliament in 1964, he faced stiff resistance from key government officials who preferred English, further indicating that these officials still embraced those biased colonial attitudes toward Swahili. For example, recall that

way before the actual election. This is because the winner had managed to craft a political coalition between the largest and also socioeconomically dominant ethnic groups, i.e., Kikuyus and Kalenjins. As such, Mutahi Ngunyi concluded that the president who was to be sought through the electoral process was instead determined at the closure of the voter registration process which was done three months before the general election. For more details see: http://www.youtube.com/watch?v=977VqPqcQvk 
Charles Njonjo, the Attorney General at that time was opposed to Swahili claiming that it has Arabic roots. According to Mazrui and Mazrui (1995: 77-78), Njonjo argued that "Swahili is not our language, and it is not our mother tongue: it is a foreign language as much as English is a foreign language."

Due to the fact that many Kenyan politicians have not embraced Standard Swahili, political discourse in Kenya, unlike in Tanzania, is mostly conducted in either English, ethnic languages or contact varieties of Swahili which are closer to Sheng than to Standard Swahili, or via code-switching among other nonstandard language practices (Daily Nation Newspaper, September $4^{\text {th }} 2010$; Harries 1976). Some politicians and other influential institutions such as the media, business firms and religious organizations have been appropriating Sheng strategically in an attempt to win the youth. For example, Mr. Mgema, the teacher from Nakuru introduced earlier, observed that:

The adults who speak Sheng could be in various areas. One, they could be dealing with youths. So, if they are dealing with youth, they must speak in the language they [youth] understand. I don't know if you heard eh, Rafael Tuju [a presidential aspirant in the 2013 General Elections] when he went to Kisumu, when he was telling eh, 'naelewa kugrow bila maparoo' [Sheng for 'I understand what it means to grow up without parents']. (Mr. Mgema 11/ 2011, interview)

Mr. Tuju's use of Sheng indicates that even individuals seeking to become the president of Kenya have subscribed to the language ideology that Sheng is the language for reaching the youth. Such language ideology further undermines the role of Standard Swahili as a national language, and also suggests that Standard Swahili in most cases enjoys this status only in theory. I now turn to the antagonistic relationship between Standard Swahili and Sheng. I seek to unravel what informs some speakers' claim that Sheng would be a better national language than Standard Swahili, as indicated in the quotation at the beginning of this chapter. 


\subsubsection{Standard Swahili versus urban youth vernaculars such as Sheng: theory versus practice in the national language policy}

Despite intensive efforts in schools to promote Standard Swahili as a national language, my field observations indicate that many young Kenyans, especially in cosmopolitan neighborhoods rarely speak Standard Swahili outside classroom settings. Instead, they prefer nonstandard language varieties which they described using various labels such as the following. One group of speakers used the term Kiswahili cha Kawaida 'Ordinary Swahili' (referring to Swahili for communication purposes). Another category of speakers, mainly from Mombasa, reported that they spoke lugha ya mtaani. The last category, and this constitutes the majority of young Kenyans in Nakuru and some in Mombasa, reported that they spoke Sheng. ${ }^{53}$ Even though speakers of the various nonstandard language varieties have all turned away from Standard Swahili, speakers of Sheng have gone a step further by envisioning that Sheng could be Kenya's future national language. I now focus on this disconnect between schools' efforts to promote Standard Swahili and Sheng speakers' language use in actual practice.

From the point of view of language ideology, the government's choice of Standard Swahili as Kenya's national language was informed by the notion of "homogeneism." According to Blommaert and Verschueren (1998), "homogeneism" regards linguistic diversity as a threat to national unity, and therefore seeks to homogenize internal cultural and linguistic diversity. Consequently, the national ideal conflicts with the language varieties such as Sheng that borrow linguistic material

53 These labels were discussed in Chapter One, which focuses on what speakers call Sheng. However, since Sheng is socially defined, it is possible to find one word or expression fitting in two or more of these categories. Also, as noted in the previous section, some speakers associate and dissociate with the various language varieties to maximize social rewards. 
from others and regards them as "impure" and a threat to national unity. In the Kenyan context, the national ideal is paradoxical in various ways. Even though Standard Swahili is socially constructed as a "pure" language, it has abundant loan words from English, German, Arabic and other languages. It is also possible that some Sheng vocabulary or expressions have infiltrated into Standard Swahili over time. For example, it is common to find Kenyans regarding some expressions as Standard Swahili in one context and Sheng or street Swahili in another. A good example is the expression wacha mpango wa kando 'desist from extra-marital affairs', commonly used in the media campaigns against HIV/AIDS. Even though all the words used in this expression are Standard Swahili, the expression is often interpreted by speakers to be either Standard Swahili or Sheng. Another example is the word gangari, Sheng for 'being alert', which has been appropriated into Standard Swahili but expresses the same meaning as used in Sheng. I observed Swahili newscasters on different TV stations use it during news bulletins. The two examples therefore contravene the "purist" ideal associated with Standard Swahili.

From another point of view, the national ideal in Kenya is paradoxical in that the underlying notion of homogeneism presupposes that the use of the national language would generate prestige. However, the discussion in the previous sections demonstrated that speaking or embracing Standard Swahili in Kenya generates prestige in very limited contexts for various reasons. Instead, it is use of nonstandard language varieties such as Sheng that is highly rewarded by youth among peers. For example, the existing literature on Sheng reports that speakers embrace it as an alternative mode of communication, because they find the readily available languages such as Standard Swahili insufficient for their communicative needs, and for 
negotiating desired distinct social identities (Kiessling and Mous 2004; Wairungu

2013). This is well captured in a column in the Think Africa Press, June $12^{\text {th }} 2012$,

which states that:

Kenya's other languages, of which there are nearly 70, are assigned a subordinate position within this system. But Sheng appears to be defying this ideology by offering its users a contemporary identity distinct from those who use Standard English or Kiswahili. Through its simultaneous rejection and embrace of English and Swahili, Sheng breaks down the ethnic barriers often associated with language. Sheng therefore carries within it the possibility of unifying people of diverse ethnic backgrounds through its ethnically neutral underpinnings.

My observations agree with the arguments above in various ways. For example,

Kadonye, the student from Nakuru, reported that many students found Standard

Swahili hard, restrictive and also boring. Speakers who claimed that Standard Swahili

was hard and restrictive argued that it has complex grammar, especially the noun class agreement system which is burdensome. As a result, they claimed that it was hard for them to speak the language correctly. In comparison to Sheng, these speakers claimed that Standard Swahili is not flexible from a communicative point of view. For example, we were engaged in a discussion about boy-girl relationships in one allmale focus group in Nakuru, and I asked what students meant by their claim that Standard Swahili is restrictive. One of the students, Otis, responded that:

Unajua ulipoanza ulituambia tusikuwe restricted within anything, yaani tujiexpress venye tunaweza. Ndio maana information inatutoka tu yaani peke yake. Otherwise, ingekuwa eti ni kuongea Kiluhya hapa, ingekuwa ni vigumu na hata tungetumia lugha sanifu ya Kiswahili bado kungekuwa na matata, naona hivo. What I know is that Standard Swahili is not that flexible. If you want a certain information from me, I will give it to you but not full details. That is if you are restricting me to use a specific language. But if you want Sheng, then I will give you more details than expected. (Form Four focus group 4, 11/2011--Nakuru)

You know, at the beginning of this conversation, you told us that we should not be restricted within anything, that is, we should express ourselves however we feel most comfortable. That is why you see the information has been flowing freely... Otherwise, if we were using Kiluhya here, or even Standard Swahili we would still be experiencing communicative challenges, that is what I think. What I know is that Standard Swahili is not flexible. If you want certain information from 
me and you restrict me to use a specific language, I will give it to you but not full details. However, if you allow me to use Sheng, I will give you more details than you expected. (Form Four focus group 4, 11/2011--Nakuru)

The quotation above indicates that speakers of Sheng regard both ethnic languages and Standard Swahili as restrictive though in different ways. Also, they associate Sheng with communicative flexibility and freedom (see Chapter Six).

I then asked students in the focus group above to comment about why many of the students I had interviewed described Standard Swahili as boring. They reported that peers regarded those who spoke Standard Swahili and English most of the time as people who always wanted to show off. For example, Otis explained that he could not speak to his girlfriend in Standard Swahili because Sheng makes conversations "romantic." He elaborated that if a boy told a girl in Standard Swahili that amemkufia, 'he has died for her', the girl would think that he was lying. However, if they were speaking Sheng, the girl would feel good and regard the statement as true and romantic because the term amemkufia in Sheng means 'he is in love with her' (Form Four focus group 4, 11/2011--Nakuru). From an analytical point of view, Otis' example means that the term amemkufia has different meanings in Standard Swahili and Sheng. Thus, the speaker has to richly embed his/her Sheng in order to be heard as Sheng. So, Sheng is not just something to be uttered but to be performed as well. Other students in the focus group kept nodding their heads, indicating that they were in agreement with Otis.

Since romance among students cuts across ethnic lines, I found Otis' claim that Sheng makes conversations "romantic" quite interesting, because it challenges the role of Standard Swahili as a national language. Specifically, Standard Swahili is the medium that speakers from different ethnic backgrounds should use to 
communicate and express their feelings. For this reason, I asked Otis to comment further about the "romantic" nature of Sheng. He explained that if a boy spoke Standard Swahili or English to a girl, the girl would think that Otis was pretending or showing off. As such, boys speak Sheng to impress girls. However, Otis cautioned that girls usually took offence if wooed in their ethnic language, hence he would not expect that any guy would do it. In an attempt to elaborate why peers prefer Sheng over Standard Swahili, ethnic languages and English in their socialization, Otis emphasized that girls regarded guys who mixed languages as "trendy." He then sought to share some personal experience about how he was once ridiculed by a female classmate for addressing her in Standard Swahili. He said:

Wacha nikupatie perfect example, mimi nishaandika text kwa skirt fulani hapa, enyewe chenye alinijibu, mimi sikuamini. Nilimwandikia ati, nilikuwa najaribu kuisinuate my point. But alikuwa anajaribu yaani anaassume haelewi lakini anaelewa. Anataka tu yaani niache kucircumlocute nihit the nail on its head. Sasa mimi nilimwambia aje, ati wacha kunivalia miwani. Unajua kitu kama hio ni semi kwa Kiswahili na ni lugha sanifu. Yaani alinijibu mpaka nikaaibika. Ati niwache kujichosha na Kiswahili; Kiswahili ishadie. Yaani tumeshamurder paper ya Kiswahili. Sasa unaona inabidi turudi kwa Sheng. Kiswahili ni ya paper peke yake. Tena unajua Sheng, yaani saa kaa sisi tuende mahali sii watatu tuambiwe eti wee ongelesha vijana. Ниуи akuje aongelee tu English hivo, atachukuliwa eeh huyu kijana anaringa aje na hiyo Ingo yake? (Form Four focus group 4, 11/2011--Nakuru)

Let me give you a perfect example, I sent a text message to one of the girls in this school and I didn't believe the response I got. I had written to her trying to insinuate my point. But the girl pretended that she didn't understand; but she did understand. She wanted me to stop circumlocution and hit the nail on its head. I had told her in Swahili, 'acha kunivalia miwani'. You see that is an idiomatic expression in Standard Swahili. That is, the way she answered made me feel embarrassed. She told me to stop tiring myself with Swahili; the Swahili paper is already gone! That is, we have already done the Swahili exam. You see I had to return to Sheng. [Standard] Swahili is for examination purposes. In another instance, if the three of us went somewhere to address the youth and one of us used English, the youth would perceive that as a show off. (Form Four focus group 4, 11/2011--Nakuru)

I recorded similar sentiments from some students in Mombasa who referred to

Standard Swahili as "Kiswahili cha Shule", 'School Swahili” or "Kiswahili cha

Kitabu”, 'Textbook Swahili'. Also, some teachers in Nakuru and Mombasa 
reported that students did not regard English and Standard Swahili as languages for socialization purposes. Instead, these teachers claimed that students associated Standard Swahili and English with academic or class work and to some extent perceived them as either mere subjects of study or as languages for answering exam questions.

Some teachers also gave sentiments that agreed with Otis' claim that Sheng is "romantic." For example, Mr. Mwungwana, the teacher from Nakuru reported that young people used Sheng when having intimate talk in love relationships. He explained that they felt more "comfortable" using Sheng to tell their friends of opposite sex about their intimate feelings, and mentioning taboo words which would otherwise sound "disgusting" if said in other languages like Swahili, English or ethnic languages. For example, instead of young men saying that "I will not impregnate you,"Mr. Mwungwana told me that they would say, "sitakuweka ball”, 'I won't put a ball in you'. Also, instead of saying, "I will use a condom when making love," he told me that they would say, nitatumia socks 'I will use socks' (Mr. Mwungwana 10/ 2011, casual conversation). I argue that speaking Sheng is a performance that gives speakers the immunity to mention things considered obscene or nasty by adults. This immunity comes from the assumption that since adults do not understand Sheng, then it is safe for youth to talk nasty even in their presence.

Another motivation for speaking Sheng is that peers consider a person who is competent in Sheng as highly knowledgeable and up-to-date. For example, Mr. Kagogo, a teacher from Nakuru, reported that, "young people usually perceive someone who speaks Sheng as having mastered other languages like English and 
Swahili, but has made a choice to use Sheng” (Mr. Kagogo 10/2011, casual

conversation). This suggests that speaking Sheng marks the speaker as multilingual, which is highly valued among peers.

In Mombasa, students in a focus group told me that young people spoke "slang," and defined it as a kind of Kiswahili with specialized terms whose use is limited to small groups of speakers. These students then gave Sheng as an example of slang. One of them, Tinga, said that youth speak slang for various reasons including:

Desire to keep up with this generation. Sometimes if you don't speak slang, you don't seem like the youth of this time. You see slang changes with time. It is not something that is permanent. Like what was before several years, yah that kind of slang, and today is totally different due to new technologies which have introduced new names and vocabularies in the list, yah! And also new styles of life; new clothes and all that stuff has made changes in slang. So, if you don't identify with slang it means you don't identify with the new stuff; the new technologies, the new fashions and you are so much behind, very basic and non-developed. (Form Four focus group 1, 01/2012--Mombasa)

The sentiments expressed by Tinga and Mr. Kagogo above agree with the existing literature on Sheng in various ways. According to Samper (2002: 160), "Sheng makes one popular because you get noticed quickly and is more prestigious to speak because it makes you look modern." Some students in Nakuru and Mombasa reported that peers regard individuals who speak Sheng or lugha ya mtaa, and dress uniquely as having swag. According to Tinga, swag refers to an individual's or group's unique way of engaging in a social practice. These may be ways of speaking, styles of walking, dressing or hair style. Speakers appropriate these aspects of swag from the global media but give them local meanings (Form Four focus group 1, 01/2012-Mombasa). (See appendix C and D.)

From another point of view, both students and teachers in Nakuru expressed sentiments which suggested that speakers of Sheng embraced it because it helped them transcend ethnicity, an observation that agrees with the existing literature (e.g., 
Githiora 2002; Samper 2002). As mentioned at the beginning of this chapter, there is a strong connection between language and ethnicity in Kenya, especially among the adults. However, many young people, especially those in the urban areas, perceive ethnic identity and ethnic languages negatively. They associate them with rural life, older generations and tribalism from which they seek to distance themselves. For example, in one of the focus groups in Nakuru, I sought to know whether there were students who spoke ethnic languages in the school, and what peers said about them. One of the students, Eduba, responded:

Eeh, kuna wasee hutumia. Lakini mi enyewe na si kwa ubaya. Mi wasee wenye naonaga wakitumia hiyo lugha, [tsk], tuseme ni wale wasee wanatokaga huko ndani ndani yaani, yaani mashamba mashamba hivi. Unapata hata ukienda Mtaani unapata huyu msee anabonga hiyo lugha ya mama. Wakiwa na wazazi, masiblings. Saa anakuja anaiapply pia hapa. Lakini daro hawezi iapply. (Form Four focus group 2, 11/2011--Nakuru)

Yes, there are peers who use ethnic languages. However, and I don't mean to offend, the people I see using those languages [tsk], are those who come from the interior. I mean in the countryside or rural areas. You will find him/her using mother tongue with parents and siblings in the estate (neighborhood). Then s/he comes to use it here in school. However, s/he cannot use it in the classroom. (Form Four focus group 2, 11/2011--Nakuru)

There are several reasons why Eduba associated peers who use ethnic languages in school with mashamba 'country side'. From one point of view, he suggested that such peers lacked some exposure in life. This is because ethnic languages are rarely used in interethnic interactions. Instead, people either mix different languages in their conversations, speak Sheng or informal varieties of Swahili. In fact, Mr. Mwungwana, the teacher from Nakuru, had told me in another context that mixing of languages in a conversation is an indicator that the person has lived or has been exposed to cosmopolitan environment (Mr. Mwungwana 10/ 2011, casual conversation). It is in this context that speakers of Sheng label peers who lack such exposure as washamba. I recorded similar sentiments from students in Mombasa, who equally labeled peers 
who spoke ethnic languages in public as 'primitive', which they elaborated to mean washamba (Form Four focus group 2, 02/2012--Mombasa). ${ }^{54}$

Since ethnic languages in Kenya are generally associated with the ethnically homogenous areas, some students in Mombasa and Nakuru told me that speakers who used these languages among peers were labeled tribal and exclusionist. For example, a student in a focus group in Nakuru reported:

Watu hufikiria wewe ni mtribalic ukiongea lugha ya mama sana. Na watu wengine wanafeel like you are talking something yenye hutaki wasikie. Meaning hiyo kitu ni mbaya. Saa watu wanachukulia hivyo. Na tena unaweza kuja upate wewe huelewi lakini watu wanaongea unacheki enyewe ni kaa ni wewe wanaongelea juu hushiki. (Form Four focus group 2, 11/2011--Nakuru)

Some people think that you are tribalistic if you speak ethnic languages more often. Others feel like you don't want them to know what you are talking about suggesting that it must be something negative or bad. People usually think that way. And also, you might find people talking but since you don't understand their language, you start thinking that they are talking ill about you. (Form Four focus group 2, 11/2011-Nakuru)

In my earlier observations, some teachers and students in Nakuru had told me that Sheng speakers used it for passing in-group secrets. As such, I sought to know from the focus group above whether if a Sheng speaker found peers speaking a variety of Sheng that s/he did not understand would think that these peers were talking about him/her. Eduba responded that:

Obviously huwezi. Ukikosa kushika shika, hapo shida ni wewe. Sheng haijajificha sana isipokuwa wenye hawaongeangi sana. (Form Four focus group 2, 11/2011--Nakuru)

Obviously you cannot think so. If you do not understand, it's your problem. Sheng is not that secretive unless you are one of those who seldom speak it. (Form Four focus group 2, 11/2011--Nakuru)

Msud's and Eduba's responses above problematize the notion of "exclusion" in communication, because they suggest that the kind of "exclusion" exercised by use of Sheng is different from that which occurs when speaking ethnic

\footnotetext{
${ }^{54}$ As mentioned in Chapter One, the label washamba has multiple nuances of meaning.
} 
languages. It is also interesting to note that speakers of Sheng blamed peers who felt excluded in Sheng conversations, arguing that Sheng is not very exclusive. However, they took offence against peers who spoke ethnic languages, claiming that they felt excluded and sometimes feared that negative or bad things might be said about them. They therefore charged that speakers should not use ethnic languages during peer interactions.

Given that Sheng draws some of its linguistic material from ethnic languages which are associated with "country," exclusionism and "tribalism," I decided to find out how it helps speakers to transcend ethnicity. Some informants, both teachers and students, told me that speakers of Sheng encounter linguistic materials derived from ethnic languages when they have already been appropriated in Sheng. For example, Mr. Mwungwana, the teacher from Nakuru said:

Since most cosmopolitan neighborhoods in Nakuru are ethnically diverse, children usually learn the language commonly used in the area before they learn the language of their parents. It is therefore common for these children to pick words from peers and use them even before they know their source languages, e.g., many children in cosmopolitan Nakuru use the Kikuyu word Ngai [God] in their conversations but they don't speak Kikuyu. (Mr. Mwungwana10/ 2011, casual conversation)

Similarly, a student from Nakuru, Kadogo, reported that Sheng incorporates linguistic material even from ethnic languages that speakers do not know. However, when these words are introduced in Sheng, interlocutors are able to decipher their meanings possibly by studying the context of use or by asking peers. This means that speakers learn the meanings of these words and embrace them but without their ethnic connotations. These sentiments are captured in the extract below:

MW: I understand there are students from different ethnic backgrounds in this school, what is the main language of communication among the students? Tell us Kadogo.

Kadogo: Sheng. Sheng because it combines may be I will say every ethnic group. Because you will hear a Kikuyu word somewhere but it is Ok with everyone. Even a 
Kalenjin knows what it means. Because when you fix that, like now for money, there is this ngiri, they say ngiri. If you say ngiri to anyone, they will know it's a thousand. One thousand shillings is ngiri to everyone. $\mathrm{Na}$ ['and'] ngiri is in Kikuyu. Then at one time or another, you will hear another word that you don't know really but come to Sheng, you know what it means. But when you trace its background, it's from another language. So, it's like that. It's a combination and yet everyone understands it. (Praise and Worship focus group, 11/2011--Nakuru)

As explained in Chapters Two and Six, speakers of Sheng sometimes

manipulate the meanings of borrowed lexical items before they are used in Sheng, and this dissociates them from their source languages. In the case of words borrowed from ethnic languages, such semantic manipulation removes the ethnic connotation that would otherwise be considered offensive or exclusive among peers. For example, student Odusoh from Nakuru introduced earlier, reported that, "Sheng draws material from various languages such as Kikuyu, Dholuo and English, and then Swahilizes them to make them Sheng. He gave a specific example as shown in the following extract:

1. MW: Kwani Sheng huitwa Sheng ikicombine lugha gani? [...]

2. Odusoh: Yaani ni ile unapick word, for example kama kwa kiluhya, unapick word yaani kama Shipanya unakuja unaiswahilisha halafu inakuwa Sheng. Kama ni Kikuyu, unapick word tu,[...] Yaani unapick word kutoka kwa yenye unajua tu and then unaiweka ikuwe Sheng. [...]
1. MW: So, Sheng is called Sheng when it combines which languages?

2. Odusoh: That is, you pick a word, for example from Luhya, you pick a word like Shipanya and Swahilize it and it becomes Sheng. If it is Kikuyu, you just pick a word, [...] you just pick from the one ['language'] that you know and then add it to become Sheng $[\ldots]$

(Form Four focus group 2, 11/2011--Nakuru)

At the level of language choice, Sheng enables speakers to transcend ethnicity because it is not associated with any ethnic community in Kenya. Thus, speakers from different ethnic backgrounds feel comfortable to use it when communicating with peers. For example, I asked Mr. Mgema, the teacher from Nakuru, whether besides language, Sheng had other cultural elements such as dressing or manner of walking 
that identified its speakers. He interpreted my question to mean whether Sheng is associated with a particular ethnic group and responded as follows:

It doesn't because it cuts across all kinds of communities. So, you will find a Kalenjin speaking Sheng, you will find a Luhya speaking Sheng, you will find a Borana speaking Sheng so long as they are here in town. So it doesn't seem to have any culture as I would say. (Mr. Mgema 11/ 2011, interview)

Several other teachers in Nakuru expressed similar sentiments. For example, Mr. Muna referred to Sheng as a glue of the youth, claiming that Sheng would enable a young Kenyan from Kisumu (Western Kenya) to travel to Mombasa (South Eastern Coast) and relate to coastal youth with a lot of ease despite their ethnolinguistic differences (Muna 10/2011, interview). ${ }^{55}$

Contrastingly, ethnic differences often affect social interactions among adults, especially in situations where some people would switch to their ethnic languages rather than speaking Swahili including the standard. For example, I asked several teachers in Mombasa and Nakuru whether the 2007 post-election violence affected teachers' and students' social relationships and their patterns of language use.

According to Mr. Mpoa, the teacher from Nakuru, teachers' social relationships were affected significantly. The school did not have teachers from certain ethnic communities like the Kisii before the 2007 post-election violence. They joined the school after they were evicted from areas like Kericho, where their communities were perceived as outsiders by their ethnic rivals. Also, some of the teachers who were in this school before the post-election violence but were from the ethnic communities perceived as outsiders in Nakuru - like the Luo were attacked (Mr. Migingo 10/2011,

\footnotetext{
${ }^{55}$ The claims by Mr. Mgema and Mr. Muna are informed by the language ideology which associates Sheng with all youth in Kenya. I argue that this is not true because my study established that many young Kenyans in Mombasa associate with lugha ya mtaa with some claiming that it is different from Sheng.
} 
casual conversation). Some of them transferred to other regions while others left temporarily and returned after the violence. From then on, Mr.Mpoa told that teachers had regrouped along ethnic lines where they openly conversed in their ethnic languages. Specifically, he said that teachers from the Kikuyu and Kisii communities spoke in ethnic languages most of the time even in the presence of other teachers, especially in the teachers' lounge (Mr. Mpoa 10/2011, casual conversation).

However, Mr. Mpoa said that students' social relationships and language use patterns were not affected by the 2007 post-election violence because of several factors that included: 1) the school is cosmopolitan, hencemany students were from mixed ethnic backgrounds; 2) most of the students were not fluent in any of the parent's ethnic languages; and 3) they do not form their friendship networks along ethnic lines. As such, Mr. Mopa said that it was rare to hear students communicating in ethnic languages such as Kikuyu or Dholuo like teachers. Instead, they always used Swangenglish, which he described as a mixture of English and Swahili. He then claimed that students were more "nationalistic" in their choice of languages for communication as opposed to their teachers who spoke ethnic languages most of the time (Mr. Mpoa 10/2011, casual conversation). Mr. Mpoa's claims indicate that he subscribed to the colonial language ideology which regards ethnic languages as a threat to national unity.

I recorded similar sentiments from Mr. Naibu, the teacher from Mombasa introduced earlier. He claimed that children nowadays are not as attached to their ethnic communities as it was the case during his time, when he and his peers used to engage in traditional cultural practices such as circumcision songs. Mr. Naibu said that such cultural practices cultivated a strong connection with their ethnic languages. 
As a result, he enthusiastically reported that there were some words in Luhya, his ethnic language, which made him feel good when he pronounced them. In this context, he told me that his ethnic language was the best medium for expressing his inner emotions. This confirms his claim that his generation is strongly tied to their ethnic languages. Contrastingly, he observed that most children nowadays do not even know or speak their ethnic languages, especially those from mixed ethnic marriages. Instead, Mr. Naibu claimed that, "Kids nowadays try so much to avoid being associated with ethnic background, [indicating that]...children's level of identity is changing to a future where children will be more patriotic to Kenya than to their communities" (Mr. Naibu 01/2012, interview).

Besides transcending ethnicity, speakers of Sheng reported that they also used it to negotiate a sense of belonging among peers, which would not be possible if they spoke the officially sanctioned Standard Swahili. As these speakers seek to associate with desired social groups, they at the same time seek to distance themselves from others. One of these social categories is the older generations because they often stand in opposition to the values and practices of the youth. For example, after I found that many students were critical of the officially sanctioned languages, I asked students in a focus group in Mombasa whether there was a particular language that was popular among the youth in Kenya. Tinga, introduced earlier, responded that it was Sheng and emphasized that speakers mainly used it to communicate ingroup secrets and to exclude older generations from their conversations. According to Tinga: 
Mostly, the language of the youth in Kenya is that one which is not understood by the old. And the Sheng, by the way Sheng can be Sheng but differs from one city to another, from one town to another. The Sheng in Mombasa is not the same Sheng in Nyahururu. These two are different but the major purpose is to hide the information from the old. That is where we converge all of us. We do not want them to know. We find it boring, you know, that gap eeh! We find it boring to associate ourselves with the old because we are so vigor [sic] and energetic. (Form Four focus group 1, 01/2012--Mombasa)

In summary, the discussion in this section has demonstrated that Standard Swahili and various youth vernaculars such as Sheng are in an antagonistic relationship. In the context of national language, I foregrounded Sheng in the discussion because its speakers envision it as the future national language of Kenya making its antagonism with Standard Swahili the most pronounced. I have argued that speakers of Sheng find Standard Swahili inadequate for serving their communicative needs and for negotiating desired distinct identities. Also, speakers of Sheng have a language ideology which conflicts with the school language policy. That is, while the school encourages the students to embrace Standard Swahili as informed by the notion of homogeneism, speakers of Sheng disapprove of peers who speak Standard Swahili and label them washamba. In this regard, language competence among Sheng speakers is not measured by the performance of the standard but the ability to draw from the many languages in the speaker's repertoire. The same happens among many youths in contemporary urban Africa (Makoni et al. 2007).

In addition, the discussion also demonstrated that it is use of Sheng rather than Standard Swahili which generates more social rewards among many young Kenyans. My research findings therefore confirm the existing literature (e.g., Migunda-Attyang 2007) that it is Sheng rather than Standard 
Swahili, which serves as the national language for these speakers in actual practice. In this context, I became interested in knowing what the enthusiasts of Sheng thought about its future in Kenya. I focus on this issue here below.

\subsubsection{The future of Sheng in Kenya}

I asked students in one mixed-gender focus group in Nakuru whether they would be speaking Sheng in the next five years or so. One of them, Mailinne, responded in the affirmative. He stated that if even his elderly mother spoke Sheng, there would be no reason for his generation to stop using it (Form Four focus group 1, 11/2011--Nakuru). He elaborated that parents were learning Sheng in order to engage the youth. Also, he observed that Sheng had become the language of business in Kenya, because unlike a few years ago, it was now common for businessmen to employ Sheng words in their conversations, some of which were new to Mailinne. He argued that both traders and clients had to embrace Sheng and constantly update it in order to do business successfully. Mailinne's sentiments were supported by a female student called Mpole from the same focus group who reported that Sheng had been embraced by many people ranging from children to youth to parents. According to Mpole, Sheng is spoken by $70 \%$ of Kenyans, making it the most idely spoken language in Kenya.

In support of Mpole, Mailinne added that the population that spoke English in Kenya was very small. Also, young people only spoke ethnic languages when interacting with parents who did not understand Sheng. With regard to Swahili, Mailinne said that even though many people spoke it, the majority often introduced Sheng words into their conversations. This might explain why Mpole and Mailinne 
came to the conclusion that Sheng is the dominant language (most widely spoken)

among many people in Kenya, and that their generation will still be speaking Sheng in the next five years (Form Four focus group 1, 11/2011--Nakuru). ${ }^{56}$

Mailinne's claim that more parents are nowadays embracing Sheng than before was also shared by some teachers both in Nakuru and Mombasa. For example, I asked Mr. Mgema, the teacher from Nakuru, to comment about adults who spoke Sheng. His response was:

I believe every person has their own reasons. And you know it depends on the person. There are people who are very intertwined in their families with their children. So they go to the level of the children to be able to communicate with them. So in such a home, you find that even the parents are speaking Sheng. You know the children are very powerful people, very very powerful people. For example, if you are going to a home, you might find that everybody in that home calls grandmother сиси because the kid is the one who calls her cucu. So, now they have learnt the language of the children. If a kid comes up and instead of saying radio, he calls it deko, then you will find many people calling it deko. (Mr. Mgema 11/ 2011, interview)

Some informants also claimed that Sheng has a bright future because several new forms of media produced in Sheng have emerged in the last few years. For example, sudents Mpole and Mailinne mentioned above emphasized that the new media targeted the youth who were the majority in Kenya's population demography. These media included magazines such as the Young Nation and comics such as Shujaaz, circulated together with the national newspapers Sunday Nation and The Standard respectively. Mailinne and Mpole also emphasized that the newly emerged Sheng publications "even have a dictionary." The dictionary they referred to here was a glossary provided on the last page of Shujaaz magazine. Mailinne's and Mpole's

\footnotetext{
${ }^{56}$ These speakers used the term "dominant" to mean the most widely spoken language in Kenya, rather than the language associated with political power.
} 
emphasis on the existence of a dictionary seems informed by the language ideology that a dictionary gives a language some legitimacy (Lippi-Greene 2012).

The claims above that Sheng has a bright future in Kenya, and its use in the media has increased have also been reported in the media. One article states:

A growing number of publications such as comic books Shujaaz and Straight Talk or the literary journal Kwani?, for example, are written entirely in Sheng or feature articles in Sheng. Straight Talk, produced by and for young people, addresses topics such as sexual abuse, pregnancy, homosexuality, and gender inequality while Shujaaz, a comic book launched in 2010, aims to empower young Kenyans with information, ideas and the motivation they need to become active participants in issues such as development and community cohesion. (Think Africa Press, June $12^{\text {th }}$ 2012)

In addition to the written forms of media, Sheng has also penetrated the national radio and TV stations, which were previously perceived as a reserve of Standard Swahili and English. For example, several TV and radio stations have introduced programs in Sheng targeting the youth. They include Beba Beba and Raiyaa aired on Citizen TV and Churchill Show aired on NTV. In addition, the current crop of secular and religious musicians, televangelists and even politicians has increasingly embraced Sheng with the aim of reaching the majority of young Kenyans. For example, Halliday (June 12 $2^{\text {th }}$ 2012), writing for the Think Africa Press reports that:

By the 2000s, however, it was not only the young Hip Hop artists and listeners who were using Sheng. During the run up to the 2002 elections, Mwai Kibaki, leader of the National Rainbow Coalition (NARC), addressed his audience with the phrase "we are unbwogable!" The Sheng word "Unbwogable"- meaning unshakeable - was the name of the popular hit by Hip-Hop group Gidi Gidi Maji Maji. "We are unbwogable" became NARC's campaign slogan and soon Kenyans, old and young, incorporated "Unbwogable" in their everyday dialect.

Following these developments, the number of Sheng speakers has been on the rise.

Sheng has also spread from Nairobi, its perceived origins, to other major towns and rural areas. Ultimately, use of Sheng (to some extent) is no longer dictated by biological age. Neither is it confined in one geographical or social space. Instead, 
some adults, perhaps because of the nature of their occupations among other reasons, have embraced Sheng in their daily operations. Among these groups are street vendors and pop musicians. Some people's attitudes toward Sheng have also changed to the positive. For example, non-speakers like Mr. Mgema, the teacher from Nakuru, recognize the many roles that Sheng plays among its speakers. Even some speakers who had earlier claimed that they spoke Sheng to distance themselves from the older generations evaluated adults and figures of authority who speak Sheng positively. For example, students from both Nakuru and Mombasa such as Mailinne, Mpole and Tinga, reported that youth in Kenya appreciated such leaders because their use of Sheng projected them as people who both appreciate the youth and understand the issues affecting them.

Besides the fact that the society's general attitudes that Kenyans express toward Sheng and its speakers are becoming more positive, I also observed that speakers of Sheng are not as passive in their language choices as they were socially constructed by figures of authority. For example, I asked students in Tinga's focus group in Mombasa about whether there is a particular language that is generally considered popular among the youth in Kenya. They identified that language to be Sheng and added that speakers of Sheng made informed language choices depending on the context. Speakers also understood the consequences of not using the required language in any given situation. Specifically, Tinga told me:

The most popular is that casual language-the Sheng, which is spoken in an environment which is not serious, yah! But when it comes to serious issues, if you want your English to be so much respected or your Kiswahili to be so much high, you know! You need to improve it according to the occasion. Sometimes if you speak Sheng in an official function eeh, you know also youth are not that stupid, they have also learned that there is an official language and also casual language. They will think that you are not well informed in terms of language. In fact they will think that... they might even boo you because they will think that you are out of senses. (Form Four focus group 1, 01/2012--Mombasa) 
In conclusion, this chapter has examined the contested status of Standard Swahili as the national language of Kenya. I have argued that though the government's choice of Standard Swahili was informed by the desire to "reafricanize" Kenyans, and break from the "chains" of colonization by using a local language (Sifuna 1990), some Kenyans claim that Standard Swahili is a foreign language just like Arabic, Gujarati or English. Similarly, though the government chose Standard Swahili because of its perceived ethnic neutrality, speakers of Kimvita contest that it is a foreign variety and does not fit well as the national language of Kenya. In this context, speakers of Kimvita perceive Standard Swahili as a threat to their ethnic identity, and feel that embracing it would lead to cultural domination because they regard Kimvita as their cultural reservoir.

From a practical point of view, and despite intensive efforts in schools to promote Standard Swahili as the national language, many Kenyans from different ethnolinguistic backgrounds do not employ it in their day-to-day interactions. Instead, educated adults mainly employ English while those in the rural settings employ ethnic languages. Even those who claim to speak Swahili either refer to contact varieties which are closer to Sheng than to the standard, or to the coastal varieties such as Kimvita. In this regard, the chapter demonstrated that Kenyans in general have a very low regard for Standard Swahili, suggesting that the language enjoys the status of national language only in theory. This contrasts with the case of Standard Swahili in the neighboring Tanzania, where scholars claim that it has been more successful both as a national and official language. Even though that is the case, the chapter highlighted that Standard Swahili in Tanzania is still looked down upon in favor of 
English as witnessed during beauty pageants. Also, contrary to the belief that Tanzanians always speak "pure" and Standard Swahili, the chapter established that they also mix languages in their conversations and that there also exist lugha za mitaani (street languages), especially in the urban areas.

However, the major difference between the Kenyan and Tanzanian cases is that while Tanzanians reprimand individuals who deviate from the standard in formal or official contexts, Kenyans easily get away with speaking Swahili “incorrectly.” In the case of my study, some informants made claims that suggested that Kenyans in general are usually more worried about speaking English "correctly." This is one of the reasons why English continues to dominate Standard Swahili even in school settings where the two languages are in principle supposed to be embraced equally.

With regard to the link between language and authority, the discussion demonstrated that the way people in influential positions such as politicians, government officials and the police use language, and the identities that they project as they interact with the citizens, greatly influence peoples' language attitudes toward the language in question. A good example is how colonial administrators embraced Ki-Settla and used it as a tool for oppressing the Africans, and this negatively affected the attitudes that Africans had toward Swahili in general. The same is true with police in Uganda and Kenya. The police in Kenya employ a variety of Swahili that resembles Ki-Settla from a grammatical point of view. They employ this variety in ways that project them as harsh and arrogant. Consequently, Kenyans in general associate people who speak that variety of Swahili with arrogance.

Finally, Standard Swahili is in an antagonistic relationship with various nonstandard language varieties such as Sheng and lugha ya mtaa. Speakers of Sheng 
in particular have gone a step further to envision it as Kenya's future national language. At the same time, some figures of authority such as teachers and parents who were previously opposed to Sheng nowadays acknowledge that Sheng performs significant social functions among speakers such as transcending ethnicity. This suggests that peoples' attitudes toward Sheng are changing to the positive. Sheng has also spread to domains which were previously associated with Standard Swahili. All these factors suggest that Standard Swahili is continuously losing purchase among many Kenyans, hence there is a need for government to review the national language policy. Such policy review should acknowledge that language competence among the youth, who are the majority in Kenya, is measured by the ability to innovate from different languages rather than adherence to the use of the standard language. As such, the prevalent multilingualism in Kenya and the wider post-colonial Africa will no longer be perceived as a problem, but a communicative resource. In the next chapter, I focus on the domiant official language ideology arguing that it is paradoxical in various ways. 


\title{
Chapter Five
}

\subsection{Nonstandard Language Varieties, Education and the Paradoxical Official}

\section{Language Ideology in Kenya}

\begin{abstract}
We [Kenyans] are not so keen on the kind of Kiswahili we speak because the perception is that when you speak English, it means you are modern, you are civilized, you are a man or a woman of status and all that. And when you speak in Kiswahili and you break it; it is normal and it shows that you are a man of status, you are civilized and that is why you don't have a command of Kiswahili. That you are high up there, Kiswahili is down here you may not have a command of it. So, it is not a big issue. So, it is a hurdle to leave your comfort up there and come down here to speak good Kiswahili. So, that is it. (Mr. Seneta 03/2012, interview)
\end{abstract}

This chapter focuses on the paradoxical nature of the dominant official language ideology associated with the use of English and Standard Swahili in schools and wider Kenyan society. As noted in Chapter Three, Kenya at independence inherited colonial structures where English was socially constructed as the language of prestige and power. Therefore, the government sought to break from the chains of colonialism by promoting one of the local languages, to be used in conducting formal government transactions such as parliamentary debates and classroom instruction (Mazrui and Mazrui 1995). Even though this goal took many decades to be achieved, the government eventually elevated Standard Swahili to the status of an official language in 2010 (National Constitution 2010; Standard Newspaper, August 30 2010).

However, my field observations indicate that this development did not change the perceptions that many Kenyans had toward Standard Swahili. For example, recall from the quotation at the beginning of this chapter that many Kenyans regard English as more official and important than Standard Swahili, and are therefore more worried about speaking the former more "correctly" than the latter. As a result, English continues to enjoy its colonial and historical hegemonic status. Also, despite schools' 
emphasis on the value of English and Standard Swahili as official languages, not all students have embraced them and the associated identities. Instead, many have embraced nonstandard language varieties such as Sheng and lugha ya mtaa for various reasons. These include negotiating desired distinctiveness and "freedom" from the confines of the local cultural frameworks (see the next chapter).

Teachers and other figures of authority criticize students' embrace of these nonstandard language varieties, especially Sheng, arguing that they interfere with students' mastery of Standard Swahili and English, and their overall performance in national exams (Githiora 2002; Momanyi 2009). Also, perhaps because modern technology and western 'pop' culture dominate the discourse of these nonstandard language varieties, my field observations indicate that the various authority figures perceive speakers as culturally eroded and having discipline problems. Consequently, these authorities argue that schools should ban these language varieties, and promote the officially sanctioned Standard Swahili and English. The current linguistic situation in Kenya lends itself to the following questions: 1) what identities are promoted by the dominant official language ideology? 2) Are these identities limited to the use of "correct" language or do they also cut across other aspects of students' behavior such as manner of dressing? 3) What practices contribute to the hegemonic status of English to the detriment of Standard Swahili, the other official language? 4) Do all teachers oppose students' use of nonstandard language varieties such as Sheng and lugha ya mtaa, or are there some who regard them positively? 5) Do teachers utilize ethnic languages when teaching, or do they also perceive them as impediments to the learning process? 6) Are efforts by schools to eradicate the various nonstandard language varieties successful in actual practice? I engage these questions in the 
remainder of this chapter, starting with the notion of "standard language ideology" and associated identities.

\subsection{0. "Standard language ideology" in Kenya and associated identities}

The term "standard language ideology" has been applied to beliefs about standard languages that are common in Western societies. According to Lippi-Green (2012), it refers to a socio-political bias towards an idealized homogenous language that is associated with the speech of the dominant (upper middle) class. Institutions such as schools and mainstream media promote the use of the standard and prescribe that it is the "best" variety of language to use in all situations. In the process, these institutions socially construct the standard as a "one- size-fits-all" medium of communication that is "pure", "correct" or "free of errors" (Alim 2004; Lippi-Green 2012; Pullum 1999). Lippi-Green (2012) adds that the standard is usually protected by individuals who make a living out of the concept. These include lexicographers, grammarians and language teachers. She says that these "experts" operate on the assumption that "something as important as language cannot be left to itself: normal people are not smart enough, not aware enough, to be in charge of their own language. There must be experts, persons in charge, structured authority" (Lippi-Green 2012: 60). In this regard, the said "experts" prescribe how the standard language should be written or spoken. These experts assume authority of judging other language users, employing parameters such as written exams that they have set themselves.

Speakers who embrace the standard are socially constructed as educated and more acceptable by the authorities, than speakers of nonstandard language varieties. Ultimately, this censorship endows the standard with some social value over other 
varieties. In educational contexts, for example, schools use the standard language as a yardstick for evaluating language competence, and measuring the worth of other languages. While use of the standard is rewarded, use of other language varieties is punished. However, "standard language ideology" does not entirely fit the Kenyan situation, as I will elaborate further below.

The notion of "standard language ideology" owes much to the work of Pierre Bourdieu. According to Bourdieu (1977), even though linguists claim that all languages are linguistically equal in their expressive and communicative abilities, languages have unequal symbolic value in the "linguistic market," which brings about social inequality between interlocutors. He defines a "linguistic market" as that particular situation in which linguistic exchanges take place. He argues that these marketplaces have implicit laws of production that are decisive for understanding what can and cannot be said at any point in time. In this context, one language variety, which he calls "legitimate language," may be elevated over others for socio-political reasons. Within this state of inequality, Bourdieu (1977: 652) argues that "a language is worth what those who speak it are worth." For example, he reports that a school "imposes the legitimate forms of discourse and the idea that a discourse should be recognized if and only if it conforms to the legitimate norms..." (Bourdieu 1977: 650). In this regard, school authorities prescribe that standard language is the "correct" and acceptable medium of communication, while disparaging nonstandard language varieties. Next, I focus on how the notions of "standard language ideology" and "legitimate language" articulate with the cases of English and Standard Swahili in Kenya. 


\subsection{1. "Legitimate language" and the politics of linguistic "correctness" in Kenya}

The cases of English and Standard Swahili in Kenya differ from that of English in the West, e.g., the U.S.A, in various ways. For example, there are several native (mother tongue) varieties of English spoken in the U.S., but it is only the "standard American English" which is considered "correct," hence "legitimate" in educational settings. Consequently, teachers in the U.S. schools correct "errors" related to the use of the standard such as grammatical features of nonstandard varieties of English like African American Vernacular English (AAVE) (Alim 1999; Labov 1972; Lippi-Green 2012; Rickford 1999). For example, Labov (1972), reports that some teachers in U.S. schools devote time and energy to correcting a small number of non-Standard English rules such as the double negative (also called negative concord), terming them illogical. This linguistic situation resembles the case of Standard Swahili in Kenya, where teachers correct mistakes associated with imperfect second-language learning, and also regard local native varieties of Swahili such as Kimvita as "errors." An example of this is the teacher from Mombasa, where Swahili is spoken natively, who said that a student wanted to say amekuja, 's/he has come' but instead said ankuja (the Kimvita way of saying it).

The other similarity between the case of English in the U.S and Standard Swahili in Kenya is that in the U.S. schools, Labov (1972: 230-31) argues that teachers who subscribe to Bereiter and Englemann's (1966) verbal deprivation theory, often hear AAVE speakers not as speaking a variety of English that is different from theirs, but also one that is a marker of primitivity and savagery. In the Kenyan context, recall that Mr. Naibu from Mombasa, said that his school associated students' use of mother tongue and Swahili with making noise, promoting violence and use of 
vulgar and abusive words (Mr. Naibu 01/2012, interview). Despite this resemblance, both "standard language ideology" and "legitimate language" do not fit well in the case of Standard Swahili for various reasons that I discuss below.

The evaluation of "correctness" in the case of English in Kenya differs from that of the U.S in that there are no competing native varieties of English. Also, the variety that Kenyan schools promote is the one found in textbooks, hence it is standard by definition. Some of the "errors" that teachers correct include grammatical mistakes such as verb tenses, mother tongue interference and problems associated with second language learning such as direct translation. In Nakuru for example, Mrs. Kiboko reported the case of one English teacher who asked students in her class to mention the English proverbs that they knew. One student raised her hand and said, "late, late, you will find the baby is not yours." Mrs. Kiboko explained that this student had literally translated the Swahili proverb, “chelewa chelewa utampata mwana si wako”, 'The early bird catches the worm' (Mrs. Kiboko 11/2011, interview). Some teachers in Mombasa also reported such cases of direct translation from Swahili to English. For example, Mrs. Munene spoke of a colleague who was passing by the Form One classrooms and overheard a female student reprimanding another saying, "stop sweeping above above!" This teacher found it amusing and called that student, seeking to know what she meant. The student explained in Swahili that she meant to say, “Acha kufagia juи juu”, 'stop sweeping superficially’ (Mrs. Munene 04/2012, interview).

Despite these differences between the linguistic situation in Kenya and in the U.S., some aspects of the notion of "legitimate language" and "standard language ideology" fit well in the case of English in Kenya. In the Mombasa school for 
example, recall that the language policy was revised from one that allowed students to speak Standard Swahili for one day in a week, to one that required them to speak English all the time. Also, teachers and students reported that the school had employed different measures at different times, to reinforce students' use of English. These included asking violators to write essays in English and score a certain grade, failing which they would be asked to write another one. The school had also devised a portable token such as a piece of wood, which a violator would pass on to the next one, so it would circulate for the whole day. Each violator was required to remember who passed it to him or her. At the end of the day, teachers would punish these violators by asking them to wash verandahs or classrooms. Most recently, the school devised a long ugly dress for violators to wear, which was meant to embarrass them as they walked around the school. According to Mrs. Munene, even though these measures "died" after a short period of time, they helped discourage students from speaking unauthorized languages, especially Kiswahili (Mrs. Munene 04/2012, interview).

Some teachers in Nakuru also emphasized the value of English over Standard Swahili. Mr. Nyachae, for example, argued that though both Swahili and English were important exam subjects, all other subjects apart from Swahili were taught and examined in English (Mr. Nyachae 11/2011, interview) ${ }^{57}$ He therefore took issue with the school administration, arguing that it had not put in place a language policy. As a result, he said that students spoke Swahili and other languages such as Sheng most of the time, a situation that he said had significantly affected their performance

\footnotetext{
${ }^{57} \mathrm{Mr}$. Nyachae is a former school Principal and had worked in different schools in different parts of Kenya before he sought to be deployed as a classroom teacher in Nakuru, following the 2007 postelection violence.
} 
in English exams. In this regard, he alleged that the school's lack of a language policy and rules to reinforce it, indicated that the school administration had given in to students' use of unauthorized language varieties.

According to Mr. Nyachae, students who always spoke Kiswahili, but made no effort to speak English had shortcomings. He felt that his school needed to emulate schools that performed well academically. He said:

You know what, in some schools, you know they even stress, they have more weight
in English than in Kiswahili and they have even introduced disks [a token to be
passed from one violator to another] to control the use of Swahili. And they say that
this language here, English, $99 \%$ of what you do will be in English...If you went to
Aluya High School. I happened to have gone there. Those students are good.
Everybody is talking very fluent English, everybody is very courteous, you see! If
they see you are a visitor, a student comes, "excuse me, may I help you," you know.
You feel good, you feel nice. But come here, the students want to knock you on the
corridor, yah. (Mr. Nyachae 11/2011, Interview) Mr. Nyachae's sentiment that there is a need to promote English more than Standard Swahili suggests that though both languages are official, some Kenyans regard English as more official, hence more "appropriate" or acceptable than Standard Swahili. This is one of the contexts where Bourdieu's (1977) notion of "legitimate language" does not fit well for Standard Swahili in Kenya. From another point of view, Mr. Nyachae's claim that “everybody in Aluya High School spoke fluent English" and that "everybody is very courteous" reveals the effects of "standard language ideology" among school authorities. At one level, Mr. Nyachae equated speaking English fluently with having good behavior, which in this case was defined by being "courteous" and "respectful" to visitors. At another level, he equated speaking English fluently with academic excellence and to some extent being 
intelligent. ${ }^{58}$ In this context, I argue that students who embrace the "legitimate language" (English in this case), earn social acceptance and symbolic rewards from authorities. Such rewards may come in the form of good grades on exams or direct compliments from teachers. For example, Mr. Mgema, the teacher from Nakuru introduced in the previous chapter, confesses in the extract below that he had a high regard for people who speak "good" English:

Personally, I appreciate people who speak English. Like I met a girl in, eh, Form One. I met her in the deputy's office and, eh, I asked something and she answered. And I said, eh that is very good English. I told her, keep reading, make sure that your language grows; is good. So, when I see people who speak good English, I am always excited about it. (Mr. Mgema 11/ 2011, interview)

According to Lippi-Green (2012), the various symbolic associations and rewards connected to the use of the standard language, socially construct it as the language of prestige, power and social mobility. However, as argued further below, use of Standard Swahili in Kenya is rewarded in very limited contexts. Besides, it is neither associated with the elite nor does it mark the speaker as either intelligent or educated. As such, competence in Standard Swahili does not guarantee social acceptance among authorities or social mobility. In fact, there are cases such as in the Mombasa school where use of any variety of Swahili, including the standard is punishable. From the foregoing discussion, I argue that there is a disconnect between peoples' embrace of English in comparison to Standard Swahili, the other official language. I now examine this disconnect.

\footnotetext{
${ }^{58}$ Note that the claim that students in schools that perform well academically do not speak Sheng is not true, based on my observations. However, it is relevant from the point of view of language ideology. For example, recall that some speakers had steered me away from the Nakuru school arguing that I would not encounter Sheng since that school is an academic giant. They instead referred me to the schools that perform poorly in national exams especially mixed-gender, day schools located in the low income neighborhoods. Another group of friends steered me away from Mombasa arguing that Mombasans speak Swahili, hence I would not encounter Sheng.
} 


\subsection{2. "Inequality between two equals": English vs. Standard Swahili in Kenya}

In an interview with Mr Seneta, a teacher of Standard Swahili from Mombasa, I asked him to comment on why Kenyans perceived the two official languages differently. According to him, the problems facing Standard Swahili emanated from general social attitudes towards the language. These sentiments are well captured in the quotation at the beginning of this chapter where Mr. Seneta says that when someone speaks English, Kenyans perceive him to be superior than if he had spoken Swahili. From the point of view of language ideology, I inferred from Mr. Seneta's sentiments that Kenyan society attaches a higher symbolic value to English than Standard Swahili. These sentiments echo the colonial language ideology reported in the previous chapter, where British settlers and the colonial administrators in Kenya looked down upon Swahili in general, arguing that for them to speak it "correctly" would be downgrading their status to the level of the "peasants," that is, Africans (Mkangi 1984; Mutahi 1979; Whiteley 1993). These paternalistic attitudes toward Swahili and Africans are comparable to the case of Spanish in the U.S. where middleclass, English-speaking Americans easily get away with speaking Spanish "incorrectly," while native speakers of Spanish are under severe pressure to speak English “correctly" (Hill 1998; Urciuoli 1996). In one specific context, Hill (1998: 682) says that, "[w]hile Puerto Ricans are extremely self-conscious about their Spanish 'accents' in English, heavy English 'accents' in Spanish are perfectly acceptable for Whites, even when Spanish speakers experience them as 'like a fingernail on the blackboard."”

According to Urciuoli (1996), this difference owes to the fact that "good" English is a highly regarded "symbolic capital" in the U.S. society. As a result, 
middle-class, English-speaking Americans look down upon Spanish and other language varieties; they do not see the need to speak them "correctly." However, the reverse is not true. Speakers of Spanish have to negotiate strategies for survival such as learning "good" English or pretending not to know their native language. Bourdieu (1977: 653) expresses similar sentiments, saying that, "the dominant class can make deliberately or accidentally lax use of language without their discourse ever being invested with the same social value as that of the dominated. What speaks is not the utterance, the language, but the whole social person."

A similar scenario exists in Kenya, especially in urban settings, where many people openly claim that they do not know their "ethnic languages" or Standard Swahili. Some parents even take pride that their children speak only English. For example, Mr. Seneta said that he knew speakers who were fluent in Standard Swahili but refused to associate with it in public settings. He told me that there were teachers who scored excellent grades in Swahili national exams when they were students and at some point taught the language as untrained teachers, but no longer liked speaking it in public or group settings. ${ }^{59}$ In This context, Mr. Seneta argued that such teachers contributed immensely to students' negative attitude towards Standard Swahili.

According to him:

The teachers; some of them have actually taught Kiswahili as untrained teachers and if you talk to them privately, some of them are very good in Kiswahili. But if you tell them to speak Kiswahili, they will tell you 'aah sijui ama [I don't know or] am not so competent in it'. But if you look at their Form Four results, Kiswahili was, may be, their best subject. And some of them, a good number of them, taught Kiswahili as untrained teachers before they joined university. If they taught Kiswahili, that means they are good at the language. So what happens? It is the society that fails us, but we [teachers] are also to blame. (Mr. Seneta 03/2012, interview)

\footnotetext{
${ }^{59}$ The term "untrained teachers" in Kenya refers to high school graduates who have not been professionally trained as teachers, but are hired locally by understaffed schools to supplement the professional teachers employed by the government.
} 
Even though Mr. Seneta stated that the whole of Kenyan society contributed to the challenges facing Standard Swahili there, he singled out the older generations, claiming that they carried the biggest blame, especially in situations where the language competed with English. In his own words:

The biggest challenge to the development of Kiswahili is those people who were born in the 60s and below; those born in the " 50 s and ' $40 \mathrm{~s}$. And a very big chunk of them come from that age bracket. They are the biggest letdown because they will tell you 'that was not part of us'. Yet they can speak. You know they speak! Some of them happen to be my brothers and sisters. They speak very good Kiswahili. When we speak, we speak. But they would tell their children not to speak Kiswahili and when you now push the discussion further, when we are the two of us is Ok but when we become a group, they want to speak English and claim that they never learned Kiswahili in school because it was optional. That is where the problem is. (Mr. Seneta 03/2012, interview)

Mr. Seneta's claims are true to some extent. Other informants like Mr. Nyachae, Mr. Mgema and Mrs. Munene, reported that they rarely spoke Standard Swahili because they did not learn it formally when they were students. They claimed that Swahili was an optional subject and only few students enrolled. Thus the Swahili they spoke was acquired from peer interactions. Teachers in this category emphasized the value of English over all the other languages that students spoke including Standard Swahili. They argued that students needed to take English seriously since it was the main medium of educational instruction and excelling in the subject would lead to good career prospects even outside Kenya.

From another point of view, Mr. Seneta's claims suggest that, even though students have their own shortcomings in using and embracing Standard Swahili in their communication, there is a stark disconnect between language use patterns within the school and in the outside society in actual practice. He said for instance:

In fact, it is the society that changes these students. When they go out and mix with the society. I wish you could know how students react when a fellow student makes a grammatical mistake in Kiswahili in class. One, they will laugh and tell him that that 
is not standard Kiswahili. You could have said this. They will correct the situation. They will say 'this is how it is said or this is how it is done. Not the other way round.' And, ah, that laughter alone is enough punishment for someone to break Kiswahili again. But when they go back to society, society tells them that that was academics, we are back to reality. So now that you have passed your grades, you have your ' $A$ ' in Kiswahili, that was in school, you can enjoy a soft landing. Here you can break the rules without any problem. We have no issues if you break grammatical rules. (Mr. Seneta 03/2012, interview)

Given the social rewards associated with the use of the officially sanctioned Standard Swahili and English such as good grades in exams, and promising career prospects after graduation, I sought to know more from Mr. Seneta about why students had not fully embraced the two languages. According to him, many students had difficulties communicating in English and Standard Swahili. However, he said they disguised these communicative challenges as markers of cultural sophistication such as "modernity." He described these students as follows:

This is a generation of the 90s upwards; our current students, yah. This is the dotcom generation. That one also has issues. For them, I think the issue is that they have borrowed a lot or they have leaned a lot on the perception that Kiswahili is for the uncivilized, for the aged. But funny enough is also that, ah, they not only break Kiswahili but also English and they want to say, they want to claim that that is modernity. You are more civilized when you break language rules and this to me is escapism. That someone has no command of a language and he will want to hide in modernity or civilization such that they can communicate by breaking rules so that you do not hold them on anything. Yah, because they cannot communicate consistently in English neither can they communicate consistently in Kiswahili. So they hang up katikati ['in between'], and they say, you know, that this is a dot-com generation. (Mr. Seneta 03/2012, interview) ${ }^{60}$

Mr. Seneta's sentiments indicate that teachers and students have conflicting language ideology and operate in opposing "linguistic markets" (Bourdieu 1977). On the one hand, teachers disparage these nonstandard language practices as "errors," hence markers of communicative inadequacy (Alim 2004; Labov 1972; Pullum 1999). On the other hand, students regard them as indices of "modernity", "civilization" or

\footnotetext{
${ }^{60}$ In my own observations, the term "dot-com generation" was introduced in Kenya in the late 1990s and early 2000s to refer to the younger generation, which had enthusiastically embraced the internet and social media. Actually, young people then thought highly of having an e-mail account as a marker of "modernity."
} 
"currency" for negotiating a sense of belonging to a different generation. However, this observation should not be misconstrued to mean that all teachers are opposed to nonstandard language varieties. Nor are all students opposed to the officially sanctioned Standard Swahili and English. Instead, some teachers and students belong to multiple communities of practice, sometimes with competing values and practices. As a result, it is common to find same teachers or students expressing conflicting attitudes in various contexts. For example, Mr. Fujo, the teacher of Standard Swahili from Mombasa, criticized Sheng and lugha ya mtaa arguing that they affected students' mastery of Standard Swahili. However, he reported that he sometimes spoke Sheng in order to "fit in," especially when in the company of friends. Mr. Fujo's contextual language attitudes toward Sheng are captured well in the following extract:

MW: And as a teacher of Swahili, how do you perceive Sheng in the context/in relation to the Swahili that you teach?

Mr. Fujo: In the context of the Swahili that we teach, it is distractive. Distractive in the sense that, ah, people are very careless. No one cares about the grammatical mistakes that you make because, in fact in Sheng the mistakes are part of the language. Actually, in Sheng there are no grammatical mistakes. No grammatical mistakes at all. Whatever you speak is right. No one can question. After all, that is my Sheng, you have yours. So, everyone is independent. I think that independence attribute of Sheng is what makes it to flourish more as opposed to the language where somebody has to tell you that this is right or this is wrong.

\section{MW: And what about other teachers of Swahili. How are they talking about it?}

Mr. Fujo: Definitely no one is happy about the Sheng issue. As much as I also do speak Sheng, I do speak Sheng, but, eh, in the context of a classroom, I will never speak Sheng.

\section{MW: What are some of the contexts where you speak Sheng?}

Mr. Fujo: Of course when am with my friends. For example, today if I travel to Nairobi, I will speak my Swahili for a few minutes then I will find myself switching to Sheng to fit in that context because we cannot speak two different languages. They either speak my Swahili or I speak Sheng. (Mr. Fujo 01/2012, interview)

In the case of students, recall that those in leadership positions such as

Mwelusi in Nakuru and Myumba in Mombasa had to negotiate a balance between 
pleasing the school administration on the one hand, and associating with peers on the other. The same was true with good academic performers. In this regard, their attitudes were usually contextual and sometimes contradictory. (I will discuss students' paradoxical associations to the dominant official ideology in Chapters Six and Seven.)

Owing to the earlier claim by Mr. Seneta, that there was a disconnect between language use patterns in the school and in the wider society, I asked him how parents responded to their children's use of unauthorized language varieties such as Sheng.

He claimed that there were three categories of parents as follows:

Mr. Seneta: Aah, we have different types of parents. We have those whom their children rule over them, and also even speak Sheng just to adapt to their children. Yes, we have those who speak Sheng because their children rule over them; children dictate. Then we have those, like me, who are strict; that is, if you want to talk to me in Kiswahili, then speak standard Kiswahili. When you are talking Kiswahili or English, then speak Kiswahili fluently and standard.

MW: Hakuna mambo ya Sheng? ['So you don't approve the use of Sheng?']

Mr. Seneta: No, let's not have shortcuts. Those shortcuts are very long cuts. In fact, they are not shortcuts. Shortcuts are never always shortcuts. They are very long cuts. Yah, but ok any language, if I am speaking in my vernacular, we can talk, the longest we have talked, but I am not going to trail over English

MW: Wewe unataka mtu ashikilie lugha moja? ['Do you want a person to stick with only one language?']

Mr. Seneta: Shikilia lugha moja tuchape bwana. Eeh tumalize bwana. Kama ni Kiswahili, tuzungumze Kiswahili. Kama ni Kingereza, tuzungumze Kingereza. Kama ni kuchanganya, ['Keep to one language, let's stick man. Eh let's stop. If it's Swahili, let's speak Swahili. If it's English, let's speak English. If it's mixing,'] then we must respect the standard version of what we are mixing.

MW: Mmh, so you are ok with mixing standard elements from many languages?

Mr. Seneta: Yes, I can mix but depending on the context also. In official contexts, of course that is not allowed. But in private talks, I can transgress and utter one or two words here and there. But si mambo haya ambayo hayaeleweki ['But not this stuff that can't be understood']. (Mr. Seneta 03/2012, Interview)

Mr. Seneta's response reveals further effects of the "standard language ideology," but I will discuss them one at a time, drawing comparative links 
from Nakuru and Mombasa. His first claim that parents who spoke Sheng to their children had lost parental authority is closely related to that made by $\mathrm{Mr}$. Nyachae earlier that students' pervasive use of Swahili and Sheng was an indication that the school administration had lost its authority over students. Mr. Nyachae was also a staunch adherent of the "standard language ideology." He swore that if he became the school Principal, he would be very strict on the language policy. He said he would display the school rules in various places within the school for the students to read. Also, he would counsel them on the need to speak the authorized languages, including that they would not answer the examinations in Sheng. According to Mr. Nyachae:

If the school enforces those rules, we will have it alright because, if you are passing around the corridors and you hear students speaking in Sheng, it means the school has no policy. But if for example, lemme tell you, if I became a Principal of this school, the first thing I will ban is Sheng. (Mr. Nyachae 11/2011, Interview)

Mr. Nyachae's emphasis on the need to have a strict language policy and additional rules to reinforce it evokes Bourdieu's (1977) claim that a "legitimate language" has to be protected and sustained by a permanent effort of correction.

The other sentiment by Mr. Seneta that students should not mix languages in their conversations but instead stick to the standard is informed by what Siegel (2008) refers to as "interference concern." The two terms refer to the fear common among educators and the general public that use of the unauthorized language interferes with the mastery of the officially sanctioned one(s). Mr. Seneta's strong emphasis that speakers should stick to the standard even when mixing various languages in a conversation, rather than using "stuff like Sheng which cannot be understood," points to another concern 
associated with the "standard language ideology," that people write the way they speak. In Hawaii, for example, Siegel (2008: 59) reports how the Chairman of the Hawaii State Board of Education opposed the use of Pidgin in schools, arguing that, "[i]f your thinking is not in Standard English, it's hard for you to write in Standard English. If you speak Pidgin, you think in Pidgin, you write in Pidgin... We ought to have classrooms where Standard English is the norm.",61

Mr. Seneta's reference to Sheng as a "shortcut" and his caution that "[t]hose shortcuts are very long cuts" suggest a belief that nonstandard language varieties such as Sheng compromise the communicative efficiency of the speaker. ${ }^{62}$ I recorded similar sentiments from some teachers in Nakuru, who alleged that students who spoke Sheng had problems in using "proper language." For example, Mrs. Padri, a teacher of English stated that:

Students in my previous school would resort to Sheng because they may be handicapped or something. Because they find it very difficult, most of them, to express themselves in English, which is the official language of communication in the school, as per the school policy. But here, they choose what language to use and sometimes you may find them using English among themselves but mostly Kiswahili. So, if one were required to use English, they would not have any problems but students in the other school would, given a chance, use Kiswahili throughout even during an English lesson. (Mrs. Padri 10/2011, interview)

In addition to communicative inadequacy, people who subscribe to the "standard language ideology" also associate nonstandard language varieties

\footnotetext{
${ }^{61}$ Different people use different names when referring to the Hawai'ian Pidgin. Some people call it just Pidgin, others Hawai'ian Pidgin and the rest Hawai'ian Creole. Some scholars like Siegel (2008) prefer calling it Hawai'ian Creole, arguing that the label Pidgin demeans its status because it has already gained native speakers among other reasons. In this chapter, however, I employ these labels as used by the particular individuals or groups that I quote.

${ }^{62}$ While school authorities' reference to nonstandard language varieties as shortcuts suggests communicative inadequacy, speakers' use of the same labels suggests communicative sophistication. This is because communicative competence in the two social categories is evaluated differently; while school authorities rely on the use of the standard, speakers of nonstandard language varieties rely on the ability to draw from multiple languages and ingeniously manipulate the form and meanings of the borrowed materials.
} 
such as Sheng with stigmatized social groups such as Choka Mbaya, Marui or Manamba. They also associate these language varieties with low-income or crime-prone areas commonly known as slums. Conversely, they associate English with the elite and affluent neighborhoods. Mrs. Padri, for example, noted:

From my own observation, most of the students in our school come from the elite families but students in my former school come from the slums; slum area, those low income estates. Yah, so students in this school maybe came from some good primary schools, some of them may have come from academies meaning that their parents are elite. So, they could afford to take them to good primary schools. That is where they got the background because in private schools, right from nursery school, the kids speak in English and even good Kiswahili. You don't find so much of the Sheng being spoken. (Mrs. Padri 10/2011, interview)

While it was true that there were students in the Nakuru School who came from the affluent neighborhoods, the majority of those that I talked to reported that they were from the low income areas and they spoke Sheng. It is probable that Mrs. Padri's sentiment was informed by the common romanticization of the link between speaking "good" English, high socioeconomic status and academic excellence, which is not always true. In fact, some sociolinguists have disputed this claim, which was common at one time among educational psychologists. In the U.S, for example, Labov (1972) argues that what makes the speech of the middle-class English speaking children more striking to the listener than that of lower-class speakers of Black English Vernacular (BEV, now usually called AAVE), is the verbosity of the former rather than their greater logic or intelligence. Labov defines verbosity as being wordy, redundant and explicit when speaking or writing. That is, a speaker "makes every effort to qualify his/her opinions and seems anxious to avoid any misstatements or overstatements" (Labov 1972: 218). According to Labov 
(1972), what educators should know is that the two categories of speakers use different speech styles, which are generated from different cultural contexts. In this regard, he charges that what differentiates the two speech styles in educational and other formal settings is a matter of social acceptance, rather than one variety being more logical than the other.

In the case of my research, some teachers and students in Nakuru who discovered that I was interested in Sheng referred me to specific schools. They told me that students in those schools spoke Sheng "properly," that is, in a manner that was intense and unintelligible. Mr. Nyachae for instance said:

I think you have gone to the field there and you can hear them speaking in Sheng. But this school is not even, eeh, this one is not. Here, Sheng is not properly spoken. You go to schools like, eeh, which have Rugby, which is really entrenched and hear those boys. Whatever they talk; it's all Sheng... There is a school, though not in Nakuru here, and that was a private school. Now, if you want, eeh, Sheng proper, go to these private schools which are again not performing very well. And mixed especially. Aah, you can get Sheng properly. And if you want to do research, go to those ones. (Mr. Nyachae 11/2011, interview)

I have noted further above that several other teachers in Nakuru also claimed that schools where students spoke Sheng had discipline problems. However, Mr. Nyachae was the most vocal of them all, possibly because he had headed various schools in different parts of Kenya. He made a contrast between two high schools, one that was private, mixed-gender and performed poorly academically and another that was a prestigious, male only, and an academic "powerhouse." He described the two schools as follows:

In Ukavini, there is a school that was called, eeh, what was the name? This school, eeh, was a mixed school, eeh, but you see the students there, they speak Sheng totally and even the behavior, it really made even teachers from other schools to report to the DEO [District Education Officer], and the school was banned from games because of the way they were behaving. It was a private school. It was an Indian, ya Mhindi ['Swahili for Indian']. You see, the behavior, you know if there is no discipline, let me tell you, in a school where there is proper discipline, even Sheng you cannot hear it. You can't. May be you speak it out there. Now, lemme ask you: You go to Ustaarabu Boys, I am sure, if you find somebody speaking Sheng, even he 
might not stay. You will dig out and find that this is indiscipline, yah. (Mr. Nyachae $11 / 2011$, interview)

Mr. Nyachae's sentiments above project him as one of those teachers who are strongly opposed to students' use of nonstandard language varieties such as Sheng. However, some of his colleagues, especially in guidance and counseling, who had also strongly advocated for students to speak English or Standard Swahili, later confessed that they sometimes allowed clients who could not adequately express themselves in the two official languages to use Sheng. I therefore sought to know whether there were some contexts where Mr. Nyachae would evaluate Sheng positively. I asked him to comment on whether Sheng had a positive role in academic settings. He emphatically responded:

To me, it doesn't because when I am counseling a student, I am counseling him to be an all-round student: in terms of morals, in terms of language, in terms of behavior, in terms of everything. Isn't it? So, am I going to counsel him, encourage him to speak in Sheng instead of discouraging him? (Mr. Nyachae 11/2011, interview)

Mr. Nyachae was also opposed to students' use of ethnic languages and described speaking these languages in very demeaning terms such as "shouting" in Kikuyu or Dholuo rather than "speaking" the two languages. He argued that students should only speak English and Standard Swahili. He also added that ethnic languages should not be used in public spaces such as government offices, because of their exclusive nature. His sentiments were very emphatic and authoritative:

I am saying only two languages! Because I will not again want to see students competing here in terms of which language becomes better. I come from Kisii, I am now talking in Kisii, my Kisii is there. Others are shouting in Kikuyu there, others in Luo there. Now, that becomes, No! The school should have uniformity. And uniformity means, if there is a rule to be followed, it should be followed. (Mr. Nyachae 11/2011, interview)

Mr. Nyachae's call for uniformity within the school invokes Bourdieu's (1977: 652) argument that in multilingual situations, "in order for one form of speech among 
others to impose itself as the only legitimate one, in short, in order for there to be a recognized domination, the linguistic market has to be unified and the different class or regional dialects have to be measured practically against the legitimate language." But is the said uniformity limited to the use of the officially sanctioned languages, or does it cut across other aspects of students' behavior such as manner of dressing? I engage this question here below.

\subsubsection{Language vs. Body hexis: the dialectics of uniformity in Kenyan schools}

According to Bourdieu (1977: 660), language is only "one dimension of the body hexis in which one's whole relation to the social world is expressed." $\mathrm{He}$ defines body hexis as a durable way of presenting one's whole self, i.e., the body, with regard to ways of speaking, thinking, walking and even standing, for example, when addressing or interacting with figures of authority in order to earn social acceptance or approval. Body hexis is learned early in an individual's socialization process, thus it is usually hard to control or change it voluntarily. It is for this reason that Bourdieu argues that what speaks is the whole social person.

School as an agent of socialization prescribes certain body hexis to the students, including dress code and other socially acceptable ways of holding their bodies when talking to teachers or sharing space with other school authorities and visitors. For example, each of the two schools where I conducted my research had a student code of conduct. Some of the rules were printed on a piece of paper and provided to the students at the time of admission while others were disseminated verbally during school assemblies. Each student was required to append his/her signature that s/he had read, understood and would abide by these rules for the entire 
period of study. Failure to abide by the rules could lead to sanctions or expulsion from the school. Vikapuni School in Mombasa had the following rules directly related to Bourdieu's (1977) notion of body hexis:

(i). Students should not pocket/stand akimbo while talking to their teachers/visitors in the school compound.

(ii). Boys shall be expected to be on shirts with proper stitched badges, a grey trouser, black school shoes and white socks.

(iii). Girls shall be expected to be on white blouse with properly stitched badges, a grey skirt, white socks, and black school shoes.

(iv). No alteration, deformation etc., is allowed on the school uniforms. They must have buttons at all times and whenever out should be replaced immediately.

(v). Members of staff and visitors should leave the class first. After this, then any female students wishing to leave and lastly the males.

(vi). Bizarre hair-dos outside limits of reasonable style are not allowed. Straight perm is allowed, no curly kit and Rasta's.

Someni School in Nakuru also had rules related to body hexis. Some of the rules addressed the expected student behavior when outside the school. They included:

(i). All students will wear school uniform at all times, no jewelry is allowed.

(ii). Full uniform will be worn when a party goes on a visit outside the school.

(iii). Girls are not allowed to put chemicals on their hair. We only allow neatly plaited push back lines.

(iv). Students will behave in a proper and respectful manner at all times. They will not do anything which breaks the laws of Kenya.

(v). P.E kit will be worn for P.E.

(vi). Students must obey without question all teachers, prefects, monitors, and all other persons in authority.

A critical look at the various school rules both in Nakuru and Mombasa reveals that the prescribed body hexis produces and reproduces the power differentials between teachers and students. For example, both schools had a defined vertical flow of command between students, teachers and the school Principal. In addition, the Nakuru School did not allow students to question authority at all. Some teachers had internalized this social order so much that they did not recognize students' agency in shaping their own lives such as when making language choices. Instead, teachers characterized students as "lacking," and perceived them as "vulnerable" and passive 
social agents who lacked full control of their lives. As a result, teachers believed that students needed to be monitored and corrected always. For example, I asked Mr. Nyachae to comment on why students in his school spoke Sheng most of the time rather than English and Standard Swahili. He responded as follows:

I have told you. It is just indiscipline and peer influence. You see, and that is why I have told you, in my class, they cannot speak Sheng but when they are around there, they can. They are influenced. Then, if we are to say here, there is no speaking Sheng. That is the cardinal rule. One of the rules, rule number this [gesturing], the students will. And you know that is why I have told you: the schools which perform, the student is there to toe the line. Follow the school rules. Simple! And then things will work. (Mr. Nyachae 11/2011, interview)

Several other teachers in Nakuru and Mombasa attributed students' use of Sheng and lugha ya mtaa to lack of a "reading culture." For example, Mrs. Kiboko, the teacher incharge of guidance and counseling introduced in Chapter Two, claimed that students spoke Sheng most of the time, and this affected their ability to use Standard Swahili and English, especially when they came to her office for guidance. When I asked her to elaborate, she not only blamed Sheng but alleged that students lacked a reading culture too. These sentiments are captured in the following extract:

Mrs. Kiboko: So sometimes you get answers or words and expressions that just lose you because the direct translation from what they have thought in their mind now they are putting it into the word they think you will understand. It all comes out crazy.

\section{MW: And is that language deficiency attributed to their use of Sheng?}

Mrs. Kiboko: Oh yes, it is because another thing about our children they also don't read.

MW: Ok, ooh ooh, I have heard several teachers talk about lack of a reading culture

Mrs. Kiboko: Reading culture is not there and I have really been trying to inculcate this in them that, you know when you read, you grow your language to a point where if I ask you to describe something you don't have to experience it to describe, ok? But because you've been reading, you can visualize. You know it also grows your ability to imagine and you are able to put down something really extraordinary, you know? Recently there was this competition and children were writing these essays.

MW: Oh yes I saw it outside your office about... 
Mrs. Kiboko: And one girl wrote an essay and the Principal, looked at it and said, 'my, my can I have a dictionary?' One of the words there, he said, 'I have never heard this one.' And you know he passed on the essay to me and I read it and when I read, I found out that this child had really written brilliant. And we even had to call this girl and find out where did you [she] go to school and all that. She is an academy child, and the language was marvelous, you know? Though, when we called her, she panicked a bit because now she didn't know why we were calling her. And you know when you are called to the office when the deputy is there, the Principal is there, you might think that you have probably done wrong. So, she got tongue-tied a little and she couldn't talk. But after that, I got to talk to her and reassure and told her, 'you know we were just amazed at what you had written and wanted to see the face behind this essay and that was beautiful.' You know? She wrote well, yah.

(Mrs. Kiboko 11/2011, interview)

While it is true that a reading culture may help improve speaker's knowledge in a language, I however, realized later that it was not that the students did not have a "reading culture." Also, neither was use of nonstandard language varieties the problem as these teachers purported. Instead, it is my conclusion that there was a conflict in ideology between the teachers' generation and that of their students about "legitimate" knowledge and its sources. For example, Mrs. Mob from Nakuru alleged that many students rarely read the books from the school library since they found them "too old" for their generation (Mrs. Mob 10/2011, interview). She said that it was only the bright ones who read such books. The rest preferred reading materials that were prohibited by the school authorities. These ideological differences are reflected in the following extract:

MW: I remember in one of our conversations you said that the reading culture among students and, youth in general, is dying, and we are in the library and I can see it's full of books. What did you mean?

Mrs. Mob: But the students will not value these ones. These ones are too old for them

MW: [laughs] What kinds of books do they read, if they do?

Mrs. Mob: Them, they read the novels. Those ones that have, what do I say?

MW: Mmh, [laughs]

Mrs. Mob: [laughs] 


\section{MW: Oh yah content?}

Mrs. Mob: The content is far beyond the language ya classroom ['classroom language']. Social life. It is about social life. That is what I would say. Otherwise, they cannot come and pick such a biology book and start going through. Maybe the very bright ones.

\section{MW: And in what language or languages are these novels published?}

Mrs. Mob: Some of them are in Kiswahili, others are in English and for the ones who love French, they will go for the French novels.

MW: Oooh

Mrs. Mob: Eeh

MW: And why novels? Why not any other kind of book?

Mrs. Mob: Me I cannot tell unless you answer that for me because you are youth.

Both: [Laugh]

MW: But you see I am a Swahili teacher?

Mrs. Mob: [laugh] Unless you tell me what usually makes them...

\section{MW: Read novels?}

Mrs. Mob: Read novels, read, read, read magazines that are out of place. Me I buy a lot of magazines. In my house they are so many, but even my sons and daughters will not go for those magazines.

(Mrs. Mob 10/2011, interview)

In the extract above, Mrs. Mob said that students preferred reading materials about social life such as magazines and novels whose "content is far beyond the language ya classroom”, 'language of the classroom'. They also preferred watching movies and visiting cyber cafes to surf the internet. Mrs. Mob's sentiments point to the generational difference between teachers and students, in that while her generation takes pride in reading academic materials and storybooks to improve one's command of the officially sanctioned languages, the students' generation takes pride in the number of movies that an individual has watched, and how up-to-date s/he is with the current forms of technology. 
The sentiments expressed by the various teachers above about speakers of nonstandard language varieties such as Sheng and lugha ya mtaa conform to what other figures of authority in the wider Kenyan society believe about Kenyan youth in general. According to Murunga and Nasong'o (2007: 129,159):

While youth in Kenya refers to that transitory period to a future in which they can begin to participate in society...youth as a social category has traditionally been viewed as a burdensome category that needs to be 'carefully handled' and whose energies need to be channeled to 'productive' endeavors ostensibly by elders and more 'responsible' members of society.

In this regard, some teachers believe that tightening the school rules is the only means to ensure that students conform to prescribed behavior. For example, Mr. Nyachae observed that such strictness had been successful with regard to the dress code in his school. He said:

And lemme tell you, this school has a culture of uniform. Have you seen the Relini students' uniform? I think it is the only school where you will find, it is very hot but they are in full school uniform. Why? The rule. And they adhere to it. (Mr. Nyachae $11 / 2011$, interview)

As a class teacher, Mr. Nyachae reported that he did not tolerate students who modified their school uniform or engaged in "sagging," the term used for wearing pants below the waist. He said that he usually told students who "sagged" to adjust their pants instantly, and punished those who continued doing so. I recorded similar sentiments from teachers in Mombasa. Mrs. Munene, for example, reported that her school strictly enforced the prescribed student dress code for various reasons. From within the school, she said that school uniform enhanced homogeneity and equality among students. It helped them transcend socioeconomic differences that would otherwise be conspicuous if they were allowed to wear home clothes. In addition, she said that school uniform made all students look the "same," hence "neat," "smart" and “decent." The prescribed "decency" also applied to haircuts, shoes and so on. 
The issue of "decency" is important to my dissertation project because my field observations indicate that enthusiasts of Sheng and lugha ya mtaa differed with school authorities about how a "decent" person looks. While school authorities defined "decency" in terms of uniformity with regard to language and related behaviors including manner of dressing, many students defined it in terms of "difference." This conflict is well captured in the following statement that I recorded from a student called Mvunjez in a focus group in Nakuru. He said that, "like for me if I come here with a bling bling, to the Principal it is indecent" (Praise and Worship focus group, 11/2011--Nakuru). (See next chapter for further discussion on decency.)

Going by Mrs. Munene's sentiments, I argue that the school uniform was a badge of identity that distinguished their school from the neighboring ones. For example, she told me that there was a time certain schools known for discipline problems had started "copying" her school's uniform. In one of those schools, she said that girls used to modify their skirts, making them "so short and very tight." To her school's disadvantage, she claimed girls from these other schools would be confused by the wider society with girls from her school. Mrs. Munene claimed that the confusion could have severely tarnished the reputation of her school because of the social values that it upheld. As a result, her school had to switch to a different uniform to enhance distinctiveness and protect their identity. She elaborates: "you know we have a name to protect: we are proud to be a Christian, missionary school and there is that image that we want to put outside there. You see, yah. So, we decided that we just get rid of this uniform and have something unique" (Mrs. Munene 04/2012, interview). In order to enhance the desired uniformity, Mrs. Munene said that her school contracted with a particular clothes designer. The 
designer came to school when needed to take measurements for students' uniforms. When ready, the designer delivered the clothes to the school Principal for distribution to individual students in order to protect the integrity of the uniform code.

Mrs. Munene added that teachers conducted random checks to catch students who altered or modified their uniforms, by such tactics as removing the band at the hem of the skirt. When they found such modified uniforms, they usually confiscated and donated them to a neighboring orphanage, then asked the affected students to buy new ones. The same strictness applied to other prescribed aspects of "decency" such as hair length. According to Mrs. Munene:

For the boys, we tell them to have very short hair. Mmh, just very short. That, aah, you know sometimes, they are so careless; they don't want to comb. So, when they keep cutting them short, then it doesn't look really bad. And, for the girls, you just make, aah, you make lines backwards or you make lines up. We call it Kilimanjaro. Or just comb backwards and put a clip of either blue or white. (Mrs. Munene 04/2012, interview)

In summary, the foregoing discussion has traced the extent to which the notion of "standard language ideology" and the related idea of "legitimate language" apply to the linguistic situation in Kenya. Despite contextual differences between the teaching of English in schools in the West. I demonstrated that some aspects of the "standard language ideology" and "legitimate language" apply to the case of English in Kenya. However, both notions do not fit well in the case of Standard Swahili. As a result, English is socially constructed as "superior" to Standard Swahili, despite the fact that both languages are official. In this regard, many Kenyans attach more value to English than Standard Swahili. Therefore, they are usually more concerned about speaking the former "correctly" more than the "latter." The situation is 
even of great significance in Mombasa where Swahili is spoken natively. For example, Vikapuni school had a language policy that required students to speak English all the time, with some teachers arguing that it was an effective way of controlling "noise" and preventing students from "vulgar" discourse, which would otherwise happen if they were allowed to speak other languages including Swahili. The school also employed different measures to reinforce the use of English while discouraging other languages including Standard Swahili.

In Nakuru, some teachers like Mr. Nyachae alleged that students spoke Swahili and Sheng most of the time because the school did not have a language policy. They interpreted this linguistic situation as an indicator that the school administration had lost control and authority over the students. These teachers also regarded parents who allowed their children to speak Sheng as having lost parental authority. The teachers were biased toward students who spoke English. In some cases, these teachers romanticized speaking "good" English and associated it with being "courteous" and "intelligent." On the other hand, they associated nonstandard language varieties such as Sheng and lugha ya mtaa with students or schools that performed poorly academically or had discipline problems. They alleged that these were the schools where Sheng was spoken "properly," and even advised that anybody seeking to study Sheng should focus on such schools. However, as I mentioned earlier, I found that students in academically successful schools such as Someni and Vikapuni also spoke Sheng.

Further, the discussion established that the prescribed "correctness" in schools is not limited to language, but also applied to other aspects of body hexis such as 
manner of dressing. However, I found that not all teachers and students fully subscribed to the "standard language ideology." Instead, some associated and dissociated with the officially sanctioned languages and behaviors depending on the context, hence their language attitudes were usually contextual. Those who fully subscribed to the dominant standard/official language ideology claimed that the only way to ensure that students embraced the officially sanctioned languages was to ban use of nonstandard language varieties in schools. Next, I focus on what happens when schools employ such strict measures against nonstandard language varieties and associated mannerisms.

\subsubsection{Does fighting the "usual suspects" eradicate them really? The question of nonstandard language varieties in schools}

The existing literature on nonstandard language varieties indicates that attempts in schools to eradicate these language varieties have generally yielded negative results. However, teachers who build on students' home language and other aspects of students' cultural background often realize positive results (Da Pidgin Coup 2008; Rickford 1999; Rynkofs 2008; Siegel 1999, 2008). In Hawai'i, for example, various figures of authority such as teachers and officials from the Board of Education have always fought Hawai'ian Pidgin, arguing that it affects students' performance in the national standardized tests. Earlier, I alluded to the claim by the Chairman of the Hawai' ian Board of Education that speakers of Hawai'ian Pidgin would not be able to learn Standard English since people write the way they speak or think. Da Pidgin Coup (2008) disputes this claim, arguing that, while it is important for students to learn Standard English, there is no evidence that speakers of Hawai'ian 
Pidgin are less capable of learning to write, or that Pidgin cannot be utilized to facilitate learning. ${ }^{63}$ As mentioned earlier, such negative attitudes toward nonstandard language varieties and their speakers are usually informed by the "standard language ideology," which is prevalent in dominant institutions such as schools and mainstream media.

In the case of Hawai'ian Pidgin, various scholars report that the negative attitudes it faces today gained sanction and approval in the early 1920s, when these attitudes were printed in curriculum materials designed for teachers in public schools and educational journals (Da Pidgin Coup 2008; Siegel 2008). According to Da Pidgin Coup, not everybody evaluated the language negatively. Anthropologists and sociologists, for instance, acknowledged that it was a badge of cultural identity for its speakers. However, Da Pidgin Coup singles out the professors of speech and Standard English at the University of Hawaii, arguing that they are the genesis of the negative attitudes expressed towards the language today. In this context, Da Pidgin Coup (ibid: 32) charges that, " $[t]$ he ears of these professors were trained to hear Standard English; they heard Pidgin not as a different language variety but as English that came short. They used the term lazy language and the adjectives ungrammatical, faulty, sloppy and slothful." Since such figures of authority occupy very influential positions in the society, their language attitudes are often unquestioningly embraced by other people including speakers of the affected languages.

According to Siegel (2008), teachers and school administrators perceive Hawai'ian Pidgin as a deviant form of English and talk about the language and its

\footnotetext{
${ }^{63}$ Da Pidgin Coup is a group of faculty and students in the Department of Second Language Studies (SLS) at the University of Hawaii, who wrote a position paper in 1999 responding to this claim by the Chairman of the Hawai'ian Board of Education.
} 
speakers pejoratively. On the one hand, Siegel reports that these figures of authority often refer to the Pidgin using terms such as "broken", "bad" or "incorrect" English. On the other hand, "they consider Pidgin-speaking children not as learners of a different language but as careless or lazy speakers of Standard English" (Siegel 2008: 56). Da Pidgin Coup (2008: 32) cites two specific examples from the Hawaii Educational Review, showing the kind of attitudes that teachers and other members of the Hawaii society have towards the Pidgin in relation to Standard English. The two examples also demonstrate the active role that dominant institutions such as schools and mainstream media play in spreading the "standard language ideology." The first set of views is from an anonymous author, who says that:

Hawaii is the land of broken English. Tell children that the Pidgin English which they speak is not good English; that it is not spoken by good Americans. Show the children that Pidgin English implies a sense of inferiority. (The New Course of Study 1921: 9-10)

A critical look at these sentiments reveals that language attitudes are not just about language. Instead, they spill over to other aspects of speakers' identity such as citizenship. In this particular case, the anonymous author not only redefines Hawaii as the "Land of Broken English" but also redefines American citizenship in ways that exclude speakers of Hawai' ian Pidgin. This is captured in his claim that, speakers of Hawai'ian Pidgin should be told that they are not "good" Americans since they do not speak "good" English. Owing to the fact that being American has high symbolic capital both in the United States and the rest of the world, such sentiments would make speakers of Hawai' ian Pidign not only feel excluded from the notion of “Americanness," but also inferior to Americans who speak Standard English. Such sentiments indicate that the anonymous author subscribes to the notion of homogeneism mentioned in the previous chapter, which socially constructs linguistic 
diversity as a threat to national unity and therefore seeks to exclude those who do not adhere to the prescribed homogeneity (Blommaert and Verschueren 1998).

Da Pidgin Coup (2008: 32) also quotes an elementary school teacher who says that children should be taught contrasting images to associate Pidgin and good speech. That is:
Words spoken correctly and pleasingly pronounced are jewels, but grammatical errors and Pidgin are ugly. Teachers should tell children that Pidgin was like the frogs, toads, and snakes in the fairy tales they were reading. Good speech was like the roses, pearls, and diamonds that dropped from the lips of the good sister who helped people and was beautiful. (The New Course of Study 1921: 9-10)

Da Pidgin Coup (2008) disputes these views, arguing that they are not only unjustified and biased but also wrong. Instead, both Pidgin and Standard English are equal from a linguistic point of view. That is, "both are fully grammatical systems that their speakers can use for effective communication on any topic and in any situation" (Da Pidgin Coup 2008: 33). However, what makes Standard English seem "superior" to other language varieties in Hawaii are the social and political advantages it enjoys such as the official status as a media of classroom instruction.

Despite these sociopolitical differences between the standard and other language varieties, Siegel (2008) and Da Pidgin Coup (2008) observe that speakers of Hawai'ian Pidgin regard it highly, because it is an integral part of their cultural and local identity. It reflects their historical attitudes, e.g., it is non-hierarchical and inclusive despite speakers' differences in ancestral language and history. Also, it distinguishes Hawaii from the rest of the United States and elsewhere, hence its use generates "covert" prestige among speakers. Due to these connections, both Da Pidgin Coup and Siegel argue that teachers should realize that denigrating Hawai' ian Pidgin amounts to denigration of its speakers, who are the majority of the population of 
Hawai'i. In such contexts, various scholars of nonstandard language varieties argue that attempts by school authorities to ban them in favor of the standard are counterproductive. Such efforts neither eradicate the "unwanted" languages nor do they make speakers embrace the officially sanctioned ones (Da Pidgin Coup 2008; Labov 1972; Rickford 1999; Rynkofs 2008; Siegel 1999, 2006). Instead, speakers develop different negative reactions as outlined below.

Some speakers may become resistant such that the more teachers reprimand them, the more they speak their language. Other speakers may withdraw from the learning process by choosing not to speak or participate, which may be brought about by the fear of saying something "wrong," or appearing "stupid" since they are corrected every time they speak. In some other situations, speakers may develop multiple negative attributes. For example, they may exhibit resistance and withdrawal especially if they start to feel that educators are working against them rather than for them. One such speaker is quoted by Da Pidgin Coup (2008: 35) speaking in Hawai'ian Pidgin: "Education is Western based so da guys who teaching it, they come to Hawai'i, they have hard time understanding our people, so instead of working with us, they going work against us and make us look bad." Such reactions often render language a site of struggle between speakers and teachers, eventually creating a counterproductive learning environment.

Further, there are situations where speakers may accept and internalize teachers' negative attitudes that their language is "bad," leading these speakers perceive themselves as "bad” students. This invokes Bourdieu's (1977) argument that schools naturalize social privileges, such that those who "fail" attribute their failure to personal inadequacies rather than to inequalities of social structure. In such cases, 
students avoid speaking their native languages either in certain situations or altogether in favor of the standard.

Their parents also express what Siegel (2008: 59) calls "Ghettoization concern," which refers to the fear that their children might be socially marked or disadvantaged for speaking their stigmatized native languages. For example, Siegel (ibid: 61) cites a letter from the Honolulu Advertiser, claiming that, "[a]ny child today who grows up speaking Pidgin English will never get a good job and never be able to afford a house." Siegel disputes that claim arguing that there are many successful businessmen, politicians and professionals who speak Hawai'ian Pidgin. Instead, he emphasizes that the real concern that parents and teachers have is that, "devoting valuable class time to a Creole ${ }^{64}$ deprives children of the instruction they need to learn Standard English and in turn to get the benefits that speakers of standard varieties have, thus ensuring that they remain disadvantaged" (Siegel 2008: 61).

From a comparative point of view, Siegel reports that speakers of Creole in Australia also oppose its use for classroom instruction, arguing that it is a way of depriving them of the privilege to be instructed in the kind of English used by the white people, hence condemning them to permanent under-class status (see Snow 1990). Similarly, Rickford (1999) reports about the controversy which surrounded the Oakland School Board's resolution in the United States to utilize Ebonics (also referred to as African American English) in schools to help its speakers in the acquisition of Standard English. Rickford says that among those who opposed the proposal were parents of African American children, arguing that using Ebonics in schools would deny

\footnotetext{
${ }^{64}$ Though the terms Creole and Pidgin are used to refer to different forms of language in sociolinguistics, Siegel is using them interchangeably.
} 
their children the privilege of learning "good" English. They also expressed the ghettoization concern, which partly refers to being marked or stigmatized for speaking the unauthorized languages.

In conclusion, the various fears and concerns expressed by authority figures so far, focus on keeping nonstandard language varieties outside the classroom in favor of the standard. However, it is evident that the more school authorities fight them, the more they flourish and the less conducive the environment becomes to speakers of these languages. The discussion has shown that the case of Sheng in Kenya is an excellent example. The language has been in existence since the early 1960 s and ' 70 s, and continues to blossom despite schools' constant efforts to ban its use among students. In the next chapter, I examine Sheng and related language varieties such as lugha ya mtaa, seeking to unravel the various ways in which speakers engage the dominant official language ideology prevalent in schools and the wider Kenyan society. 


\title{
Chapter Six
}

\subsection{Style, Fashion and Identity: Sheng and lugha ya mtaa}

\begin{abstract}
Nobody at all should interfere with our freedom of attitude, our freedom of our own philosophy, yah, and our freedom of our own existence. So, the old people or those who lived in that generation should realize this is Generation TuGpange and should leave it alone. You see now times have changed and our ways of thinking have changed. Now we tend to see things differently. We tend to see decency differently, you see? So, the reason why these styles vary from one student to another is because each one of the students, you know, think differently of what decency is. So, if a teacher tries to dictate how decency is, they [students] think that this must be something outdated... Because when he was a youth then, things were different. And the way of thinking was different and decency was seen differently. ${ }^{65}$ (Form Four focus group 1, 01/2012--Mombasa)
\end{abstract}

Is it resistance, compliance or both? This chapter focuses on the various ways in which speakers of unauthorized Sheng and lugha ya mtaa engage the dominant official language ideology, which is associated with the use of Standard Swahili and English. The chapter also examines the role of gender in speakers' responses to the said official language ideology. As noted in the previous chapter, school authorities regard nonstandard language varieties as "errors," and associated identities as indices of indiscipline, hence they always seek to eradicate them. However, these nonstandard language varieties continue to flourish because speakers regard them highly, and associate them with linguistic ingeniousness and cultural sophistication. My research findings indicate that speakers embrace Sheng and lugha ya mtaa because their use generates more social rewards among peers than the officially sanctioned Standard Swahili and English. In this context, I argue that Sheng and lugha ya mtaa are nonviolent tools of resistance that speakers use to contest the readily available

\footnotetext{
${ }^{65}$ The ' $\mathrm{G}$ ' in TuGpange is equivalent to the self reflexive 'ji' in Standard Swahili. Tujipange in Standard Swahili means 'let us arrange ourselves'. However, my informants in Nakuru and Mombasa told me that when appropriated in Sheng and lugha ya mtaa, the term means 'we should mind our own business'.
} 
languages, because they find them unfit for their communicative interests and negotiating desired social identities.

At the same time, speakers' embrace of these nonstandard language varieties is paradoxical: some of their social practices suggest resistance to the official language ideology and local cultural frameworks on the one hand, while other practices suggests conformity on the other. In this connection, the chapter will address the following questions: 1) in what ways do speakers employ these nonstandard language varieties? 2) What social practices suggest resistance or conformity? Specifically, how do speakers' language-use patterns and other aspects of behavior such as dressing, articulate with prescribed identities? 3) Since Sheng and lugha ya mtaa draw some of their linguistic material from ethnic languages, what role do these materials play in speakers' responses to the dominant official language ideology? 4) Does gender play any role in speakers' resistance or conformity? I engage these questions in the remainder of this chapter, starting with the rebellious nature of Sheng and lugha ya mtaa.

\subsubsection{Sheng and lugha ya mtaa: styling to rebel}

My field observations indicate that many young Kenyans have a great desire for individuality, i.e., distinctiveness from the older generations and independence from parental and societal control. This desire is well captured in the sentiments expressed by Tinga, the student quoted at the beginning of this chapter. The quotation indicates that speakers of Sheng and lugha ya Mtaa on the one hand and school authorities on the other, have conflicting language ideology about what decency is. Speakers find use of the prescribed "uniformity" with regard to language and body 
hexis (Bourdieu 1977) restrictive and outdated or unfashionable. Consequently, they are constantly innovating new styles of distinctiveness, and to keep up with the current fashion. For example, I asked other students in Tinga's focus group to name some of the interests or things that most youths in Kenya desire to have. Several of them responded that youths mainly desired entertainment, fashion and freedom. Tinga elaborated that though the meaning of freedom may vary between social groups, the most commonly sought were: freedom from home confinement, criticism by parents, and the freedom to associate with their generation's way of doing things such as fashion in dressing and language. Other students kept nodding their heads and even repeating what Tinga had said, indicating that they concurred with him (Form Four focus group 1, 01/2012--Mombasa). I recorded similar sentiments from students in Nakuru. But where do young Kenyans get their notions of style and fashion?

Teachers and students in both Nakuru and Mombasa reported that the media, including television, fashion magazines and social sites such as Facebook, play a significant role in socializing speakers of Sheng and lugha ya mtaa. For example, recall from the previous chapter that Mrs. Mob, a teacher from Nakuru, claimed that many students rarely read books from the school library since they found them "too old" for their generation. Instead they preferred materials which were prohibited by the school authorities such as magazines, novels, movies and visiting cyber cafes to surf the Internet. These claims agree with the existing literature. For example, Samper (2002) observes that negotiation of identity among Sheng speakers is rooted in the various multicultural windows such as the media and capital markets, through which globalization and localization of cultural materials take place. Specifically, Samper (2002: 199) says that: 
Cultural studies has shown that popular culture is an important location for the construction of youth identity; and has superseded the educational system as the principal pedagogical site for young people...Popular culture is the arena where identity is negotiated, not the family, the church, the school, or traditional culture.

Similarly, Leitchty (1995), in Wallace and Kovatcheva (1998: 153), claims that these multicultural windows and capitalist consumer markets circulate youth cultural styles to different parts of the world. In this context, Leitchy says that they help to create "an idea of youthfulness originating from Western capitalist countries particularly the USA with its domination of media culture. For young people in other countries, the American model of youth can represent a dream of modernity to which they aspire." Specifically, these youths are inspired by styles and fashion of African Americans, especially rap or hip hop artists. But why is this category of celebrities appealing to the youth?

Despite the fact that black/African American styles such as in music, dance and sports, are received with admiration that is also mixed with deprecation by the dominant, white middle-class in the U.S., youths in many countries outside the U.S find these styles attractive partly because they resonate with the desire for freedom and distinctiveness. According to Kochman (1981: 130), "black style is more selfconscious, more expressive, more expansive, more colorful, more intense, more assertive, more aggressive and more focused on the individual than is the style of the larger society of which blacks are a part." Kochman elaborates that the notion of performance is central to the life of African Americans: whether it is talking, walking or dressing, they aim at demonstrating their "swagger." In addition, many youths find black hip-hop appealing because its content is usually about freedom and rebellion against middle-class norms. In Kenya, Samper (2002: 212-13) observes that: 


\begin{abstract}
Another important reason why young performers are attached to rap music is because of its connection to what Paul Gilroy has called 'diasporic intimacy'. Young Kenyans, like other youth cultures around the world, face similar social realities including poverty, inequality, access to a transnational culture, and social, economic and political disintegration...Local rap, informed by a global black popular culture is yet another way that young Kenyans are forcing open those moments and spaces of freedom that allow the redefinition of the self. Other Kenyan popular music genres (Benga, Soukous, Swahili pop, Taarab) just do not allow for these youthful moments of freedom for several reasons: 1) they are sung in Kiswahili and ethnic languages, which young people associate with the older generation; 2) they are performed by artists who do not self-identify as 'youth'; and 3) the music does not articulate a global perspective, a modern vision.
\end{abstract}

This was the case in my research. For example, regarding the question I alluded to earlier about what Kenyan youths desired, student Tinga's focus group responded as follows:

MW: Today I want us to talk about the interests of the youth. [...]What are some of the things that come into your mind when we talk about the youth?

Mshie: Now it depends with different people. But according to me, when I hear about youth, I see a young person full of entertainment. A person focused in a certain goal in future and a person who is social and a person who is [*audio not clear] in life.

MW: Na Mkinango, ni mambo gani youth wa siku hizi unaona wanapenda sana? ['What about you Mkinango? What things do you think today's youth like most?']

Mkinango: Actually, they like interacting, making boy-girl relationships.

Tinga: The youth of today are usually characterized by total exuberance; they like behaving you know with freedom to the uttermost, with vigor, yah, and doing everything according to their decision. They just want to be let at liberty all the time. Yes, they don't want to be confined at any place.

(Form Four focus group 1, 01/2012--Mombasa)

From the extract above, it is clear that both male and female speakers desire to be free and fashionable. Later in our conversation, these students attributed their ideas of style and fashion to media celebrities from the U.S., especially African American rappers such as Lil Wayne. Even those informants who mentioned local Kenyan popular artists such as Prezzo and Octopizzo referred to those whose styles resemble those of African Americans. The proclivity to associate with such celebrities is driven by the desire to appear distinct, knowledgeable and up-to-date with the current global 
events. The various attributes add to a speaker's swag, which then earns him/her attention from the opposite sex. The following sentiments expressed by student Tinga's focus group encapsulate this phenomenon:

MW: Huku, what are some of the modifications that boys do to the uniform? Let's talk about modifications to the uniform alone.

Tinga: [...] The other one was actually some of the modification that I have seen, everyone would like their biceps you know to be seen

MW: For the boys?

Tinga: Eeh. So, you see the other seam of the sleeve will hold it like that [points to his biceps] Yah? Most boys like folding their arms, you know, it usually helps to make the biceps bulge.

MW: So, why are the boys so obsessed with the biceps?

Tinga: Like I told you, the two opposite sexes normally fight to attract each other. Thus wherever the two exists there is that fighting towards each other; some are verbal, some are nonverbal, some are through actions. Yah?

\section{MW: So biceps call for girls' attention?}

Tinga: It is one of the factors. Among many factors, yah? It contributes. So, when you keep your biceps alert and with your swagger at pose and with your modification, it works. That's another one. Another one is that, aah, trousers, most youth do not want, these days, do not want baggy trousers. They want, aah, you know, a thin, you know, a skinny trousers, yaah. And the belts they like the big, very big buckles

MW: Hizi za dollar sign? ['These buckles with a dollar sign?']

Tinga: Eeh. Aah you know, like now when it comes, you know, they also want to identify with celebs at school.

MW: There we go! Sasa hii ni swag? (I ask pointing at Tinga's transparent shirt pocket where he has a bunch of printed paper cuttings of Kenya shillings 200 currency notes wrapped with a rubber band but with a real 200 note at the topThis is meant to project him as somebody who is rich and who deals with cash. Note that he uses third person plural when explaining in order not to appear like he is bragging in the eyes of peers).

Tinga: Young money. They want to feel like cash money members.

MW: Ooh cash money?

Tinga: Yah.

\section{MW: That is what it is called?}

Tinga: Yah, it is called cash money because they want to identify with this famous singer. It is a group of rappers calling themselves cash money members or young money, yah? 
MW: Ooh I see.

Tinga: So everyone wants to identify with Lil Wayne, Lecross, Bad Man, so

MW: And are those local guys or they are from the West?

Tinga: No, they are international musicians from the U.S. So, everyone would want to identify wearing such thin trousers, big buckled belts and also small shirts for the chest to protrude, you know, and folding your sleeves. (Form Four focus group 1, 01/2012--Mombasa)

A female student in this focus group, Mshie, echoes Tinga's sentiments that fashion is driven by the desire to be distinct and to attract members of the opposite sex. She said that:

Just according to the way Tinga has said, it depends with the person. According to some girls: there are these girls who think that decency is wearing something long but tight and then others will think that decency where you are just being attractive; making people notice that unapita ['you are passing']. And there are some girls who think decency is wearing this suit called "all womans' fashions," gowns, things like big big, baggy baggy things. It all depends with the girls. But with most of the youth of this generation that I am in; most of our decency contains tight things, minis. If it is a top, at least it shows some parts of your body to attract the men just like the way Tinga was saying, yah. (Form Four focus group 1, 01/2012--Mombasa)

Speakers of Sheng and lugha ya mtaa are aware that school authorities do not approve of these styles. For example, student Mkinango from the focus group above says that teachers do not like that "habit of sagging," and girls wearing short skirts to attract boys (Form Four focus group 1, 01/2012--Mombasa). In this context, I argue that the various aspects of style in Sheng and lugha ya mtaa are speakers' deliberate subversive attempts to challenge the dominant official language ideology and local cultural frameworks. I discuss some of them in the following subsections, including how dress code connects to language.

\subsubsection{Speakers' language choices and associated attitudes}

From the point of view of language choice, recall that school authorities emphasize the value of Standard Swahili and English for communication and 
examination purposes, arguing that excelling in the two languages would lead to good career prospects after graduation. Similarly, Mutiga (2013) observes that many parents, especially the older generations, emphasize that it is important for children to embrace ethnic languages because they are the media through which family values, behavior patterns and other cultural norms are disseminated, failing which they would lose their cultural identity.

However, many young Kenyans do not regard these languages and associated identities the same way as their elders. During a focus group in Mombasa for example, I asked students why they spoke Sheng and lugha ya mtaa most of the time rather than English and Standard Swahili. They responded that peers ridiculed those who spoke Standard Swahili as washamba, meaning “old-fashioned." Similarly, peers scorned students who spoke English, calling them "wannabes", "show offs" and "know-it-alls." They wondered why such speakers pretended to speak English yet they were not "whites/Englishmen." In this regard, I argue that speaking English among young Kenyans is perceived as "unKenyan," because peers perceive those who speak English as people denying their local identity. ${ }^{66}$ Also, peers perceive those who speak English as people who project themselves as more important or of a higher social class than the rest. Some of these sentiments are reflected in the following extract:

1. Oleceleb: But on that point that you asked ati mbona tunakataa kuongea English ni juu unajua ukiongea English itakuwa ni kama wasee wengine ama mabeshte zako wataona ni kama unataka kuwa wannabe; unajifanya unajua kizungu sana kwani sisi hatujui? Ati wewe peke yako ndiye unaweza kuongea kizungu. So we just talk to them to fit in $[\ldots]$.
1. Oleceleb: But on that point you asked why we do not like speaking English, it is because peers perceive those who speak English as "wannabes." Why pretend that you know English as if we don't? As if you are the only one who can speak English. So, we just talk to them [speakers of these nonstandard languages] to fit in [...].

\footnotetext{
${ }^{66}$ This term was also used by a student called Hora in a casual conversation in Nakuru.
} 
2. MW: Wannabe, hebu fafanua what wannabe means.

3. Oleceleb: Wannabe ni mtu anataka kuwa kitu yenye si, something that you are not. Wewe na si mzungu mbona unaongea kizungu? As in, unaonyesha watu wengine wewe ndiye unajua sana kuwashinda. So you are trying to be like them yaani. Mimi huona ni kama show off.
2. MW: Would you please explain what wannabe means?

Oleceleb: Wannabe is somebody who pretends to be what s/he is not. Why speak English yet you are not "white/Englishman?" It is as if you are pretending to know more than other people. It is like you are trying to be like them [whites/English men]. I usually regard it as a show off.

(Form Four focus group 3, 03/2012-- Mombasa)

I recorded similar sentiments in Nakuru. For example, in a focus group comprised of Form Four students who had just finished their national exams, I asked participants to comment on the languages that students used when writing Autobook -- a personalized handwritten record ${ }^{67}$ of farewell messages from close friends -- and what they thought about the character of the owner of the Autobook. Members of this focus group told me that students mainly used Sheng because that was the language they spoke most of the time. They elaborated that peers regarded those who used English as people pretending to be wasomi 'scholars' while those using Standard Swahili were regarded as people pretending that they knew the language. They emphatically claimed that those who used Standard Swahili were usually "in trouble" since peers would wait for the Swahili teacher to give back exam papers, then seek to know what these students had scored; the target students would be subjected to scorn if found not to have performed well." So students preferred to associate with Sheng rather than Standard Swahili and English in order to avoid such humiliation. These students' views are captured below:

1. Eduba: Enyewe unajua unaangalianga chenye inaongelewa sana. Wasee wa hii chuo; wasee hubonga Sheng, aah.

2. MW: Sasa Sheng ndio inatumiwa sana?
1. Eduba: For sure, you know you have to consider what language is spoken most of the time. Students in this school speak Sheng, aah.

2. MW: Now, so Sheng is the language used most of the time?

\footnotetext{
${ }^{67}$ This combines properties of an American yearbook with a scrapbook.
} 
3. Eduba: Sheng ndio inatumiwa sana hii chuo.

4. MW: Ama Msud uko na maoni tofauti?

5. Msud: Watu wengine wakikuona unaandika kwa lugha sanifu wanafeel kama yaani unajifanya. Kama unajifanya vile unajua lugha. Saa watu wengi hawapendi kujiidentify na hiyo nini, na hiyo lugha.

6. MW: Ati unasemaje?

7. Mlazoo: Nilikuwa nasema yaani tuseme wanaprefer Sheng kuliko kutumia Kizungu sanifu ama Kiswahili sanifu kwa sababu ukiongea Kizungu sanifu watasema wewe unajifanya msomi sana na kaa wewe ni mwanafunzi, uongee Kiswahili sanifu wewe sasa hapo uko kwa shida. Ukifanya mtihani, hiyo paper yako ya Kiswahili inangonjewa.

8. All: [laugh]

9. MW: Wasee waone umeperform aje?

10. Mlazoo: Eeeh. Sindiyo, sasa ndio uepukane nayo, unajaribu tu uongee tu lugha yenye wanayoongea eeh.
3. Eduba: Sheng is the language spoken most of the time in this school.

4. MW: Or Msud you have different views?

5. Msud: If some people see you writing in standard language, they feel like you are pretending. It is like you are pretending that you know the language. So, many people do not like identifying with that, that language.

6. MW: Hey, what were you saying?

7. Mlazoo: I was saying that, let's say they [students] prefer Sheng instead of using Standard English or Standard Swahili because if you speak Standard English, they will say you are pretending to be a ascholar. And if you are student and you speak Standard Swahili, now you are in trouble. Once you do exam, they [students] will be waiting for your [graded] Swahili paper to see how you performed.

8. All: [Laugh]

9. MW: For comrades to see how you performed?

10. Mlazoo: Eeeh. Yes. Now, in order to avoid that [humiliation], you try to speak/use the language that they speak, eeh.

(Form Four focus group 2, 11/2011--Nakuru)

Such language attitudes discourage students from speaking the officially

sanctioned Standard Swahili and English in favor of the unauthorized Sheng and lugha ya mtaa because the latter earn speakers more recognition and other social rewards among peers. For example, peers praise those who speak Sheng, claiming that they have swag, which is a highly regarded aspect of identity that distinguishes a speaker from the rest. I interpret speakers' preference for these nonstandard language varieties over the standard as rebellion against "standard language ideology," which socially constructs the standard as a "one-size-fits-all" medium of communication. Similar rebellion against the officially sanctioned languages has been reported among speakers of other urban youth vernaculars in Africa. For example, Kiessling and Mous (2004) claim that speakers of these language varieties are actively exploring alternative cultural forms in search of identity that is meaningful to their lives, 
possibly because they are tired of being manipulated and confined within local cultural frameworks. Specifically, Kiessling and Mous (2004: 312) argue that African urban youth negotiate desired social identities by "distancing themselves from the older generations, from the rural population that tends to live a more traditional way of life, and from the upper social classes...." With regard to Sheng, Kiessling and Mous (2004) claim that speakers' embrace of the vernacular signifies their modernity and desire to shape a "youth identity" that does not necessarily associate with criminal life. I now focus on speakers' use of Sheng in academic contexts.

\subsubsection{Sheng and academic performance: is it a resource or an impediment?}

Some speakers dispute the claim common among educators and other figures of authority that use of nonstandard language varieties such as Sheng interferes with students' mastery of the officially sanctioned languages, and their overall academic performance. For example, in an individual interview with a student called Myumba in Mombasa, he asserted that speakers employed Sheng in communicating in-group secrets and that they understood what languages should be used in academic work. According to Myumba, the negative attitudes expressed by educators toward Sheng were false because he himself spoke Sheng, but it did not interfere with his mastery of English or Standard Swahili. Also, he reported that he had a friend in Nairobi who spoke Sheng but scored excellent grades in exams. Therefore, Myumba faulted educators, claiming that they did not understand how and when speakers employed Sheng since they belonged to a different generation (Myumba 03/2012, interview).

Closely related to Myumba's claims above, some speakers who reported that they employed Sheng in academic contexts claimed that they did 
so productively, such as during academic group discussions and peer teaching. I personally observed students in a mathematics lesson in the Nakuru School doing peer teaching in what some speakers would identify as Sheng. I had specifically chosen to observe this class since their mathematics teacher, Mrs. Mob, had told me in a casual conversation that these students used Sheng when discussing class assignments, and during peer teaching even in her presence.

But why do these students prefer Sheng rather than English, the authorized language for teaching mathematics, or even Standard Swahili, the other official language? Recall from Chapter Four that Sheng enthusiasts claimed that Standard Swahili is communicatively limiting and burdensome, because one has to abide by its complex grammar. Also, they claimed that English sounds formal and authoritative, two attributes that are socially unacceptable among peers. Such claims challenge the dominant official language ideology which associates Standard Swahili and English with communicative efficiency, hence academic excellence, while disparaging nonstandard languages as the opposite.

Following Mrs. Mob's claim that her students employed Sheng to learn mathematics in her presence, I sought to find out whether there are teachers who employed Sheng when interacting with students. Some teachers reported using Sheng productively; especially if they realized that some students were having challenges expressing themselves in English or Standard Swahili. Others reported that they employed Sheng when reaching out to young people in general, suggesting that they subscribed to the language ideology that 
associates Sheng with the youth. For instance I noted in Chapter Two that Mrs. Kiboko, despite her high regard for the use of English and Standard

Swahili, sometimes allowed her student clients to use Sheng during guidance.

This allowance was especially extended to those with serious questions or issues but who could not express themselves adequately in either Standard Swahili or English (Mrs. Kiboko 11/2011, interview).

There are also media reports about teachers of English who claim that Sheng is a resource rather than a "problem" in academic contexts. Some confess that their teaching and socialization with students has benefited from their use of Sheng. One such teacher, Ashford Kimani, who teaches in

Nairobi, was quoted in The STAR Newspaper March 13, 2012 saying that:

To blame Sheng on the poor national grade for languages is to make a mountain out of a molehill or small issue. To the contrary, Sheng inflates the learner's resourcefulness due to the exposure that comes with it. As a language teacher of many years' experience, I strongly disagree with this assertion. Writing and speaking are majorly dictated by one's exposure. I have been born and brought up in the rural schools. My degree and intensity of expressiveness has greatly improved since I came to Nairobi city. I can attribute this to Sheng among other factors. In fact, if you asked me mother tongue interference has more disastrous effects on both spoken and written languages than Sheng. I am even worried that KNEC [Kenya National Examinations Council] like some language teachers is simply using Sheng as a scapegoat to justify ineptness. Sheng has bridged the social gap between teachers and students. Consequently both parties understand one another more effectively. Because Sheng is not an instructive language the bonding between the learners and staff is enhanced thereby increasing the level of problem solving and interaction. I know of some national schools that have set aside a day for Sheng speaking. During this day, everybody including the Principal expresses themselves in 'fluent' Sheng. ${ }^{68}$

Despite the claims above that Sheng helps reduce teacher-student social distance bond with students, I observed that there were contexts when speakers employed these language varieties to criticize and challenge authority. I address this question next, drawing specific examples from Nakuru.

\footnotetext{
${ }^{68}$ See this page for more: http://www.the-star.co.ke/news/article-26664/sheng-does-enrich-englishlanguage
} 


\subsubsection{Use of Sheng to criticize and mock authority}

Some speakers employ Sheng to challenge the authorities in schools and in the wider society through parody and mockery. For example, the extract below from a hand-written weekly column called SHAMRA SHAMRA ZA SHULE YETU 'Happenings in our School'was posted on a classroom wall in one of the Grade 12 classes in Nakuru. The column was authored by one of the well-known innovators of Sheng in that class, who was also known for entertaining peers through ridicule and mockery. In fact, other students referred to him by his nickname, Kinaya, 'Irony'. In this particular extract, he mocked the way some teachers including the school Principal behaved, when they participated in street demonstrations in Nakuru town during the national teachers' strike. Those who participated in this strike sought to pressure the government to employ more teachers, arguing that there was a severe shortage of teaching workforce countrywide. The extract reads:

UuuuuW! Finally back 2 school na Mamode wamestrike. Imagine maOdijo! Wanatunga mapsyke songs ati wanariot ka mastude. Kama mode anaeza behave hivo, Sembuse mastude!! Now, watavunja madirisha au watachoma malab? Mr.Diabro na mabranchez akijifanya Abbas akisema ametok'lezea na Boskiey anadai pahali amepitia wengine hawawes make it. Bokono jana alikua tao na B.G wanaunda ma sign boards zimeandikwa "NO MORE MODES NO MORE SKULI." Alafu, Bokono ameng'aria tisho ya msaada imebandikwa "SOLIDARITY 4EVA. ${ }^{, 69}$

UuuuuW! Finally back to school and teachers are on strike.

Imagine teachers! Are composing solidarity songs like students. If a teacher can behave like that, what about students? Now, will they break the windows or will they burn the laboratories? Mr. Diabro holding branches mimicking Abbas saying he has shone and Boskiey claiming no one else can go through where he passed. Yesterday, Bokono was in town with B.G designing placards written "NO MORE TEACHERS, NO MORE SCHOOL."Then, Bokono wore a donated T-shirt on which was written "SOLIDARITY FOR EVER.", 70

\footnotetext{
${ }^{69}$ Students in Nakuru, as noted earlier, appropriated Bokono, a name of a dictator in a Swahili story book, to refer to the school Principal because of his strict and harsh administrative style..

${ }^{70}$ Note that Kinaya writes the word "to" as " 2 " in the first line and "forever" as "4eva" in the last line of the extract. This is one area that I propose for further research later in the dissertation.
} 
Kinaya reported that some teachers were carrying tree branches/twigs while others like their Principal were waving placards on which was written "NO MORE MODES NO MORE SKULI' 'No more teachers no more schooling'. Modes is a Sheng word for teachers, which is unlikely to be used by the school Principal. Probably, it was Kinaya who translated the writings on placards into Sheng, indicating that Sheng fits well in attacking authority. Also, Kinaya assigned teachers demeaning social attributes, attacking their authority and associated identities. For example, he reported that the Principal wore a donated t-shirt. In addition, he wondered how striking students would behave if teachers who are custodians of good behavior conducted themselves so badly.

As discussed earlier, school authorities pejoratively refer to Sheng and lugha ya mtaa as languages of "streets" and "ghetto." Conversely, speakers of these nonstandard language varieties regard them highly. In the following subsection, I address how speakers' high regard for Sheng and lugha ya mtaa articulate with the "standard language ideology" promoted in schools.

\subsection{4. "Streets" and "Ghetto" as sites of ingeniousness}

Speakers' high regard for Sheng and lugha ya mtaa, spoken nonstandard vernaculars associated with the "streets," contravene the "standard language ideology" in various ways. To start with, the two language varieties are neither taught nor promoted in schools nor do they rely on a stable dictionary in evaluating “correctness" or "communicative competence."71 "Standard language ideology"

\footnotetext{
${ }^{71}$ Recall that teacher Ashford Kimani, cited above, said that Sheng was effective for learning because it is not an "instructive language". Possibly, he was referring to the need to monitor one's speech when speaking an official language in order to avoid mistakes. It is also possible that he meant that Sheng is not authoritative. The two possibilities are closely related to students' earlier claims in Chapter Four
} 
privileges written or codified language varieties over spoken ones (Lippi-Green 2012). Therefore, communicative competence in the standard languages is evaluated using written exams and strict adherence to prescribed rules found in dictionaries. It is also evaluated on the basis of the speech and writings of "legitimate" sources such as language teachers and grammarians. Conversely, competence in Sheng, for example, is evaluated based on how trendy and up-to-date a speaker is with the current innovations, which is demonstrated primarily in his/her speech (Samper 2002). Even in written forms of Sheng, competence is evaluated based on the ability to use shortened or manipulated innovations which change from time to time. Thus, failure to use up-to-date Sheng marks the speaker as oldskool: outdated and unfashionable among peers (Githinji 2006; Rudd 2008).

At another level, speakers of Sheng and lugha ya mtaa seek to challenge the official language ideology by focusing on the "streets" as active sites for innovation rather than as sites for crime and other gangster activities. For example, while school authorities' reference to Sheng and lugha ya mtaa as "street languages" is pejorative, Sheng speakers employ "street" as a metaphor for the "site of smartness" (Samper 2002). Similarly, while school authorities stigmatize residential neighborhoods where these nonstandard language varieties are spoken referring to them as "slums" and "ghetto," speakers' use of "ghetto" is positive. For example, recall from the previous chapter that Mrs. Kasisi from Nakuru associated students who spoke Sheng with slums and those who spoke English with affluent neighborhoods. She argued that

that they find Standard Swahili difficult and communicatively inflexible because of its complex grammar. They also reported that they avoid using English in peer conversations because they associate it with authority and formality. 
students from "slums" resorted to Sheng because they were linguistically handicapped, hence they had difficulties expressing themselves in English.

On the contrary, students Mtile and Mchu in a focus group in Nakuru told me that Sheng speakers regarded the knowledge of Sheng highly and perceived "ghetto" as a site of "toughness" and "smartness" (Mtile and Mchu 10/2011, focus group-Nakuru). Mtile was from a low income neighborhood associated with Sheng while Mchu came from an affluent neighborhood associated with English. Even though Mchu distanced herself from Sheng later in the interview, she concurred with Mtile that speakers regarded the knowledge of Sheng as a social asset and an index of communicative efficiency. Specifically, Mchu claimed that speakers of Sheng could communicate and socialize with anybody because of the knowledge of Sheng. She contrasted speakers from "ghetto" with young people from her own neighborhood, claiming that the latter would "die" if taken to live in the "ghetto" because they were not used to hard life. Mtile on his part referred to "ghetto" as a "college of life" and elaborated that a person growing up in the "ghetto" would know a lot about life, hence he could survive in any other environment. Mtile summarized the various sentiments in his confession in the extract below (this section of the interview was in English):

Mtile: Ghetto is a college of life. [...]

MW: What does that mean?

Mtile: You know when you are in a ghetto, me personally I come from Bonde.

MW: Ehe, Bonde? Ehe,

Mtile: Bonde is a ghetto and you know being in Bonde which is ghetto, you interact with everyone. You learn to live with what you have. [...] You know they say that "He who has everything values nothing." But if you are coming from Bonde which is a ghetto, you will value what you have [...]. (Mtile and Mchu 10/2011, focus group-Nakuru). 
In Mombasa, student informants avoided the terms "slums" and "ghetto," possibly because of the stigma associated with these terms. Instead, they claimed that they lived in the "'hood," and were organized in small social groups each with a specific baze, i.e., a place where they met to socialize. Contrary to school authorities who associate these social groups with bad influence, speakers claim that they are sites of innovation and positive socialization. For example, in an individual interview with a student, Zainabu, she reported that young Kenyans in her neighborhood were organized in various social groups. She said that her social group spoke a distinct variety of Kiswahili cha Mtaani 'street Swahili', and that it was common for individual members to innovate new terminologies which were then embraced by other members if they found them "cool." In addition, she stated that members of the various social groups lived like "children" (meaning they loved and cared for each other) from the same neighborhood, which she categorically regarded as "'hood" rather than "ghetto." In this regard, she seems to make the claim that these social groups are for helping and taking care of one another. These claims are encapsulated in the extract below:

Zainabu: Mmh group yetu ni like, ok ni ya mayouths tu and hatutaki kusema tuna swag. Ni tu group tu ya kawaida that as in watu wa kusaidiana, to look out for each other. That's all. As in tuseme tu kama si unajua kama watoto wa neighborhood yaani. Let's say ni kama tu group ya hood sababu siwezi sema kwetu ni ghetto. Kwetu ni hood. (Zainabu 04/2012, interview)

Zainabu: Mmh, our group consists of youths and we do not like saying we have swag. It's just a normal group who are out to help and take care of each other. That's all. Let's say we interact as people from the same neighborhood because I cannot call it "ghetto." Our place is a "'hood." (Zainabu 04/2012, interview)

Similar references to "streets" and "ghetto" as social sites for "education," ingeniousness and cultural sophistication have been reported in the United States. For 
example, Brown (1972) who participated actively in the black civil rights activism in the United States describes his experience and that of his friends growing up black in the streets of Baton Rouge, Louisiana. He refers to the streets as a place where "young bloods" among the blacks get their education. He emphatically wonders why he went to school, since he acquired much of the knowledge that he employs in practical life from the streets. Specifically, he says that he "learned how to talk in the street, not from reading about Dick and Jane going to the zoo and all that simple shit. The teacher would test our vocabulary each week, but we knew the vocabulary we needed. They'd give us arithmetic to exercise our minds. Hell, we exercised our minds by playing dozens" (Browns 1972: 205). ${ }^{72}$

Alim (2004) also challenges the negative attitudes expressed toward "streets" by adherents of the dominant "standard language ideology" in the U.S. Specifically, he discusses how speakers of Hip Hop Nation Language (HHNL) regard "streets" as a locus of their desired identity and linguistic ingenuousness. According to Alim (2004: 391), although "the streets" can often have a negative connotation, "a close examination of the Hip Hop saturated streets of America reveals that the street is not just a physical space - it is a site of creativity, culture, cognition, and consciousness." Hip Hop musicians and other prominent figures in Hip Hop culture in the U.S choose to speak HHNL "to demonstrate their high degree of linguistic consciousness and in order to construct a street-conscious identity" (Alim 2004: 400).

By "owning up" the streets, Alim (2004) argues that these speakers are opposed to the white mainstream society's reference to "streets" as sites of crime and

\footnotetext{
72 This is a competitive verbal play/game characterized by exchange of ritual insults among peers to generate humor among other social functions. It is also called sounding. It marks the winner as "smart" and "creative." I will discuss Dozens in more detail later in the chapter.
} 
"black" as dangerous among other negative attributes. In the process, members of the HHN (who are predominantly African American), redefine what it means to be "Black" using the same terms used by whites to demean them though in positive ways. The HHN consists, by its own classification, of members of the "street," who are socioeconomically disadvantaged and opposed to the latent bias that the mainstream American society has against them. For example, Alim (2004: 393) reports how Bahamadia, a rapper of Philadelphia, praised fellow rappers for their efforts to "“explore the English language and [...] try to push the boundaries and go against the grains of it." In this context, Alim argues that by creating their own languages, speakers of HHNL can no longer be defined by a linguistic standard to which they no longer submit.

While adherents of the "standard language ideology" often misconstrue this ingenious manipulation of the "standard language" as ignorance and linguistic “errors," Alim (2004) and Holt (1972) interpret it as speakers' intentional disregard for linguistic icons and grammar in the dominant language, hence a form of social resistance. The various claims about how HHNL manipulates the standard indicate that speakers of HHNL are aware of their antagonistic relationship with those of the standard, hence their language choices are ideologically mediated. In the following section, I examine the rebellious nature of Sheng against the grammar of the standard.

\subsubsection{Nonstandard vs. standard grammar: stretching the limits}

Speakers of Sheng also contravene the "standard language ideology" by stretching the grammar of Standard Swahili and English. For example, Standard Swahili marks past tense using the morpheme - $l i$ - before the verb root. Thus, the past 
tense of shika (hold) is -lishika (the hyphen at the beginning marks the place for the pronominal subject). In Sheng, however, speakers appropriate the English tense marker '- $d$ ', hence the past tense of shika will be shikad. In one of the focus groups in Nakuru, I asked members to explain the meaning of "shrubbing," and why speakers pay attention to it. One student, Katuzo, responded that "'Shrubbing' ni kutamka a word the way it's not supposed to be tamkwad", 'Shrubbing is pronouncing a word the way it's not supposed to be pronounced' (Praise and Worship focus group, 11/2011--Nakuru). The table below shows the morphological structure of the word tamkwad, which is derived from a Swahili verb in passive form but its tense is marked by suffixing the English past tense marker '- $d$ ':

\begin{tabular}{|c|c|c|c|}
\hline Tamk- & $-\mathrm{w}-$ & $-\mathrm{a}-$ & $-\mathrm{d}$ \\
\hline $\begin{array}{c}\text { Verb stem } \\
\text { pronounce' }\end{array}$ & $\begin{array}{c}\text { passive } \\
\text { suffix }\end{array}$ & verb ending & $\begin{array}{c}\text { English Past } \\
\text { tense marker. }\end{array}$ \\
\hline
\end{tabular}

Speakers of Sheng and lugha ya mtaa also seek to challenge the dominant "standard language ideology" by violating the norms for formal speech in Kenya. So, what are these norms and how do speakers engage them? I focus on this question in the following subsection.

\subsubsection{Formality vs. informality among speakers of Sheng and lugha ya mtaa}

School as a formal setting prescribes formal discourse with regard to language use and associated behaviors such as manner of dressing. According to Irvine (1979: 774), formality is a broad analytical tool that has been used by scholars to mean different things. However, she identifies two senses of formality which I find relevant to the current discussion. Though I discuss them each at a time, they are closely intertwined. The first concerns properties of a communicative code which she 
describes as follows. Formality as an aspect of code, i.e. "formal style," emphasizes predictability of structured discourse. That is, the discourse is subjected to extra rules or some greater elaboration of rules which reduce the variability (deviation from the norm) and spontaneity of speech. Some of the structuring regulates phonology, syntax, use of particular sets of lexical items, turn taking and various aspects of intonation like speed of talk and loudness. Irvine's (1979: 774) second sense of formality concerns properties of a social situation which "may have something to do with a prevailing affective tone, so that a formal situation requires a display of seriousness, politeness, and respect." An example is the use of "respect vocabulary" and "formal etiquette" among the Trukese and Ponapeans in their ways of speaking as a display of politeness and marking the formality of the situation (Fischer 1972, cited in Irvine 1979: 774).

As mentioned in Chapter Four, the two schools in my study provided students with a printed set of rules and regulations at the time of admission. Teachers also communicated some of these rules verbally during school assemblies and other forums. One category of rules outlined the prescribed formality in students' conduct such as language use, manner of dressing and social relations with other members of the school, including visitors. Vikapuni School in Mombasa had the following rules relevant to this discussion, in addition to those cited in Chapter Four:

\section{CODE OF CONDUCT.}

1. Except for Swahili classes, English is the ONLY language to be used on campus by students, as well as teachers. ${ }^{73}$

2. All students are expected to show courtesy and be of mannerly behavior. To be respectful, smart and clean both in thought, word and deed at all times.

3. As a general rule, all students should stand when any member of staff or any visitor enters class. This should be done promptly and without noise.

\footnotetext{
${ }^{73}$ As mentioned in the earlier chapters, this rule was revised to read "SPEAK ENGLISH AT ALL TIMES," a few days into my research.
} 
4. The words, "Sir and Madam," should be used by all pupils on all occasions when they speak to any member of staff.

5. It is proper for ALL PEOPLE to use the terms, "Please and Thank You" when making a request or when granted a request. This includes teachers as well as students.

6. It is extremely rude not to answer when spoken to by a teacher.

7. Channels of communication: Class Prefect - Class Teacher - Teacher on Duty -Deputy Principal -- Principal.

8. The name the student gives at this school and uses must be the same as appears on his KCPE certificate.

In Someni School, the printed school rules and regulations did not mention a language policy. As a result, different teachers interpreted it differently. Some said that the policy required students to speak English and Standard Swahili, others said that students should speak English four days a week and Swahili on Friday. The rest claimed that the school did not have a language policy, and that was why students spoke Swahili and Sheng most of the time. However, in addition to the rules mentioned in Chapter Four, the school prescribed that:

1. Students will behave in a proper manner at all times. They will not do anything which breaks the laws of Kenya.

2. Students must not circulate or put up notices unless permitted to do so.

My study found that speakers of Sheng and lugha ya mtaa contravene these norms for formal speech in various ways. I will first address formality with regard to the attributes of the communicative code in the following subsection.

\subsubsection{Formality as an aspect of code}

One of the underlying motivations of standardizing a language is to enhance greater intelligibility because once a language is codified, it is more likely to be shared by a larger group of speakers than in the case of highly dynamic nonstandard language varieties such as Sheng. Speakers of Sheng and lugha ya mtaa value unintelligibility rather than intelligibility in their communication and this preference is driven by speakers desire to exclude unintended audiences while promoting in-group 
solidarity. For example, in an attempt to differentiate between Sheng and language mixing, students Mtile and Mchu said that Sheng enabled speakers to hide meaning whereas language mixing did not (Mtile and Mchu 10/2011, focus group--Nakuru). Similarly, recall that student Myumba reported that speakers employed Sheng mainly to communicate in-group secrets. In this regard, Myumba claimed that he could communicate with friends in Sheng in my presence, but I would not decipher what they were saying. He claimed that he and his brother could speak Sheng in the presence of their mother, but she would not understand what they were saying (Myumba 03/2012, interview).

These claims raise the following two questions: are the people being excluded part of the conversation or they are just present while the conversation is going on? Do these people get excluded in actual practice, or is it the language ideology of Sheng speakers that leads to the perception that non-speakers would not understand the Sheng? According to Goffman (1981), different forms of talk such as conversation, lecture or soliloquy, have different categories of participants. However, he argues that the traditional paradigm of analyzing talk is inadequate because it assumes that only two parties (speaker and listener), are involved at any given moment. That is, "these two individuals are the only ones who know who is saying, who is listening, what is being said, or, indeed, that speaking is going on--all aspects of their doings being imperceivable by others, that is 'inaccessible"” (p.129). Instead, Goffman proposes a more elaborate paradigm for analyzing talk, which opens up an array of structurally differentiated possibilities, establishing the participation framework in which the speaker will be guiding his delivery. He says that: 
The process of auditing what a speaker says and following the gist of his remarks-hearing in the communication-system sense-is from the start to be distinguished from the social slot in which this activity usually occurs, namely, official status as a ratified participant in the encounter. For plainly, we might not be listening when indeed we have a ratified social place in the talk, and this in spite of normative expectations on the part of the speaker. Correspondingly, it is evident that when we are not an official participant in the encounter, we might still be following the talk closely, in one of the two socially different ways: either we have purposely engineered this, resulting in 'eavesdropping,' or the opportunity has unintentionally and inadvertently come about, as in 'overhearing'. In brief, a ratified participant may not be listening, and someone listening may not be a ratified participant. (Goffman 1981: 131-32)

According to Goffman, talk is not limited to utterances but involves other forms of communication such as eye contact, touch and gestures. He says that bystanders sometimes rely on these cues to follow or decipher all or part of what is being said; hence their presence in any form of talk should be taken as the rule, not the exception. In the case of Sheng, speakers are usually aware of these adventitious participants, and therefore employ different strategies to enhance unintelligibility. For example, Mrs. Tajiriba from Nakuru reports how she boarded a matatu one morning during rush hour and sat next to the manamba. A few minutes into the journey, she heard the manamba say “changamka! changamka!”, 'cheer up! cheer up!’ Mrs. Tajiriba says that she was not sure who the manamba was addressing, and even if it were the passengers, she wondered why they needed to cheer up. Shortly thereafter, she realized that the matatu was driving really fast, suggesting that the manamba's use of "changamka changamka" was a coded message, asking the driver to drive faster, possibly to enable them to make more trips (Mrs. Tajiriba 11/2011, casual conversation).

Students reported that they value the ability to communicate fast, hence peers perceive speakers who use full words and long sentences as boring and "uncool." This is consistent with the existing literature that interprets youths' general desire for 
"shortcuts" as a marker of trendiness and sometimes "street smartness," especially when dodging police and other authorities (Ogechi 2005; Samper 2002). According to Samper (2002), speakers of Sheng feel obliged to be speedy in their articulation to ensure that only the targeted audience gets the message. So, what strategies do speakers employ to enhance desired unintelligibility?

Speakers employ different innovative strategies such as coining new lexical items, or manipulating the form and meaning of borrowed and existing material (Githinji 2006; Ogechi 2005). For example, at the level of lexicon, speakers of Sheng in Nakuru told me that they coin new innovations such as tintole 'darkness' and kadete 'act of talking too much', mentioned earlier. These speakers also employ syllabic reversal to generate new lexical items as in bwaku mentioned as an example of Sheng by student Mpenda from Nakuru (Mpenda 9/2011, casual conversation). The same is true of mjamo given by Mr. Nyachae from Nakuru to demonstrate the unintelligible nature of Sheng. Bwaku and mjamo are generated by reversing the syllables of the Swahili words kubwa 'big', and mmoja 'one' respectively. Even though their meanings are not altered, it would be hard for the uninitiated to decipher them when used in Sheng, because the new form would make them appear and sound different.

In addition, speakers employ similar innovations at the semantic level, expanding the meaning of an expression to include nuances not captured in the source language or altering its meaning altogether, as in the case of the expression “changamka changamka" mentioned above. In Mombasa, speakers of lugha ya mtaa reported that they employ mafumbo 'metaphors', which make their conversations indecipherable to non-speakers, and also differentiates its speakers from those of 
Sheng, which they associate with Nairobi. For example, the term kuzuka given as an example of lugha ya mtaa by Mwanashehe in the following extract is also found in Standard Swahili, meaning "to break out" or "to emerge." However, Mwanashehe says that speakers of lugha ya mtaa use it to mean "to show up" as demonstrated below:

1. MW: Kuna tofauti kati ya lugha ya mtaani and Sheng?

2. Mwashehe: lugha ya mtaani na Sheng? Huwa yategemea. Kama hapa na Nairobi, sisi tunavyoongea mtaani itakuwa ni similar but si like hao wa Nairobi. Wao wanatalk more.

[...]Kule wanatumia Sheng sana si kama hapa. [...]

3. MW: What are some of the words unaweza toa kama mfano wa lugha ya mtaani ya Mombasa?

4. Mwashehe: Mambo vipi? Poa. Mbona jana haukuzuka? Yaani, haukutokea.

5. MW: Ooh kuzuka?

6. Mwashehe: Eeeh, ni kutokea. Yaani umepotea kweli. Kama hivo. [...] yaani inakuja kama mafumbo.
1. MW: Is there a difference between Sheng and 'street language'?

2. Mwashehe: 'Street language' and Sheng? It depends. Like here and Nairobi, the way we speak in the streets would be similar but not like those of Nairobi. They talk more [...]. There, they use Sheng a lot, it is not like here. [...]

3. MW: What are some of the words you would give as examples of the 'street language' of Mombasa?

4. Mwashehe: How are things? Cool! Why did you not emerge? That is, why did you not show up?

5. MW: Ooh kuzuka?

6. Mwashehe: Eeeh, it is showing up. That is, you have really been lost [it's rare to see you nowadays]. Like that [...]. That is, it [street language] comes out like metaphors.

(Mwanashehe 01/2012, interview)

My field observations indicate that the most common manipulations are done when referring to figures of authority such as teachers, parents and police or when discussing prohibited endeavors such as sex or drugs. For example, recall Nakuru students' use of Bokono to refer to their Principal. Similarly, students in Mombasa reported that they referred to the police as mwera, a term they adopted from manamba. Indeed, the common association of Sheng with manamba contributes to its attraction to many young people as a language of resistance to authority associated with the "streets." Even though such manipulations are mainly aimed at excluding non-speakers, Sheng speakers also use them to assert authority in language or areas that authorities do not comprehend, thus symbolically challenging the existing social 
order. For example, two teachers (one in Nakuru and the other in Mombasa), reported how they usually got frustrated when their children spoke Sheng in their presence. The children first laugh at the parents for not comprehending, but later teach them the meanings of the words used. In this context, I argue that young people assume the authority and power to educate their parents, but since Sheng is highly dynamic, parents remain permanent students of Sheng. This situation accords young people some symbolic power which seeks to challenge the established social order (see Bourdieu 1977).

Figures of authority such as teachers or parents disparage these nonstandard language varieties because of their secretive nature, arguing that only "dodgy" fellows and gangsters speak like that (Githiora 2002). As already noted, these authorities also characterize speakers of Sheng as culturally eroded while referring to Sheng itself as “errors” or “corrupted” Swahili and English (Momanyi 2009; Mutiga 2013).

According to Mutiga (2013: 12), "Sheng has therefore been blamed for raising a Kenyan generation devoid of culture and cultural values. It has also been blamed by school teachers and parents for interfering with the learning and mastery of both English and Kiswahili, the official languages in Kenya." Conversely, I argue that what school authorities regard as "errors" is socially meaningful to speakers. In fact, some scholars like Kangethe-Iraki (2004) and Ogechi (2005) disagree with teachers' assessment, and regard linguistic innovations like truncated or shortened word forms in Sheng as markers of linguistic creativity and cognitive efficiency. Specifically, Kangethe-Iraki (2004: 65) argues that:

Owing to the complex linguistic heterogeneity characteristic of Kenyan cities, a young mind that is juggling with so many languages in contact, and mastering none, is likely to weave a composite system from the resources at its disposal to communicate. The traits of Sheng outlined in [the author's] discussion signal such 
linguistic creativity. Extensive borrowing, lexical and syllabic modifications, and idiosyncratic sound systems point to cognitive dynamism.

While "standard language ideology" does not encourage individual speakers to coin new words, I observed that Sheng speaking networks regard innovators highly. Innovators therefore take a lot of pride when their innovations are embraced by other youths. For example, in one of the focus groups that I conducted with male students in Nakuru, one student claimed that the word kadete (act of talking too much) was coined by a close friend and has become common in peer conversations (Form Three focus group, 10/2011--Nakuru). According to Bakari, peers recognize his friend as one of the most knowledgeable speakers and innovators of Sheng. They call him Kadete not only to identify him with this particular innovation, but to acknowledge his status in the Sheng speaking network. When I asked whether it was all right to invite Kadete to the focus group, all the group members applauded the idea and excitedly expressed that the presence of Kadete would make forthcoming group meetings more productive and "livelier." When Kadete subsequently joined us, other members not only appeared happier, but always sought his approval when talking about Sheng.

The reactions by other students to Kadete's presence confirmed the latter's authority in the Sheng speaking network. In this context, I argue that taking pride from a language variety that is disparaged by the authorities challenges the official ideology that values only the standard language. From a theoretical point of view, while use of a standard language is said to generate overt prestige, use of nonstandard language varieties such as Sheng generates covert prestige (Labov 1966, 1972). The difference is that while overt prestige is associated with a conformant identity in favor of the standard, covert is associated with an in-group that does not enjoy prestige in 
the wider society, and its use conveys symbolic meanings such as rebelliousness, which is valued by that in-group. The two forms of prestige are earned in different "linguistic markets" (Bourdieu 1977) that have opposing language ideology.

Similar conflicts in ideology between adherents of the standard and speakers of competing variants are witnessed both within and outside Africa. For example, Kiessling and Mous (2004) argue that the lexical and other linguistic manipulations characteristic of the African urban youth language varieties are not linguistic "errors" but innovations highly valued among speakers. In the United States, Lippi-Green (2012) reports how dominant institutions such as schools and the mainstream media simultaneously promote the standard while they disparage other language varieties such as African American Vernacular English (AAVE). Teachers describe nonstandard varieties in pejorative terms, such as "sloppy", "ignorant," and language full of "errors" (Pullum 1999). However, Alim (2004) reports that speakers of these nonstandard varieties such as Hip Hop Nation Language (HHNL), which is based on African American Vernacular English, do not subscribe to all the ideals of the standard.

At the semantic level, HHNL speakers claim that they do not rely on the meanings given in the Standard English dictionary, but instead take pride in their innovations especially if embraced by large numbers of speakers. In this regard, HHNL speakers not only create entirely new words, but also redefine or alter existing ones. For example, Alim (2004: 397) says that the HHNL word politickin to the uninitiated appears as misspelled "politicking," which the Merriam Webster dictionary defines as engaging "in often partisan political discussion or activity." In HHNL, however, politickin differs in form and meaning from "politicking." Speakers 
use it to mean speaking about political issues specific to the black community or the act of building a relationship with a female. According to Holt (1972: 154), inversion of form and meaning in communication among black people in the U.S. is informed by speakers' desire to make "any word of denigration used by the power group take on the shade of meaning known only to the inverter... Inversion therefore becomes a defensive mechanism which enables blacks to fight linguistic, and thereby psychological, entrapment."

The various examples discussed above demonstrate how speakers of Sheng and lugha ya mtaa contravene formality as an aspect of code. In the following subsection, I discuss how speakers contravene Irvine's (1979) second sense of formality.

\subsubsection{Formality as properties of a social situation}

A second sense of formality discussed by Irvine (1979) requires speakers to display seriousness, politeness, and respect. However, that is not the case with some speakers of Sheng and lugha ya mtaa. They seek to challenge the dominant official language ideology by employing vulgarities and other socially inappropriate words or expressions in peer conversations. But in what sense do these vulgarities challenge the official language ideology? According to Bakhtin (1968: 188):

Abuses, curses, profanities, and improprieties are the unofficial elements of speech. They were and still are conceived as a breach of the established norms of verbal address; they refuse to conform to conventions, to etiquette, civility, respectability. These elements of freedom, if present in sufficient numbers and with a precise intention, exercise a strong influence on the entire content of speech, transferring it to another sphere beyond the limits of conventional language. Such speech forms, liberated from norms, hierarchies, and prohibitions of established idiom, become themselves a peculiar argot and create a special collectivity, a group of people initiated in a familiar intercourse, who are frank and free in expressing themselves verbally. 
In Nakuru, I observed that a student would find peers in a small group either in the playing field or along the classroom corridors and greet them using expressions such as "semeni wajinga” ,'say something, you fools' or "vipi wachizi?", 'what's up you crazy guys?' or “semeni Mafala”, 'say something, you idiots'. School authorities consider such language socially inappropriate, hence punishable. Conversely, in a recent follow-up interview over Facebook with a female student from Nakuru, Mandumo, she explained that such greetings are used only among speakers who know each other very well; hence peers do not expect interlocutors to take offense. In this regard, the shared context of use among speakers normalizes these vulgarities which are otherwise considered socially offensive in the wider society. In addition, Mandumo said that there are certain places or contexts where speakers accord new and positive meanings to the words which are offensive in their source languages. These differences are captured in the following extract:

MW: Hebu nieleze kwa nini youth husalimiana hivi: semeni mafala, semeni wajinga na vipi wachizi?

Mandumo: Okey, hizo ni salamu za wale mayouth wamezoeana, as for ladies unaeza skia wengine wakisalimiana, hey my bitch. But mchizi wangu, place kama coast ama TZ it means my friend. (Mandumo 03/2013, Facebook interview)

\author{
MW: Please tell me why youth in \\ Kenya greet each other using \\ expressions such as: 'say something, \\ you fools', 'say something, you idiots', \\ and 'what's up you crazy dudes?' \\ Mandumo: Ok, these are greetings used by youths \\ who know each other very well. For female peers, \\ you might hear some of them greet each other, \\ 'hey my bitch'. But in Coast or Tanzania, the \\ expression "mchizi wangu," means 'my friend'
} (Mandumo 03/2013, Facebook interview)

I recorded similar sentiments from young Kenyans in Mombasa, but I observed that they curse and employ vulgarities in their conversations more than their counterparts in Nakuru. This challenges the local cultural frameworks because Mombasa is associated with Swahili civilization, which has been strongly influenced by Islam with its strict moral codes. For example, recall from Chapter Four that Mr. Naibu, the 
teacher from Mombasa, claimed that his school's English-only policy was informed by the desire to raise a cultured society. He reported that the policy ensured that students used "stable" words, which allowed them to stick to the norms of civilization. He also said that the policy discouraged students from using Swahili or mother tongue, because school authorities associated these languages with noise, violence and vulgarities.

However, I observed that students regard use of vulgarities in peer conversations differently, and sometimes attach positive meanings to it. For example, I asked participants in one of the mixed-gender focus groups in Mombasa to comment on my earlier observation that people in Mombasa often used abusive language. They responded that cursing and use of vulgar language were "normal" in Mombasa. One of the group members called Mkotho reported that he knew two boys in the school who were twins and were always cursing each other. Student Zainabu who was also in this focus group said that she and her sister cursed just like the two boys, and gave examples of the kind of vulgarities they used. She would call her sister nzi shoga 'gay house fly' and her sister would call her "mjusi kafiri kiwete”, 'a pagan crippled lizard'. Zainabu reported that neither she nor her sister took offence. Instead, the more they cursed, the more humorous their conversations became. She considered cursing to be normal and she regarded it like another form of Sheng involving insults only. Below is an extract showing some of these views:

Zainabu: Aah [...] kutukanana as in kwangu hiyo ni kitu ya kawaida. Ni as in, ni kama. Saa ni kama pia tuseme ni Sheng ingine ambayo ni ya matusi peke yake tuseme. (Form Four focus group 2, 11/2011--Mombasa)

Zainabu: Aah [...] insulting each other to me is something normal. It is like another Sheng comprised of insults only. (Form Four focus group 2, 11/2011--Mombasa) 
Even though these speakers claim that cursing and use of vulgar language is normal to them, they report that newcomers find it strange and offensive at first, but gradually get used to it and embrace it. This is because use of vulgarities as a style of speaking expresses in-group solidarity, and marks the speaker as "tough" and "cool" among peers. Therefore, speakers who do not curse or those who take offence when such language is used against them are said to be "down," meaning "uncool." They are also ridiculed and told to stop pretending that they are "holy." Student Mkotho, for example, narrated how he went home for half-term (mid-semester break) and interacted with a friend from a different high school who was cursing a lot. Since Mkotho was not used to such language, he took offense when his friend cursed him. However, his friend scoffed and told him to get used to such language since he would soon be using it as well. These views are highlighted below:

\begin{abstract}
Mkotho: [...] Eeh those who are not using that abusive language, they are also being seen as people who are down. You see? Like also me, I like speaking from experience, as in during the half term, we went home then I met a friend of mine also from another school. You see? So as in as I was with him as in when we were interacting then I don't know he told me what, then he told me "kuma [ma]mako" ['your mother's (expletive)']. As in I was so offended and I told him "eeh, whats up bana?" Unaona? ['You see?']Then he told me ati "No don't be mad at me. This is what we do." So as in I asked him "What?" Then he was telling me as in when he saw that am not into that then he started telling me that "you stop pretending! Nowadays you are taking yourself to be so holy. These are the things that we do here. Even when you, when you close you will also adapt to these changes." So, if you don't as in if you don't try into that, they are seeing you as if you are somebody who is down or as if you are pretending. (Form Four focus group 2, 11/2011--Mombasa)
\end{abstract}

I also observed that young Kenyans engage in a competitive and trangressive verbal duel that they call mchongoano. According to Githinji (2006), mchongoano discourse is mediated by Sheng. Just like the game of dozens in the U.S, mchongoano discourse is produced in the streets and employs vulgar language and taboo themes. It is therefore socially situated in 
opposition to adult middle-class norms (Githinji 2006). ${ }^{74}$ Both duels normalize the vulgarities used, and sometimes give them positive meanings.

But what makes use of vulgarities in young peoples' conversations normal, and how does this normalization articulate with social norms promoted in the wider society? According to Labov (1972: 289):

Many sounds ['ritual insults'] are obscene in the full sense of the word. The speaker uses as many "bad" words and images as possible--that is, words subject to taboo and moral reprimand in adult middle-class society. The originator will search for images that would be considered as disgusting as possible: "Your mother eat fried dick heads." With long familiarity, the vividness of this image disappears, and one might say that it is not disgusting or obscene to the sounders. But the meaning of the sound and the activity would be lost without reference to these midlle-class norms. Many sounds are 'good' because they are 'bad'-- because the speakers know that they would arouse disgust and revulsion among those committed to the 'good' standards of middle-class society.

The game of dozens (which was called "sounding" by the youths Labov recorded in New York City in the early1970s) is organized in small social groups of close friends. According to Labov (1972), participants' conduct is governed by social conventions, which require them to depersonalize the situation in order to successfully remove the dangers of a face-to-face confrontation. In this regard, he observes that sounds are directed at close peers but by convention should not denote attributes which are either factual or actually possessed by targeted persons. However, just like student Mkotho said about newcomers finding use of vulgarities strange and offensive in the Kenyan context, Labov (1972: 314) observes that "the ritual convention can break down with younger speakers or in strange situations - and the dangers of such collapse of ritual safeguards are very great." Therefore,

\footnotetext{
${ }^{74}$ For examples of Mchongoano, see: (a) http://freefunny-jokes.blogspot.com/p/best-mchongoano$\underline{\text { 2011.html (b) https://www.facebook.com/kenyanjokes/posts/349002365181843 }}$
} 
participants are expected to remain in control of their feelings lest they lose their face. Participants reward winners using overt comments and reactions such as prolonged laughter or repeating the sound to give it more weight. At the end, the winner is socially constructed as culturally sophisticated, "creative" and "smart."

Similar transgressive use of vulgarities against mainstream middleclass norms has been reported in Hong Kong among young working-class speakers of Cantonese. They employ chou-hau (vulgar speech) as verbal art in their conversations (Lin 2009). According to Lin, Cantonese is the mother tongue of the majority of Chinese in Hong Kong, but is not recognized in formal settings. However, it is an important language in pop culture. English, the language of the former colonial power, remains the most important language and a tool for social mobility. However, the majority of workingclass children in Hong Kong unlike their middle-class counterparts live in environments where use of English is limited, and thus the language largely remains out of their reach. Consequently, Lin (2009: 162) reports that "the English classroom often becomes a site for their local struggles and oppositional practices involving a great deal of creative work in the form of Cantonese verbal play. And this verbal play capitalizes on the use of Cantonese chou-hau expressions to create a transgressive, subversive effect." For example, she reports how a male student in an English lesson in Hong Kong responded to a teacher's question using a euphemistic expression in English whose meaning was informed by Cantonese chou-hau. Fellow students who deciphered the underlying cultural logic laughed loudly, because 
they recognized this student's clever and transgressive rendering of a taboo Cantonese chou-hau expression as an answer to the teacher's formal question.

Cantonese chou-hau also conjures explicit sexual images. As a result, Lin (2009: 163) reports that the wider society in Hong Kong regards use of chou-hau as highly taboo so much that "a mere uttering of Cantonese vulgar word or expression constitutes a highly marked, trangressive act, violating middle-class etiquette and sensibilities.” Interestingly, Lin observes that speakers use of chou-hau is both deliberate and "natural." It is deliberate because they are aware of the wider society's negative attitude toward chouhau, hence their use of chou-hau is often supposed to arouse unease and contempt from a middle-class audience. On the other hand, use of chou-hau is “natural” because it makes speakers' conversations lively and also defines working-class people in Hong Kong. These speakers claim that the language reflects the everyday reality of their lives such that telling them not to use it amounts to denying them their actual identity.

Even though there are significant social differences between Hong Kong and Kenya, I argue that Cantonese speakers' use of chou-hau to transgress middle-class norms and to negotiate group identity provides a useful lens for interpreting the social motivations behind the use of vulgar speech among speakers of Sheng and lugha ya mtaa. In addition to contravening the norms of formal speech in Kenya, I observed that speakers of Sheng and lugha ya mtaa also challenge prescribed manners of dressing and body hexis. I focus on this issue in the following subsection. 


\subsubsection{Non-linguistic dimensions of Sheng and lugha ya mtaa}

Speakers of Sheng and lugha ya mtaa also employ non-linguistic "meta-signs" such as mode of dressing and walking style. These include piping their pants, styling their hair or wearing shoes associated with media celebrities, in order to make them more appropriate for negotiating their desired distinct identities. The various "stylizations" challenge the prescribed body hexis with regard to manner of dressing both within and outside the school. Recall from Chapter Five that each of the two schools where I conducted my research prescribed particular school uniforms for both girls and boys. The teachers I talked to elaborated that school authorities associated the school uniform with decency, and used it to track students both within and outside the school. These authorities insisted that students should be in proper school uniform at all times. Also, they prohibited students from modifying their uniforms.

Although students acknowledged that they understood why they were required to wear a school uniform, they reported that they found it "unfashionable" and so they constantly sought to modify it to fit their tastes. These sentiments are captured well in the following extract from student Tinga's focus group:

MW: Let's come to the school uniform. Tinga, why do you think students are told to wear uniforms?

Tinga: Ooh, before you ask me why do I think first, why don't you ask me whether it is appropriate?

\section{MW: Go ahead.}

Tinga: Personally, I despise school uniforms. I really wish that people would wear the, actually I doubt whether I have been free, coming again back to freedom to wear whatever they choose, Yah? You know that one at least creates confidence in most of 
us and confidence is a very important factor in how one conducts himself and also is a factor that determines our character. Why I think that uniform is mostly recommended to students is because they want to create equity. [...] So, actually uniforms are equalizers; they have actually made us all equal. At the same time, not most youths go for them. [...] Decency in youth perspective has to do with eeh how one, you know, appears in terms of sexual arousement, yah? Mostly youth connect the way they wear their clothes with sex. And also they connect it with, eeh, you know, that attraction, yah? I don't know how to call it. Actually is some sort of attraction which of course is natural for every youth. Every youth has that kind of feeling, yah? So they believe that by wearing such, you know, seductive clothes if I may call them, they get aah, first they get at par with the current fashion. Second of all, they are noticed and third of all you know they feel that most of their freedom you know is expressed and they feel that their exuberance is just taking place. (Form Four focus group 1, 01/2012--Mombasa)

Recently, though outside my two research sites, girls from several

Kenyan high schools protested that they should be allowed to wear "shorter"

and "more appealing" skirts as part of the school uniform rather than long

skirts, which they regarded as outdated and unfashionable, a sentiment with

which the immediate former Minister of Education concurred. ${ }^{75}$ In a video

clip, the Minister challenged parents and teachers in one of the affected

schools that he visited, and called upon them to respond to students desires.

He said:

Mimi nakubaliana nao ['I agree with the students']. Why are you dressing a school girl like a nun? What do you have in mind? God has created her, allow our children [students clap]. Si mnaona? ['You see?']. Be modern like [Minister's name]. These girls don't want to become nuns; these girls want to become Kenyan citizens. If you are a schoolgirl and you don't like your uniform, don't break someone's legs, just talk to me, and I will change the attitudes of the teachers and the parents. ${ }^{76}$

The Minister's statement above speaks to issues of nationalism and modernity

as envisioned by the state. The incident is consistent with my argument that

the stylistic nature of Sheng and lugha ya mtaa, and their popularity among

young Kenyans is a non-violent protest through which they contest and

\footnotetext{
${ }^{75}$ Here is a video clip showing the Minister making these remarks: http://www.youtube.com/watch?v=IXi2sZIQnYandfeature=related

${ }^{76}$ Refer to this page for more details: http://www.rnw.nl/africa/article/kenyan-schoolgirls-skirts-sparkcontroversy
} 
challenge official ideology about the appropriateness of language and other aspects of behavior. In this context, speakers also assert their agency to define themselves using preferred badges of identity (Wairungu 2013).

Besides school uniform, schools also prescribe how students should hold their body. For example, recall from Chapter Five that the Mombasa school prohibited students from keeping their hands in their pockets, or standing arms akimbo when talking to teachers or school visitors. The school also had another rule stating that: "assemblies are important for communication and must be respected. Students should not talk, laugh, play or stand carelessly in the assembly; any misdemeanor in these places shall be regarded as gross insubordination." The two rules indicate that respect for authority is expressed or measured using a speaker's language and his body.

However, I observed that some speakers had adopted performative walking styles, which not only distinguished them from peers, but suggested resistance to prescribed body hexis. For example, I asked students in Tinga's focus group above whether there were individual students whose swag was well-known in the student community. They responded that they knew of a particular student in Form Three who walked in a manner other students found unique and entertaining. They said that he usually assumed that walking style whenever he was called by a teacher during school assembly. This drew attention from other students and made them laugh. I argue that they laughed because they associated his walking style with rebelliousness. These sentiments are captured in the extract below: 
Tinga: Yes there is another one when he is called, he walks like aah ...

Mkinango: Nani? ['Who?'] Kenso?

Tinga: Yah, yule wa form thirty? ['The one in form thirty?']. ${ }^{77}$ Especially when he walks, the whole school laughs and they clap for him. Yah, they know his characteristic unique behavior.

Mshie: About swag, yah, in our school mostly, [...], there are other students who can be identified with the way they walk. Like the boy that Tinga had said, that boy whenever he is mentioned, everybody will be attentive; they want to see the way he will walk, the way he will react. And there are some other girls, the way they just walk you will just know aah fulani wa fulani yuakuja ['so and so is coming']. Just the way they walk, the walking style. And then there is this kind of talking, most of the girls have got a swag of, in Kiswahili twasema kutendekeza sauti ['in Swahili, we say luring the voice']. As in, they don't talk in their real voices. Like now they talk na ile sauti eeh ['with that voice'].

(Form Four focus group 1, 01/2012--Mombasa)

The foregoing discussion has focused on the social practices that suggest speakers' resistance to the dominant official language ideology and local cultural frameworks. However, some of my research findings as mentioned earlier indicate that speakers of Sheng and lugha ya mtaa exhibit simultaneous resistance and conformity to the same. I discuss some of these aspects of conformity in the following section. I will also address a similar point in more detail in the next chapter.

\subsubsection{Speakers' compliance to the "Standard Language Ideology"}

Students Tinga and Zainabu who earlier projected themselves as fashion conscious and rebellious provide excellent examples of how speakers of Sheng and lugha ya mtaa conform to the dominant official language ideology and local cultural frameworks. I start with Tinga who wore a school shirt with tightened sleeves to emphasize his biceps and had visibly put in his pocket a pack of cut-outs of 200 Kenyan shilling notes secured with a rubber

\footnotetext{
${ }^{77}$ Forth Thirty is a coded reference to Form Three.
} 
band. A real banknote on top masked the fake bills but allowed him to project himself as a rich and "cash money" guy. However, his body hexis sometimes contradicted what he said verbally. For example, he claimed in the following extract that what youth called fashion was not fashion per se. Instead, it was a funny style which they thought was "cool." I attribute these contradictions partly to the fact that Tinga was the school captain (head boy), hence he sometimes became conscious of his identity as a senior prefect who ought to promote the officially sanctioned identity in his interactions with other students (and perhaps, with me).

\section{MW: Are they the ones we call celebs?}

Tinga: The celebs, exactly! The celebrities. So, when, lemme first talk about the boys; when the boys see people like Lil Wayne you know, wearing those skinny trousers but are meant for sagging. They are not meant to be put on the waist. They are meant to be put from below the waist. Yah, so they see him, you know, walking in some, you know, fashionable style which is not really fashionable style; it is funny style, you know, and they think it is really cool .

\section{MW: Is it fashionable among the youth?}

Tinga: Yah, they think it is fashionable just because the person is a celebrity and so they think it rocks. So, one would want to associate himself with this celebrity. So they will be going to the market and find the same type of clothing and walk in the same type of style, which is quite funny.

\section{MW: Do they want to be like him?}

Tinga: Yah they also want to be, feel like celebs.

(Form Four focus group 1, 01/2012--Mombasa)

I recorded similar contradictions from student Zainabu. In an

individual interview, she first projected herself as an enthusiast of Sheng and

Kiswahili cha Mtaani. She reported that she spoke a variety of Swahili which she described as a mixture of both the standard and the variety associated with the youth. She said for instance: “aamh, my Swahili is let's say mixed; both for class and, Ok, the one that youths talk nowadays. Tuseme Kiswahili ya 
mtaani”, 'let us say street Swahili' (Zainabu 04/2012, interview). She then added that the "street Swahili" that she spoke was similar to Sheng and was characterized by vocabulary that only speakers could comprehend. She narrated how she and her other friends prevailed upon her friend "who was a mtu wa English”, 'a person of English or who speaks English', to speak "street Swahili" in order to be like them. I interpret her embrace of Sheng and Kiswahili cha Mtaani as resistance because she is aware of the officially prescribed languages, but she chose to speak the unauthorized varieties associated with the youth and the "streets." This rebellious language ideology is also captured in her pejorative reference to her friend who spoke English as mtu wa English.

However, in the same interview, Zainabu reported that she usually advised peers to embrace Standard Swahili and English in order to excel academically. She criticized students who spoke nonstandard language varieties, saying that they affected their performance in Standard Swahili and English because they were full of taka taka za mitaani 'street trash'. She elaborated that speakers of these nonstandard language varieties found it hard to use the standard when doing exams. She concluded that speaking standard Kiswahili "is actually healthier and good for students and everyone actually" (Zainabu 04/2012, interview).

From another point of view, some speakers exhibit conformity to what Lippi-Green (2012) calls "the myth of non-accent," which is an effect of the dominant "standard language ideology." They claim that people go to school in order to replace their ethnic languages or associated accents with English. 
In Chapter Four, I noted that many Kenyans associate English--but not Standard Swahili--with formal education and intelligence. Some speakers of Sheng subscribe to this language ideology, hence perpetuating the hegemonic status of English in Kenya. Interestingly, even though speakers of Sheng and lugha ya mtaa express negative attitudes toward ethnic languages, indicating that they conform to the "standard language ideology," they at the same time innovate linguistic material from these languages for different social functions such as resistance and secrecy. For example, in Chapter Four, I cited teachers and students in Nakuru who said that speakers of Sheng changed the form and meaning of words borrowed from ethnic languages before they appropriated them in Sheng. Student Mtile, introduced earlier, gives practical examples from his experience in soccer in the following extract:

Mtile: Here in football utapata ukiambiwa mkate ['you will be told mkate'], and it doesn't mean mkate chakula ['loaf, as in food'], ni mcherenge ['it means you overtake him']. Ama unapata unaambiwa mtware yaani ['or you will be told mtware'] overtake, while in Kikuyu inaamanisha ['it means'] run shoulder to shoulder.

MW: [Laughs]

Mchu: [Laughs]

MW: Does it have the same meaning in Kikuyu and in Sheng?

Mtile: No.

(Mtile and Mchu 11/2011, focus group--Nakuru).

Despite the negative attitudes that speakers of Sheng and lugha ya mtaa have toward ethnic languages, they evaluated coastal Swahili accent positively, especially that of Kimvita. In Chapter Four, I noted claims by speakers of Sheng from both Nakuru and Mombasa that they found coastal Swahili "sweet" and "poetic"; student Zawadi even utilized it as a social asset when 
she visited her relatives in Nairobi. (This paradox is discussed in detail in the next chapter.)

Having established that speakers of Sheng and lugha ya mtaa employ them to challenge authority and to resist the "standard language ideology" promoted in schools, it is important to explore the role that gender plays in speakers' use of these styles. I focus on this issue in the following subsection.

\subsubsection{The role of gender in speakers' use of Sheng and lugha ya mtaa}

The existing literature on language and gender suggests that female speakers are more likely than male speakers to aim for standard language forms. For example, Trudgill (1974) conducted research in Norwich, England, where he asked his subjects to comment about their own speech, i.e. self-reporting. He gave his subjects two pronunciations of a linguistic variable and asked them to pick the variant that they normally used. Female subjects reported that they used the prestige pronunciation of certain speech sounds associated with the social class(es) above theirs. In the process, their speech tended towards hypercorrectness. Male subjects on the other hand reported that they used the pronunciation associated with lower social classes which generated covert prestige and marked them as "tough."

However, when Trudgill (1974) conducted direct observations on actual use of speech among the same subjects, he found that female speakers had "over-reported." That is, they did not use high prestige forms in actual speech as much as they had reported. Also, he found that the male subjects had "under-reported." That is, they did not use lower prestige forms as much as they had reported. The disconnect between 
reported and actual speech among male and female subjects supports the traditional view of women as being more likely to have social class aspirations than men.

The literature on language and gender in Kenya suggests that female speakers are more concerned with standardness and formality than male speakers. For example, Jagero and Odongo (2012) conducted research among lecturers and other members of staff in an ethnolinguistically diverse aviation college in Nairobi to establish how gender, age and rank influenced speakers' language choices. They noted that employees' rank (seniority) corresponded with their age. So the higher the rank, the older the employee and vice versa. These researchers found that the main languages used by the research subjects were English, Swahili, ethnic languages and Sheng. Speakers employed different codes depending on formality or informality of the context such as the topic of discussion. However, the researchers state that these languages had different levels of formality as follows: English was the most formal, followed by Kiswahili, Sheng and ethnic languages. They say that Sheng was regarded as more formal than ethnic languages because it is a mixture of English and Kiswahili. In this regard, they categorized English and Kiswahili as formal codes and Sheng and ethnic languages as informal.

Jagero and Odongo (2012) found that female speakers in all ranks used formal codes more than their male counterparts even when they engaged in code-switching. ${ }^{78}$ Also, the high ranked female speakers used formal codes more than the lower ranked ones. The high ranked female speakers preferred speaking Standard Swahili and English, and even when they code-switched, they employed standard forms of the two languages. Jagero and Odongo claim that their findings agree with Cheshire's (1998)

\footnotetext{
${ }^{78}$ Owing to the fact that Sheng is socially defined, I use the terms Sheng and code-switching here as used by Jagero and Odongo (2012).
} 
who found that women use standard codes more than men, and with Kanana (2003) who found that female speakers prefer English more than their male counterparts. With regard to informality, Jagero and Odongo (2012) found that the low-ranked speakers used Sheng more than high ranked speakers but the high ranked speakers used mother tongue more than the low ranked speakers. This particular finding contradicts their prediction about the informality of mother tongue. This may be due to the fact that Sheng is socially defined. Therefore, some subjects might have denied that they speak it because of the associated stigma. The contradiction may also be due to the researchers' failure to acknowledge that formality of speech in Kenya is contextual. As such, it is possible for speakers to employ official languages such as English in informal talk on the one hand, and employ unofficial languages in formal talk on the other. Despite this contradiction, their findings are still relevant to the present discussion.

According to Jagero and Odongo (2012: 369), "while the high rank speakers/senior age are more formal and standard, the low rank speakers/junior age are less formal and nonstandard." The researchers elaborated that the high ranked/senior age speakers prefer formal codes even when code switching as a way of expressing their high status solidarity. Also, their preference for mother tongue over Sheng on the one hand indicates their high level of confidence which comes with age and high rank. On the other hand, it indicates that they are conservative and proud of their ethnic languages, and that is why they use them in public settings even in the presence of non-speakers. Such ideological association of Sheng with young people erases (Irvine 2001) its use among adults. Previous research on Sheng reports that it is 
a male-dominated language for different reasons. For example, Samper (2002) reports that:

Women are not as devoted to Sheng as men.... [Y]oung women, in the interviews and in the questionnaires from the women's high school, said that they did not invent Sheng words. With only a few exceptions, women do not have the same emotional attachment to Sheng and do not benefit from its use as young men do... Men derive cultural capital from the use of Sheng, but women lose those benefits. Sheng for men is a source of interpersonal power, and women's avoidance of it reflects women's lack of power in Kenyan society.

Samper (2002) reports that female informants avoided identifying with Sheng because of the stigma associated with it. Some of them said that Sheng is associated with prostitutes, drug dealers, and poor people. Others said that Sheng is a language of the "streets." Besides the stigma associated with Sheng, Samper (2002) argues that local cultural frameworks also prohibit women from speaking Sheng. While echoing Friedman and Todd (1994), Samper (2002: 154) argues that "women in Kenya lack personal power because girls are socialized to be silent, subservient to men, and not to voice their needs." In this context, he interprets use of Sheng among men as a marker of "toughness," while it renders female speakers people of questionable moral values/standards.

In the schools where I did this study, authorities generally associated Sheng with youth, especially boys with discipline problems. These associations render use of Sheng among females and adults, including teachers, invisible. For example, recall from Chapter Five that Mr. Nyachae and Mrs. Kasisi from Nakuru associated Sheng with players of rugby, a game played by boys or men in Kenya. However, the data presented in this chapter contradicts such associations. Specifically, the discussion on speakers' use of Sheng in academic contexts indicated that there were teachers who employed Sheng when interacting with students. Also, most of the student informants 
that I talked to, both male and female, reported that they spoke Sheng rather than the officially sanctioned languages. In addition, contrary to previous research that associates Sheng innovations with male speakers, some of my research findings indicate that female speakers also innovate and their innovations are embraced even by male peers. In Mombasa, for example, student Zainabu reported that she coined fantabulous from the words fantastic and fabulous, and the new innovation was embraced by both male and female members of her social group (Zainabu 04/2012, interview).

Similarly, female speakers of these nonstandard language varieties sometimes engage in social practices that transgress or challenge local conceptualizations of femininity. For example, Cameron (1995) argues that gender is socially constructed rather than "natural." She observes that different societies prescribe certain norms of language use for men and women. In the case of women, she points out that their verbal conduct is important in many cultures, thus they are instructed in the proper ways of talking just as they have been instructed in appropriate dressing and other "feminine" kinds of behavior. Other scholars such as Holmes (1998) report that women in some cultures are expected to be silent, like children, hence they are there to be seen but not heard. In this regard, Holmes observes that any amount of talk from women seems too much, improper and annoying. She gives examples of proverbs from various cultures demonstrating how women are socialized to be silent beings.

However, recent work on language and gender, such as Eckert and McConnell-Ginet (2013) argues that even though gender is ascribed to us by 
our cultures from early childhood, it is not a static identity. Instead, it is a fluid, life-time process. As such, humans do not just reiterate what has been given to them. Instead, they enact gender through social practice. In other words, gender is performed: it is possible to find one individual, male or female, in a social group performing multiple masculinities or femininities respectively. In this context, Eckert and McConnell-Ginet (2013) caution that if men speak or dress in a manner culturally associated with women it does not necessarily mean that they are gay. Instead, they may be using language or dressing style to negotiate different kinds of masculinity. The same applies to women who dress or behave in ways culturally associated with men; it does not necessarily mean that they are lesbians. Instead, they may be using such cultural forms to negotiate different kinds of femininity.

In the course of doing my research, I observed that there were female students, in both Nakuru and Mombasa, whose behaviors and desired identities transgressed local conceptualizations of femininity. A good example was the student Zainabu. She was Muslim, commonly assumed to be a culture that requires women to be submissive to men, and also one that does not encourage young women to interact freely with their male counterparts. However, Zainabu's socialization contravenes culturally ascribed femininity. Outside school, she reported belonging to a social group that had more boys than girls. She described female members in ways that socially constructed them as "tough" and "independent," while characterizing other girls in her neighborhood as "proud" and "antisocial." Specifically, she claimed that the girls in her social group operated like "tomboys," meaning that they socialized 
with boys freely. She then described herself as a person who did not talk much but instead preferred quietly observing and listening to other group members, and only talked at the end.

However, Zainabu cautioned that her quiet demeanor should not be misconstrued to mean that she was shy. Instead, she told me that it was a deliberate strategy that allowed her to study what other members were saying, and then be able to give the final word. This claim suggests that she considered herself as having authority over other group members, including boys. This is possibly why she later said in the interview that other members referred to her as "Jenerari," which she glossed as 'Military General', a title associated with men in Kenya. The various characteristics of the female members of Zainabu's group challenge local understandings of femininity because they ascribe masculine attributes to women. These characterizations are encapsulated in the following extract:

1. MW: Na is this group comprised of both boys and girls ama iko aje?

2. Zainabu: Actually the group is comprised of more boys than girls.

3. MW: More boys than girls?

4. Zainabu: Yah. So far we are only two girls in the group coz pia inategemea unajua wasichana huwa si the same.

5. MW: Ni kweli.

6. Zainabu: Nayo group yetu tuseme ni ya wasichana ambao ni Matom boys kiasi kama mimi. As in saa mtu ambaye yuko social anaweza kuongea na kijana kitu chochote, wajua?

7. MW: Ni kweli. You are free!

8. Zainabu: Yah, sio wale wasichana wengine wakujidaijidai. So madame wengi wanakuwa recruited. Aah so far tuko tu wawili na mmoja ametravel. Saa hii niko peke yangu.

9. MW: Uko peke yako kwa ile?
1. MW: And is this group comprised of both boys and girls or how is it?

2. Zainabu: Actually the group is comprised of more boys than girls.

3. MW: More boys than girls?

4. Zainabu: Yah. So far we are only two girls in the group because it also depends. You know girls are not the same.

5. MW: That's true.

6. Zainabu: Let's say the group is comprised of girls who are tomboys like me. As in a person who is social and who can talk about anything with boys. You know??

\section{MW: That's true. You are free!}

8. Zainabu: Yah not the kind of girls who are full of themselves. So we recruit many girls but so far we are only two and the other one has gone out of the country. So, I am the only one left.

9. MW: So you are the only one in that? 
10. Zainabu: Iyo group yote. [...] As I mentioned earlier, nikiwa kwa group, I am a silent person. [...] kama kwa group, mimi huitwa Jenerari

11. MW: Mhh, in the Group

12. Zainabu: Yah, General. Like sema like cheo yaani. Saa badala ya kusema General wanasema Jenerali. Hata si Jenerali ni Jenerari.

13. MW: And now that that title is associated with men, mbona wakakuita Jenerari? Ama?

14. Zainabu: Mimi ni Jenerari kwa group coz mi hukaa huangalia na mwishowe mimi ndiye nitaongea. Mi huwa siongei. So mine is just to sit back and relax and watch what everyone is doing.
10. Zainabu: In the whole group. [...] As I mentioned earlier, when am in the group, I am a silent person. [...] I am called Jenerari 'General'

11. MW: Mhh, in the Group

12. Zainabu: Yah, General. It's a title. So instead of saying General, they say Jenerali. Even it's not Jenerali but Jenerari.

13. MW: And now that that title is associated with men, why did they call you General? Or how was it?

14. Zainabu: I am the General in that group because I usually stay silently but I am the one to talk at the end. I usually don't talk. So mine is just to sit back and relax and watch what everyone is doing.

(Zainabu 04/2012, interview)

Zainabu's socialization in school also transgresses local conceptualizations of femininity. For example, she reported that she preferred being alone or in the company of boys rather than girls. When I asked her to comment on her choice of friends, she said that she avoided hanging out with girls because they were "show offs" and since she was a "tomboy" and a person who did not like "show offs," it was very easy for her to fight such girls. In addition, she claimed that she was a "tough" woman who feared nothing and would even fight men who offended her. In this context, she charged that gone were the days when men used to be associated with power because of their physique. Instead, she said it was time for women to "shine," which I understood to mean transgressing femininity as socially constructed by local cultural frameworks. Above all, Zainabu emphasized that what was important for harmonious friendships among peers was "respect"; where those who offend would acknowledge it and then seek forgiveness. The term "respect" in this context embodies fear. Some speakers of Sheng appropriate the Swahili term tuheshimiane 
'let us respect each other', but use it to mean 'give me space'. Some of Zainabu's

claims above are captured in the following extract:

1. Zainabu: Sipendi mambo ya magrooups sana hapa chuo sababu pia unajua ukiwa shule ukianza anza magroups kitu kibaya kihappen, nyote mtakuwa in trouble. Waona? Sasa afadhali ukae solo ukae kivyako bora you know what you are doing, yah. So me I socialize with everyone but mostly the boys.

2. MW: Mostly the boys?

3. Zainabu: yah sababu either msichana mimi nitakupiga

4. MW: Utamweka war?

5. Zainabu: Ok. Mimi ni mtu as in, umeelewa mambo ya matomboys?

6. MW: Yes I know

7. Zainabu: Watu ambaye as in hawapendi mashow offs. Saa the best thing ni kuwaavoid maanake if you piss me off

8. MW: utamweka vita?

9. Zainabu: Si ati nitakuweka vita, nitakutandika na hutanifanyia lolote. Waona?

10. MW: Ehe! Wow

11. Zainabu: Me naweza kutandika. Hiyo si uongo. Mimi nitatandika. Hata jamaa pia mimi nitakutandika. Utanifanya?

12. MW: Wow, huwa huogopi?

13. Zainabu: There is nothing to fear.

14. MW: Eeh, but majamaa si wako na nguvu?

15. Zainabu: Who said?

16. MW: Wakionekana tu

17. Zainabu: Who said?

18. MW: Well, that is a good one

19. Zainabu: Watu wanasema majamaa wako na nguvu juu? Kwanzia kitambo, majamaa kila siku huwekwa mbele. Waona? But hii ni time saa hii pia madame tunafaa kushine pia sisi.

20. MW: Hapo ni kweli. You are right.

21. Zainabu: So hakuna cha kuogopa saa hii. Kama jamaa akijaribu kukutandika, tandika yeye. But provided there is one thing, respect. Kama kuna respect, hakutatokezea war.

22. MW: Hapo ni kweli. Watu wanaheshimiana

23. Zainabu: Mna heshima, mtu akikukasirisha anakuja anakuomba msamaha. Ukikasirisha mtu na ujue umemkasirisha mtu. Kama ni bahati mbaya, hapo labda. Lakini kama unajua umefanya intentionally, unaenda unaomba msamaha, things get back to normal. Unaona?

24. MW: Ni kweli.

25. Zainabu: So respect is the root to a happy environment pahali popote unaenda.

26. MW: Respect?
1. Zainabu: I don't like being in social groups in school because all of us would be punished in case something bad happens. You see? It is better you stay alone or be on your own provided that you know what you are doing, yah. So me I socialize with everyone but mostly the boys.

2. MW: Mostly the boys?

3. Zainabu: Yah because if it is a girl, I am very likely to beat/fight her

4. MW: You will beat her up?

5. Zainabu: Ok. I am a person who is like, do you understand tomboys' life?

6. MW: Yes I know

7. Zainabu: We do not like people who show off. So the best thing is to avoid them because if you offend me,

8. MW: You will fight him/her?

9. Zainabu: Not that I will fight you. I will beat you up and you won't do anything to me. You see?

10. MW: Ehe! Wow

11. Zainabu: I can beat you up. And I am not kidding! I can beat. Even a guy, I can beat. What will he do?

12. MW: Wow, don't you fear?

13. Zainabu: There is nothing to fear.

14. MW: Eeh, but aren't guys strong and tough?

15. Zainabu: Who said?

16. MW: Just looking at them

17. Zainabu: Who said?

18. MW: Well, that is a good one

19. Zainabu: Why do people say guys that guys are strong? Since time immemorial, guys have always been regarded as superior! This is the time we girls need to shine/rebel.

20. MW: That's true. You are right.

21. Zainabu: So, there is nothing about fearing here. If a guy tries beating you up, beat him up too. But if there is respect, there will be no war.

22. MW: That is true. People will respect each other.

23. Zainabu: If you respect each other; one who offends will come and seek forgiveness. If you offend someone accidentally, that is ok but if you realize it, you need to seek forgiveness and things will go back to normal. You see?

\section{MW: That is true.}

25. Zainabu: So respect is the root to a happy environment wherever you go.

26. MW: Respect? 
27. Zainabu: Respect.

27. Zainabu: Respect.

(Zainabu 04/2012, interview)

In Nakuru, I also found that there were female students who engaged in behaviours and sought identities that transgressed local conceptualizations of femininity. For example, I learned from my student informants that there were social groups which identified themselves with specific names like The Hot 7 'The Hot Seven', Action Boys, Earthquakes and Wazito. Some of them were single-gender while others were mixed. My informants told me that these social groups often engaged in activities that often put them in conflict with school authorities. As a result, they were not willing to be interviewed or observed, possibly because they feared that I would report them to the school authorities. However, I managed to gather useful information from my informants who knew about them, but I will limit my discussion to the social group called Wazito.

I asked students Hora and Bakari, introduced earlier, about their understanding of the meaning of the label Wazito as used among peers. They explained that Wazito was a group of five girls in different Form Two classes whose actions were always in conflict with school authorities. They spoke Sheng and engaged in social practices that "normal" girls did not, such as wearing short skirts, dating boys and going to discos during weekends. According to Hora and Bakari, these girls engaged in these social practices in order to be distinct from the rest. They adopted the name Wazito from the school rugby team, whose members described themselves as Wazito because they were big-bodied, and had remarkable success in inter-schools' competitions in rugby. 
However, school authorities were usually opposed to these social groups. For example, recall that Mrs. Kasisi and Mr. Nyachae's descriptionof the rugby team as "rough" boys, who spoke Sheng, used vulgar terms and had discipline problems, exemplifies this opposition to social groups by school authorities. Girls who identified themselves as Wazito thus transgressed culturally prescribed femininity by doing things not expected of "normal" girls. The various social groups are speakers' social categories of identity through which they define themselves. This argument is well supported by the sentiments about Wazito in the following extract:

\section{MW: So, Bakari umesema Wazito ni nani?}

2. Bakari: Wazito ni wasichana wa Form Two ambao wanafanya vitu zingine hazilingani na school rules.

3. MW: Eeh, ni wakora wakora ama ni rende tu ya, how would you describe them? How would you describe Wazito?

4. Bakari: Ni group, yaani ni rende ya watu; wasichana. Basically ni wasichana of about sijui kama ni watano. Huwa wana, maadili yao hayalingani na school rules. Eeh,

5. MW: Na tunaposema maadili yao hayalingani na school rules, tunamaanisha wanafanya mambo kama gani?

6. Bakari: School uniform ya wasichana; skirt inafaa kuwa below the knee. Hao wanafaa yenye ni above the knees. Eeh,

7. MW: Ooh. Na hili jina "Wazito" linaashiria tabia zao ama ni uzito wa?

8. Hora: Ni uphysically. Wote physically ni wahuge, wote [...]

9. Bakari: Eeh, wamekula [...] Aah hiyo jina inalingana na actions zao [...] Wanafanya vitu mzito zenye wasichana wa kawaida hawafanyi [...] Tuseme hawaharass directly. Yaani, wanataka tu kuwa recognized [...]

10. Hora: I think you can remember ile siku nilikuwa nimekaa pale kwa deputy [Yes, Mw]. $\mathrm{Ni}$ hao wote walikuwa wamekaa pale. You remember? Sasa walikuwa wamepigana, wamepigana among themselves; fighting for a boy. Sasa hizo ndizo vitu wanafanya [...]

11. MW: [...] Haya besides ukubwa, na venye
1. MW: So, Bakari who did you say are the Wazito?

2. Bakari: Wazito are girls in Form Two who engage in things that conflict with school rules.

3. MW: Eeh, are they crooks or is just a group of, how would you describe them? How would you describe Wazito?

4. Bakari: It is a group, i.e., it is a social group of people; girls. Basically, they are about, I am not sure whether they are five girls. They have values that conflict with school rules. Eeh,

5. MW: And when we say their values do not match school rules, what kind of things do they do?

6. Bakari: The prescribed school uniform for girls requires them to wear skirts whose length goes below the knees. However, Wazito wears short skirts whose length is above knees.

7. MW: Ooh. And does the name Wazito refer to their behaviors or what does it mean?

8. Hora: It's physique; all of them are huge [...

9. Bakari: Eeh, they have eaten ['huge'] [...] Aah, the name corresponds to their behaviours [...] They do "heavy" things that normal girls do not $[. .$.$] That is, they want to be recognized$ [...]

10. Hora: I think you can remember that day at the Deputy's ['office'] [Yes, MW]. All those who were there are the Wazito. Do you remember? They had fought each other because of a boy. Those are the kind of things they do. [...]

11. MW: [...] Ok. And besides physical size and 
ulisema wanavalia skati fupi which is against the school rules, what other observable behaviors?

12. Hora: Unajua hata outside the school, wanacarry hizo behaviours; sasa unawapata kwa Discos. Hizo characters kama hizo. Kama ni Saturday ikikuja, hizo ndizo vitendo huyu alikuwa anaongea. Saturday walikuja pale, Saturday. Wakakuja sasa kuchukuana pale wanaconnect sasa wanaenda may be Disco, may be dates kila weekend jioni. Hizo ndizo vitu zenye wanafanya.

13. Bakari: Ama unapata sasa kama saa hii wako na simu shuleni, mmh

14. Hora: Yaani ni watu wako crude. Yaani wako against school rules eeh [...]

15. MW: Na kuitwa waliitwa na wanafunzi wengine wazito ama?

16. Hora: Walijiita wenyewe.

17. Bakari: Walijiita. Hilo jina walitoa kwa Rugby. Rugby walijiita wazito juu it is a team ilitoka chini ikaenda ikigrow. Wanajiita wazito. Yaani wameanza kufanya remarkable changes kwa hii shule kwa huo mchezo wa rugby. the short skirts that you said is against school rules, what other observable behaviors?

12. Hora: You they carry on such behaviors even outside school. You will find them in Discos. Such characters. Like when its Saturday, those are the kind of things that Bakari was saying. The group met here another Saturday and left may be for Disco or for a weekend evening date. Those are the kind of things that they do.

13. Bakari: Or you will find like now they have phones in school, mmh.

14. Hora: These are crude people. That is, they are against school rules, eeh.

15. MW: And were they labeled Wazito by other students or?

16. Hora: They labeled themselves.

17. Bakari: They labeled themselves. They got that name from the rugby team. The rugby team called itself Wazito since it is a team that has come a long way and have started making remarkable changes ['impact'] to the status of this game in this school.

(Hora and Bakari, 10/2011, focus group--Nakuru)

These transgressive social groupings are not peculiar to Kenyan high

schools. They are social categories of identity also found in schools outside

Kenya. For example, Eckert (1989) conducted an ethnographic study in a high

school in the U.S., and found that students' friendship networks were

organized into two main social categories, Jocks and Burnouts, which were

comprised of both males and females. The two had opposing interests and

desired social identities. According to Eckert (1989: 74), “[t]he Jocks seek

independence from the family in intense involvement in adult-sanctioned peer

institutions, while the Burnouts seek it in a peer society that rejects the

authority of these institutions." In school settings, for example, Eckert found

that the Jocks were pro-school activities, hence teachers approved of them and

used them as points of reference with regard to discipline and academic 
performance. Teachers also rewarded them with good grades and leadership positions. Eckert elaborates that this preferential treatment of Jocks in academic settings played a significant role in the development of Jock networks and in the separation of Jocks from Burnouts. The Burnouts on the other hand had a counter-school ideology, engaging in rebellious social practices such as going out for dates, drinking alcohol, and smoking, which put them in conflict with school authorities. This difference in value systems between the Jocks and Burnouts created a form of antagonism between them, since the behavior of one threatened or exposed the basis of the other.

Male speakers of Sheng and lugha ya mtaa also challenge local conceptions of masculinity. For example, recall students Tinga and Mkinango from Mombasa reported that some male students modified their school uniform in order to look like celebrities such as Lil Wayne, who put on skinny trousers and sag them. Others like Tinga put on tightened shirt sleeves in order to emphasize their biceps. These modifications contravene socially acceptable modes of dressing for males both in school and in the wider society. In fact, various figures of authority such as parents and teachers regard them as markers of indecency. For example, I reported in Chapter Five about Mr. Nyachae who said that he did not tolerate students who sagged their trousers. Instead, he reprimanded them instantly and suspended those who continued with these deviant behaviors. Interestingly, some of these students like Tinga, who modified their school uniform, regarded these modifications as style or swag in some contexts but evaluated them negatively in others. So, how would 
one make sense of these opposing and sometimes overlapping social categories of identity employed by high school students?

Scholars such as Bucholtz (1999) and Eckert (1989) argue that the notion of community of practice is a useful analytical tool for understanding social categories such as the Jocks and Burnouts. For the purpose of this study, I employ the notion of community of practice to interpret how speakers like Zainabu and Tinga managed to negotiate multiple and sometimes contradictory social identities. Previous studies on Sheng such as Samper (2002) and Githinji (2006) have documented similar scenarios. Samper (2002: 202) attributes these contradictions to the fact that "Kenyan youth identity is not a stable, fixed state of being; it is constantly under revision." He argues that speakers mobilize these multiple identities for survival; some may be rebellious while others may be conformant. In this context, while nonspeakers of Sheng or outsiders might perceive the various identities as contradictions, speakers see or experience them as elements of the same "self"--that is, who they are and hope to become.

In summary, this chapter has examined the motivations behind young Kenyans' turn away from the officially sanctioned Standard Swahili and English in favor of the unauthorized Sheng and lugha ya mtaa. I have argued that there is a conflict in ideology between speakers of these nonstandard language varieties and school authorities on linguistic "correctness" and “decency." While school authorities advocate for uniformity, speakers of Sheng and lugha ya mtaa have embraced difference (from what is prescribed), which manifests itself in terms of style and fashion. In this sense, speakers of 
Sheng and lugha ya mtaa regard use of Standard Swahili and English as unfashionable, hence they ridicule those who speak the two languages. They also referred to them using pejorative labels such as Mtu wa English, show offs, wasomi for those who spoke English and washamba in the case of Standard Swahili. On the contrary, peers praise those who speak Sheng and lugha ya mtaa claiming that they have swag. These language attitudes suggest that use of Sheng and lugha ya mtaa has more social rewards among peers than either Standard Swahili or English. This partly explains why despite intensive efforts in schools to promote the officially sanctioned languages, the unauthorized Sheng and lugha ya mtaa continue to flourish.

However, while the embrace of Sheng and lugha ya mtaa suggests resistance against the "standard language ideology," speakers also engage in social practices that suggest conformity to the same. I argue that speakers operate in a "paradox of norms" (Kiessling and Mous 2004; Wairungu 2013) where they have to balance between associating with peers on the one hand and pleasing school authorities on the other. With regard to resistance, speakers employ Sheng and lugha ya mtaa to criticize and mock authority. Some speakers also dispute the common claim among educators that these nonstandard languages "corrupt" Standard Swahili and English. Instead, they argue that they know the appropriate languages to use in academic contexts and are able to switch back and forth when required to do so. To bolster this position, some students in the present study said that they knew of students who spoke Sheng and still scored excellent grades in exams. Another category of students reported that they employed Sheng productively during peer 
teaching and academic group discussions. They argued that Sheng enabled them to decipher concepts that they found difficult when framed in Standard Swahili or English. Some teachers echoed these sentiments, with some emphasizing that Sheng helped to reduce social distance between them and students. Others claimed that Sheng helped them accommodate students who may have serious social problems and have difficulties expressing themselves in English and Standard Swahili.

Speakers also challenged the "standard language ideology" by socially constructing "streets" and "ghetto" as sites of "smartness" rather than sites of "crime" and other gangster activities. Some of the speakers reported that "ghetto" had been their college of life. They argued that the knowledge of Sheng gained from "ghetto" would enable them to survive in any other environment as opposed to their counterparts in affluent neighborhoods associated with English, who they said would die if brought to live in "ghetto." Similarly, speakers of Sheng and lugha ya mtaa contravene the “standard language ideology" by regarding linguistic manipulations characteristic of the two language varieties as markers of ingenuousness and communicative efficiency rather than "errors." In addition, speakers of Sheng and lugha ya mtaa also contravene the formality aspect of the "standard language ideology" by employing vulgarities in their conversations, regarding them as normal and sometimes giving them positive meanings. In the process, these speakers transgress some of the culturally ascribed identities such as femininity and masculinity. Specifically, they contravene the common association of Sheng and lugha ya mtaa with male speakers and use of the 
standard and formal languages with female speakers. Besides language, male speakers on the one hand sag their pants and wear shirts exposing their biceps. Female speakers on the other handtrim and tighten their skirts to reveal body figures and these social practices challenge the culturally ascribed masculinity and femininity.

Though speakers of Sheng expressed negative attitudes toward the officially sanctioned languages, some still subscribed to the colonial language ideology which associates English but not Standard Swahili with intelligence or having gone through formal education. This difference is well captured in the use of the label wasomi 'scholars' to refer to those who spoke English while referring to those who spoke Standard Swahili as washamba 'dimwitted'. Other speakers subscribed to the myth of "non-accent," arguing that people go to school in order to replace their ethnic languages with English. Such language attitudes contribute to the hegemonic status that English has enjoyed over other languages in Kenya since the colonial period.

Another category of speakers subscribed to the "purist" ideology which regards nonstandard language varieties as "errors" or "pollutants" of the standard. A good example is student Zainabu who reported that speaking Standard Swahili was "healthier," while disparaging lugha ya mtaa as "full of trash.” In addition, there were speakers such as Tinga, who first claimed that Sheng and lugha ya mtaa were fashionable only to later claim that they were not. I attribute such conflicting language attitudes to the fact that speakers belonged to multiple social groups with opposing language ideology. 
From another point of view, the discussion demonstrated that both local and global media play a significant part in socializing youth about global styles, especially African American popular culture such as rap music. For instance, I drew comparisons between Sheng and lugha ya mtaa with other nonstandard languages such as Hip Hop Nation Language in the U.S. I argued that speakers embrace them as alternative cultural forms for negotiating desired freedom and distinct social identities, because they find those ascribed to them by the local cultural frameworks both limiting and undesirable.

Even though, I do not suggest that Kenyan youth are passive consumers of these foreign styles. Rather, they selectively interpret them and re-appropriate elements that suit their own styles and purposes. However, despite the desire to be distinct, speakers of Sheng and lugha ya mtaa still find themselves associating with the local cultural frameworks for survival. Similar observations were made by Kiessling and Mous (2004) who studied different urban youth vernaculars in post-colonial urban Africa including Sheng, indicating that these contradictory language attitudes are a common characteristic of urban youth vernaculars on a broader scale.

In conclusion, the antagonistic relationship between the dominant official language ideology on the one hand and speakers of Sheng and lugha ya mtaa on the other is rooted in their differences in the evaluation of language competence. While school authorities reward a speaker's ability to use the standard, speakers of Sheng and lugha ya mtaa reward the ability to innovate from the various languages in a speaker's repertoire. I also conclude that social factors such as a speaker's identity play a significant role in his/her 
language choices and attitudes. However, these social identities are not static but fluid and contextual. In this regard, it is important for school authorities to recognize that not all students speak Sheng and lugha ya mtaa. Moreover, not all speakers use the two language varieties for the "wrong" reasons. Some students and even teachers use them as learning resources. School authorities should therefore adopt a different approach for addressing the presence of Sheng and lugha ya mtaa in schools since continued efforts to eradicate them are counterproductive. In the next chapter, I discuss the paradoxical notion of "shrubbing," which speakers of Sheng and lugha ya mtaa employed as a counter-strategy against the "standard language ideology," and local cultural frameworks. 


\title{
Chapter Seven
}

\section{0. "Bringing Them Down": "Shrubbing” Discourse among Young Kenyans}

\begin{abstract}
Obviously, you cannot use mother tongue to seduce a girl. I hope you understand what I am saying! The point is that when you mix languages, girls see you as trendy. Guys don't like girls who speak English with a mother tongue influence. Guys despise such girls. They look down upon them. For instance, such a girl may have problems pronouncing /r/ and /l/ and her shrubbing might embarrass you if you took her out in the company of your friends. For me, though I may love a girl who shrubs, I cannot converse with her in the presence of my friends. That is why we said earlier that Sheng helps in such situations. Like for my case, I have problems with $/ \mathrm{r} /$ and $/ 1 /$. So, if I want to say kulala, I will say kutuna. That way, Sheng helps me escape such embarrassing situations. (Munne, Form Four focus group 4, 11/2011--Nakuru, my translation)
\end{abstract}

The popularity of nonstandard language varieties such as Sheng and lugha ya mtaa among young Kenyans over the officially sanctioned English and Standard Swahili is indisputable. So, while embracing these nonstandard language varieties, young Kenyans have innovated a counterstrategy that they call "shrubbing" or kushema/kuponyoa/kung'oa/kunyoa/kuangusha, ${ }^{79}$ to resist the dominant "standard language ideology" that associates social rewards with speaking the officially sanctioned languages. Young Kenyans describe "shrubbing" as speaking either Standard Swahili or English with an ethnic/mother tongue accent, and also adding or dropping sounds "inappropriately." It also happens when a speaker confuses two closely-related sounds or vocabulary items containing these sounds (minimal pairs), hence affecting the intended meaning.

Shrubbing is stigmatized: peers expect those who speak the two officially sanctioned languages to do so without "errors." Speakers claim that when they engage in the "shrubbing discourse," they do so to "bring down" enthusiasts of the officially

\footnotetext{
${ }^{79}$ The $k u$ - prefix indicates that the verb is in infinitive form (Hinnebusch and Mirza 1998). Shema is the "shrubbed" form of the Swahili word sema 'say'. Nyoa is Swahili for 'shave', ng'oa Swahili for 'remove/uproot', angusha is Swahili for 'drop' and ponyoa is a coined term in Sheng meaning 'shrub' in the Kenyan sense.
} 
sanctioned Standard Swahili and English. (I use the term "shrubbing discourse" to refer to the act of pointing out or highlighting that a speaker has shrubbed.)

However, a critical look at the various social practices involved in shrubbing discourse suggests both resistance and compliance to the dominant official language ideology. So, what social meanings are associated with the shrubbing discourse and how are these meanings related to speakers' desired identities? Is the discourse limited to the use of Standard Swahili and English, or does it also apply to nonstandard forms of the two languages? Owing to the fact that Sheng draws its linguistic material from Swahili, English and ethnic languages, does shrubbing occur in Sheng? What about lugha ya mtaa in the case of Mombasa? If it does, do speakers perceive it differently from when it occurs in other languages? I engage these questions in the remainder of this chapter by exploring notions of shrubbing and mother tongue interference, semiotics of the shrubbing discourse, conformity, resistance and the ingeniousness in the shrubbing discourse. Finally, I discuss shrubbing discourse in Nakuru and Mombasa, and how shrubbing as a performance generates symbolic capital among speakers.

\subsubsection{The notion of shrubbing vs. Mother tongue interference: a question of generation}

According to Mr. Fujo, the teacher from Mombasa mentioned in the previous chapter, what young Kenyans call shrubbing today has been in existence among older generations for many years, but going by a different name, "mother tongue interference" (Mr. Fujo 01/2012, interview) . Mrs. Wanjala, the teacher from Nakuru opined that native speakers of every ethnic language in Kenya have particular sounds 
in Standard Swahili and English that they find challenging in pronunciation, and sometimes when writing (Mrs. Wanjala 11/2011, interview). She observed that many native speakers of Kikuyu have difficulties distinguishing between $/ 1 /$ and $/ r /$ in their pronunciation. Also, she said that native speakers of Dholuo do not distinguish between $/ J /$ and $/ \mathrm{s} /$, resulting into the common pronunciation of the word fish, for instance, as [fis]. She noted that members of her ethnic community, Luhya, had problems pronouncing $/ \mathrm{p} /$ and $/ \mathrm{b} /$. The latter admission is an indication that even members of given ethnic groups are aware of these ethnically marked pronunciations. $^{80}$

Many Kenyans including speakers from the same ethnic background, use these linguistic challenges as stereotypes to parody or ridicule others'ethnic identity. It is also common for Kenyans to use words from ethnic languages in an exaggerated or joking manner as ice-breakers when initiating a conversation during interethnic social interactions. For instance, in the early days of my research in Nakuru, some teachers who were non-Kikuyus would utter Kikuyu words, exaggerating the pronunciation of shibboleths in order to draw my attention and engage in a conversation. A good example is the Kikuyu expression atĩrĩrĩ [at'ĩñ̃ĩ] 'let me tell you', commonly used as an attention grabber when introducing what someone is about to say. Non-Kikuyu Kenyans often use this expression with Kikuyu interlocutors, but pronounce it with an exaggerated trilled [r], which highlights the stereotype that Kikuyu speakers use [r] when [1] is expected. Unfortunately, such shibboleths, as mentioned in Chapter One, are often used to identify members of the targeted ethnic groups during interethnic clashes, especially in the period surrounding national elections.

\footnotetext{
${ }^{80}$ Mrs. Wanjala's observation is based on actual differences between the phonemes of these languages and those of English and Swahili.
} 
I also witnessed situations where both native speakers of Kikuyu and nonKikuyu would make fun of my ethnicity and openly claim that it was easy for them to tell that I was Kikuyu by just listening to how I articulated /l/ and /r/, and /t $\mathrm{J} /$ and $/ \mathrm{f} /$. Such claims are sometimes informed by the colonial ideology discussed in Chapter Four, which associates each "ethnic language" in Africa with one "tribe" (Gal and Irvine 1995; Hymes 1968). Most recently, it has become common for Kenyans to email one another or send short text messages containing different forms of ethnic mockery, as in the extract copied below. The extract is from an e-mail that I received from a close friend I will call Muremu who lives in the United States. Muremu is not Kikuyu but she knows that I am Kikuyu and from Othaya in Central Kenya. The language used in the extract depicts the writer as writing Swahili with a strong Kikuyu influence, marked by conspicuous use of /r/ in place of /1/ and adding and dropping sounds "inappropriately." The practice of circulating such e-mails suggests that participants subscribe to the ideology that seeks to erase internal or individual variation among speakers of ethnic languages such as Kikuyu. For demonstration purposes, I use square brackets for the sounds that have been dropped, bold for the sounds that have been added, and underline for the $/ \mathrm{r} /$ that has been pronounced in place of /1/ (the basis of comparison is Standard Swahili).

THIS NOTICE was spotted at KARUMAINDO BAR in Othaya.

1. Ukifanya order, unaturia, usishi[n]de umeku[m]busha waiter, yeye ni mutu mzima anaku[m]buka.

2. Ukirewa urewe peke yako, usianze kui[m]ba nyi[m]bo za Kigooco huku. Ukitaka kui[m]ba e[n]da crusade.

3. Huku hakuna dance floor. Kwa hivo usikadance huku, hii ni bar, sio carnivorous(Carnivore). ${ }^{81}$

4. Urinal ziko. Kukonjoa ni sawa. Ukitaka hanja kubwa, mariza po[m]be yako ue[n]de ukakumie kwako. Huku hakuna choo. Tunauzaga fo[m]be si Ugari.

(Mигети 07/2012, E-mail)

\footnotetext{
${ }^{81}$ The original Carnivore is a big restaurant known for selling game meat and open-air entertainment. It receives tourists from different parts of the world and has recently become popular among many young Kenyans because it hosts stand-up comedies such as Churchill-Live.
} 
THIS NOTICE was spotted at KARUMAINDO BAR in Othaya.

1. Once you place your order, do not keep on reminding the waiter. $S /$ he is an adult with good memory.

2. If you get drunk, get drunk alone. Do not start singing songs of praise and worship here; if you want to sing, go to a crusade.

3. Here there is no dance foow. So do not dance here, this is a bar not the social joint called Carnivore.

3. We have urinals. So, short calls are ok. But for long calls, finish your beer and go home to poop. Here there are not toilets; we sell beer not corn meal/food.

(Muremu 07/2012, E-mail)

Owing to the fact that the immediate former president of Kenya was from Othaya and that speakers associate mother tongue interference with communicative inefficiency, I inferred that Muremu meant to make fun of both my ethnic and local identity, an inference that is also supported by the subsequent conversations I had with her. Also, it is possible that Muremu sent me the e-mail to signal our mutual friendship, because I would have otherwise been offended if she were not my friend, because the content of the email and the tone of the language are socially inappropriate. This is similar to the speakers of Sheng and lugha ya mtaa who used abusive language to one another to express solidarity and close friendship.

The various examples in the foregoing discussion exemplify the earlier claim by Mr. Fujo, that talk about mother tongue interference as a discursive practice has been in existence among the older generations for many decades in Kenya. However, my research findings indicate that the current generation is more invested in highlighting or drawing attention to the practice (i.e., engaging in the shrubbing discourse). For instance, while the older generations let speakers to easily get away with mother tongue interference, the current generation reprimands shrubbers instantly. Also, since shrubbing is a major concern among young Kenyans, they sometimes draw adults into shrubbing discourse even if these adults would have otherwise let it go. For example, a close friend called Mwandani who participates in 
such discourse narrated to me how her family had gone for two weeks without electric power in 2010, and they all got excited when the power was finally restored. She and her brother turned on the lights both within and outside their house, making their home look like a small city. They invited their mother to step out of the house and see her fully lit home. She exclaimed, "Uuuw, this is Nalobi city!" Interestingly, Mwandani's mother did not notice that she had shrubbed /1/ instead of /r/, and even repeated these words after she was prompted by Mwandani. However, Mwandani and her brother had noted that their mother had shrubbed and pointed it out right away. They also laughed sarcastically at her, drawing the attention of their dad who stepped out of the house to share the fun. He then started teasing his wife asking her to trill her tongue--"rrrrrrrr"--several times, in the guise that this would help her avoid shrubbing in the future.

Mwandani told me that even though her mother did not trill as asked by the dad, the incident created a happy mood and this is why it was still fresh in her mind (Mwandani 07/2013, casual conversation). The fact that Mwandani recalled this particular incident after such a long time confirms that shrubbing is a major concern among young Kenyans, and that they are more invested in it than the older generations. Also, the current generation's notion of shrubbing is more conventionalized and expresses additional social meanings. Some of these meanings suggest compliance with the sociocultural confines of the local cultural frameworks and the dominant official language ideology while others indicate resistance to the same. So, what are these meanings and in what ways does the shrubbing discourse express conformity and resistance? 


\subsubsection{Semiotics of the shrubbing discourse}

As mentioned earlier, I use the term shrubbing discourse to refer to the act of pointing out or highlighting that a speaker has shrubbed common among young Kenyans. Many speakers reported that they engage in the shrubbing discourse for fun, but the way that fun is generated is contextual, and elicits different reactions from the shrubbers. In one context, peers make fun of the shrubber and laugh at him/her. They, however, do not expect the shrubber to take offense but instead enjoy and get the fun of it. Even though participants who take offense usually lose their face (Goffman 1959), peers expect them to overcome their anger in a short time. Therefore, peers regard participants who continue showing their anger negatively, especially if these participants threaten to get physical when peers make fun of them for shrubbing. For example, I asked students Hora and Bakari from Nakuru to comment on my observation that peers often nicknamed those who shrubbed. They responded in the affirmative but cautioned that not all peers take it positively. This is well captured in the following extract contrasting two of their friends who shrub, but who responded to peers' reactions differently:

1. MW: Na kuna mtu aliniambia shrubbing, ati shrubbing...

2. Bakari: Eeh inakupatia nickname

3. MW: Inakupatia nickname?

4. Hora: Sasa ndio tunakwambia kama case ya ule jamaa ule Ngengi, uule Ngengi. Sasa ukimpatia jina inatokana na hapo mnaweza, hata anaweza kukupiga.

5. MW: Aaah anakasirika?

6. Hora: Eeeh, anakasirika sana. Anaona kama unamcheka ni mshamba.

7. Bakari: Kuna wa darasa yetu lakini yeye anachukulia, anajua ni jokes. Anaitwa Boinem. Yah, yeye ni Mkalee. Yah, sasa huyo hata akishrub,

8. Hora: Anacheka tu na mnaendelea.
1. MW: And there is somebody who told me that shrubbing, that shrubbing...

2. Bakari: Eeh, it earns people nicknames

3. MW: Can earn you a nickname?

4. Hora: Now, like we were telling you about that guy called Ngengi. If you nickname him for shrubbing, he can even get physical with you.

5. MW: Aaah he gets angry?

6. Hora: Eeeh, he gets very angry. He thinks you are laughing at him because he is "country."

7. Bakari: There is a guy from our class, he takes it easy. He knows it is jokes. He is called Boinem and is Kalenjin. Even if he shrubs

8. Hora: He just laughs and moves on. 
9. Bakari: Aah! Anacheka tu na anajua ni jokes. Kama kuna siku tulikuwa tunasoma Kifo Kisimani wakasema pahali, kuna mtu alikuwa anasema 'Bafe Bafe, Tazama Bafe'. Naye akasema 'Pafe Pafe, Tasama Pafe'. Watu sasa wakaenjoy. Yeye hachukulii seriously. Na bado maisha inaendelea. Watu sasa wanazoea tu. Anajua ni jokes tu. Hachukulii serious.
9. Bakari: Aah! He just laughs and understands that it is jokes. Like there is a time we were reading an assigned book called Kifo Kisimani and one of the characters said 'Bafe Bafe, Tazama Bafe'. But Boinem read, 'Pafe Pafe, Tasama Pafe'. People in our class enjoyed [it] and Boinem did not take offence but instead moved on. So people have known him like that. $\mathrm{He}$ understands that it is jokes, hence he never takes it seriously.

(Hora and Bakari, 10/2011, focus group--Nakuru)

But why do speakers respond differently when peers point out that they have shrubbed? According to Lippi-Green (2012: 45), even though accent generally means an individual's way of speaking, “in the serious study of accent, the object is not what comes out of one person's mouth, but what the listeners hear and understand." Similar observations have been made by other scholars such as Derwing and Munro (2009: 478) who assert that, '[f]rom our perspective, listeners' judgments are the only meaningful window into accentedness and comprehensibility.” In this regard, participants in shrubbing discourse make different meanings from speakers' accents. Some of these meanings are emotionally provocative, especially if those parodied feel like the performance is reifying their subordinate or disadvantaged social status. As a result, not all speakers approve of ethnic parodying; some exhibit resistance while others submit in order to satisfy their desire to belong to a social group. These are the reactions exhibited by students Ngengi and Boinem respectively in the extract above.

Other scholars who have studied ethnic mockery as performance have made similar observations. For example, Thomson (2010: 510) who examined ethnic parody in the song "I am Maasai" by Mr. Ebbo from Tanzania, observes that while Mr. Ebbo's ethnic parodying of the Maasai enables him to critique the hegemonic 
attitudes toward Standard Swahili in relation to ethnic languages, "[His] performance of Maasai identity remains uncomfortably close to local stereotypes of the Maasai and may therefore lend support to an ideology that critiques those Tanzanians who refuse to abandon their ethnicity in favor of postethnic version of modernity." Similarly Hill (1998), who studied the semiotics of Mock Spanish in the U.S. reports that though white speakers of Mock Spanish deny that their use of the language is racist, many Spanish speakers do hear the racist message of Mock Spanish. She gives supporting examples from two of her informants as follows:

In an interview, a Spanish-speaking Chicano high school counselor in Tucson said 'You know, I've noticed that most of the teachers never use any Spanish around here unless it's something negative.' A Spanish-speaking businesswoman said, 'when you first hear that stuff, you think, that's nice, they're trying, but when you hear more and more and you realize that there's something nasty underneath.'(Hill 1998: 684)

From another point of view, some speakers engage in the shrubbing discourse as a "style" (Irvine 1989) or performance. They generate humor by deliberately shrubbing stereotypical features either associated with their own ethnic communities or with others, expecting that peers would get the joke and laugh. Sometimes, shrubbing as a performance entails selective and ingenious stylization of stereotypic linguistic markers associated with targeted peers in ways that socially construct them as the "Other." "Othering" happens when the shrubber performs a stereotypical linguistic feature that elevates himself/herself socially over the targeted audience. In order for the audience to appreciate the embedded humor in "Othering," there has to be a shared context. For example, in a conversation with two students called Mwelusi and Andua in Nakuru, I asked them to comment about their understanding of the term shrubbing and why young people found it interesting. Mwelusi responded that: 
Mmmh, unaona hiyo saa hizi unakuta pengine kuna mtu, yaani imekuwa ni kama a form of jokes hivi. Juu pengine unaweza kuta kaa saa tunakaa kwa vikundi hivi, unakuta pengine mmeongea pengine hata ni kwa groupwork hivi unakuta mmeongea mmediscuss na hakuna mtu, yaani hakuna kitu yeyote imesemwa ya kuchekesha. Sasa unakuta mtu atakam na hako kaidea. Unakuta yaani ako na for example unakuta kama ni mtu wa kutoka huko Central, twajua vitu zenye zinasumbua ni hizo l na $r$. Sasa unakuta amekuja ameshrub sasa, but sasa amekosea $t$ na $d$. That means mnaona hii si ati ni ya kutoka huko ndani, apana, hii ameileta tu ndio atoe jokes tu nayo. (Mwelusi and Andua 10/2011, focus group--Nakuru)

Mmmh, it is like it has become a source of jokes and fun. You will find that, may be, you are in a group discussion and nothing has been said to make people laugh. That way, you will notice that somebody would come up with an idea to make peers laugh. For example, if a guy is from Central Kenya where people have difficulties pronouncing / $/$ / and $/ \mathrm{r}$ /, he may choose to make peers laugh by deliberately shrubbing stereotypical sounds not associated with people from Central Kenya, e.g., /t/ and /d/ [some of which may be linked to some peers in the discussion group]. (Mwelusi and Andua 10/ 2011, focus group--Nakuru)

Even though the examples given by Hora, Bakari and Mwelusi above

primarily focus on how the shrubbing discourse generates fun among peers, these examples also indicate that the discourse provides peers with an avenue for appreciating their ethnic diversity. This claim is also supported by the responses I got from some teachers such as Mr. Naibu from Mombasa, who reported that students usually got excited when he drew material from ethnic languages when teaching. This is captured in the extract from our interview below:

MW: Are there times during your lessons like when you are teaching History that you use other languages besides English?

Mr. Naibu: Sometimes I have to use other languages. Also, I ask the students the communities they come from, not because I am a tribalist but because I want us to learn from each other. For instance, we may talk about how sounds in a language are coming out, e.g., the words in Kikuyu come out differently from that of Kijaluo. So, in History, we talk about communities, their languages and their cultures. So, officially we communicate in English but, I can mention some other words in Kiswahili, in Kikuyu etc., if I want to emphasize a point.

MW: How do students respond when they hear you speaking or uttering words from other languages?

Mr. Naibu: The lesson gets very lively and they laugh throughout. Like the other day, I talked about how a Kikuyu would say "I love you" and I wrote on the board "I Rove You," and they were very excited. (Mr. Naibu 01/2012, interview) 
From another point of view, some of my field notes indicate that the shrubbing discourse is also a strategy through which speakers express disdain for ethnic languages. They perceive these languages negatively and regard them as markers of backwardness. For example, Kadogo, the student from Nakuru introduced earlier, reported that speakers avoided shrubbing because it was a sign of "primitivity" and "country." She noted:

Unapokuwa eloquent inakupa a higher status kuliko yule kila wakati anashrub kwa vile ataonekana primitive/mshamba. (Kadogo 07/2013, Email)

Being eloquent accords you a higher status than a speaker who shrubs all the time, since he appears primitive or country. (Kadogo 07/2013, Email)

Similarly, Andua, the student from Nakuru, reported that shrubbing marked the speaker as "old-fashioned," a term which has a connotation of lagging behind from a temporal point of view. She said:

1. Andua: [Laughs], mimi kulingana na mimi, [laughs], mtu akishrub, si kupenda kwake. Mostly unapata ni athari za lugha ya mama. Saa, [laughs], siku hizi imekuwa ni as if ukishrub, ni yaani wee uko sijui niite aje; it's like you are old, wewe ni old-fashioned.

\section{MW: Unachukuliwa wewe ni old fashioned?}

3. Andua: Eeeh kitu kaa hiyo, yah! Wewe ni mtu wa mashamba. So it becomes funny. It's as if sasa people don't expect you to shrub, so ukishrub inakuwa another issue. Itakuwa ni kaa uta, may be, utakuwa identified uniquely just because unashrub.
1. Andua: [Laughs], According to me, when a person shrubs, it is not his/her wish. Mostly, you will find that it is mother tongue influence. So, [laughs], nowadays it is like if you shrub it is like you are, I don't know what to call it! It is like you are old; you are old fashioned.

2. MW: You are perceived as oldfashioned?

3. Andua: Yes, something like that, yah. You are "country." That way, it becomes funny. It is as if people do not expect you to shrub, so if you shrub it becomes an issue. Peers will look at you differently just because you shrub.

(Mwelusi and Andua 10/ 2011, focus group--Nakuru)

Students Bakari and Hora from Nakuru expressed similar sentiments but their stance about shrubbing was different from Andua's. While Andua seemed sympathetic to speakers and did not blame them for shrubbing, Bakari and 
Hora faulted them, arguing that peers did not expect people of their generation

to shrub especially if one lived in the urban areas. In their words:

1. Hora: Unajua inaonyesha wewe ni mshamba.

2. Bakari: Yaani ama wewe ni wa town lakini tena aah [tsh!] umekuwa affected na mother tongue. Unajua sasa tunaexpect watu wa ii age yetu hii, hiyo haifai kuwa issue. Eti background ya kuongea, yaani mother tongue, eeh.

1. Hora: You know it shows that you are "country."

2. Bakari: Or maybe you are from town but now, [tsh!], you have mother tongue influence. You know we do not expect people in our generation to have mother tongue influence when they speak, eeh.

(Hora and Bakari 10/2011, focus group--Nakuru)

Contrary to Bakari and Hora, Mandumo, a student from Nakuru

admitted that even urban peers shrubbed (Mandumo 03/2013, Facebook

interview). She, however, claimed that these urban peers responded to

shrubbing differently as compared to their rural counterparts. That is, while

urban peers corrected themselves as soon as they realized that they had

shrubbed, their rural counterparts shrubbed throughout since they were not aware about shrubbing in the first place. Interestingly, despite their

conspicuous shrubbing, Mandumo said that these rural peers usually thought that they spoke Swahili or English "correctly." She therefore claimed that it was easy to distinguish between rural peers and their urban counterparts in social interactions, an assertion well captured in our conversation transcribed below:

1. MW: So, tell me why youth are conscious about shrubbing

2. Mandumo: When one shrubs, everybody makes fun of you, mpaka to an extend unaeza nicknamiwa jina yenye umeshrub,

3. MW: Eeehe! Endelea those are the stuff about shrubbing I want to know
1. MW: So, tell me why youth are conscious about shrubbing

2. Mandumo: When one shrubs, everybody makes fun of him/her to an extent that one can be nicknamed from what he/she has shrubbed.

3. MW: Eeehe! Go on. Those are the stuff about shrubbing that I want to know 
4. Mandumo: U know most guys fear to be known as born ushagos, thats why they fear kushrub ndio waseme they are born taos, wasionekane kama mafala, as in dunder heads.

5. MW: Oooh! So ukiambiwa unashrub means: 1) wewe ni mtu wa ushago ama Una ushamba; and 2) wewe ni fala/dunderheads.

6. Mandumo: Exactly my point! Even born taos at times ulimi unaeza teleza but $u$ make sure umerudia correctly, though lazima watu waku enjoy pia.
4. Mandumo: You know many young Kenyans do not want peers to know that they were born in the countryside. That is why they are that worried about shrubbing, hence they take serious caution so that they can easily associate with urban life and also for peers not to perceive them as dim-witted or idiotic.

5. MW: Oooh! So when you are told that you shrub, it means that: 1) you are "country"; and 2) not smart?

6. Mandumo: Exactly my point. Even those born in towns can sometimes shrub but one makes sure that $\mathrm{s} / \mathrm{he}$ has repeated the correct way even though peers will tease you.

(Mandumo 03/2013, Facebook interview)

The various parallels that students Bakari, Hora and Mandumo drew between urban peers and their rural counterparts suggest that the shrubbing discourse enables participants to show awareness of a desire to transcend ethnicity. For example, in the conversation above, Mandumo claimed that many young Kenyans did not want peers to know that they were born in the countryside. As such, she said that these speakers made every effort to avoid shrubbing, which would make them appear country and dim-witted among peers. In Chapter Four, I reported similar observations made by Mr. Naibu from Mombasa, who said that children nowadays were not as attached to their ethnic communities as it was the case during his time. He said that these children deliberately avoided associating with their ethnic backgrounds, suggesting that their level of identity was changing towards being more patriotic to Kenya than to their ethnic communities (Mr. Naibu 01/2012, interview). In this context, I argue that ethnic identity may play less of a role for the young Kenyans than for the older generations. Further, Mr. Mpoa from Nakuru reported that students spoke an informal variety of Swahili in peer interactions, hence they were more nationalistic than their teachers who often used ethnic languages. These examples articulate well with the 
claim by Kiessling and Mous (2004) that speakers of urban youth vernaculars in Africa transcend ethnicity by embracing urban life while dissociating from ethnic languages and rural life.

However, Kiessling and Mous (2004) argue that speakers of these vernaculars operate within a "paradox of norms." In this regard, while it is true that the shrubbing discourse enables young Kenyans to demonstrate a desire to transcend ethnicity, it also arouses their ethnic consciousness. For example, Kadogo, the student from Nakuru, confessed that students had a desire to appear perfect in English and easily dealt with grammatical mistakes whenever they appeared in their speech. However, Kadogo said that "it is 'shrubbing' that brings them down terribly because it reminds them of where they come from, that is their ethnic background" (Praise and Worship focus group, 11/2011--Nakuru). Kadogo's sentiments suggest that participants in the shrubbing discourse also conform to the dominant "standard language ideology," which disparages ethnic languages in favor of the standard. In the following section, I examine the various aspects of conformity that speakers exhibit in the shrubbing discourse.

\subsubsection{Conformity in the shrubbing discourse}

According to Lippi-Green (2012: 47), "in spite of all the hard evidence that all languages change, people steadfastly believe that a homogeneous, standardized, one size-fits-all language is not only desirable, it is truly a possibility." In this context, one of the major "errors" that teachers in Kenyan schools correct in students' exams is mother tongue interference in their spoken and written Standard Swahili and English. In our conversation with the 
student Kadogo, she reported that peers engaged in shrubbing discourse because it helped them correct one another when they made grammatical mistakes (Praise and Worship focus group, 11/2011--Nakuru). However, while it is true that mother tongue interference may lead to grammatical mistakes, I argue that what is important in the context of shrubbing is that Sheng enthusiasts such as Kadogo perceive linguistic variation as "errors." This further suggests that young Kenyans' obsession with linguistic "correctness" in the shrubbing discourse is a marker of conformity to the confines of the “standard language ideology" (Lippi-Green 2012).

From another point of view, participants in the shrubbing discourse perceive the various languages in their repertoire in a hierarchical manner, where they rank ethnic languages lowest. For example, recall that student Kadogo reported that speakers avoided shrubbing because it was a marker of "primitivity," while eloquence-equated with pronunciation that is devoid of ethnic marking--socially elevated the speaker. This contrast suggests that these speakers subscribe to the colonial/racist language ideology which disparages African languages in favor of European counterparts.

I analyze a few more indicators of conformity in shrubbing discourse here below, drawing from my conversation with student Mandumo. Even though Mandumo did not answer the question I had asked her, i.e, specifically whether it is possible for a Sheng speaker to shrub, her response is relevant because it reveals that she conforms to some aspects of the "standard language ideology" (line 1 of this conversation was used in an earlier extract): 
1. Exactly my point, even born taos at times ulimi unaeza teleza but u make sure umerudia correctly, though lazima watu waku enjoy pia.

2. MW: Ooh! Watu wenye huongea Sheng huwa wanashrub?

3. Mandumo: yeah,ata news anchors, na ndio nimesema [urban peers] wakishrub wanarudia the same word vile inafaa, but then again wasee wa ushago wakishrub hawajuangi coz they don't know kama kuna kitu inaitwa kushrub.

4. MW: Woow! So, kushrub ni kitu ya wasee wa tau?

5. Mandumo: yeah. it's very easy to spot msee wa ocha, eg, kiuks, shingo = chingo, kuchelewa $=$ kushelewa, chai $=$ shai, baraka $=$ balaka, etc

6. MW: Woow! Na watu wa tau wakikam shagz huambiwa wanashrub?

7. Mandumo: Watu wa tao huongea clean swahili, wasee wa ocha they dont know kushrub ni nini. Lemmi give u an example ya wasee wa ocha, "Nimeshelewa kasi kwa sabambu mathlee sime stlike." That person anajua ameongea vipoa but ameng'oa hiyo sentence yote.

8. MW: Na isipokuwa kuonekana mshamba na fala, what else do people say abt shrubbers?

9. Mandumo: nothing much, ni kuonekana tu ur not used na maisha ya town.
1. Exactly my point, even those born in towns can sometimes shrub but one makes sure that $\mathrm{s} / \mathrm{he}$ has repeated the correct way even though peers will tease you.

2. MW: Ooh! Do Sheng speakers shrub?

3. Mandumo: Yes. Even news anchors. And that is why I have said urban peers correct themselves once they shrub by repeating the same word correctly. But for the rural counterparts, they never realize when they shrub because they are not aware of shrubbing in the first place.

4. MW: Woow! So, shrubbing discourse is a thing of the urban peers?

5. Mandumo: Yes. It is very easy to identify a guy from the countryside, e.g., Kikuyus pronounce shingo as chingo; Kuchelewa as Kushelewa; Chai as Shai; Baraka as Balaka etc.

6. MW: Woow! And are urban peers told that they are shrubbing once they come to the countryside?

7. Mandumo: People from town speak clean Swahili. Rural peers do not know what shrubbing is. Lemme give you an example of how people from the countryside speak, "Nimeshelewa kasi kwa sabambu mathlee sime stlike." 'I am late because matatus are on strike.' That person thinks that he has spoken correctly but he has shrubbed the whole of that sentence.

8. MW: And besides appearing "country," naïve and "foolish/idiot," what else do people say about shrubbers?

9. Mandumo: Nothing much. Just being perceived as somebody who is not used to town life.

(Mandumo 03/2013, Facebook interview)

Mandumo's claim that even news anchors shrub is an indicator that she

regarded them as speakers of "good" language. This is an effect of the

"standard language ideology," which regards newscasters in the mainstream

media and grammarians as yardsticks for evaluating the speech of other

speakers and also as custodians of the "standard." Similarly, Mandumo's other

claim that "people from town speak clean Swahili" with the implication that 
they can speak Swahili without mother tongue influence, suggests that participants of the shrubbing discourse subscribe to the myth of non-accent, which is an effect of the "standard language ideology" (Lippi-Green 2012). In addition, Mandumo's other claim that many young Kenyans took every precaution to avoid shrubbing since it would mark them as "mafala, as in dunder heads," suggests that participants of the shrubbing discourse subscribe to the dominant "standard language ideology" which associates ethnic languages with lack of formal education and even lack of intelligence.

As we saw in Chapter Five, teachers in both Nakuru and Mombasa associated students' use of ethnic languages with "noise," lack of “civilization" and discipline problems. These teachers, however, approved of students who embraced English, arguing that such students were intelligent, courteous, respectful and academically ambitious. These teachers also told me that students who always spoke English came from the affluent neighborhoods, while those who spoke nonstandard language varieties such as Sheng came from the low-income areas. All these are effects of the "standard language ideology" which associates standard language with everything good while disparaging other language varieties.

Participants of the shrubbing discourse also subscribe to the colonial ideology which associates each local language with one ethnic group. For example, several students including Mandumo claimed that it was easy to tell peoples' ethnic identity using linguistic markers in their spoken language. Mandumo in particular gave the lexical items shown in Table (1) below as examples: 


\begin{tabular}{|c|c|c|}
\hline \multicolumn{3}{|c|}{ Stereotypic Linguistic Markers For Various Ethnic Groups in Kenya } \\
\hline Ethnic community & "Correct" Expected & 'Shrubbed' \\
\hline Kiuks (Kikuyu) & $\begin{array}{l}\text { Shingo } \\
\text { Kuchelewa } \\
\text { Chai } \\
\text { Baraka }\end{array}$ & $\begin{array}{l}\text { Chingo, } \\
\text { Kushelewa, } \\
\text { Shai, } \\
\text { Balaka }\end{array}$ \\
\hline Kamba & $\begin{array}{l}\text { Balozi } \\
\text { Eunice } \\
\text { Happy } \\
\text { Zero }\end{array}$ & $\begin{array}{l}\text { Balonzi, } \\
\text { Junice, } \\
\text { Appy, } \\
\text { Nzero }\end{array}$ \\
\hline Luo & $\begin{array}{l}\text { Fish } \\
\text { Samaki }\end{array}$ & $\begin{array}{l}\text { Fis } \\
\text { Smaki }\end{array}$ \\
\hline Kalenjin & $\begin{array}{l}\text { Pretty } \\
\text { Beautiful }\end{array}$ & $\begin{array}{l}\text { Bretty, } \\
\text { Peautiful }\end{array}$ \\
\hline Kisiis & $\begin{array}{l}\text { Sounds like Kales } \\
\text { ['Kalenjin'] }\end{array}$ & \\
\hline
\end{tabular}

Despite the fact that Kisii and Kalenjin have different phonologies, hence speakers of the two languages sound different in actuality; Mandumo's generalization seeks to ignore these differences. Her claim in the last row that "Kisiis sounds like Kales 'Kalenjin'” articulates well with Gal and Irvine's (1995) assertion discussed earlier, that a linguistic ideology is a totalizing vision, hence it seeks to ignore or transform elements that do not fit or that cannot be seen to fit its interpretive structure. Also, Mandumo's responses in the chart above do not acknowledge that there are some speakers from the mentioned ethnic communities who do not experience the said linguistic challenges. Neither do they account for speakers from mixed ethnic backgrounds. However, what is important in the current analysis is that such generalizations are markers of conformity to the language ideology that seeks linguistic homogeneity. The various examples discussed so far demonstrate how participants of the shrubbing discourse conform to the dominant official language ideology and the local cultural frameworks. Next, I focus on the various social

${ }^{82}$ Kales is a short form of the Kalenjin and the term is used mainly by young Kenyans. 
practices through which participants of the shrubbing discourse express resistance to the same.

\subsubsection{Resistance in the shrubbing discourse}

Both teachers and students in Nakuru and Mombasa reported that young Kenyans engaged in the shrubbing discourse in order to "bring down" speakers to the level of the audience. According to Mrs. Wanjala, the teacher from Nakuru, many young Kenyans perceived speaking Standard Swahili or English as a way of showing off or elevating oneself among peers (Mrs. Wanjala 11/2011, interview). Similarly and more specifically, Mr. Fujo from Mombasa reported that young Kenyans regarded peers who spoke English most of the time as "people who pretend to know much...people who want to prove that they know a lot of English and that they are the ones who love education most" (Mr. Fujo 01/2012, interview). For these reasons, peers expected such students to speak "pure" English and standard languages. Otherwise if they shrubbed, Mrs. Wanjala reported that some peers scorned them using utterances such as, "Ooh, so they are human beings just like us!” (Mrs. Wanjala 11/2011, interview).

Interestingly, despite young Kenyans' obsession with linguistic “correctness," they do not reward or praise those who speak Standard Swahili and English “correctly." Instead, they sometimes ridicule and label them as washamba 'country' and wasomi 'Swahili for scholars' respectively. Other times they refer to them using sarcastic tags such as "the one who speaks English" (Mr. Mgema 11/ 2011, interview). These reactions demonstrate how the shrubbing discourse brings down students and further points to its paradoxical nature. Since the examples cited so far 
demonstrate how the shrubbing discourse "brings down" students, I now focus on whether it also brings down teachers and in what ways.

According to Mr. Fujo and Mrs. Wanjala, students perceived teachers as authorities both in the officially sanctioned languages and subject content. Therefore, students did not expect them to make certain mistakes such as shrubbing. So when a teacher shrubbed, Mr. Fujo said that students questioned whether s/he was the right person to teach them. He compared this reaction to how students reacted when they realized that a teacher came to class unprepared or gave a wrong explanation to a concept. According to Mr. Fujo, "that is when students start saying, but Mwalimu ['teacher'] it was supposed to be like this! That way, they want to show everybody that the teacher is wrong" (Mr. Fujo 01/2012, interview). Such reactions indicate that shrubbing puts the affected teacher's identity and associated authority at stake. For example, I got the extract below from a handwritten weekly column called SHAMRA SHAMRA ZA SHULE YETU mentioned earlier. Recall that the author was a known innovator of Sheng and was also known for entertaining peers through ridicule and mockery. In the extract below, he depicts a certain teacher as linguistically deficient, as marked by the high degree of redundancy and the characteristic influence of her ethnic language in pronunciation. The teacher also improperly added $/ \mathrm{n} /$ in phonetic environments. The author reports:

En Finally, a prayer dedicated by Shosh $2 U s^{83}$

Oh Ndea Ngod my father njehova ngod my father. Njesus Christ my lond njehover ngodi. King of ngrore my king my father Lond njehover. Herop Mesta Prinsipal my father njehova 2 stop saying my father that we've started the day my father on a very

\footnotetext{
${ }^{83}$ Even though this sentence appears like English, speakers in Nakuru regard it as Sheng because it contains the word Shosh, which has been appropriated from Cucu (Kikuyu for grandmother) but has been linguistically manipulated to sound urbanite. One of the informants told me that they got it from a media program called "Mother-in-Law" aired on Citizen TV (Mpenda 2011, casual conversation). Also note the use of numbers, e.g., 2, in place of words, which is one of the recent linguistic innovations written in Sheng.
} 
high note my father njehover ngod King of ngrore my king we thank U njesus Christ. My father njehova ngondi (SHAMRA SHAMRA ZA SHULE YETU 11/2011, Weekly Column).

And finally, a prayer dedicated by grandmother to us

Oh dear God my father Jehovah God my father. Jesus Christ my lord Jehovah God.

King of glory my King my father lord Jehovah. Help Mr. Principal my father Jehovah to stop saying my father that we have started the day my father on a very high note my father Jehovah God my King we thank you Jesus Christ. My father Jehovah God (SHAMRA SHAMRA ZA SHULE YETU 11/2011, Weekly Column.

Participants of the shrubbing discourse also "bring down" shrubbers by nicknaming them, as reported earlier by students Hora, Bakari and Mandumo from Nakuru. Similarly, in a focus group in Mombasa, student Zawadi reported how her classmates nicknamed their Physics teacher for mispronouncing the word magnetism as [magnutusm] (Form Two focus group 2, 03/2012--Mombasa). This teacher was Luo and spoke English with mother tongue influence. In such cases, nicknaming redefined the teacher in ways that are not captured in his/her actual name and also allowed students to talk about him/her "safely." In the case of Magnutusum, I discovered that the nickname enabled students in Zawadi's class to make fun of their Physics teacher, and also to stereotype him as Luo. For example, if he punished them unfairly, they would essentialize him by saying things like "this is a true Magnutusm."

From another point of view, some informants reported that the shrubbing discourse indicates the social distance between teachers and their students. However, the discourse sometimes "brings down" the teacher to the level of the students. For example, Mr. Fujo reported that students could not laugh openly at their teachers a few decades ago because they knew they would be punished severely. However, he claimed that the social distance had narrowed over time. Thus, it was common to find students laughing and making fun of teachers who shrubbed. 
At another level, shrubbing discourse reflects how different teachers relate with students. For example, Mr. Fujo said that students laughed when he shrubbed but would not when the school Principal did it. But why was that the case? Mr. Fujo said he thought that students laughed at him when he shrubbed because they perceived him as a young man that they could make fun of. Also, he thougt that students laughed in order to torment him psychologically since there were times he punished them harshly. Similar sentiments were expressed by Mrs. Wanjala who claimed that shrubbing discourse was a way of bullying or harassing teachers, especially "those who feel sweet about themselves, those who use highly Standard Swahili or English, and those who always correct students' grammar even outside the classroom" (Mrs. Wanjala 10/2011, interview). In this regard, I argue that shrubbing discourse is a counterstrategy that students use to challenge teachers' authority. But how do teachers respond when students point out that they have shrubbed? And how do their reactions articulate with the social functions of the shrubbing discourse?

Mr. Fujo reported that he did not punish his students for laughing at him because he discovered that the more he got angry, the more they laughed at him. Instead, he said that he devised a "survival" strategy where he would allow them to laugh for a while, and then sarcastically ask them whether they were done laughing. That way, he claimed, he was able to reassert himself, hence remain in control of the class and also diffuse tension between him and the students. The way Mr. Fujo engaged his students alludes to two social functions of the shrubbing discourse. Mr. Fujo's first reaction where he stops speaking and allows students to laugh at him demonstrates that the discourse challenges his authority and 'brings him down' to the level of the students. The second account where he strategically engages the students' 
laughter indicates that he productively employs the shrubbing discourse to create a lively learning environment. The latter function was also reported by Mrs. Wanjala, who claimed that shrubbing discourse helped teachers and students to break tension in their interactions because it brought a sense of humor. For example, she reported that whenever students laughed at her for shrubbing, she in turn asked them to pronounce certain tongue twisters, especially those that she knew would trouble them. She said that the strategy not only gave her a chance to laugh at them, but also for students to laugh at themselves, further adding humor to the lesson. In this sense, teachers such as Mrs. Wanjala and Mr. Fujo appropriate shrubbing discourse as a pedagogical resource in their respective teaching subjects. In the following section, I examine the earlier claim that the current generation's notion of shrubbing is more elaborated than the older generations' notion of mother tongue interference.

\subsubsection{Ingeniousness in the shrubbing discourse}

In both Nakuru and Mombasa, speakers kept a record of peers who shrubbed and also administered a social reward system which involved both "shaming" and "faming" them. However, due to the differences in the sociolinguistic composition of the two towns, I will analyze them separately, starting with Nakuru.

\subsubsection{The shrubbing discourse in Nakuru}

In Nakuru, Shrubbing discourse was more conventionalized than Mombasa and also more elaborated than the older generations' notion of mother tongue interference. Peers administered a negative social reward system where in addition to ridiculing the shrubber, the person who witnessed the shrubbing documented his/her observations on a section of the classroom wall that peers called the "Wall of Shame." 
The details entered included: name of the shrubber, what s/he said, and what s/he ought to have said. All these details were entered in columns that facilitated tracking of frequent shrubbers (both teachers and students) over a given period of time. However, if these individuals became frequent shrubbers, their names were transferred to another section of the classroom wall that peers called "Wall of Fame." Aside from its use in the shrubbing context, the "Wall of Fame" was a social space where names and details of individuals who had remarkable achievements - such as excelling at sports - were documented. In a Facebook follow-up conversation with one of my student informants, Kwatokata, he described the two walls as follows ${ }^{84}$ :

MW: I am luking ${ }^{85}$ 'looking' for comments about the "Wall of Shame" and Wall of Fame that I saw in your classroom.

Kwatokata: Well, in class we had two opposite walls for the two purposes.

1. WALL OF SHAME

Here was the place where the poor pronouncers were. Students with mother tongue influences were put here. When someone mistakenly mispronounces a word, it's a source of humour to the class. Teachers who shrubb were no exception from the list either although it was kept a secret. The benefits of the wall is that when speaking, one takes precaution to avoid errors which lead to embarrassments. In so doing, the students even improve in language lessons as it turned to be seen later in our class, that is.

\section{WALL OF FAME}

Here is where the individual achievements of the class were posted. They included the worst noisemaker where I always led lol [English Slang for 'laughing out loud'], the best scorer in football and rugby etc. The benefits of this particular wall enhanced hard work in co-curricular activities amongst ourselves.

MW: One of the students had told me that those who shrubbed all the time/continuously were transferred from "Wall of Shame" to "Wall of Fame." Is that true?

Kwatokata: Yah when u shrub too much u bcome like a superstar hadi ukishrub watu wamekuzoea and dont consider you anymore ['Yes, when you shrub too much, u become like a superstar such that people get used to you such that even if you shrub, they no longer bother about you'] (Kwatokata 07/2013, First Facebook interview).

\footnotetext{
${ }^{84}$ Note that I have maintained Kwatokata's spelling.

${ }^{85}$ This spelling was motivated by my desire to express solidarity with Kwatokata.
} 
Tables 2 and 3 below are extracts from the "Wall of Shame" and "Wall of Fame" in the Nakuru School.

Table 2: The "Wall of Shame" (spellings of shrubbed words and corrections as found on the Wall; names are pseudonyms).

\begin{tabular}{|c|c|c|c|}
\hline $\begin{array}{l}\text { NAME (of } \\
\text { shrubber) }\end{array}$ & Shrubbed Word(s) & "Correct"/ Expected & $\begin{array}{l}\text { Notable Sounds/Features } \\
\text { involved }\end{array}$ \\
\hline Dot & Blush & Brush & $/ 1 /$ and $/ \mathrm{r} /$ \\
\hline Bramblee & Shoo & Choo & $/ \mathrm{g} /$ and $/ \mathrm{t} \mathrm{f} /$ \\
\hline Garang & Kuchoto & Kushoto & $/ \mathrm{g} /$ and $/ \mathrm{t} \mathrm{f} /$ \\
\hline Mose & Loli & Lorry & $/ 1 /$ and $/ \mathrm{r} /$ \\
\hline Kibe & Kalatasi & Karatasi & $/ 1 /$ and $/ \mathrm{r} /$ \\
\hline $\begin{array}{l}\text { [Principal's } \\
\text { nickname] }\end{array}$ & Paur & Paul & $/ \mathrm{l} /$ and $/ \mathrm{r} /$ \\
\hline $\begin{array}{l}\text { [Principal's } \\
\text { nickname] }\end{array}$ & Owuol & Owuor & $/ \mathrm{l} /$ and $/ \mathrm{r} /$ \\
\hline Kihumba & Shaili & Shairi & $/ / 1 /$ and $/ \mathrm{r} /$ \\
\hline Davy (Dosi) & Brankets & Blankets & $/ \mathrm{l} /$ and $/ \mathrm{r} /$ \\
\hline Kibe & Kasi & Kazi & $/ \mathrm{s} /$ and $/ \mathrm{z} /$ \\
\hline Joyce (Wa West) & Funja & Vunja & $/ \mathrm{f} /$ and $/ \mathrm{v} /$ \\
\hline Emily & Londa & Ronda & $/ \mathrm{l} /$ and $/ \mathrm{r} /$ \\
\hline Joyce & Nishaidie & Nisaidie & $/ / /$ and $/ \mathrm{s} /$ \\
\hline Oralo & Tarasa & Darasa & $/ \mathrm{t} /$ and $/ \mathrm{d} /$ \\
\hline Emily & Ngorofa & Gorofa & $/ \mathrm{gg} /$ and $/ \mathrm{g} /$ \\
\hline Rose & Nakuonesanga & Nakuoneshanga & $/ \mathrm{s} /$ and $/ \mathrm{g} /$ \\
\hline Rose & Afsan & Afshan & $/ \mathrm{s} /$ and $/ \mathrm{g} /$ \\
\hline Ann & Lare & Rare & $/ \mathrm{l} /$ and $/ \mathrm{r} /$ \\
\hline Rose & Mishing & Missing & $/ \mathrm{g} /$ and $/ \mathrm{s} /$ \\
\hline Ann & Meri & Meli & $/ 1 /$ and $/ \mathrm{r} /$ \\
\hline Maggie (Prefect) & Shaili & Shairi & $/ \mathrm{l} /$ and $/ \mathrm{r} /$ \\
\hline Mercy & Ukerekeretaji & Ukeretaji & Root doubling \\
\hline Joyce & Kushukua & Kuchukua & $/ \mathrm{f} /$ and $/ \mathrm{t} \mathrm{f} /$ \\
\hline Ann & Tombik & Topic & $/ \mathrm{mb} /$ and $/ \mathrm{p} /$ \\
\hline Ann & Sine & Shine & $/ \mathrm{s} /$ and $/ \mathrm{g} /$ \\
\hline Ann & Arone & Alone & $/ 1 /$ and $/ \mathrm{r} /$ \\
\hline Mr. Luhalango & Jesus crust & Jesus Christ & /u/ and /i/ \\
\hline Alex Maangi & She alsho & She also & $/ \mathrm{S} /$ and $/ \mathrm{s} /$ \\
\hline Hoof cutter & Langi & Rangi & $/ 1 /$ and $/ \mathrm{r} /$ \\
\hline *Cancelled/Erased* & Rara & Lala & $/ 1 /$ and $/ \mathrm{r} /$ \\
\hline Barasa & Erectrostatic & Electrostatic & $/ \mathrm{l} /$ and $/ \mathrm{r} /$ \\
\hline Ouma & Arrow & Allow & $/ \mathrm{l} /$ and $/ \mathrm{r} /$ \\
\hline Hoof cutter & Crue & Clue & $/ 1 /$ and $/ \mathrm{r} /$ \\
\hline Mary & Wachaluo & \begin{tabular}{|l|} 
Wajaluo \\
\end{tabular} & $/ \mathrm{t} / /$ and $/ \mathrm{d}_{3} /$ \\
\hline Alex & Sarp & Sharp & $/ \mathrm{s} /$ and $/ \mathrm{g} /$ \\
\hline Nduta & Njirani & \begin{tabular}{|l|} 
Jirani \\
\end{tabular} & $/ \mathrm{nd} \overline{d_{3} / \text { and } / \mathrm{d}_{3} /}$ \\
\hline $\begin{array}{l}\text { SNA Robert A.K.A } \\
\text { Mr. Prefect }\end{array}$ & Englis & English & $/ \mathrm{s} /$ and $/ \mathrm{g} /$ \\
\hline Nduta & Biro & Bilo & $/ 1 /$ and $/ \mathrm{r} /$ \\
\hline Kirika & Lesscross & Redcross & $/ \mathrm{s} /$ and $/ \mathrm{d} /$ \\
\hline Siva & Nasangaa & Nashangaa & $/ \mathrm{s} /$ and $/ \mathrm{J} /$ \\
\hline SNA & Umechinda & Umeshinda & $/ \mathrm{t} f /$ and $/ \mathrm{s} /$ \\
\hline Alice & Nitoree & Nitolee & $/ \mathrm{l} /$ and $/ \mathrm{r} /$ \\
\hline Joyce & Kuchola & Kuchora & $/ \mathrm{l} /$ and $/ \mathrm{r} /$ \\
\hline
\end{tabular}




\begin{tabular}{|l|l|l|l|}
\hline Lewo & Poys & Boys & $/ \mathrm{p} /$ and /b/ \\
\hline Alfa & Nchinini & Nchini & Doubling the "-ni" locative \\
\hline Lewo & Seng & Sheng & $/ \mathrm{s} /$ and $/ \mathrm{g} /$ \\
\hline *Unknown* & Maza & Matha & $/ \mathrm{z} /$ and $/ \mathrm{d} /$ \\
\hline Lilian & Fulanzi & Fulani & $/ \mathrm{n} /$ adding \\
\hline Njenga & Chukua iso & Chukua hizo & $/ \mathrm{h} /$ Dropping \\
\hline Lilian & Kishahili & Kishairi & $/ \mathrm{l} /$ and $/ \mathrm{r} /$ \\
\hline Ghandi & Decate & Decade & $/ \mathrm{t} /$ and $/ \mathrm{d} /$ \\
\hline
\end{tabular}

Speakers, Ann, Joyce and Rose in the "Wall of Shame" above, were identified as frequent shrubbers, hence their names were transferred to the wall of "Fame" below:

Table 3: The "Wall of Fame" (spellings as found on Wall; names are pseudonyms).

\begin{tabular}{|l|l|l|l|}
\hline NAME & Shrubbed word & $\begin{array}{l}\text { "Correct"/ } \\
\text { Expected }\end{array}$ & $\begin{array}{l}\text { Notable Sounds/Features } \\
\text { involved }\end{array}$ \\
\hline \multirow{4}{*}{ Ann } & Lare & Rare & $/ \mathrm{l} /$ and $/ \mathrm{r} /$ \\
\cline { 2 - 4 } & Meri & Meli & $/ \mathrm{l} /$ and $/ \mathrm{r} /$ \\
\cline { 2 - 4 } & Tombik & Topic & $/ \mathrm{mb} /$ and $/ \mathrm{p} /$ \\
\cline { 2 - 4 } & Sine & Shine & $/ \mathrm{s} /$ and $/ \mathrm{s} /$ \\
\cline { 2 - 4 } Joyce & Arone & Alone & $/ \mathrm{l} /$ and $/ \mathrm{r} /$ \\
\hline \multirow{3}{*}{ Rose } & Nishaidie & Nisaidie & $/ \mathrm{s} /$ and $/ \mathrm{s} /$ \\
\cline { 2 - 4 } & Kushukua & Kuchukua & $/ \mathrm{s} /$ and $/ \mathrm{t} /$ \\
\cline { 2 - 4 } & Kuchola & Kuchora & $/ \mathrm{l} /$ and $/ \mathrm{r} /$ \\
\hline & Nakuonesanga & Nakuoneshanga & $/ \mathrm{s} /$ and $/ \mathrm{s} /$ \\
\cline { 2 - 4 } & Afsan & Afshan & $/ \mathrm{s} /$ and $/ \mathrm{s} /$ \\
\cline { 2 - 4 } & Mishing & Missing & $/ \mathrm{s} /$ and $/ \mathrm{s} /$ \\
\hline
\end{tabular}

A closer look at Kwatokata's response in the excerpt further above reveals that the transition from the "Wall of Shame" to "Wall of Fame" is paradoxical. From one point of view, students who participated in the shrubbing discourse reported that shrubbing was a marker of linguistic deficiency; hence first time shrubbers and associated details were entered into the "Wall of Shame." These students also reported that frequent shrubbing earned individuals "fame" among peers, hence the transfer to "The Wall of Fame." However, what these students called "fame" in the context of shrubbing was perceived by others as mockery. With regard to "fame," 
some participants perceived the transfer from the "Wall of Shame" to the "Wall of Fame" as an achievement. This perception was informed by the fact that the "Wall of Fame" was a social space where high achievers in different fields were listed. For example, student Kadogo from Nakuru reported that:

In our school for example, there, shrubbing is such a big deal that students write what you mispronounce on the wall known as "Wall of Shame" while those who use vocabulary [difficult words or those unknown to others] are recorded on the other side of the classroom known as "Wall of Fame." Students here are so keen when giving speeches so that they do not find themselves appear on the "Wall of Shame" but when they appear on the "wall of fame" someone is proud of themselves. (Kadogo 07/2013, Email)

But how would one become a "superstar" from excelling in a social practice that is highly stigmatized among peers? Also, what does Kwatokata mean by his claim that peers get used to frequent shrubbers, hence they no longer bother about them? A critical look at the shrubbing discourse reveals that it is organized around the notion of "community of practice" (Eckert and McConnell-Ginet 1992), discussed in Chapter Two. In this regard, some of the meanings attached to the shrubbing discourse including the notions of "shame" and "fame" - are only decipherable to the in-group members. In another follow-up interview with Kwatokata over Facebook, he elaborated what it meant to become a "superstar" and clarified that the shrubbing discourse was an in-group social practice. In his view (note that I have maintained Katokata's spelling):

The main aim besides correcting someone is the fun part. So, when you shrub exceedingly, the attention shifts from you due to monotony. It was much more fun if someone who didn't shrub daily to be caught. U ['you'] become a superstar in the sense that it's every time when it comes to you. So, people get used to you, but someone who does not shrub it's always exciting to hear new stuff completely different from the daily one. Becoming the "superster" ['superstar'] in this perspective is different from that of a celeb ['celebrity']. Mostly it's a classroom affair and not beyond. (Kwatokata 07/2013, second Facebook interview)

In spite of the lesser attention accorded to speakers in the "Wall of Fame," it is interesting to note that Kwatokata later explained that their fame kept on 
accumulating. This is because whenever new speakers were added to the "Wall of Shame," some were transferred to the "Wall of Fame." And since names of the new transferees were added at the bottom of the "Wall of Fame," names kept on going up the ladder, suggesting that the higher a speaker's name was on the list, the more fame s/he had accumulated.

In some contexts, my research findings indicate that transfer from "The Wall of Shame" to "The Wall of Fame" sometimes symbolized condemnation of the affected individuals in that they could not speak "pure" Standard Swahili or English as they purported to do. Due to the fear of being ridiculed, hence loss of face (Goffman 1959), speakers suffered from "linguistic insecurity," which is a state of anxiety and nervousness about the "correct" use of language (Bourdieu 1977; Labov 1966, 1972). Such anxiety often led speakers to make mistakes that they would not otherwise make; further prompting peers to laugh at them. In such cases, the shrubbing discourse discouraged speakers from employing Standard Swahili and English during peer interactions. For example, the student Kadogo from Nakuru reported that shrubbing, "huwa inalower self esteem ya mtu na kumfanya mtu mwoga wa kuongea mbele ya watu”, 'Shrubbing lowers speaker's self esteem and makes him/her develop the fear of speaking in public' (Kadogo 07/2013, Email).

Since Sheng draws some of its linguistic material from ethnic languages, does shrubbing occur in Sheng? One category of speakers claims that shrubbing does not occur in Sheng because Sheng deletes the effect of mother tongue influence in one's speech as in the case of /r/ and /1/ for Kikuyus. For example, recall student Munne from Nakuru, in the quotation at the beginning of this chapter, who reported that Sheng provided him alternative lexical items, which helped him escape embarrassing 
situations since he had problems distinguishing between /r/ and /1/. Specifically, he said that in case he wanted to say kulala 'to sleep', he would instead say kutuna (Form Four focus group 4, 11/2011--Nakuru). Another category of students from Nakuru claimed that since Sheng is a mixture of various languages, it was hard for listeners to detect mother tongue influence when one spoke. As such, speakers claimed that shrubbing in Sheng could be hidden, and could also be part of Sheng. This makes it hard to know when someone has shrubbed in Sheng (Praise and Worship focus group, 11/2011--Nakuru).

However, there were students who claimed that shrubbing did occur in Sheng. The only difference was that peers' responses were usually biased depending on who had shrubbed. For example, student Mailinne from Nakuru reported that most of the Sheng innovators were "cool" guys in the "streets," or individuals who were popular among the youth. ${ }^{86}$ Therefore, even if they shrubbed, theirs would come out as "style" or "design" due to their influential positions in the Sheng speaking network. As such, some people would adopt their shrubbing as new Sheng and start using it. However, if an ordinary person shrubbed, people would laugh at him/her (Form Four focus group 1, 11/2011--Nakuru). What Mailinne called "style" or "design" is the notion of swag introduced earlier. While swag refers to an individual's or group's unique way of engaging in a social practice, there are students who use it as a salvation device or a mask to save face socially when caught shrubbing. For example, if an individual were told by peers that he had shrubbed, s/he would tell them that that was his/her swag as

\footnotetext{
${ }^{86}$ Street in this context should be understood as a site of linguistic innovation rather than physical space that the wider Kenyan society associates with dodgy fellows and gangster activities.
} 
a way of socially saving face. This individual would become more cautious in future conversations in order to avoid being caught shrubbing again.

The discussion so far has focused on the notion of shrubbing among young Kenyans in Nakuru. In the following section, I focus on the shrubbing discourse among speakers of lugha ya mtaa in Mombasa, seeking to establish whether there are notable differences from that of Sheng speakers in Nakuru.

\subsubsection{The notion of shrubbing among speakers of lugha ya mtaa in Mombasa}

The sociolinguistic situation of Mombasa is complex. Despite the fact that many of the communities that make up the Mombasa population have lived there for a long time, speakers make numerous insider-outsider distinctions in their interactions surrounding the question "Who is Mswahili?" I will limit my focus to the broadest categorization in which members of the ethnic communities who regard themselves as Waswahili pejoratively refer to other communities as watokabara 'from upcountry'. In the earlier discussion, I noted that some young Kenyans in Mombasa claimed that they spoke Sheng, others lugha ya mtaa, while others claimed that they spoke both. Speakers in all categories expressed similar sentiments as their counterparts in Nakuru about the causes of shrubbing and associated meanings. However, speakers of lugha ya mtaa in Mombasa, gave an additional social meaning, saying that shrubbing marked the speaker as mtokabara. This is well captured in the following extract from a Facebook follow-up interview that I had with a student, Mose, whose family is from upcountry but resides in Mombasa: 
1. MW: Na watu wa coast huwa na hii stori ya shrubbing? Yaani utapata mavijanaa wakipoint out eti umeshrub or such?

2. Mose: Watu wa Coast speak fluent Swahili. So they call as wabara cos of the way we speak; our Swahili is different.
1. MW: Do people from Coast talk about shrubbing? That is, would you find peers pointing out that one of them has shrubbed or such?

2. Mose: People from Coast speak fluent Swahili. So, they call us "people from upcountry," because of the way we speak; our Swahili is different.

(Mose 03/2013, Facebook interview)

The other notable feature of the shrubbing discourse in Mombasa is that speakers do not regard the influence of Kimvita as shrubbing. Instead, my research data suggests that it only applies when one speaks Swahili or English with the influence of upcountry ethnic languages. ${ }^{87}$ This is contradictory because Kimvita is a mother tongue to some students, and it is one of the "errors" that teachers correct in students' spoken and written language. Moreover, even though some speakers of lugha ya mtaa praised Zanzibar Swahili, that is, Standard Swahili, as in the case of Zawadi discussed in Chapter Four, participants of the shrubbing discourse did not reward those who spoke it "correctly." This paradox is rooted in the fact that Kimvita accent accords a speaker the highly regarded coastal local identity within the Mombasa Swahili speaking community. Again, I noted in Chapter Four that many young Kenyans from both Nakuru and Mombasa reported that they found coastal Swahili "sweet," hence they enjoyed listening to it.

The practice of documenting shrubbers in Mombasa was not as elaborate as in Nakuru and participants did it privately rather than on classroom walls. For example, some speakers reported that it was only practiced among close friends who documented their observations either in their notebooks, in their memory or on walls

\footnotetext{
${ }^{87}$ This is a question that would require further research because though I did not record any instance of shrubbing from the influence of non-Swahili coastal languages, I did record an instance of Wagiriyama being regarded as washamba.
} 
of buildings in their residential neighborhoods close to where their social groups usually met. Possibly, this is because the Mombasa school prohibited students from writing on classroom walls and severely punished those who violated this rule.

However, despite the restricted nature of the shrubbing discourse in Mombasa, it still embodied the same nuances of "shame" and "fame" as in Nakuru. It was also organized around the notion of community of practice, with variations in meaning between various social groups. In addition, though speakers did not report that they employed the "Walls" of shame and "fame," the process of tracking shrubbers followed a similar progression as in Nakuru. That is, first time shrubbers were shamed instantly and their names and details of what they shrubbed documented. Should they shrub again, peers reminded them that they had shrubbed before, and sometimes informed them about who else had shrubbed like them. However, if peers realized that some speakers had become frequent shrubbers, they labeled them "Masters of Shrubbing." These attributes are demonstrated in the transcript below from one of the mixed-gender focus groups:

1. MW: Na mbona mtu "akishrub" huwa anachekelewa? Kwa nini? Why is it amusing to them?

2. Msha: Kwa sababu wataona anafanya kimakusudi, nini. Kwa sababu wengine hawatajua kama huyu ni shida yake ya mother tongue lakini wale watacheka tu. Na ataona kama yuaonelewa.

3. Wamsanifu: Watakuwa hawaelewi. Tena watamcheka. Akiwauliza "mwacheka nini?" Watamwambia, "washema kweli!"

4. Mshah: Hata siku hizi kuna watu wameanza instead of kusema "sema" wanasema "shema" words. Ndio, wanatafuta zile shema words ambao

5. MW: Ooh kuna shema words?

6. Mshah: Ah ah, sijui lakini kuna watu wameanza; kama mtu akikosea wanaandika.
1. MW: And why do peers laugh when someone shrubs? Why? Why is it amusing to them?

2. Msha: Because they think it is deliberate since they do not know whether one has a problem of mother tongue influence. As such, they would just laugh and the person being laughed at would think peers are picking on him.

3. Wamsanifu: They laugh because they do not understand. If the person being laughed at asks them why they are laughing? They would tell him, "you are really 'shrubbing!',"

4. Mshah: Even nowadays, instead of saying "sema," peers say, "shema" words. Yes, they listen to words that have been shrubbed.

5. MW: Ooh you mean there are "shema" words?

6. Mshah: Ah ah, I don't know but peers have started let's say someone has erred when speaking, they note down that. 
7. MW: Ooh, na wanaandika wapi?

8. Mshah: Kwa kitabu, ni peke yao, wanafunzi peke yao.

9. Mshefad: Hata nyumbani kwetu, "ukishrub" unaandikwa. Kuna pahali kwa nyumba ya askari, saa ukikosea hivi kidogo, wanaandika pale.

\section{MW: Kwenu nyumbani?}

11. Mshefad: Ah ah, yani pale kwa estate. Huwa kuna zile guard za askari. Kule kwenye huwa wanaketi. Sasa huko ndiko huwa tunaketi sana. Sasa huwa wanaandika.

12. MW: Na ni kwa nini? Unaandikwa kwa ukuta kwa sababu umeshrub?

13. Mshefad: Mmh

14. MW: Na utaandikwaje? How will they put it down?

15. Mshefad: Tuseme ulikuwa wataka kusema book ukasema “mbook," sasa wanakuandika John.

16. Mshah: Hata mimi saa hii na rafiki yangu tunaandika lakini mimi sasa kuna siku naenda kule instead ya niseme vulture nikasema "aulture," sasa ikaandikwa.

17. MW: Iliandikwa kwa ukuta?

18. Mshah: Ah ah kwa kitabu. Ni sisi wawili peke yake.

19. MW: Ndio mnaandikana?

20. Mshefad: Eeh

21. MW: And eh why is it interesting to take note of shrubbing? Mbona inavutia?

22. Mshefad: Us are doing it for correcting. For correction.

23. MW: For corrections?

24. Mshefad: Yah.

25. Wamsanifu: By the time mtu akikosea tena unamwambia, "mwenzako pia alisema hivi." Na wewe wamwandika tena.

26. MW: Ooh

27. Wamsanifu: Kama mtu akikosea kwa mfano, "she shells the shea shells at the shea shore," you will be told, "Washema kweli." Aah, master wa kushema!

28. MW: Eeh, unaitwa Master wa kushema?

29. Wamsanifu: Mmh
7. MW: Ooh, and where do they write?

8. Mshah: In a book but just among them.

9. Mshefad: Even in my residential neighborhood, if you shrub, peers note it down. There is a place at the police post/camp where peers document whenever someone makes a slight error when speaking.

10. MW: In your neighborhood?

11. Mshefad: Ah ah, yes in my residential estate/neighborhood. At a place where police officers like hanging out. That is where my social group hangs out and document details of peers who shrub.

12. MW: But why? Is your name written on the wall because you shrubbed?

13. Mshefad: Mmh

14. MW: And how will they put it down?

15. Mshefad: Let's say you intended to say "book" but instead said "mbook," they will write your name,e.g., John, and what you shrubbed.

16. Mshah: Even me now, my friend and I usually write. One day, I intended to say [vulture] but instead said [auture]. My friend wrote it down.

17. MW: And was it written on a wall?

18. Mshah: No! No! In a book. It is only between us two.

19. MW: You only do it between you two?

20. Mshefad: Eeh

21. MW: And why is it interesting to take note of shrubbing? Why is it interesting?

22. Mshefad: We do it in order to correct each other's language.

23. MW: For corrections?

24. Mshefad: Yah.

25. Wamsanifu: So, if somebody shrubs again, you inform him that his/her friend had also shrubbed and then add this incident to his existing records.

26. MW: Ooh

27. Wamsanifu: If somebody mispronounces the following words "she sells sea shells at the sea shore," s/he will be ridiculed, and be told, "You are really 'shrubbing.' Aah, you are a master of "shrubbing.",

28. MW: Eeh, you are labeled master of 'shrubbing?

30. Wamsanifu: Mmh.

(Form One focus group, 03/2012--Mombasa) 
In summary, the shrubbing discourse involves mocking speakers who do not use the officially approved languages without any "errors," and disapproving those who do use the approved languages for showing off. Both strategies, however, aim at discouraging peers from using the officially sanctioned Standard Swahili and English in favor of the unauthorized nonstandard language varieties such as Sheng and lugha ya mtaa. Speakers also employ shrubbing discourse to express disdain for ethnic languages. However, the discourse is paradoxical because participants simultaneously associate and dissociate with Standard Swahili, English and ethnic languages. Specifically, while peers reprimand shrubbers for not displaying the expected linguistic "correctness," they do not reward/praise those who speak Standard Swahili and English "correctly." Instead, as noted earlier, they ridicule them using labels such as washamba and wasomi. Interestingly, there are some participants who claimed that frequent shrubbing generated "fame" or made one a "superstar." In the next section, I discuss situations when this shrubbing notoriety translated into symbolic capital.

\subsubsection{Shrubbing as symbolic capital}

Earlier in the chapter, I reported the case of student Mwelusi who said that some of his friends would deliberately shrub sounds not associated with their perceived ethnic communities in order to create humor among peers. This practice indicates that shrubbing to some participants is a performance and a marker of ingeniousness. Recently, though outside my two research sites, a young Kenyan called Joseph Mburu was captured shrubbing in a media interview while he narrated how he witnessed a criminal incident in Nairobi. Part of what he said was, "I witnessed! Ni kama nndrama, ni kama vindeo!", 'I witnessed! It is like drama, it is like 
video' ${ }^{88}$ Even though his shrubbing in this particular case was not deliberate, many young Kenyans found it funny and entertaining. Following his media interview, Joseph became well known (what participants of the shrubbing discourse call famous) among many young Kenyans, especially in the social media. Some of these young Kenyans renamed him "Jose the Witnesser!" They also created several Facebook pages and Twitter accounts under the name "I witnessed! Ni kama nVindeo!" Ultimately, "Jose the Witnesser" became a household name among many young Kenyans. Some adopted his expression "Ni kama ndrama, ni kama Vindeo!” as a slogan for making fun of incidents that they found ridiculous or hard to comprehend. For instance, if a team lost a soccer match by a big margin, some soccer fans may ridicule fans of the losing team by saying the match "Ni kama ndrama,ni kama vindeo"; implying that things were moving too fast for the losers. Politicians and renowned televangelists began inviting Jose to entertain youths at political rallies and to open air church crusades, further adding to his fame as a shrubber. Ultimately "Jose The Witnesser" became very popular among young Kenyans for his conspicuous shrubbing and seemed to enjoy and benefit from the increased media attention. He even confessed in a media interview that this media attention had led to an increase in sales in his milk-selling business in Nairobi, because people had been frequenting his store seeking to be entertained and many of them bought milk on their way out.

Other than "Jose the Witnesser," there is a popular entertainer, "Teacher Wanjiku," who employs shrubbing for humor. She is a leading performing artist in one of Kenya's leading standup comedies called The Churchill Show aired on

\footnotetext{
${ }^{88}$ Note the addition of $/ \mathrm{n} /$ in the words 'video' and 'drama'. Watch Joseph's popularity video here: http://www.youtube.com/watch?featureplayer_embeddedandvQQYtGZ_gDR4
} 
Nationwide Television Network (NTV). “Teacher Wanjiku” entertains through ethnic mockery, ridiculing teachers of Swahili in Kenyan schools, and projecting them as people with serious mother tongue influence which interferes with their teaching. ${ }^{89}$ She artfully draws on ethnically marked pronunciations; especially those associated with Kikuyu, her own ethnic background, and uses them to parody teachers of Swahili in Kenya in ways that draw in her non-Kikuyu listeners in the humor attached to these stereotypes. In the video shared in footnote 89 , teacher Wanjiku emphasizes the trilled [r] sound that I alluded to earlier, and also puts it in places where [1] would be "correct." She also pronounces $\left[\int\right]$ in places of $[\mathrm{t} f]$ and vice versa. The various examples demonstrate that shrubbing is a social asset to some speakers. In addition, the discourse suggests that it is a way of appreciating ethnic diversity. This challenges the national language ideology in Kenya, which socially constructs ethno-linguistic diversity as a threat to national unity.

\subsubsection{Conclusion}

This chapter has examined how the shrubbing discourse common among young Kenyans relates to the dominant "standard language ideology" on the one hand and how it articulates with speakers' desired social identities on the other. The discussion has established that what young Kenyans call shrubbing is recognized among the older generations as mother tongue interference, but the current generation is more invested in highlighting the practice. The discussion also demonstrated that shrubbing discourse is a performance in which speakers mock and stereotype mother tongue interference in ways that suggest both resistance and conformity to the

\footnotetext{
${ }^{89}$ Here is one of the video clips about teacher Wanjiku: http://www.youtube.com/watch?v=ajxJoHGl$7 \mathrm{k}$
} 
dominant "standard language ideology" and local cultural frameworks. As a result, the discourse is replete with many paradoxes. For example, speakers engage in the discourse to mark distance between their desired urban, post-ethnic identity and the existing local cultural frameworks. Specifically, these speakers seek to differentiate themselves from their rural counterparts and the older generations by socially constructing ethnic languages and rural life as bad, and markers of backwardness. Instead, they embrace nonstandard language varieties such as Sheng and associated identities as alternatives. This is well captured in the opening quotation where student Munne's sentiments suggest that peers prefer speakers who mix languages and regard them as "trendy." Paradoxically, Sheng and lugha ya mtaa draw their linguistic materials from the same languages that speakers are seeking to dissociate from.

The discussion also established that speakers engage in the shrubbing discourse in order to "bring down" the adherents of the dominant "standard language ideology" in favor of nonstandard language varieties such as Sheng and lugha ya mtaa. However, even though participants criticize peers who speak Standard Swahili and English with mother tongue interference, speakers of lugha ya mtaa in Mombasa do not regard the influence of Kimvita as shrubbing. This paradox is rooted in the fact that local coastal identity is highly regarded among speakers of Swahili in Mombasa and it may be the reason why those who perceive themselves as Waswahili, pejoratively refer to speakers of ethnic languages associated with upcountry as watokabara. This label, as mentioned in Chapter Four, has multiple exclusionary social meanings that coastal communities use to stereotype the people they refer to.

Even though peers require speakers to employ English and Standard Swahili "correctly," they do not reward those who do so. Instead, they ridicule and mock these 
speakers, claiming that they are showing off. Similarly, despite criticizing speakers who use ethnic languages or those with mother tongue influence, the preferred Sheng draws heavily from these same languages, which raises the question of the potential for shrubbing in Sheng. Some speakers who admitted that shrubbing did occur in Sheng claimed that it could be reinterpreted as individual speaker's swag, which cushions the shrubber from being mocked. These contradictions point to the fact that speakers of youth vernaculars such as Sheng and lugha ya mtaa operate within a "paradox of norms" (Kiesling and Mous 2004), where they have to associate and dissociate with their host societies. In this regard, speakers are usually torn between pleasing peers for the purposes of solidarity, and pleasing figures of authority such as parents and teachers. As a strategy for negotiating the required balance, the discussion established that the shrubbing discourse is organized around the notion of community of practice, which allows speakers to belong to multiple social groups with different and sometimes opposing values and interests. As speakers move in out of the various communities of practice, the discussion established that they both challenge and reinforce the dominant official language ideology and local cultural frameworks. 


\section{Chapter Eight}

\subsection{Conclusion}

In this chapter, I summarize the main arguments made throughout the dissertation. Also, I propose further areas of research about Sheng and lugha ya mtaa, which were relevant to my research project but could not be addressed due to limitations of time, scope and finances.

\subsubsection{Summary of the main arguments and conclusions}

From the research study I conducted in Nakuru and Mombasa, I came up with the following significant findings which contribute to the study of the language varieties used by urban youth in Kenya. These can be used to inform the language planning and formulation of educational policies in Kenya, and other multilingual countries within and outside Africa. With regard to Sheng, my research study found that different categories of speakers had different understandings of what Sheng is, and each of these categories foregrounded different social and linguistic factors in their definitions. Even those with similar definitions sometimes differed on what would count as Sheng. Also, some speakers would associate with Sheng in some contexts but deny that they spoke it in others. I concluded that Sheng is socially defined. Thus, previous attempts to fit it into the existing linguistic criteria were inadequate because they could not account for social factors such as speakers' language attitudes, language ideology and desired social identities. These factors play a significant role in how speakers perceive, employ and define Sheng.

I argued that Irvine's (2001: 23) notion of style as a "social semiosis of distinctiveness" is the most appropriate analytical tool, since it takes into account both 
social and linguistic factors that speakers employ in their definitions, and also accommodates the wide range of speakers' understandings of what Sheng is.

Closely-related to the finding above is that not all urban youth speak Sheng on the one hand, and on the other hand, not all language varieties spoken by urban youth are called Sheng. Instead, different categories of urban youth employ different labels to refer to the language variety that they speak such as Sheng, Kikostoo, KiSheng and lugha ya mtaa. For these reasons, my friends who had steered me to Nairobi claiming that I would not find Sheng in Nakuru and Mombasa, were correct to some extent. In Nakuru, informants in the early days of my research described what they spoke as "Swahili," but on further acquaintance described it as Sheng. In Mombasa, speakers used a wide variety of terms to describe their everyday language, and they tended to associate Sheng with Nairobi. However, it was still worth doing the study in both places because even not finding Sheng was an interesting discovery, as it prompted me to interrogate the underlying factors preventing the diffusion of Sheng. Even though speakers employed different labels to define the variety they spoke, I found that Sheng was the most commonly used label in Nakuru and lugha ya mtaa in Mombasa. I attributed this distinction to speakers' desire to negotiate local identity. For example, in Nakuru, though some teachers claimed that the youth who speak Sheng there were copying Nairobi youth, some speakers of Sheng claimed that their Sheng is different from that spoken in Nairobi. They even gave examples of Sheng that they had coined themselves such as kadete 'act of talking too much' and tintole 'darkness'.

In Mombasa, the local identity was negotiated mainly along the coastalupcountry distinction, where those who perceived themselves as coastal associated 
with lugha ya mtaa while those from the communities perceived as from upcountry associated with Sheng. So, speakers' association with any of these language varieties promotes in-group solidarity, and also creates a variety of "us" vs. "them" distinctions such as rural-urban, young-old, coastal-upcountry, and tough-soft. These oppositional identities are renegotiated and maintained through "acts of alterity" (Newell 2009), where speakers of one language variety negate the attributes of others. Use of these acts of alterity was well demonstrated by Sheng speakers' reference to the style of coastal youth as KiSheng to mark it as lesser Sheng. Similarly, speakers of lugha ya mtaa in Mombasa claimed that Mombasa youth were "tough" and "independent," while describing Nairobi youth as "Mama's boys," meaning "soft" and "not independent."

Despite speakers' desire for distinctiveness, I found that some informants associated with more than one language variety, and their attitudes toward the various languages spoken in their environment were contextual. I attributed these contextual attitudes to membership in multiple communities of practice which had competing values and interests. For example, students who held leadership positions had to associate with both the officially sanctioned English and Standard Swahili on the one hand, and with the unauthorized language varieties on the other. They did thusin order to maintain a balance between pleasing school authorities and peer solidarity.

From the point of view of the national language policy, my study found that there is a disconnect between the policy in theory and practice. The discussion on the history of the formal education and associated language policy in Kenya demonstrated that the choice of a language for national functions in most situations is influenced more by the interests of the ruling class, rather than factors that apply to intended 
speakers such as their communicative needs and desired social identities. This was well demonstrated by how colonial authorities withheld the teaching of English in "African" schools despite the economic advantages it would bestow. Similarly, the post-independence government recently designated Standard Swahili as an official language despite the fact that it bestows very little economic advantage.

The discussion also demonstrated that the state only responds to issues of language when it feels threatened and abandons the project as soon as the political “dust” settles (Mutahi 1979). This practice suggests that the state does not rank language policy at the top among other priorities. This is partly the reason why there have been many policy recommendations in Kenya since the colonial period, but only those serving the interests of the government have been implemented. Such an approach to language issues has especially affected the utility of Standard Swahili both as an official and a national language. Specifically, people regard English as more official than Standard Swahili on the one hand. On the other hand, it is nonstandard varieties of Swahili among the adults, and stylistic forms such as Sheng and lugha ya mtaa among many young Kenyans, that serve the intended role of a national language in actual practice.

While the government chose Standard Swahili as a national language partly because of its perceived ethnic neutrality, I demonstrated that the language is not neutral in actuality. For example, speakers of the Kimvita variety in Mombasa have always contested that Standard Swahili is a foreign language associated with Tanzania. They also claim that embracing it would lead to cultural domination because they regard Kimvita as their own cultural reservoir. The "ethnic neutrality" in question is also not viable because both the notions of "ethnic language" and "tribe" 
or "ethnic group" are problematic colonial constructs. So, claiming that Standard Swahili is not associated with any ethnic group is not enough to make it neutral and also does not guarantee that it will be accepted by the population at large. In fact, Kenyans who reject Standard Swahili do not do so on the grounds that it is associated with an ethnic group. Instead, my study found that they reject it for other reasons such as lack of prestige and lack of socioeconomic advantage.

At a broader level, the notions of "ethnic language" and "ethnic group" are problematic because they do not fit well in the analysis of the complex ethnolinguistic situation in Kenya and the rest of Africa. Instead, they were convenient social categories used by colonial administrators and missionaries to pursue their interests in Africa. Despite their problematic nature, I argued that they have become useful categories to the political ruling class in post-independence Kenya, especially with regard to matters of national interest such as general elections and distribution of national resources. This is partly why the official number of ethnic groups in Kenya has been contested, with scholars reporting figures as different as 42 and 70 .

The status of Standard Swahili as a national language has also been contested by speakers of Sheng. My interlocutors (including some, such as teachers, who do not regard themselves as Sheng speakers) claimed that it unites speakers from diverse ethnic backgrounds, a role that is officially assigned to Standard Swahili. They claimed that Sheng does so by incorporating linguistic material from various ethnic languages. Also, Sheng as an index of urbanity enables speakers to transcend ethnicity. This inference is consistent with the common claim among scholars of African urban youth vernaculars that the urban identity sought through the use of 
these vernaculars dissociates speakers from the rural settings, and older generations whom they associate with ethnicity.

Some speakers of Sheng envisioned it as the future national language of Kenya. While the exclusionary nature of Sheng contradicts their claim that Sheng has a unifying role, some of my findings indicate that Sheng is associated with a language ideology that seeks to unite people from diverse ethnic backgrounds, and at the same time maintain these differences. This challenges the notion of "homogeneism," associated with Standard Swahili, which seeks national unity by erasing ethnic differences (Blommaert and Verschueren 1998). Therefore, I conclude that the unifying language ideology of Sheng could be a solution to the perceived problem of multilingualism as a threat to national unity in Kenya and the rest of multilingual Africa. This conclusion, however, should not be misconstrued to mean that the government should officially recognize Sheng. Instead, I am suggesting that people can coexist with diverse ways of speaking. In fact, given that the unity of Sheng requires it to be subversive; its speakers would be opposed to the official adoption of Sheng for national use since it would lose its "beauty." Similary, the official recognition would be opposed by those young Kenyans such as speakers of lugha ya mtaa in Mombasa who do not afficliate with Sheng.

Even though speakers of lugha ya mtaa did not claim that it enabled them to transcend ethnicity, their characterization of Standard Swahili as "boring" and a language of low status further contributes to the contested status of Standard Swahili as the national language of Kenya. I recorded similar sentiments from speakers of Sheng in Nakuru who claimed that they avoided speaking Standard Swahili because it is hard, communicatively limiting and unromantic. Therefore, it is clear that many 
young Kenyans have a low regard for Standard Swahili and use it in very limited contexts. Interestingly, I found that many young Kenyans in Nakuru and those from the communities associated with the upcountry in Mombasa described the coastal Swahili accent as "sweet." Also, those from coastal communities who had visited Nairobi reported that people there admired their coastal Swahili accent. However, the various informants did not describe themselves as Waswahili for various reasons such as the negative stereotypes associated with that identity.

In an attempt to establish why Standard Swahili was not successful as the national language of Kenya, I drew comparisons with the neighboring Tanzania, where the language is more successful due to institutional and political support among other factors. However, it appears that English in both countries is the language of social mobility, hence people have a higher regard for English than Standard Swahili. In the Kenyan context, people are usually more worried about speaking English "correctly" than Standard Swahili. This is because many of these Kenyans, especially those working in the formal sector, still embrace colonial attitudes toward Swahili in general, associating it with people of lower social classes. Therefore, they perceive speaking Standard Swahili "correctly" as lowering their social status.

The dissertation also found that the dominant official language ideology in schools is paradoxical: though both Standard Swahili and English are official, school authorities regarded English as more official and important than Standard Swahili, with some teachers associating English with discipline, good education and intelligence. Such unequal treatment of the two official languages endows English with more prestige and power than Standard Swahili, perpetuating the colonial hegemonic status of English. This situation, however, is not limited to Kenya. Instead, 
it is a common phenomenon in post-colonial Africa, where despite standardizing local languages and even according them official status, the positive values associated with the former colonial languages such as English and French are not necessarily transferred to these standardized African languages (Adegbija 1994; Makalela 2005; Makoni et al., 2007).

Despite the strong emphasis in schools on the benefits of speaking English and Standard Swahili as "legitimate languages" (Bourdieu 1977), speakers of nonstandard varieties such as Sheng and lugha ya mtaa attach different values and meanings to the officially sanctioned languages. In some contexts, peers reprimand and ridicule those who use the two languages in peer conversations and also label them pejoratively. For example, while those who speak English are labeled as "show offs" and wasomi, those who speak Standard Swahili are labeled "washamba." Conversely, peers reward those who embrace styles such as Sheng, describing them as linguistically ingenious, culturally sophisticated, current, trendy, and fashionable and above all, people who have swag. In this context, I argued that there is a conflict in language ideology about the evaluation of language competence between school authorities and speakers of these stylistic forms such as Sheng. While school authorities reward the use of the standard language, speakers of Sheng and lugha ya mtaa reward the ability to innovate from various languages in one's repertoire. These speakers find other available languages, such as ethnic languages and the official languages inadequate for their communicative needs, and negotiating desired distinct social identities. I concluded that these unauthorized language varieties are alternative tools of communication accomplishing a subversive role among speakers. 
In both Nakuru and Mombasa, I observed that students came up with a counter-strategy against the use of the officially sanctioned languages. They reprimanded peers who spoke English and Swahili with an ethnic accent, among other causes of mispronunciation. They used the term "shrubbing" to refer to the various forms of mispronunciation. I employed the term "shrubbing discourse" for purposes of analysis to mean the act of highlighting or pointing out that a speaker has shrubbed. The discourse operates within a framework of witnesses (Irvine 2001) where peers maintain a tracking system for "shaming" and "faming" shrubbers.

While the shrubbing discourse seeks to discourage peers from speaking English and Swahili and to express disdain toward ethnic languages in favor of unauthorized language varieties such as Sheng, I argued that the discourse is paradoxical. On the one hand, though shrubbing is stigmatized among peers, not all shrubbers are reprimanded. Instead, if a "cool" guy shrubs, peers say that it is his or her swag. On the other hand, despite requiring speakers to demonstrate linguistic "correctness" in their use of English and Standard Swahili, peers do not reward those who speak the two languages "correctly." The discourse therefore discourages speakers from using the officially sanctioned English and Standard Swahili in favor of the unauthorized language varieties such as Sheng.

Speakers of Sheng and lugha ya mtaa also contravene identities socially ascribed within the local cultural frameworks and reinforced in schools such as masculinity and femininity. In both Nakuru and Mombasa, I observed that there were speakers of Sheng and lugha ya mtaa who contravened norms of formal speech by employing vulgarities or taboo expressions in their conversations. They also modified their school uniform in order to negotiate swag. The boys "piped" and "sagged" their 
pants and also tightened their sleeves to expose their biceps. The girls trimmed their skirts and tightened their sweaters to reveal their figures. While these are speakers' alternative masculinities and femininities respectively, they would not be approved of by the school authorities and other custodians of societal norms. Some teachers regarded these practices as markers of indiscipline and "indecency."

While the various social practices suggest that speakers embrace Sheng and lugha ya mtaa as indices of resistance, I observed that there are contexts when these speakers express conformant attitudes to the "standard language ideology," indicating that they operate in a "paradox of norms" (Kiessling and Mous 2004; Wairungu 2013). For example, some described English and Standard Swahili as "pure" languages and argued that people go to school to have their ethnic accent replaced with English. Interestingly, they associated English with education, intelligence and good career prospects, but not Standard Swahili. In this context, I concluded that while the notion of "standard language ideology" (Lippi-green 2012) fits to some extent for both English and Standard Swahili, the notion of "legitimate language" (Bourdieu 1977) does not fit well for Standard Swahili because its use generates social rewards in very limited contexts.

Speakers' embrace of these unauthorized language varieties has however been constantly fought by school authorities who seek to eradicate them. Nevertheless, given that students in Nakuru and Mombasa reported that they spoke these nonstandard language varieties most of the time, it is evident that the more schools fight them, the more they flourish. In this regard, I argued that there is a need for educators to adopt alternative approaches for addressing the presence of these nonstandard language varieties in schools because the current measures are 
counterproductive. Some possible measures may include utilizing them and associated cultural knowledge to facilitate the learning process both in the mastery of the standard language, and even in content subjects such as Mathematics. This recommendation is informed by some of my findings, for example in Nakuru, where some teachers reported that Sheng helped them reduce social distance while interacting with students. Also, I found that there were students who used Sheng productively, such as in peer teaching and academic discussion groups.

My research found that the society in general is developing positive attitudes toward nonstandard language varieties such as Sheng. First, I observed that not all teachers were opposed to Sheng, especially those in sports, religious organizations and guidance and counseling departments. Also, the Kenya Institute of Education has introduced a sociolinguistics section into the national Swahili curriculum aimed at promoting linguistic awareness among teachers and students. I collected samples of national exams with questions asking students about Sheng, and how it relates to other languages spoken within and outside the school. In addition, Sheng has spread to domains previously associated with Standard Swahili and English such as in the national television stations, where it is used to produce programs targeting young Kenyans.

Further, some politicians and religious leaders have strategically appropriated Sheng to reach out to young Kenyans. Even though such strategic appropriation may be motivated by socioeconomic and political interests, I argued that it is an indicator that the society in general has created more space to accommodate these nonstandard language varieties. My research findings may inform language planning in Kenya given that my study coincides with the recent call by the Minister of Education for a 
research study to establish whether and how Sheng affects students' performance in the officially sanctioned languages in schools.

Finally, the Sheng phenomenon is not peculiar to Kenya. Instead, there are other urban youth vernaculars such as Nouchi in Abidjan, Indoubil in Zaire and Town Bemba in Zambia with similar social and linguistic characteristics. The findings discussed in this dissertation would therefore inform any study focusing on such language varieties. Next, I focus on the limitations of the study.

\subsubsection{Limitations of the study}

I experienced several limitations during fieldwork, which potentially affected the success of this research project. Financially, I relied on personal savings to meet all my research expenses. As a result, I had to work with a very strict budget and live in residential neighborhoods with poor social amenities, e.g., unreliable water supply, electricity and security. The financial constraints also imposed time constraints, given that I aimed at conducting an intensive ethnographic study in two unfamiliar, geographically separated urban settings. I therefore had to do a lot within a very short period.

The other limitation was gaining the necessary acceptance either from individual informants, social groups or school authorities. Though I aimed at talking to as many informants as possible, there were some informants that I sought to interview or observe their classes but who did not honor my request. There were also some contexts where the various aspects of my identity as a researcher distracted informants thus affecting what they could say during our interactions. For example, being an outsider during a national teachers' strike, raised suspicion among some 
teachers that I might be a spy from the Ministry of Education who had come to investigate how they carried on their teaching. Also, my ethnic identity as a Kikuyu and being a former teacher of Standard Swahili could have affected how my informants perceived me. For example, I didn't have access to the Waswahili community in Mombasa, i.e. the main speakers of Kimvita, possibly because of my ethnic and regional identity. Recall Mr. Fujo's narrative about how coastal people use the term "Kikuyu" in protest when referring to the upcountry communities who own land and other economic resources at the coast. My religious identity as a Christian may also have affected my interactions with the coastal Swahili speakers since many of them, are Moslems. These social factors are partly why I did not record examples of lugha ya mtaa in Mombasa.

At the institutional level, I got rejected by the Principal of Rohoni School in Mombasa, which was the only school that matched the previous site in Nakuru from a demographic point of view. Despite these constraints, I was able to collect a rich set of data from the two research sites. Next, I focus on the potential areas for future research about Sheng and lugha ya mtaa.

\subsubsection{Directions for further research}

\subsubsection{Written forms of Sheng and conventions for their pronunciation}

In the course of my study in Nakuru and Mombasa, I encountered written forms of Sheng in magazines, advertisement posters and billboards, classroom walls, and comics such as Shujaaz. Some of these sources had a dictionary; a list of new words and their meanings, but none of them guided the reader on how to pronounce the sounds in the various forms of Sheng. It is important to investigate the 
pronunciation conventions of Sheng since it draws its linguistic material from various languages which have different conventions in spelling and pronunciation such as Dholuo, Kikuyu, Kiswahili and English. For example, the Sheng expression wamebeat 'they are old-fashioned' includes the English verb beat, which is pronounced with the English vowel/i/, even though Swahili spelling would dictate the pronunciation /ea/.

\subsubsection{Sheng and Hip Hop in Kenyan Schools}

In both Nakuru and Mombasa, I interacted with artistic informants, some of whom were known by the school authorties, who had embraced Hip Hop and rap music styles. Some of their songs were produced in Sheng. Though the majority wrote their lyrics in notebooks, there were some who had produced their music on Compact Disks (CDs), and sold it to peers. Some of these informants told me that they embraced music as a way of expressing their feelings, especially about the challenges they faced in life. Some of this music was gospel while the other was secular. Even though the administrations in both schools were opposed to such social practices, the Principal of Vikapuni School told me that she had allowed a particular student artist to produce and sell his music in order to raise money for his upkeep since he was an orphan from a financially challenged family. Outside the school, it would be important to investigate how students' appropriation of Sheng Hip Hop has been received in domains that are considered conservative, such as religious groups.

In Nakuru, for example, I asked Mr. Maragwa who described himself as a born-again Christian and sometimes engaged in preaching, to comment on the use of Sheng among young Kenyans in church. His response was: "Godliness has a standard. Godliness cannot be diffused with the world. What God has said, has said and you 
cannot water it down" (Mr. Maragwa 03/2011, casual conversation). In the same conversation, Mr. Maragwa said that Sheng and associated mode of dressing are indicators of an "open-ended mode of life," which he considered evil. However, in he used some Sheng his preaching, and when asked about this he replied that if he didn't use it, he would be unable to reach the youth, his target audience. An appropriate focus would be on the Akorino church because it is one of the religious groups in Kenya well known for its anti-secular doctrines. The choice of Akorino church is also inspired by the fact that I know of two young artists, Allan Aaron and Kamburi'z, who identify with this church and have produced gospel Hip Hop in Sheng, which has become very popular among many young Kenyans. ${ }^{90}$ The findings of such a study would contribute to the understanding of the uses and perception of Sheng in relation to other Kenyan languages, which was the main focus of the present research project.

\subsubsection{Grammatical features of lugha ya mtaa}

Future study should also investigate the grammatical features of lugha ya mtaa in Mombasa, given the earlier claim that the matrix language of Sheng is usually the local variety of Swahili. This would provide more evidence about how Sheng differs from lugha ya mtaa besides vocabulary and social factors such as speakers' attitudes. It would also show the relationship between lugha ya mtaa and local varieties of Swahili in Mombasa.

\footnotetext{
${ }^{90}$ Some of their popular songs are Kiriro 'http://www.youtube.com/watch?v=V9HpwJgXwqE' and Christian Party ‘http://www.youtube.com/watch?v=49XASIK3U1U’
} 


\section{Bibliography}

Abdul-Aziz, Mohamed (1979). Muyaka, 19th century Swahili popular verse. Nairobi: Kenya Literature Bureau.

Abdul-Aziz, Mohamed, and Osinde, Kenneth (1997). Sheng and Engsh: development of mixed codes among urban youths in Kenya. International Journal of the Sociology of Language 125:45-63.

Adegbija, Efurosibina (1994). Language attitudes in Sub-saharan Africa. Clevedon, UK: Multilingual Matters.

Ahearn, Laura (2012). Living language: an introduction to Linguistic Anthropology. Wiley-Blackwell.

Alim, Samy (2004). Hip Hop Nation Language. In Edward Finegan and John Rickford (eds.), Language in the USA: themes for the $21^{\text {st }}$ century. Cambridge: Cambridge University Press, pp. 387-409.

Argenti, Nicolas (2007). The intestines of the state: youth, violence, and belated histories in the Cameroon grass fields. U.S.A: Chicago University Press.

Bakari, Mohammed (1985). The morphology of the Kenyan Swahili dialects. Berlin: Dietrich Reimer Verlag.

Bakhtin, Mikhail (1968). Rabelais and his world. Translated by H. Iswolsky. Cambridge, Massachusetts: MIT Press.

(1982). The dialogic imagination: four essays. Austin: University of Texas Press.

Batibo, Herman (1995). The growth of Kiswahili as language of education and administration in Tanzania. In Martin Putz (ed.), Discrimination through language in Africa: perspectives on the Namibian experience. New York: Mouton de Gruyter, pp. 57-80.

Bereiter, Carl, and Engelmann, Siegfried (1966). Teaching disadvantaged children in the preschool. Englewood Cliffs, NJ: Prentice-Hall.

Biernacki, Patrick, and Waldorf, Dan (1981). Snowball sampling: problem and techniques of chain referral sampling. Sociological methods and research 10(2):141-163.

Bleek, Immanuel (1869). A comparative grammar of South African languages. Part 
II: The concord; section I: the noun. Cape Town and London: J.C. Juta and Trübner \& Co.

Blommaert, Jan, and Verschuerenv, Jef (1998). The role of language in European nationalist ideologies. In Bambi Schieffelin, KathrynWoolard and Paul Kroskrity (eds.), Language ideologies: practice and theory. Oxford: Oxford University Press, pp. 189-210.

Bosire, Mokaya (2006). Hybrid languages: the case of Sheng. In Arasanyin Olaoba and Michael Pemberton (eds.), Selected proceedings of the 36th annual conference on African linguistics. Somerville: Cascadilla Proceedings Project, pp. 185-193.

(2008). Sheng: the phonology, morphology and social profile of an urban vernacular. Unpublished Dissertation. State University of New York at Albany.

Bourdieu, Pierre (1977). The economics of linguistic exchanges. Social science information 16(6):645-668.

BROCK-UTNE, Birgit (2005). The continued battle over Kiswahili as the language of instruction in Tanzania. In Brock-Utne Birgit and Rodney Hopson (eds.), Languages of instruction for African emancipation: focus on postcolonial contexts and considerations. Cape Town: CASAS and Dar es Salaam: Mkuki na Nyota, pp. 51-88.

Brown, Rap (1972). Street talk. In Thomas Kochman (ed.), Rappin' and stylin' out. Urbana: University of Illinois Press, pp. 205-208.

Bucholtz, Mary (1999). "Why be normal?": Language and identity practices in a community of nerd girls. Language in Society 28:203-223.

Bunyi, Grace (2005). Language classroom practices in Kenya. In Angel Lin and Peter, Martin (eds.), Decolonisation, globalisation: language-in-education policy and practice. Clevedon, England: Multilingual Matters, pp. 133-154.

Buwembo, Joachim (April 26 ${ }^{\text {th }}$ 2013). Uganda: how Kiswahili stopped being the language of soldiers and prostitutes. Africa Review. Retrieved August $15^{\text {th }}$ 2013, from < http://www.africareview.com/Special-Reports/Why-Kiswahilihas-taken-off-in-Uganda/-/979182/1760180/-/afgg54/-/index.html>

Bwenge, Charles (2002). Codeswitching in political discourse in Tanzania: a case study of parliamentary proceedings. Unpublished doctoral dissertation. University of Virginia.

Cameron, Deborah (1995). Verbal hygiene. London: Routledge. 
Chachage, Chambi (April 29 ${ }^{\text {th }} 2010$ ). Africa: there's No Such Thing as Plain Kiswahili. Pambazuka News: comment and Analysis. Retrieved October $13^{\text {th }}$ 2013, from http://www.pambazuka.org/en/category/comment/64058>

Cheshire, Jenny (1998). The relationship between language and sex in English. In Michael, Linn (ed.), Handbook of dialects and language variation. San Diego, California: Academic Press, pp. 403-418.

Chimerah, Rocha (1998). Kiswahili: past, present and future horizons. Nairobi: Nairobi University Press.

Chiraghdin, Shihabuddin and Mnyampala, Mathias (1977). Historia ya Kiswahili. Nairobi: Oxford University Press.

Contini-Morava, Ellen (2000). Noun class as number in Swahili. In Ellen Contini Morava and Tobin Yishai (eds.), Between lexicon and grammar. Amsterdam: John Benjamins, pp. 3-30.

Coup, Da Pidgin (2008). Pidgin and education: a position paper. Journal of the College of Education 41(1 \& 2): 55-76.

Coupland, Nikolas (2007). Style: language variation and identity. Cambridge: Cambridge University Press.

Dave, Opiyo, and Walter, Menya (October $17^{\text {th }}$ 2013). Ruto asked Kalenjins to evict Kikuyus at rally, says witness. Daily Nation Newspaper. Retrieved July $12^{\text {th }}$ 2013, from <http://www.nation.co.ke/news/politics/Ruto-asked-Kalenjins-toevict-Kikuyus-at-rally-says-witness/-/1064/2037470/-/5ssa1q/-/index.html>

Deen, Kamil (2002). The acquisition of Nairobi Swahili: the morphosyntax of inflectional prefixes and subjects. Unpublished Doctoral dissertation. University of California, Los Angeles.

DE Meulder, Bruno (1998). Nakuru, a patchwork. From colonial outpost to East Africa town. In Bruno De Meulder, André Loeck and Susan Shannon (eds.), Nakuru: an African town. Leuven: City of Leuve/Catholic University, pp. 1334.

Derwing, Tracey, and Munro, Murray (2009). Putting accent in its place: rethinking obstacles to communication. Language teaching 42 (4): 476-490.

Eastman, Carol (1971). Who are the Waswahili? Africa 41(3): 228-36.

(1984). Modern Swahili: is there a generation gap? In Joan Maw and David Parkin (eds.), Swahili language and society. Vienna:

Beitrage zur Afrikanistik, pp. 297-318. 
Eckert, Penelope (1989). Jocks and burnouts: Social categories and identity in the high school. New York: Teachers College Press.

(2008). Variation and the indexical field. Journal of Sociolinguistics 12(4): 453-476.

Eckert, Penelope, and McConnell-Ginet, Sally (1992). Communities of practice: where language, gender and power all live. In Hall Kira, Mary Bucholtz and Birch Moonwomon (eds.), Locating power: proceedings of the second Berkeley women and language conference. Berkeley, CA: Berkeley Women and Language Group, pp.89-99.

(2013). Language and gender (second edition). New York: Cambridge University press.

Eshiwani, George (1993). Education in Kenya since independence. Nairobi: East African Educational Publishers.

Fabian, Johannes (1991). Language and colonial power: the appropriation of Swahili in the former Belgian Congo1880-1938. Berkeley: University of California Press.

Fischer, John (1972). The stylistic significance of consonantal sandhi in Trukese and Ponapean. In John Gumperz and Dell Hymes (eds.), Directions in sociolinguistics. New York: Holt, Rinehart and Winston, pp. 498-511.

Foeken, Dick (2006). To subsidise my income: urban farming in an East-African town. Leiden: Brill Academic Publishers.

Freedman, Ariella, and Todd, Judith (1994). Kenyan women tell a story: interpersonal power of women in three subcultures in Kenya. Sex Roles 31(9/10):533-546.

Gal, Susan, and Irvine, Judith (1995). The boundaries of languages and disciplines: how ideologies construct difference. Social research 62:967-1001.

Githinji, Peter (2006). Sheng and variation: the construction and negotiation of multiple identities. Unpublished doctoral dissertation. Michigan State University.

(2006). Bazes and their shibboleths: lexical variation and Sheng speakers' identity in Nairobi. Nordic Journal of African Studies 15(4):443-472.

(2008). Ambivalent attitudes: perception of Sheng and its speakers. Nordic Journal of African Studies 17(2):113-136.

Githiora, Chege (2002). Sheng: peer language, Swahili dialect or emerging creole? Journal of African Cultural Studies 15:159-181. 
Given, Michael (2004). The archaeology of the colonized. London: Routledge.

Goffman, Erving (1959). The presentation of self in everyday life. Garden City, NY: Doubleday.

Gorman, Patrick (1974). The development of language policy in Kenya with particular reference to the educational system. In Wiliam Whiteley (ed.), Language in Kenya. Nairobi: Oxford University Press, pp. 397-453.

Gosden, Chris (2004). Archaeology and colonialism: cultural contact from 5000 BC to the present. U.K: Cambridge University Press.

Gumperz, John (1982). Discourse strategies. Cambridge: Cambridge University Press.

Halliday, Craig (June 12 $2^{\text {th }}$ 2012). Sheng: language of Empowerment or Disobedience? Think Africa Press. Retrieved August $14^{\text {th }} 2013$, from <http://thinkafricapress.com/kenya/sheng-language-empowerment-ordisobedience>

Halliday, Michael (1976). Anti-languages. American anthropologist 78:570-84.

Handler, Richard (1994). Is 'identity' a useful cross-cultural concept? In John Gillis (ed.), Commemorations: the politics of national identity. Princeton: Princeton University Press, pp. 27-40.

Haugen, Einar (1972). Dialect, language and nation. In John Pride and Janet Holmes (eds.), Sociolinguistics: selected readings. London: Penguin, pp.97-111.

Hill, Jane (1998). Language, race, and white public space. American anthropologist 100(3):680-689.

Hinnebusch, Thomas, and Mirza, Sarah (1998). Kiswahili: msingi wa kusema, kusoma, na kuandika (second edition). Maryland: University Press of America.

Holt, Grace (1972). 'Inversion' in black communication. In Kochman Thomas (ed.), Rappin' and stylin' out: communication in urban black America. Urbana, IL: University of Illinois Press, pp.152-159.

Horton, Mark, and Middleton, John (2000). The Swahili: the social landscape of a mercantile society. U.S.A: Blackwell publishers.

Hymes, Dell (1968). Linguistic problems in defining the concept of 'tribe'. In June Helm (ed.), Essays on the problem of tribe. Seattle: University of Washington Press, pp. 23-48. 
Insoll, Timothy (2003). The archaeology of Islam in Sub-Saharan Africa. Cambridge: Cambridge University Press.

Irvine, Judith (1979). Formality and informality in communicative events. American Anthropologist 81(4):773-790.

(2001). "Style" as distinctiveness: the culture and ideology of linguistic differentiation. In Penelope Eckert and John, Rickford (eds.), Style and sociolinguistic variation. Cambridge: Cambridge University Press, pp. 21-43.

Jaffe, Alexandra (2009). Stance: sociolinguistic perspectives. New York: Oxford University Press.

Jagero, Juliet, and Odongo, Eston (2012). Manifestation of formal and informal codeswitching in Nairobi, Kenya. Academic research international. SAVAP 2(2):364-372.

Kagwanja, Peter (2003). Facing Mount Kenya or facing Mecca? The Mungiki, ethnic violence and the politics of the Moi succession in Kenya, 1987-2002. African affairs 102:25-49.

Kanana, E. (2003). Codeswitching in business transactions: a case study of repertoire in Maasai Market in Nairobi, Kenya. Unpublished M.A. thesis. Kenyatta University.

Kang'ethe-Iraki, Fredrick (2004). Cognitive efficiency: the Sheng phenomenon in Kenya. Pragmatics. International pragmatics association, 14(1):55-68.

(2010). Language and political economy: a historical perspective from Kenya. TRANS, 17:1-3.

Kawoya, Vin (1984). Kiswahili in Uganda. In Joan Maw and David Parkin (eds.), Swahili language and society: papers from the workshop held at the School of Oriental and African Studies in April 1982. Wien: Afro-Pub, pp. 3545 .

Khalidi, Abdalla (1977). The liberation of Swahili from European appropriation. Nairobi: Kenya Literature Bureau.

Kiessling, Roland, and Mous, Maarten (2004). Urban youth languages in Africa. Anthropological Linguistics 46 (3):303-341.

Kisaalita, William, and Sentongo-Kibalama, Josephat (2007). Delivery of urban transport in developing countries: the case for the motorcycle taxi service (bodaboda) operators of Kampala. Development Southern Africa 24:345 -357 . 
Kitsao, Jay (December $11^{\text {th }}$ 1977). Time to make Swahili truly a national language. Nairobi Times.

Kitula, King'ei (2001). Pitfalls in Kenya's postcolonial language policy: ambivalence in choice and development. Per Linguam 17(1):36-47.

Klopp, Jacqueline (2001). Can moral ethnicity trump political tribalism? The struggle for land and nation in Kenya. African Studies 61(2):269-294.

Kochman, Thomas (1981). Black and white styles of conflict. Chicago and London: University of Chicago Press.

Labov, William (1966). The social stratification of English in New York city. Washington, DC: Center for Applied Linguistics.

(1972). The logic of nonstandard English. In Frederick Williams (ed.), Language and poverty: perspectives on a theme. Chicago: Markham, pp. 153-189.

Leichty, Mark (1995). Modernization, media, and markets: youth identities and the experience of modernity in Kathmandu, Nepal. In Vered Amit-Talia and

Lin, Angel (2009). "Respect for da chopstick Hip Hop": the politics, poetics, and pedagogy of Cantonese verbal art in Hong Kong. In Samy Alim, Awad Ibrahim and Alastair Pennycook (eds.), Global linguistic flows: Hip Hop cultures, identities, and the politics of language. Mahwah, New Jersey: Lawrence Erlbaum, pp. 159-177.

Lippi-Green, Rosina (2012). English with an accent: language, ideology, and discrimination in the United States. London: Routledge.

Loren, DiPaolo (2008). In contact: bodies and spaces in the sixteenth-and seventeenth-century Eastern woodlands. Lanham, MD: Altamira Press.

Lyndon, Harries (1976). The nationalization of Swahili in Kenya. Language in society. Cambridge University Press, 5:153-164.

Makalela, Leketi (2005). We speak eleven tongues: reconstructing multilingualism in South Africa. In Birgit Brock-Utne and Rodney Kofi Hopson (eds.), Languages of instruction for African emancipation: focus on postcolonial contexts and considerations. Dar es Salaam: Mkuki and Nyota Publishers, pp. 147-175.

Makoni, Sinfree, Brutt-Griffler, Janina, and Mashiri, Pedzisai (2007). The use of 'indigenous' and urban vernaculars in Zimbabwe. Language in Society 36 (1): $25-49$. 
Mazrui, Alamin (1995). Slang and code-switching: the case of Sheng in Kenya. Afrikanistiche Arbeitspapiere 42:168-179.

Mazrui, Ali and Mazrui, Alamin (1995). Swahili, state and society: the political economy of an African language. Nairobi: East African Educational Publishers.

(1996). A tale of two Englishes: the imperial language in the post-colonial Kenya and Uganda. In Joshua Fishman, Andrew Conrad and Rubal-Lopez Alma (eds.), Post Imperial English. Berlin: Mouton de Gruyter, pp. 271-302.

Mbaabu, Ireri (1985). New horizons in Kiswahili. Nairobi: Kenya Literature Bureau.

McLaughlin, Fiona (2009). Introduction to the languages of urban Africa. In Fiona Mc Laughlin (ed.), The languages of urban Africa. New York: Continuum International Publishing Group, pp. 1-19.

Migunda-Attyang, Jane (2007). Attitudes toward Sheng: an emerging language in Nairobi. Chemichemi: International Journal of the School of Social Sciences. Nairobi: Kenyatta University, 4 (1):29-43.

Mkangi, Katama (1984). The political economy of Kiswahili. A Kenya: Tanzania comparison. In Joan Maw, David Parkin (eds.), Swahili language and society. Vienna: Beitrage zur Afrikanistik, pp. 331-345.

Mkude, Daniel (1984). The fate of Standard Swahili. In Joan Maw and David Parkin (eds.), Swahili language and society. Vienna: Beitrage zur Afrikanistik, pp. 25-34.

Momanyi, Clara (2009). The effects of Sheng in the teaching of Kiswahili in Kenyan schools. The Journal of Pan African Studies 2(8):127-138.

Mugane, John (2005). Necrolinguistics: linguistic-death-in-life. The Du Bois Review $2(2): 159-186$.

Muigai, Chege (February $21^{\text {st }}$ 2012). Sheng "will be one of the most dominant languages in 2050." Daily Nation Newspaper. Retrieved August $15^{\text {th }} 2013$, from<http://www.nation.co.ke/Features/DN2/Sheng+will+be+one+of+the+mo $\underline{\text { st+dominant+languages+in+2050+/-/957860/1331330/-/yntys/-/index.html }>}$

Mungai, Christine (January $17^{\text {th }}$ 2011). Kiswahili's future lies in borrowing from English. The East African Magazine. Retrieved June $10^{\text {th }} 2013$, from <http://www.theeastafrican.co.ke/magazine/Kiswahilis-future-lies-inborrowing-from-English/-/434746/1088936/-/i2srou/-/index.html>

Murunga, Godwin, and Nasong'o, Shadrack (2007). Kenya: the struggle for democracy. Dakar, Senegal: CODESRIA Books. 
Mutahi, Karega (1979). Contradictions between language policy and language practice in Kenya. Staff Seminar Paper. University of Nairobi, 4:1-11.

Mutiga, Jayne (2013). Effects of language spread on a people phenomenology: the case of Sheng in Kenya. Journal of Language, Technology and Entrepreneurship in Africa. Nairobi, 4 (1):1-14.

Mwangi, Samson (2002). Challenges of urban environmental governance: participation and partnerships in Nakuru municipality, Kenya. Amsterdam: AGIDS.

Mwiria, Kilemi (1991). Education for subordination: African education in colonial Kenya. Kenyatta University: Bureau of Educational Research 20 (3): 261-273.

Myers-Scotton, Carol (1993). Social motivations for code-switching: evidence from Africa. Oxford: Clarendon Press.

Nabea, Wendo (2009). Language policy in Kenya: negotiation with hegemony. The Journal of Pan African Studies 3(1):121-138.

Nadel, Frederick (1947). The Nuba: an anthropological study of the hill tribes in Kordofan. London: Oxford University Press.

Newell, Sasha (2009). Enregistering modernity, bluffing criminality: how Nouchi speech reinvented (and fractured) the nation. Journal of Linguistic Anthropology 19 (2):157-184.

Nurse, Derek, and Hinnebusch, Thomas (1993). The Swahili and Sabaki: a linguistic history. Berkeley, California: University of California Press.

Ochieng, Philip (September $21^{\text {st }} 2013$ ). Wanted: Inquiry into what ails language Teaching. Daily Nation Newspaper. Retrieved August $15^{\text {th }} 2013$, from < http://mobile.nation.co.ke/blogs/Wanted-Inquiry-into-what-ailslanguage-teaching-/-/1949942/2001990/-/format/xhtml/-/vyamcw/lindex.html>

Ociti, Peter (1973). African indigenous education. Nairobi, Kenya: East-African Literature Bureau.

Ogechi, Nathan (2002). Trilingual codeswitching in Kenya; evidence from Ekegusii, Kiswahili, English and Sheng. Unpublished dissertation. Hamburg: University of Hamburg. (2005). On lexicalization in Sheng. Nordic Journal of African Studies 14(3):334-355.

Olick, Felix. (October $24^{\text {th }} 2013$ ). Witness tell ICC judges attackers 
asked coded questions to isolate victims. Standard Newspaper. Retrieved $15^{\text {th }}$ June 2013, from

$<$ https://www.standardmedia.co.ke/mobile/?articleID=2000096098\&story_titl $e=$ icc-witness-recalls-how-youths-attacked-kikuyus>

Ominde, Simeon (1964). Kenya education commission report. Nairobi: Government Printer.

Orengo, Peter, and Opiyo, Peter (December $29^{\text {th }}$ 2011). Sheng affecting English, Kiswahili performance. Standard Newspaper. Retrieved July $10^{\text {th }} 2013$, from <http://www.standardmedia.co.ke/?articleID=2000049107\&pageNo=1 >

Owuor, Samuel (2006). Bridging the urban-rural divide: multi-spatial livelihoods in Nakuru town, Kenya. ASC research report. Leiden: African Studies Centre.

Papastergiadis, Nikos (1997). Tracing hybridity in theory. In Pnina Werbner and Tariq Modood (eds.), Debating cultural hybridity: multi-cultural identities and the politics of anti-racism. London, Zed Books, pp. 257-281.

Powers, Sherry (2002). Real ways of talking and school talking: one Appalachian student's perception of teacher discourse during writing conferences. Reading Horizons 43(2):85-102.

Pullum, Geoffrey (1999). African American vernacular English is not Standard English with mistakes. In Wheeler Rebecca (ed.), The workings of language: from prescriptions to perspectives. Westport, Connecticut, and London: Praeger, pp. 39-58.

Puttenham, George (1589). The arte of English poesie. In Gregory, Smith (ed.), Elizabeth Critical Essays. Oxford: Clarendon Press. Vol. 2.

Richardson, Irvine (1964). Linguistic change in Africa with special reference to the Bemba-speaking area of Northern Rhodesia. Symposium on multilingualism. London: Commission for Technical Co-operation in Africa, 189-196.

Rickford, John (1999). The Ebonics controversy in my backyard: a sociolinguist's experiences and reflections. Journal of Sociolinguistics 3: 267-275.

Rudd, Philip (2008). Sheng: The mixed language of Nairobi. Unpublished doctoral Dissertation. Ball State University.

Sabrina, Billings (2009). Speaking beauties. Language in Society. Cambridge University Press, 581-606.

Samper, David (2002). Talking Sheng: the role of a hybrid language in the 
construction of identity and youth culture in Nairobi, Kenya. Unpublished doctoral dissertation. University of Pennsylvania.

Shiundu, Alphonce (June $8^{\text {th }}$ 2011). MPs move to ban vernacular in public offices Daily Nation Newspaper. Retrieved August $15^{\text {th }} 2013$, from $<$ http://www.nation.co.ke/news/politics/MPs-move-to-ban-vernacular-inpublic-offices-/-/1064/1177070/-/fxay3v/-/index.html>

Siegel, Jeff (1999). Stigmatized and standardized varieties in the classroom: Interference or separation? Teachers of English to speakers of other languages, Inc. Tesol Quarterly 33(4):701-728.

(2008). Pidgin in the classroom. Journal of the College of Education, University of Hawai' $i$ at Manoa 41(1\&2):55-76.

Sifuna, David (1990). Development of education in Africa: the Kenyan experience. Nairobi: Initiatives Publishers Ltd.

Silverstein, Michael (1979). Language structure and linguistic ideology. In Clyne Paul, Hanks William and Charles Hofbauer (eds.), The elements: a parasession on linguistic units and levels. Chicago: Chicago Linguistic Society, pp. 193-247.

Singh, Holly (2011). Aulad: Infertility and the meanings of children in North India. Unpublished doctoral dissertation.University of Virginia.

Snoxall, Ronald (1984). The east African interterritorial language (Swahili) committee. In Joan Maw and David Parkin (eds.), Swahili language and society. Vienna: Beitrage zur Afrikanistik, pp. 15-24.

Spitulnik, Deborah (1999). The language of the city: Town Bemba as urban hybridity. The Journal of Linguistic Anthropology 8 (1):30-59.

Spyropoulos, Mary (1987). Sheng: some preliminary investigations into a recently emerged Nairobi Street Language. Journal of Anthropological Society of Oxford 18(1):125-136.

The Government of Kenya (2009). Population and housing census report. Ministry of state for planning, national development and vision 2030. Nairobi, Kenya: Government Printers.

(2010). The National Constitution. Nairobi: Government printers.

Thomason, Sarah (2001). Language contact. Edinburgh: Edinburgh university press.

Thompson, Katrina (2010). "I am Maasai": interpreting ethnic parody in Bongo

Flava. Language in Society 39 (4): 493-520. 
Thuku, Wahome (August $30^{\text {th }}$ 2010). Kiswahili becomes Kenya official language. Standard Newspaper. Retrieved July $17^{\text {th }} 2013$, from $<$ http://www.standardmedia.co.ke/?articleID=2000017118\&pageNo=1\&story title=Kiswahili-becomes-Kenya-official-language $>$

Trudgill, Peter (1974). The social differentiation of English in Norwich. Cambridge: Cambridge University Press.

Urciuoli, Bonnie (1996). Exposing prejudice. Boulder: Westview Press.

Wairungu, Michael (2013). The rise of Sheng: a sociolinguistic revolution from below. In Vivian Yenika-Agbaw and Lindah Mhando (eds.), African youth in contemporary culture: Identity quest. New York:

Routledge, pp. 164-183.

Waki, Paul (2008). Government of Kenya, report of the commission of inquiry into post-election violence (CIPEV - The Waki commission). Kenya: Nairobi, 2008.

Wald, Benji (1973). Variation in the system of tense markers of Mombasa Swahili. Unpublished doctoral dissertation. Columbia University.

(1985). Vernacular and Standard Swahili as seen by members of the Mombasa Swahili speech community. In Wolfson Nessa and Joans Manes (eds.), Language of inequality. Berlin and New York: Mouton, pp. 123-143.

Wallace, Claire, and Sijka, Kovatcheva (1998). Youth in society: the construction and deconstruction of youth in East and West Europe. London: Macmillan Press Ltd.

Wa'Njoroge, Kimani (1985). Language in education in Africa: proceedings of a seminar held in the center of African studies, University of Edinburgh, $29^{\text {th }}$ and $30^{\text {th }}$ November. Centre of African Studies. University of Edinburgh.

Wa' Thiong'o, Ngugi (1986). Decolonising the mind. Nairobi: Heinemann.

Whiteley, Wilfred (ed.), (1974). Language in Kenya. Nairobi: Oxford University Press.

(1993). Swahili: the rise of a national language. London:Methuen.

Zuengler, Jane (1985). English, Swahili, or other languages? The relationship of educational development goals to language of instruction in Kenya and Tanzania. In Wolfson Nessa and Joans Manes (eds.), Language of inequality. Berlin and New York: Mouton, pp. 241-54. 


\section{Appendices}

\section{Appendix A: Sample print material used for eliciting comments}

\section{nember}

- friends or anyone else should not sure you into doing drugs.

zs won't help you have fun, be spted or feel grown up.

js are illegal.

js will ruin your education, your ionships with your friends and ly.

js will stop you from working urds your dreams and aspirations.

\section{Wakisay,} reryone is doing t..." Washow, $n$ not everyone, I'm myself!"

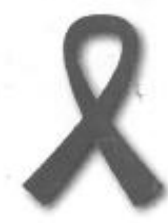

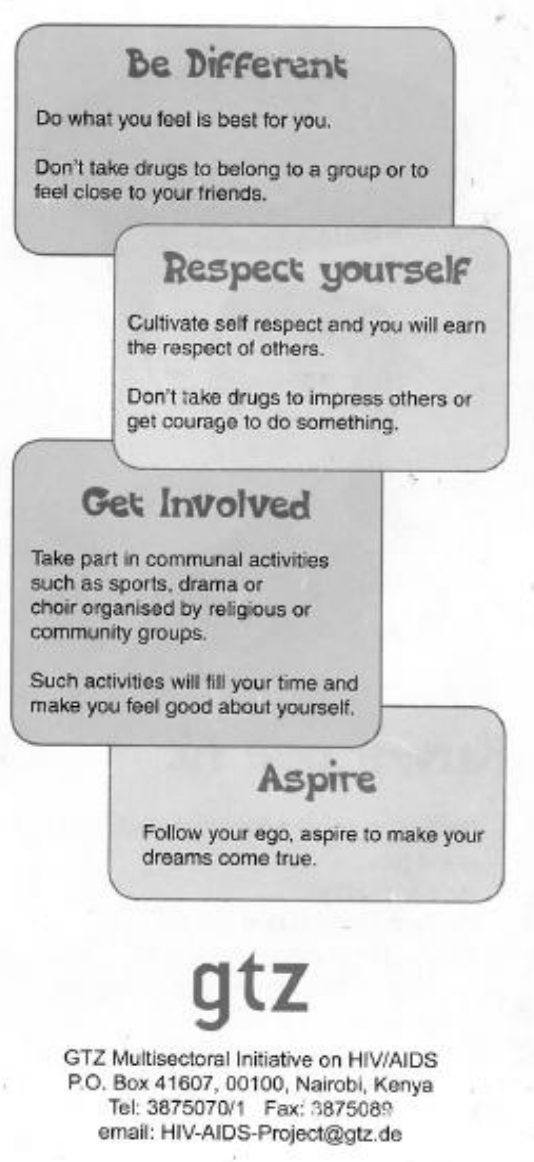

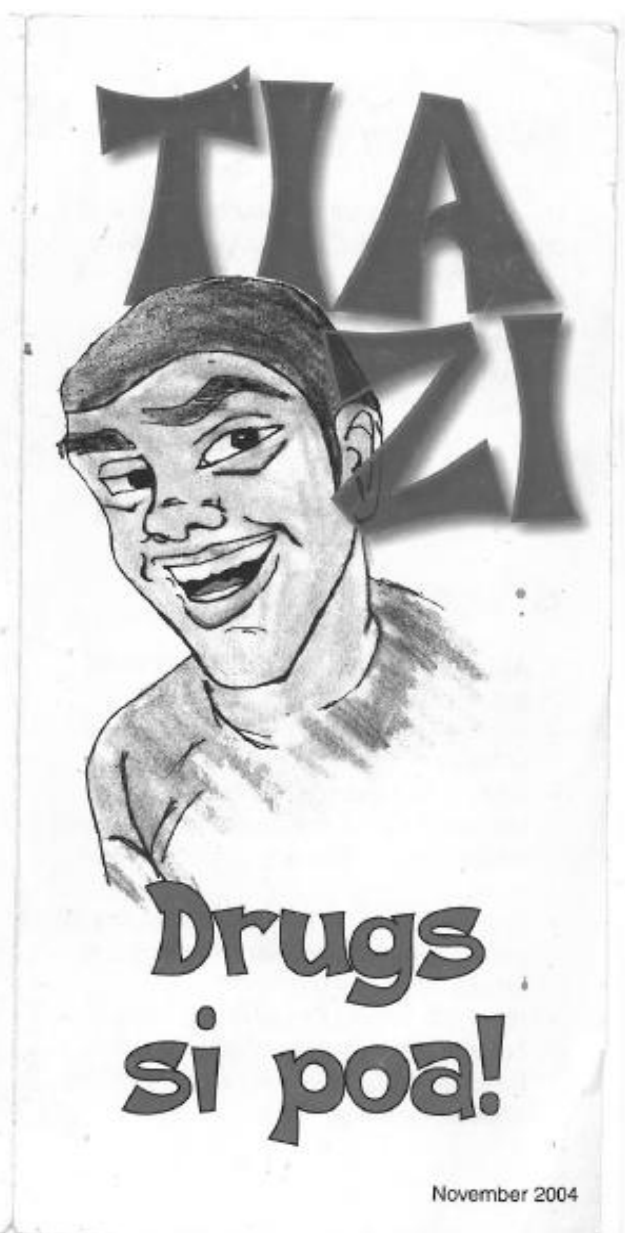




\section{Appendix B: Sample print material used for eliciting comments.}

\section{What are drugs?}

Drugs are chemical substances that can change your mood, your understanding or your behaviour.

The drugs we take when we are sick are medicine and are meant to heal our bodies.

\section{Commonly abused drugs in Kenya}

- ALCOHOL-especially locally brewed e.g. kumikumi. Alcohol is equally consumed by adult men, women and youth.

- CANNABIS-[known as BHANG or MARIJUANA] is the most common illegal drug traditionally produced and abused.

- KHAT-[known as MIRAA] especially in the East African highlands where it is produced.

- Other dangerous illegal drugs such as cocaine, heroin, pills, methaqualone [also known as madrax] are commonly abused by urban youth.

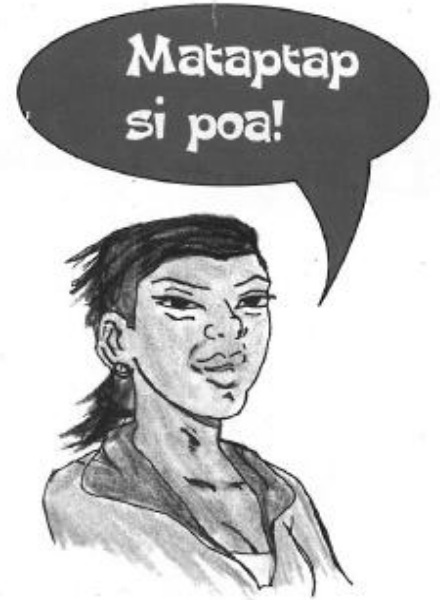

\section{Kuwa poa ni:}

- Getting a good education and having a good job.

- Being healthy.

- Being a good role model.

- Staying in control of your life.

- Getting professional counselling to

help you quit the habit.

- Being in a good relationship with your family, friends and community.
Tia Zi! Be differe and say "NO" to drugs.

- Drugs will destroy your future, the make you live for "NOW".

- If you are taking drugs, the truth $i$ you are no longer in control of you life. Your addiction to the drugs ar controlling you!

- Drugs work directly on your brain, damaging it or even causing deatl

- Drugs lure you into irresponsible behaviour like casual and unprote sex-which exposes you to Sexual Transmitted Infections [STIs] and HIV/AIDS, rape, criminal activities dropping out of school.

- Drugs will damage your personal health. 
Appendix C: Modified school uniform to negotiate swag



Appendix D: Nonlinguistic swag outside school

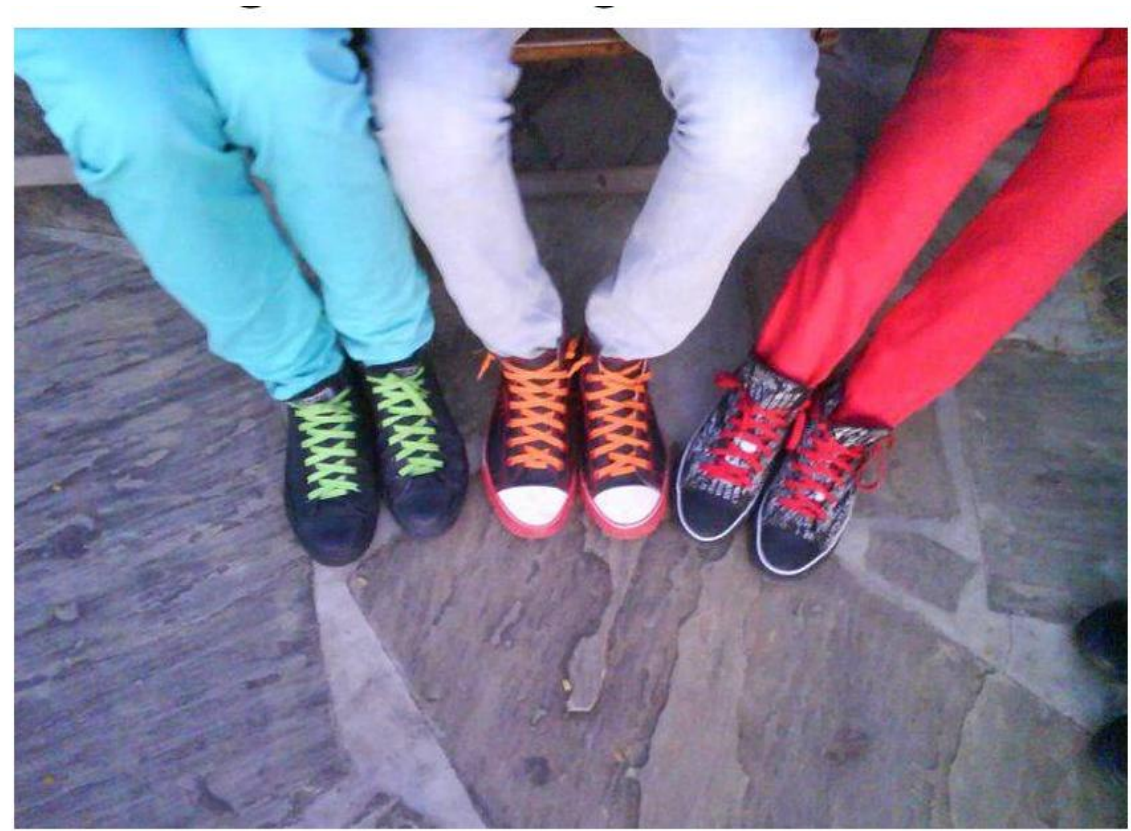


Appendix E: The revised Vikapuni School language policy

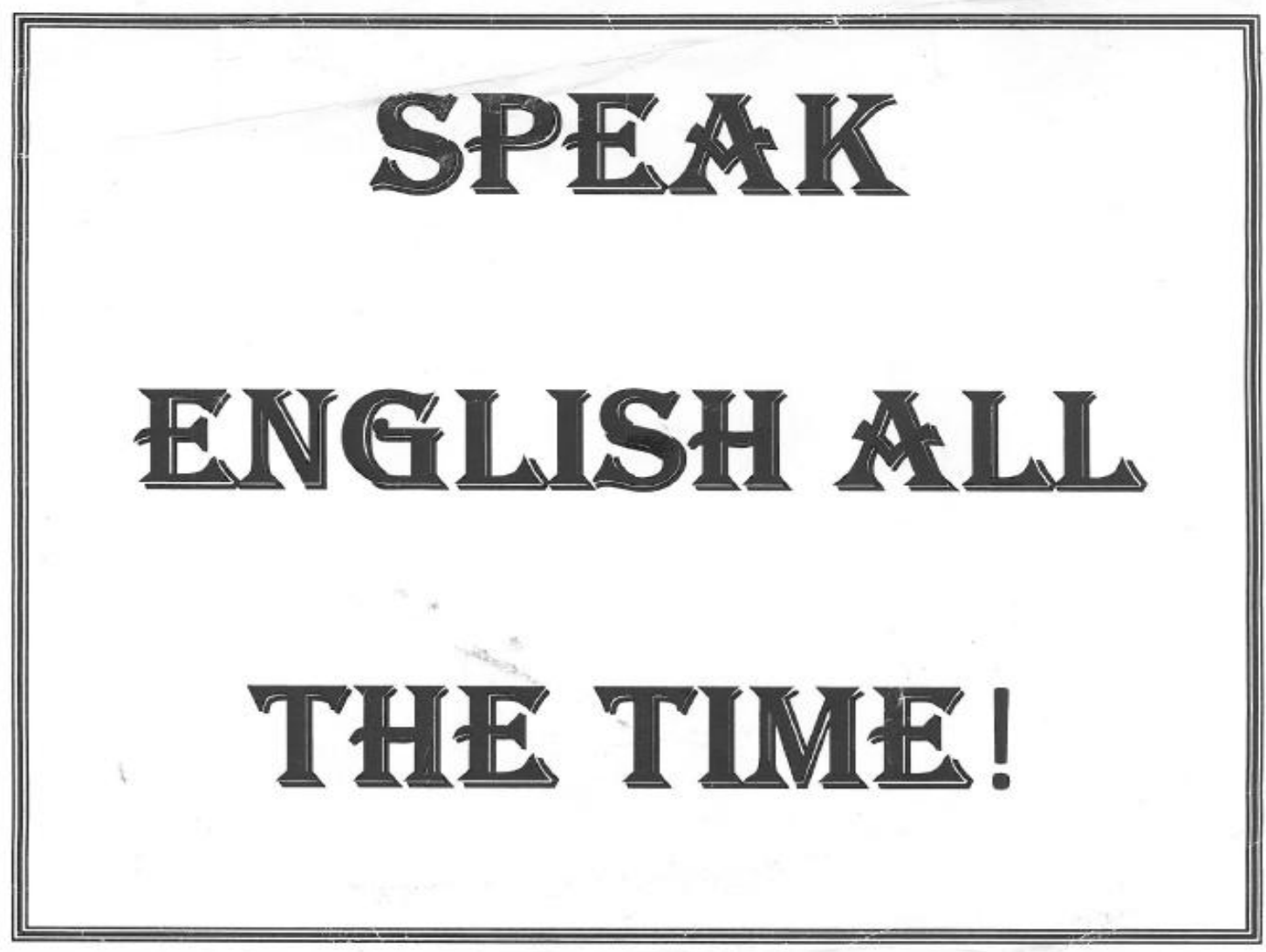


Appendix F: Research sites

Map of Kenya Showing the Geographical Location of Nakuru and Mombasa.

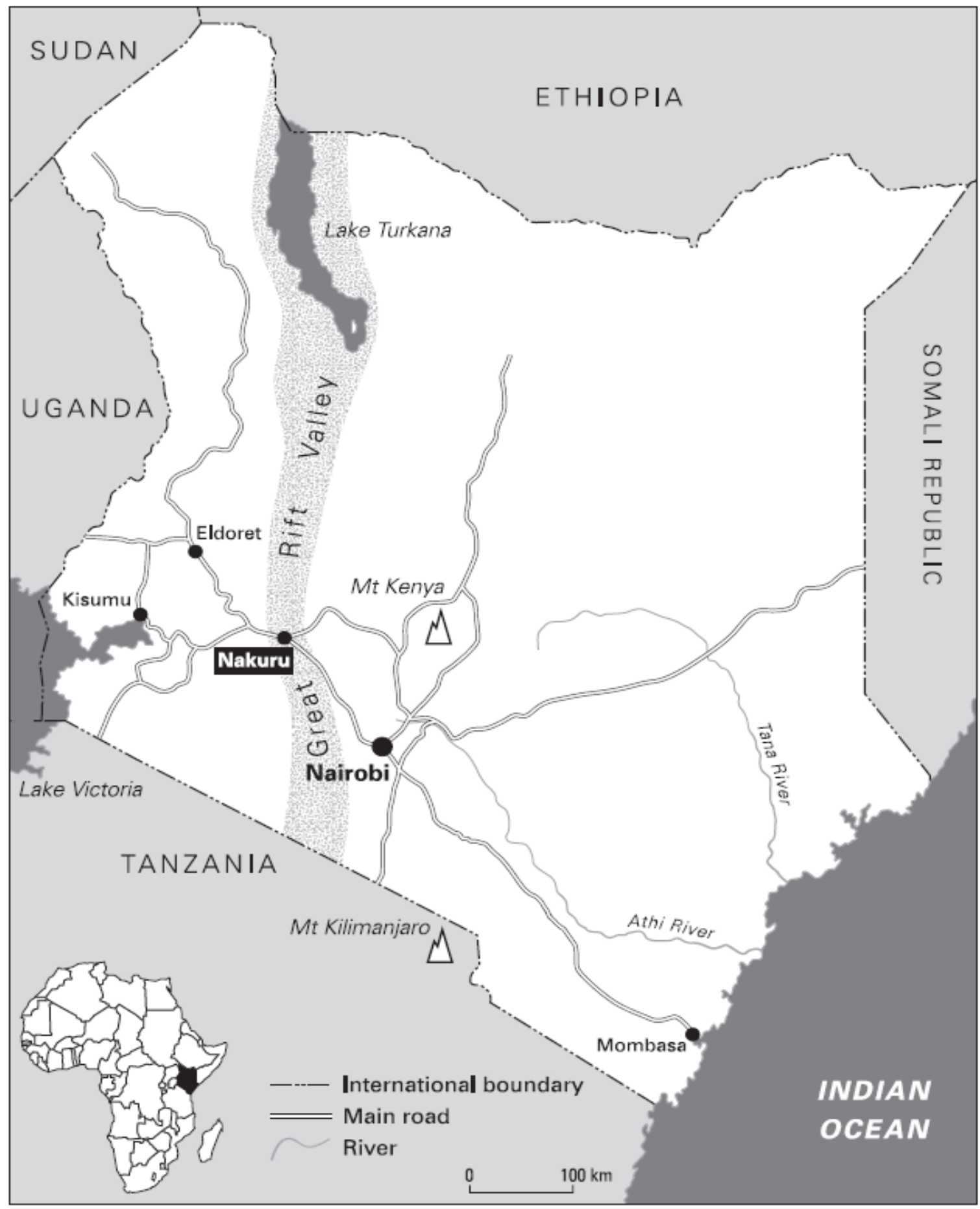

Adopted from Owuor (2006:38) 